\title{
Synchronization of spatiotemporal patterns and modeling disease spreading using excitable media
}

Jianxia Cui

West Virginia University

Follow this and additional works at: https://researchrepository.wvu.edu/etd

\section{Recommended Citation}

Cui, Jianxia, "Synchronization of spatiotemporal patterns and modeling disease spreading using excitable media" (2004). Graduate Theses, Dissertations, and Problem Reports. 2109.

https://researchrepository.wvu.edu/etd/2109

This Dissertation is protected by copyright and/or related rights. It has been brought to you by the The Research Repository @ WVU with permission from the rights-holder(s). You are free to use this Dissertation in any way that is permitted by the copyright and related rights legislation that applies to your use. For other uses you must obtain permission from the rights-holder(s) directly, unless additional rights are indicated by a Creative Commons license in the record and/ or on the work itself. This Dissertation has been accepted for inclusion in WVU Graduate Theses, Dissertations, and Problem Reports collection by an authorized administrator of The Research Repository @ WVU.

For more information, please contact researchrepository@mail.wvu.edu. 


\title{
Synchronization of Spatiotemporal Patterns and Modeling Disease Spreading Using Excitable Media
}

\author{
Jianxia Cui \\ DISSERTATION \\ Submitted to the Eberly College of Arts and Sciences of \\ West Virginia University \\ in Partial Fulfillment of the Requirements for \\ the Degree of
}

Doctor of Philosophy in Chemistry

\author{
Kenneth Showalter, Ph.D., Chairman \\ Charles Jaffé, Ph.D. \\ Terry Gullion, Ph.D. \\ Kung K. Wang, Ph.D. \\ Mark E. Koepke, Ph.D.
}

\begin{abstract}
Morgantown, West Virginia 2004
\end{abstract}

Keyword: Synchronization, Spatiotemporal Pattern, Modeling Disease Spreading, Excitable Media, Photosensitive BZ Reaction

Copyright 2004 Jianxia Cui 


\title{
ABSTRACT
}

\section{Synchronization of Spatiotemporal Patterns and Modeling Disease Spreading Using Excitable Media}

\author{
Jianxia Cui
}

Studies of the photosensitive Belousov-Zhabotinsky (BZ) reaction are reviewed and the essential features of excitable media are described. The synchronization of two distributed Belousov-Zhabotinsky systems is experimentally and theoretically investigated. Symmetric local coupling of the systems is made possible with the use of a video camera-projector scheme. The spatial disorder of the coupled systems, with random initial configurations of spirals, gradually decreases until a final state is attained, which corresponds to a synchronized state with a single spiral in each system. The experimental observations are compared with numerical simulations of two identical Oregonator models with symmetric local coupling, and a systematic study reveals generalized synchronization of spiral waves. Modeling studies on disease spreading have been reviewed. The excitable medium of the photosensitive BZ reaction is used to model disease spreading, with static networks, dynamic networks, and a domain model. The spatiotemporal dynamics of disease spreading in these complex networks with diffusive and non-diffusive connections is characterized. The experimental and numerical studies reveal that disease spreading in these model systems is highly dependent on the non-diffusive connections. 


\section{Dedication}

To my late father, the best father in the world

To my mother, the best mother in the world

To my husband, the best husband in the world without their love and support

this work would never have been finished. 


\section{Acknowledgements}

Having completed this work, I feel it is appropriate to emphasize how much I am indebted to all of the people who helped me during these years. First and foremost, I would like to express my gratitude to my research advisor and mentor, Dr. Kenneth Showalter, for his guidance, support, and help for my study at WVU. I learned from him not only the fasinating field of nonlinear dynamics but also about the "human side" of science.

I express my gratitude to Drs. Michael Hilderbrand, Eugene Mihaliuk, and Jichang Wang for their contributions and interest in the project Synchronization of Spatiotemporal Patterns in Locally Coupled Excitable Media and to Drs. Florin Chirila, Eugene Mihaliuk, Shi Zhong, and Mark Tinsley for their contributions and interest in the project Modeling Disease Spreading with Excitable Media. I also express my gratitude to Drs. Charles Jaffé, Terry Gullion, Mark E. Koepke, and Kung K. Wang for their directions. Special thanks go to Drs. Paul Jagodzinski and Harry Finklea, who helped make the Chemistry Department a comfortable place for students.

I am indebted to my friend Dr. Jichang Wang, who gave me much help in settling down for life and study in Morgantown. I am also indebted to my friend Dr. Renate Wackerbauer, from whom I learned a great deal about this research field and many things beyond it as well. Although Jichang and Renate have left Morgantown, I still receive their generous encouragement. I am grateful to Dr. Eugene Mihaliuk for his critical reading of the first four chapters of this dissertation, and for his kind help and constructive criticism from which I greatly benefited.

I wish to thank my collegues and friends who provided a friendly environment and shared my tears and laughs in these years. They are Sandor Kádár, Paul Kinkus, Jianan Feng, Tatsunari Sakurai, Hongyan Sun, Mitsuhiro Murata, Aaron Steele, Yongjun Li, Zhaoyang Hang, Tabitha Chigiwada, and Xiaojian Mao.

Finally, I am very grateful to my family, especially my late father and my mother, 
for their enormous support and understanding spiritually and emotionally concerning my education and professional pursuit over the years. To my sister and brothers, for their understanding that I could not take care of my father due to being in America. My deepest thanks go to my husband and soul mate, Feifei, for his willingness to forgo innumerable evenings and weekends and his endless love, care, support, and patience. 


\section{Contents}

Title Page $\quad$ i

Abstract

Dedication $\quad$ iii

Acknowledgements $\quad$ iv

Table of Contents vi vi

List of Tables $\quad \mathrm{x}$

List of Figures $\quad$ xi

Contents in Attached CD xvi

1 Introduction. The Light-Sensitive Belousov-Zhabotinsky Reaction 1

1.1 References . . . . . . . . . . . . . . . . . . 9

2 The Mechanism and Kinetic Model of the Belousov-Zhabotinsky $\begin{array}{ll}\text { Reaction } & 17\end{array}$ 
2.1 The FKN Mechanism . . . . . . . . . . . . . . . . . . . . . . 18

2.2 The Mechanism of Light Sensitivity in the $\mathrm{Ru}$ (bpy) ${ }_{3}{ }^{2+}$ Catalyzed BZ Reaction . . . . . . . . . . . 20

2.3 The Kinetic Model . . . . . . . . . . . . . . . . . . . . . . . . . . . . 23

2.4 Dimensionless Equations of the Oregonator Model . . . . . . . . . 26

2.5 References . . . . . . . . . . . . . . . . . . . . 31

3 Dynamic Media 33

3.1 Classification of Dynamic Media . . . . . . . . . . . . . . 33

3.2 Excitable Dynamics . . . . . . . . . . . . . . . . . . . . . . . . . 41

3.3 Excitable Media in the BZ Reaction . . . . . . . . . . . . . . . 51

3.4 References . . . . . . . . . . . . . . . . 56

4 Synchronization of Spatiotemporal Patterns in Locally Coupled Ex$\begin{array}{ll}\text { citable Media } & 60\end{array}$

4.1 Introduction . . . . . . . . . . . . . . 60

4.1.1 Category of schemes leading to synchronization _ . . . . . 61

4.1.2 Review of synchronization of spatiotemporal chaos . . . . . . 63

4.2 Experimental Study . . . . . . . . . . . . . . . . . . . . . . . 68

4.2 .1 Preparation of stock solutions . . . . . . . . . . . 68

4.2.2 Prepare of the catalyst-free BZ solution . . . . . . . . . 69

4.2 .3 Preparation of gel . . . . . . . . . . . . . . . . 70

4.2 .4 Experimental setup . . . . . . . . . . . . . 70

4.2 .5 Coupling scheme . . . . . . . . . . . . . . 76 
4.2.6 Experimental results I: Relaxation of disordered pattern to a synchronization state . . . . . . . . . . . . 80

4.2.7 Experimental results II: Spatiotemporal synchronization of spirals 83

4.3 Numerical Studies . . . . . . . . . . . . . . . . . . . . . . . 87

4.3.1 Numerical model . . . . . . . . . . . . . . . . . . . . . . . 87

4.3.2 Numerical simulations I: Relaxation of disordered pattern to a synchronization state . . . . . . . . . . . . 88

4.3.3 Numerical simulations II: Spatiotemporal synchronization of

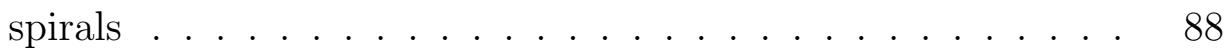

4.4 Discussion and Conclusions _. . . . . . . . . . . . . . . . 97

4.5 Appendix A: Synthesis of the Sulfato Salt of $\mathrm{Ru}(\mathrm{bpy})_{3}{ }^{2+} \ldots \ldots$

4.6 Appendix B: Main Part of the Program in OPTIMAS Used for the Experiments . . . . . . . . . . . . . . . . . . . 99

4.7 References . . . . . . . . . . . . . . . . . . 110

5 Modeling Disease Spreading with Excitable Media 120

5.1 Reviews of Modeling Disease Spreading . . . . . . . . . . . . . . 120

5.1.1 A brief history of modeling disease spreading . . . . . . . . . . 120

5.1 .2 Mathematical epidemiology models . . . . . . . . . . . . 121

5.1.3 Geographic spread of epidemics . . . . . . . . . . . . . 125

5.1.4 Disease spreading in networks . . . . . . . . . . . . 130

5.2 Modeling Disease Spreading with Excitable Media . . . . . . . . . . 138

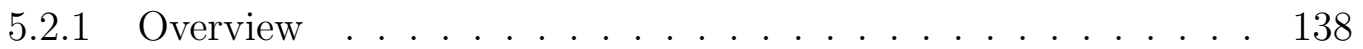

5.2 .2 Static networks . . . . . . . . . . . . . . . . . 142 
5.2 .3 Dynamic networks . . . . . . . . . . . . . . . . . . 157

5.2.4 Domain model networks . . . . . . . . . . . . . . . 176

$5.2 .5 \quad$ Discussion . . . . . . . . . . . . . . . . . . . . 181

5.3 Appendix A: The Program in OPTIMAS Used for Dynamic Networks in the Experiments . . . . . . . . . . . . . . . . . 186

5.4 Appendix B: The Program in OPTIMAS Used for Static Networks in the Experiments . . . . . . . . . . . . . . . . . . . 207

5.5 References . . . . . . . . . . . . . . . . . . . . . . 221 


\section{List of Tables}

2.1 The FKN mechanism . . . . . . . . . . . . . . . . . . . . . . . . 22

3.1 State variables of some representative excitable media . . . . . . . . 50

4.1 Characters of space-time chaos systems . . . . . . . . . . . . 64

4.2 Recipe of the catalyst-free BZ solution . . . . . . . . . . . . . . 69

4.3 Setting of the reference camera . . . . . . . . . . . 76

5.1 Population distribution in the domains . . . . . . . . . . . . 177 


\section{List of Figures}

3.1(a) Typical structure of $f(u, v) \ldots \ldots \ldots \ldots \ldots$

3.1(b) Typical structure of $g(u, v) \ldots \ldots \ldots \ldots \ldots \ldots$

3.1(c) The structure of $f(u, v)$ and the sections on 3 planes . . . . . 38

3.1(d) Contour lines of $f(u, v)$ and $g(u, v)$ in $u, v$ phase plane $\ldots \ldots .39$

3.2 The nullclines of an isolated active element . . . . . . . . 40

3.3(a) Typical phase plane for an excitable system, with $u$ and $v$ as the activator and inhibitor of the Oregonator model . . . . . . . . 42

3.3(b) Magnified region marked by dash-dotted square in Fig. 3.3(a) . . . 43

3.4(a) Trajectories at different initial values of $u$ and $v$ within the unit time $d t$ in the Oregonator model . . . . . . . . . . . . 45

3.4(b) The magnified trajectories converge along the right-hand branch of the $u$ nullcline in Fig. $3.4(\mathrm{a}) \ldots \ldots \ldots \ldots$

3.4(c) The magnified trajectories diverge along the middle branch of the $u$ nullcline in Fig. $3.4(\mathrm{a}) \ldots \ldots \ldots$. . . . . . . . . . 47

3.5 The effect of light intensity on the excitability of the photosensitive BZ reaction . . . . . . . . . . . . . . . . . . . . 5 53

3.6 Nullclines for the photosensitive BZ reaction $\ldots \ldots \ldots \ldots$

3.7 Another nullclines for the photosensitive BZ reaction . . . . . . 55 


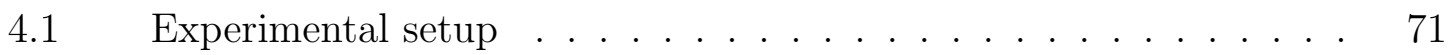

4.2 Observation angles and the effects $\ldots \ldots \ldots \ldots . \ldots 74$

4.3 The relationship between light intensity and the gray level . . . . 75

4.4 Typical example of an image and the mutual coupling image . . 78

$4.5 \quad$ Schematic diagram of coupling scheme . . . . . . . . . . . . 79

4.6 Experimental behavior of relaxation of an initially disordered array of spirals to a synchronized state in two locally coupled excitable media . . . . . . . . . . . . . . . . . . . 81

4.7 Experimental behavior of relaxation of an initially disordered array of spirals to a synchronized state in two locally coupled excitable media in the presence of colored noise . . . . . . . . . . . 82

4.8 $\quad$ BZ experiment with two single spirals rotating in opposite directions 84

4.9 BZ experiment with single spirals rotating in same direction with smaller initial distance . . . . . . . . . . . . . . . . . . 85

4.10 BZ experiment with single spirals rotating in same direction with larger initial distance . . . . . . . . . . . . . . . . 86

4.11 Numerical simulations of relaxation of an initially disordered array of spirals to a synchronized state in two locally coupled excitable media . . . . . . . . . . . . . . . . . . . . .

4.12 Results from numerical simulations for counter-rotating spirals . . 91

4.13 Results from numerical simulations for co-rotating spirals with larger initial distance . . . . . . . . . . . . . . . . . . . . . . . 9 92

4.14 Results from numerical simulations for co-rotating spirals with smaller initial distance . . . . . . . . . . . . . . . . . . . . . . 93 
4.15 Results from a numerical simulation of Eqs. (4.4) with clockwise

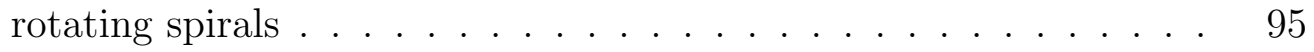

4.16 Results from a numerical simulation for the coupled Barkley system 96

5.1 "Small world" between a regular ring lattice and a random network 132

$5.2 \quad$ Scale-free graph . . . . . . . . . . . . . . . . 136

5.3(a) Static networks with 10 links . . . . . . . . . . . . . 144

5.3(b) Static networks with 100 links . . . . . . . . . . . 145

5.3 (c) Static networks with 500 links . . . . . . . . . . 146

5.4 Chemical wave in the static network system with the imposed longdistance interactions . . . . . . . . . . . . . . . . . 147

5.5(a) Experimental results for 100 static links . . . . . . . . . . . . 148

5.5(b) Experimental results for 300 links . . . . . . . . . . . . . 149

5.6(a) Time-series of fraction of infected elements in the experiment with 50 static links . . . . . . . . . . . . . . . . . 150

5.6(b) Power spectrum of the time-series of fraction of infected elements in the experiment with 50 static links . . . . . . . . . . . . 151

5.7(a) Time-series of fraction of infected elements in the experiment for 100 static links . . . . . . . . . . . . . . . . . 152

5.7(b) Power spectrum of the time-series of fraction of infected elements in the experiment for 100 static links . . . . . . . . . . . 153

5.8(a) Time-series of fraction of infected elements in the experiment for 300 static links . . . . . . . . . . . . . . . . . . 154

5.8(b) Power spectrum of the time-series of fraction of infected elements in the experiment for 300 static links . . . . . . . . . . . . 155 
5.9 Experimental results of normalized coverage time as a function of probability in static networks . . . . . . . . . . . . 156

5.10 Numerical simulations of time-series of fraction of infected elements 158

5.11 Simulation results of normalized first coverage time as a function of probability in static networks . . . . . . . . . . . . . . . 159

5.12 Experimental results in dynamic networks for $p=0.1 \ldots 161$

5.13 Experimental results in dynamic networks for $p=0.15 \ldots . . . . \quad 162$

5.14(a) Time-series of fraction of infected elements in the experiment for $p=0.1 \ldots \ldots \ldots \ldots \ldots \ldots$

5.14(b) Power spectrum of the time-series of fraction of infected elements in the experiment for $p=0.1 \ldots \ldots \ldots$. . . . . . . . . 164

5.15(a) Time-series of fraction of infected elements in the experiment for $p=0.15 \ldots \ldots \ldots \ldots \ldots \ldots$

5.15(b) Power spectrum of the time-series of fraction of infected elements in the experiment for $p=0.15 \ldots \ldots$. . . . . . . . . 166

5.16(a) Time-series of fraction of infected elements in the experiment for $p=0.18 \ldots \ldots \ldots \ldots \ldots \ldots$

5.16(b) Power spectrum of the time-series of fraction of infected elements in the experiment for $p=0.18 \ldots \ldots \ldots$. . . . . . . . . 168

5.17 Experimental results of normalized coverage time as a function of

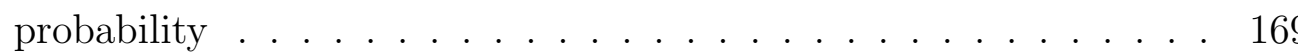

5.18 Numerical simulation results in dynamic networks for $p=0.0004 \quad 170$

5.19(a) Numerical simulations of time-series of fraction of infected elements with the probability of $0.00005 \ldots \ldots \ldots$. . . . . . . . 171

5.19(b) Power spectrum of time-series for $p=0.00005 \ldots$. . . . . . . . . . 172 
5.20(a) Numerical simulations of time-series of fraction of infected elements with the probability of $0.0004 \ldots \ldots \ldots$. . . . . . . . 173

5.20(b) Power spectrum of time-series for $p=0.0004 \ldots \ldots$. . . . . . . 174

5.21 Simulation results of normalized coverage time as a function of $p \quad$. 175

5.22 Zipf's law relationship between the size and the rank of the different sizes of the domains . . . . . . . . . . . . . . . . 178

5.23 Domains distribution used in the numerical simulations . . . . . 179

5.24 Numberical simulations of domain model networks for $p=0.00008 \quad 180$

5.25 Numberical simulations of domain model networks for $p=0.0008$. 182

5.26 Numerical simulations of time-series of fraction of infected elements in domain model . . . . . . . . . . . . . . . . . . 183 


\section{Contents in Attached CD}

1. pdf files of the dissertation $\ldots \ldots \ldots \ldots \ldots \ldots \ldots \ldots$ dissertation.pdf

2. Latex files of each chapter $\ldots \ldots \ldots \ldots \ldots \ldots \ldots \ldots \ldots$ folder "latex"

3. Figures in the dissertation $\ldots \ldots \ldots \ldots \ldots \ldots \ldots \ldots \ldots \ldots \ldots \ldots \ldots \ldots$ folder "figures"

4. Programs in OPTIMAS $\ldots \ldots \ldots \ldots \ldots \ldots \ldots \ldots \ldots \ldots$ folder "OPTIMAS"

4.1 Synchronization of spatiotemporal patterns $\ldots \ldots \ldots \ldots \ldots$ folder "syn"

4.2 Modeling disease spreading in static networks ........... folder "static"

4.3 Modeling disease spreading in dynamic networks ........ folder "dynamic"

4.4 Modeling disease spreading in domain model ........... folder "domain"

5. Programs in $\mathrm{C} \ldots \ldots \ldots \ldots \ldots \ldots \ldots \ldots \ldots \ldots \ldots \ldots \ldots \ldots \ldots \ldots \ldots \ldots \ldots$ folder "C-code"

5.1 Modeling disease spreading in static networks ........... folder "static"

5.2 Modeling disease spreading in dynamic networks ....... folder "dynamic"

5.3 Modeling disease spreading in domain model ............ folder "domain" 


\section{Chapter 1}

\section{Introduction. The Light-Sensitive Belousov-Zhabotinsky Reaction}

The Belousov-Zhabotinsky (BZ) reaction $[1,2]$ consists of the oxidation of malonic acid by bromate catalyzed by metal ion or metallo-complexes in acidic aqueous solution. The light-sensitive BZ reaction, in which a light-sensitive catalyst is employed, has attracted increasing attention in recent years. Numerous studies have been carried out in this field that can broadly be classified into four main topics: (a) the influence of light on temporal oscillations in well-stirred homogeneous systems under batch or continuous flow conditions [3-10]; (b) novel spatiotemporal patterns induced by light irradiation in a thin layer of solution or gel [11-24] (c) dynamics features of spatiotemporal patterns under the influence of illumination [25-54]; and (d) the chemical mechanism of the light-sensitivity [55-62] and kinetic model [63] allowing prediction of experimental observations by numerical simulations. In this Chapter, we briefly review representative studies of these four topics in chronological order.

The first studies on the effect of light on the temporal oscillations of the BZ reaction were in 1968, when Vavilin et al. [3] first reported that irradiation of ultraviolet (UV) light completely inhibited or strongly modified the oscillations in the cerium- 
catalyzed system. The currently widely used photosensitive BZ catalyst, tris $\left(2,2^{\prime}-\right.$ bipyridine)ruthenium(II), $\mathrm{Ru}(\mathrm{bpy})_{3}{ }^{2+}$, was first adapted as a luminescent indicator for the demonstration of the BZ oscillatory reaction by Demas and Diemente in 1973 [4]. Since then, $\gamma$-irradiation [6], visible light [7], and laser light [8] have been used to study light effects on the oscillations of the BZ reaction, catalyzed by either $\mathrm{Ce}^{3+}[6,7], \mathrm{Mn}^{2+}[6]$, iron-phenantroline (also known as ferroin or $\mathrm{Fe}(\mathrm{phen})_{3}{ }^{2+}$ ) [6-8], $\mathrm{Ru}(\mathrm{bpy})_{3}{ }^{2+}[6,7,9,10]$, potassium ferrioxalate $\left(\mathrm{K}_{3} \mathrm{Fe}\left(\mathrm{C}_{2} \mathrm{O}_{4}\right)\right)$ with dipyridyl [5], or ruthenium-dipyridile $\left(\mathrm{Ru}(\operatorname{dipy})_{3}^{2+}\right)[6]$.

The earlier studies were focused on the light effect on the oscillatory behavior, such as the shape, amplitude, frequency, initiation or inhibition of oscillations. Kuhnert and Linde [5] found in 1979 a proportional, differential, or proportional-differential response behavior in the potassium ferrioxalate $\left(\mathrm{K}_{3} \mathrm{Fe}\left(\mathrm{C}_{2} \mathrm{O}_{4}\right)\right)$ and dipyridyl catalyzed system followed the interrupting of a square-wave UV-light pulse. Körös et al. [6] found that $\gamma$-irradiation could quench or considerably decrease the frequency of oscillation if $\mathrm{Ce}^{4+}$ or $\mathrm{Mn}^{2+}$ was the catalyst; whereas it had no effect on the system catalyzed by metal complexes such as $\mathrm{Fe}(\text { phen })_{3}{ }^{2+}$ or $\mathrm{Ru}(\text { dipy })_{3}{ }^{2+}$. Gáspár et al. [7] in 1983 studied the influence of visible light on BZ oscillating systems using different catalysts in a batch reactor. They found that light has no effect on the $\mathrm{Ce}^{4+}$ system. However, the oscillating systems using the complexes $\mathrm{Fe}(\text { phen })_{3}{ }^{2+}$ or $\mathrm{Ru}(\mathrm{bpy})_{3}{ }^{2+}$ as catalyst were affected. Not only were the amplitude and frequency of oscillations changed by the illumination, but also the initiation and inhibition of oscillations were observed as a response to the illumination. In 1987, Bodet et al. [8] studied inhomogeneous perturbations of the BZ reaction catalyzed by ferroin with a focused laser beam and observed diffusion-phase waves in the oscillating medium. In 1992, Weigt [9] reported chemicluminescence oscillations in the $\mathrm{Ru}(\mathrm{bpy})_{3}{ }^{2+}$ catalyzed BZ reaction in a flow-through reactor, with three separate emission peaks. In 1993, Mori et al. [10] reported a bifurcation study in the $\mathrm{Ru}(\mathrm{bpy})_{3}{ }^{2+}$ catalyzed BZ system in a continuous stirred flow tank reactor (CSTR) as a function of bromate concentration and 
the light intensity.

As early as 1973, Busse and Hess [11] first demonstrated the initialization of a propagating wave by UV radiation in a thin layer of cerium-ferroin catalyzed BZ solution. Kuhnert [12] studied the transformation of phase waves into trigger waves by illuminating the $\mathrm{Ru}(\mathrm{bpy})_{3}{ }^{2+}$ catalyzed $\mathrm{BZ}$ reaction system. The exposure to light releases an inhibitor that stops the production of phase waves and converts them to trigger waves. Kuhnert [13] suggested a new kind of optical photochemical memory device based on his studies in 1986. Kuhnert and co-workers [14] also demonstrated image processing in this reaction system three years later.

During the 1990s, many more complex spatiotemporal patterns were observed in the $\mathrm{Ru}(\mathrm{bpy})_{3}{ }^{2+}$ catalyzed BZ reaction. Jinguji et al. [15] studied the photoinduced waves in square, triangular, star and circular shapes. Waves were initiated at the boundaries of the corresponding opaque masks and the wave fronts propagated inward into the illuminated field, contrary to the usual direction of wave motion. Müller and co-workers $[16,17]$ used an argon laser beam to produce an unexcitable disk in a BZ medium, which erased the core structure of two adjacent spirals, generating an autonomous pacemaker for target patterns [16] and constructing multi-armed spirals [17] with spiral tips at equal distances around the disk boundary. Wave splitting following a short, high-intensity inhibitory light pulse was observed by Krug et al. [18] and Muñuzuri et al. [19]. Petrov et al. [20] reported that a spiral wave transforms into a labyrinthine standing wave with periodic optical forcing and they found a sequence of frequency-locked resonant patterns as the forcing frequency was varied. Amemiya et al. [21,22] studied the formation and evolution of three-dimensional scroll waves in the $\mathrm{Ru}(\text { bpy })_{3}{ }^{2+}$ catalyzed $\mathrm{BZ}$ reaction by perturbing traveling waves transverse to their directions of prorogation. Vanag et al. [23] reported oscillatory cluster patterns generated by a global feedback. Hildebrand et al. [24] found complex behavior of colliding and splitting wave fragments with light-induced remote communication.

Trigger waves and spiral waves are characteristic of reaction-diffusion systems and 
they are observed in many biological and chemical media $[25,26]$. The photosensitive $\mathrm{BZ}$ reaction is the most widely studied chemical laboratory system for the dynamics of chemical waves, as the excitability of the medium can be efficiently controlled by either globally or locally altering the illumination. In the following, we present studies on the velocity of trigger waves and the tip motion of spiral waves.

The velocity of a trigger wave is a characteristic property of a reaction-diffusion system. Its systematic study provides a key to understanding the properties of the system. Kuhnert and Krug [27] first quantitatively studied the wave propagation in the non-illuminated $\mathrm{Ru}(\mathrm{bpy})_{3}{ }^{2+}$ catalyzed systems and compared the results with ferroin system [64]. Reddy et al. [28] systematically studied the influence of visible light on wave velocities in the $\mathrm{Ru}(\mathrm{bpy})_{3}{ }^{2+}$ catalyzed $\mathrm{BZ}$ reaction in a thin film of solution. Light was found to decrease the velocity and even completely inhibit the wave propagation for the concentration conditions studied. The velocity of wave propagation increases with increasing concentration of $\mathrm{Ru}(\mathrm{bpy})_{3}{ }^{2+}$ and is proportional to the square root of the product of the concentrations of $\mathrm{H}_{2} \mathrm{SO}_{4}$ and $\mathrm{NaBrO}_{3}$, which was first shown by Field and Noyes for the ferroin catalyzed system [64]. Meanwhile, Krug et al. [18] studied the tris(4,4'-dimethyl-2,2'-bipyridyl)ruthenium(II) $\left(\mathrm{Ru}(\mathrm{dmpy})_{3}{ }^{2+}\right)$ catalyzed BZ system in a continuously fed gel reactor and also found that trigger wave velocity decreased with increasing light intensity until the waves were completely extinguished.

The excitability of the medium affects not only the velocity of the wave but also the other characteristics of wave propagation. Sendiña-Nadal et al. [29] studied wave propagation in a medium with disordered excitability. They found that wave speed in one dimension was smaller than that corresponding to a homogenous medium, while in two dimensions, wave velocity increased due to the roughening of the front. Agladze et al. [30] studied the propagation of chemical waves in a photosensitive BZ system at the boundary of excitable and inhibitory fields. Depending on the degree of excitability of the two areas, waves were found to either penetrate into the inhibitory 
region or collapse in the excitable zone. Kádár et al. [31] reported stochastic resonance in a subexcitable medium, where wave propagation was significantly improved by spatiotemporal noise in a medium unable to support sustained wave propagation. Noise driven avalanche behavior was also studied [32] and sustained wave propagation by periodic modulation of a homogeneous illumination was studied [33]. A negativefeedback control algorithm was used to stabilize the propagating wave segments [34]. Recently, wave propagation in intricate patterns controlled by feedback-regulated excitability gradients in excitable media has been reported [35], which may one day lead to controlling abnormal electrical waves in the heart or brain to ward off a heart attack or epileptic seizure.

Markus et al. [36] studied in 1992 the phototaxis of spiral waves, which drift toward regions of higher light intensity. Steinbock and Müller [37] found the rotation period, wavelength and velocity of spirals increased monotonically when an argon laser beam radius was increased, which served as the core of the spiral. Agladze et al. [38] found that the spiral wave could completely disappear under a rapid increase of illumination or survive and reshape its core under a slow increase of illumination; they found multiple wave breaks at the periphery of the spiral wave when the illumination changed at a moderate speed. Petrov et al. [39] examined the behavior of spirals for a wide range of bromate concentrations and presented the primary bifurcations in frequency and wavelength of the spirals as a function of malonic acid and bromate concentrations and the illumination intensity in the $\mathrm{Ru}(\mathrm{bpy})_{3}{ }^{2+}$ catalyzed BZ reaction. They used a continuous fed unstirred reactor (CFUR).

The trajectory of the tip of a spiral wave, which is an important characteristic of the spiral wave motion, is very sensitive to changes of external illumination. Braune and Engel [40] investigated the compound rotation of the tip of spiral waves under constant illumination. They found the tip motion transformed from nearly rigid rotation into various meandering regimes and again into rigid rotation when the light intensity gradually increased. Brandtstädter et al. [41] studied the tip trajectory as 
well by using $\mathrm{Ru}(\mathrm{dmbpy})_{3}{ }^{2+}$ instead of $\mathrm{Ru}(\mathrm{bpy})_{3}{ }^{2+}$ as the catalyst. The tip trajectory undergoes a compound rotation to form an outward hypocycloid and an inward epicycloid-like trajectory as the light intensity increases. If a spiral wave is subjected to spatiotemporal structured noise, the tip trajectory exhibits a Brownian motion, as reported by Sendiña-Nadal et al. [42]. All of the above studies have used an excitable medium. The spiral wave in an oscillatory medium has also been studied [43]. It was found that irradiation with visible light in the core region of the vortex in an oscillatory medium results in a bifurcation of the vortex shape from an Archimedian spiral wave to a phase rotor.

Under time-dependent harmonic modulation of the uniform illumination, a synchronization of the movement of the spiral tip with the external frequency within entrainment bands, a resonance drift of the spiral core, and irregular motion of the tip have been observed by several authors [44-47]. If the spiral wave was disturbed by a sequence of short light pulses at a particular detection point, two new dynamic regimes for spiral tip behavior, named entrainment and resonance attractors, were found and studied in detail [48-51]. If the intensity of the time-dependent uniform modulation is changed proportional to the average wave activity in a confined circular domain, the stabilization and destabilization of the rigid rotation tip of a spiral wave will occur according to the control parameter [52].

The dynamics of a pair of spiral waves has also been studied. Agladze [53] studied the light-induced collapse of a pair of counter-rotating spiral waves in an active medium based on the $\mathrm{Ru}(\mathrm{bpy})_{3}{ }^{2+}$ catalyzed BZ reaction. Spiral waves annihilate only if the light intensity is increased in proper phase relative to the rotation of the spiral waves, otherwise the distance between spiral wave cores increases and the pair survives. Brandtstädter et al. [41] used $\mathrm{Ru}(\mathrm{dmbpy})_{3}{ }^{2+}$ as a catalyst to study the interaction of spiral pairs in an open-gel reactor. They found a small, initially symmetric pair of counter-rotating spiral waves underwent a symmetry-breaking instability after several rotation periods. They studied a pseudo-spiral pair by constructing a 
plane boundary impermeable to diffusion and they measured the spiral drift along the boundary as a function of the applied light intensity in repulsive and attractive interactions.

All of the above studies are carried out on the ruthenium(II) catalyzed BZ reaction. Guderian et al. [54] demonstrated resonant chaos controlled by light in the chemiluminescent BZ reaction, which is catalyzed by a mixture of $\mathrm{Ce}_{2}\left(\mathrm{SO}_{4}\right)_{3}$ and $\mathrm{Ru}(\text { bpy })_{3}{ }^{2+}$. Tóth et al. [65] recently reported wave initiated in the ferroin-catalyzed BZ reaction on a polysulfone membrane by visible light of $632.8 \mathrm{~nm}$ wavelength from a He-Ne laser. Their observations are in contrast to the inhibitory effect of visible light in the $\mathrm{Ru}(\text { bpy })_{3}{ }^{2+}$ catalyzed $\mathrm{BZ}$ system.

Among the light-sensitive $\mathrm{BZ}$ reactions, the $\mathrm{Ru}(\mathrm{bpy})_{3}{ }^{2+}$ catalyzed reactions have received much attention due to the specific photosensitive properties of the $\mathrm{Ru}(\mathrm{bpy})_{3}{ }^{2+}$ complex [66]. Light at $452 \mathrm{~nm}$ produces an excited state of the ruthenium catalyst, $\mathrm{Ru}$ (bpy) ${ }_{3}{ }^{2+*}$, which is an extremely strong reducing agent. Both inhibition [6] and induction [10] of oscillation caused by light has been reported, and wave initiation [15], induction [18,19], inhibition [28] and elimination $[18,28,38]$ has been demonstrated. We can summarize that light acts both as an inhibitor and as an accelerator in this system. It is well known that in the $\mathrm{BZ}$ reaction bromide $\left(\mathrm{Br}^{-}\right)$is the inhibitor and bromous acid $\left(\mathrm{HBrO}_{2}\right)$ is the autocatalyst [67]. Studies on the mechanism of the photosensitive BZ reaction have revealed how these two intermediates are generated in this complex system. Most studies are focused on the $\mathrm{Br}^{-}$generation by monitoring its concentration using a bromide ion selective electrode. Some authors found that bromide was produced by an excited state of $\mathrm{Ru}(\mathrm{bpy}){ }_{3}{ }^{2+}$ in its reaction with bromomalonic acid (BrMA) [59,60,62], which is used very commonly to explain studies of spatiotemporal pattern. Although bromide was thought to be a product of the reaction of the excited state of $\mathrm{Ru}$ (bpy $)_{3}{ }^{2+}$ with bromate $[13,56]$, other studies excluded this mechanism $[57,60,62]$. It has also been found that the excited state of $\mathrm{Ru}(\mathrm{bpy})_{3}{ }^{2+}$ reacts with $\mathrm{HBrO}_{2}$ to generate $\mathrm{Br}^{-}$[55]. As for the generation of 
$\mathrm{HBrO}_{2}$, there are not so many arguments. Bromate reacting with the excited state of $\mathrm{Ru}$ (bpy) $)_{3}{ }^{2+}$ in the absence of BrMA to produce $\mathrm{HBrO}_{2}$ has been reported [56, 60-62]. Although quenching methods revealed that the amount of $\mathrm{HBrO}_{2}$ produced is much more than that of $\mathrm{Br}^{-}$from the photochemical reaction [58], these results conflict with more recent studies. Kádár et al. [60] have found that light affects the $\mathrm{Ru}(\mathrm{bpy})_{3}{ }^{2+}$ catalyzed BZ reaction via two separate photochemical pathways. The predominant effect is the production of $\mathrm{Br}^{-}$from the reduction of bromomalonic acid by the photoexcited catalyst. A secondary effect is the generation of $\mathrm{HBrO}_{2}$ from the reduction of $\mathrm{BrO}_{3}^{-}$by the excited catalyst. The production of $\mathrm{Br}^{-}$has been adopted in the kinetic model [63] which has qualitatively described a variety of dynamical behaviors observed in the photosensitive BZ system [24,30-35,41,42,47]. We will present the mechanism and the kinetic model in detail in the next Chapter. 


\subsection{References}

[1] B. P. Belousov, "Oscillation Reaction and its Mechanism (in Russian)," Sbornik refceratov po Radiacioni Medicine, 145, 1958 meeting (1959).

[2] A. M. Zhabotinsky, "Periodic Liquid-Phase Oxidation Reaction," Dokl. Akad. Nauk SSR 157, 392-395 (1964).

[3] V. A. Vavilin, A. M. Zhabotinsky, and A. N. Zaikin, "Effect of Ultraviolet Radiation on the Self-Oscillatory Oxidation of Malonic Acid Derivatives," Russ. J. Phys. Chem. 42, 3091-3094 (1968).

[4] J. N. Demas and D. J. Diemente, "An Oscillating Chemical Reaction with a Luminescent Indicator," J. Chem. Ed. 50, 357-358 (1973).

[5] L. Kuhnert and H. Linde, "Signal-Antwort-Verhalten von Autokatalytischen Reaktionssystemen," Z. Phys. Chemie. (Leipzig) 260, 423-434 (1979).

[6] E. Körös, M. Varga, and G. Putirskaya, "Perturbation of Bromate Oscillators," in Nonlinear Phenomena in Chemical Dynamics, edited by C. Vidal and A. Pacault (Springer, Berlin, 1981) p. 207-212.

[7] V. Gáspár, G. Bazas, and M. T. Beck, "The Influence of Visible Light in the Belousov-Zhabotinsky Oscillating Reactions Applying Different Catalysts," Z. Phys. Chemie. (Leipzig) 264, 43-48 (1983).

[8] J. M. Bodet, J. Ross, and C. Vidal, "Experiments on Phase Diffusion Waves," J. Chem. Phys. 86, 4418-4424 (1987).

[9] H. R. Weight, "Chemiluminescence Oscillations Driven by a Flow-Through Reactor in the $\mathrm{Ru}(\mathrm{bpy})_{3}{ }^{2+}$ Catalyzed BZ Reaction," Angew. Chem. Int. Ed. Engl. 31, 355-357 (1992). 
[10] Y. Mori, Y. Nakamichi, T. Sekiguchi, N. Okazaki, T. Matsumura and I. Hanazaki, "Photoinduction of Chemical Oscillation in the Belousov-Zhabotinsky Reaction under the Flow Condition," Chem. Phys. Lett. 211, 421-424 (1993).

[11] H. Busse and B. Hess, "Information Transmission in a Diffusion-Coupled Oscillatory Chemical System," Nature 244, 203-205 (1973).

[12] L. Kuhnert, "Photochemische Manipulation von Chemischen Wellen," Naturwiss. 73, 96-97 (1986).

[13] L. Kuhnert, "A New Optical Photochemical Memory Device in a Light-Sensitive Chemical Active Medium," Nature 319, 393-394 (1986).

[14] L. Kuhert, K. I. Agladze, and V. I. Krinsky, "Image Processing Using LightSensitive Chemical Waves," Nature 337, 244-247 (1989).

[15] M. Jinguji, M. Ishihara, and T. Nakazawa, "Photo-Induced Formation of Spatial Patterns in the Belousov-Zhabotinsky Reaction," J. Phys. Chem. 94, 1226-1229 (1990).

[16] S. Müller, O. Steinbock, and J. Schütze, "Autonomous Pacemaker of Chemical Waves Created by Spiral Annihilation," Physica A 188, 47-54 (1992).

[17] O. Steinbock and S. Müller, "Multi-Armed Spirals in a Light-Controlled Excitable Reaction," Int. J. of Bifurcat. Chaos 3, 437-443 (1993).

[18] H.-J. Krug, H. Brandtstädter, and L. Pohlmann, "Nucleation and Wave Propagation in the Modified Oregonator and Comparison with Experiments in a Photosensitive Belousov-Zhabotinsky Gel Reactor," J. Phys. Chem. 99, 10237-10245 (1995).

[19] A. P. Muñuzuri, V. Pérez-Villar, and M. Markus, "Splitting of Autowaves in an Active Medium," Phys. Rev. Lett. 79, 1941-1944 (1997). 
[20] V. Petrov, Q. Ouyang, and H. L. Swinney, "Resonant Pattern Formation in a Chemical System," Nature 388, 655-657 (1997).

[21] T. Amemiya, S. Kádár, P. Kettunen, and K. Showalter, "Spiral Wave Formation in Three-Dimensional Excitable Media," Phys. Rev. Lett. 77, 3244-3247 (1996).

[22] T. Amemiya, P. Kettunen, S. Kádár, T. Yamaguchi, and K. Showalter, "Formation and Evolution of Scroll Waves in Photosensitive Excitable Media," Chaos 8, 872-878 (1998).

[23] V. K. Vanag, L. Yang, M. Dolnik, A. M. Zhabotinsky, and I. R. Epstein, "Oscillatory Cluster Patterns in a Homogeneous Chemical System with Global Feedback," Nature 406, 389-391 (2000).

[24] M. Hildebrand, H. Skødt, and K. Showalter, "Spatial Symmetry Breaking in the Belousov-Zhabotinsky Reaction with Light-Induced Remote Communication," Phys. Rev. Lett. 87, 088303(1-4) (2001).

[25] R. Kapral and K. Showalter, Eds, Chemical Waves and Patterns (Kluwer, Dordrecht, 1995).

[26] I. R. Epstein and K. Showalter, "Nonlinear Chemical Dynamics: Oscillations, Patterns, and Chaos," J. Phys. Chem. 100, 13132-13147 (1996).

[27] L. Kuhnert and H.-J. Krug, "Kinetics of Chemical Waves in the Acidic BromateMalonic Acid-Ru(bpy) ${ }_{3}{ }^{2+}$ System in Comparison with the Ferroin System," J. Phys. Chem. 91, 730-733 (1987).

[28] M. K. Ram Reddy, Zs. Nagy-Ungvarai, and S. C. Müller, "Effect of Visible Light on Wave Propagation in the Ruthenium-Catalyzed Belousov-Zhabotinsky Reaction," J. Phys. Chem. 98, 12255-12259 (1994). 
[29] I. Sendiña-Nadal, A. P. Muñuzuri, D. Vives, V. Pérez-Munuzuri, J. Casademunt, L. Ramírez-Piscina, J. M. Sancho, and F. Sagués, "Wave Propagation in a Medium with Disordered Excitability," Phys. Rev. Lett. 80, 5437-5440 (1998).

[30] K. Agladze, Á. Tóth, T. Ichino, and K. Yoshikawa, "Propagation of Chemical Waves at the Boundary of Excitable and Inhibitory Fields," J. Phys. Chem. A. 104, 6677-6680 (2000).

[31] S. Kádár, J. Wang, and K. Showalter, "Noise-Supported Travelling Waves in Sub-Excitable Media," Nature 391, 770-772 (1998).

[32] J. Wang, S. Kádár, P. Jung, and K. Showalter, "Noise Driven Avalanche Behavior in Subexcitable Media," Phys. Rev. Lett. 82, 855-858 (1999).

[33] I. Sendiña-Nadal, E. Mihaliuk, J. Wang, V. Pérez-Munuzuri, and K. Showalter, "Wave Propagation in Subexcitable Media with Periodically Modulated Excitability," Phys. Rev. Lett. 86, 1646-1649 (2001).

[34] E. Mihaliuk, T. Sakurai, F. Chirila, and K. Showalter, "Experimental and Theoretical Studies of Feedback Stabilization of Propagating Wave Segments," Faraday Discuss. Chem. Soc. 120, 383-394 (2002).

[35] T. Sakurai, E. Mihaliuk, F. Chirila, and K. Showalter, "Design and Control of Wave Propagation Patterns in Excitable Media," Science 296, 2009-2012 (2002).

[36] M. Markus, Z. Nagy-Ungvarai, and B. Hess, "Phototaxis of Spiral Waves," Science 257, 225-227 (1992).

[37] O. Steinbock and S. C. Müller, "Chemical Spiral Rotation Is Controlled by LightInduced Artificial Cores," Physica A 188, 61-67 (1992).

[38] K. Agladze, V. Voignier, E. Hamm, F. Plaza, and V. Krinsky, "Fast Selective Elimination of Spiral Waves," J. Phys. Chem. 100, 18764-18769 (1996). 
[39] V. Petrov, Q. Ouyang, G. Li, and H. L. Swinney, "Light-Induced Frequency Shift in Chemical Spirals," J. Phys. Chem. 100, 18992-18996 (1996).

[40] M. Braune and H. Engel, "Compound Rotation of Spiral Waves in a LightSensitive Belousov-Zhabotinsky Medium," Chem. Phys. Lett. 204, 257-264 (1993).

[41] H. Brandtstädter, M. Braune, I. Schebesch, and H. Engel, "Experimental Study of the Dynamics of Spiral Pairs in Light-Sensitive Belousov-Zhabotinsky Media Using an Open-Gel Reactor," Chem. Phys. Lett. 323, 145-154 (2000).

[42] I. Sendiña-Nadal, S. Alonso, A. Pérez-Muñuzuri, M. Gómez-Gesteira, A. PérezVillar, L. Ramírez-Piscina, J. Casademunt, J. M. Sancho, and F. Sagués, "Brownian Motion of Spiral Waves Driven by Spatiotemporal Structures Noise," Phys. Rev. Lett. 84, 2734-2737 (2000).

[43] R. R. Aliev, T. Amemiya, and T. Yamaguchi, "Bifurcation of Vortices in the Light-Sensitive Oscillatory Belousov-Zhabotinsky Medium," Chem. Phys. Lett. 257, 552-556 (1996).

[44] M. Braune and H. Engel, "Compound Rotation of Spiral Waves in Active Media with Periodically Modulated Excitability," Chem. Phys. Lett. 211, 534-540 (1993).

[45] M. Braune, A. Schrader, and H. Engel, "Entrainment and Resonance of Spiral Waves in Active Media with Periodically Modulated Excitability," Chem. Phys. Lett. 222, 358-362 (1994).

[46] O. Steinbock, V. Zykov and S. C. Müller, "Control of Spiral-Wave Dynamics in Active Media by Periodic Modulation of Excitability," Nature 366, 322-324 (1993). 
[47] V. Zykov, O. Steinbock, and S. C. Müller, "External Forcing of Spiral Waves," Chaos 4, 509-518 (1994).

[48] S. Grill, V. S. Zykov, and S. C. Müller, "Feedback-Controlled Dynamics of Meandering Spiral Waves," Phys. Rev. Lett. 75, 3368-3371 (1995).

[49] S. Grill, V. S. Zykov, and S. C. Müller, "Spiral Wave Dynamics under Pulsatory Modulation of Excitability," J. Phys. Chem. 100, 19082-19088 (1996).

[50] D. M. Goldschmidt, V. S. Zykov, and S. C. Müller, "Transition to Irregular Dynamics of Spiral Waves under Two-Channel Feedback," Phys. Rev. Lett. 80, 5220-5223 (1998).

[51] O.-U. Kheowan, V. S. Zykov, O. Rangsiman, and S. C. Müller, "Transitions between Orbits of Resonance Attractors for Spiral Waves," Phys. Rev. Lett. 86, 2170-2173 (2001).

[52] O.-U. Kheowan, C.-K. Chan, V. S. Zykov, O. Rangsiman, and S. C. Müller, "Spiral Wave Dynamics under Feedback Derived from a Confined Circular Domain," Phys. Rev. E. 64, 035201(1-4) (2001).

[53] K. Agladze, "Light Induced Annihilation and Shift of Spiral Waves," Chaos 6, 328-333 (1996).

[54] A. Guderian, A. F. Münster, M. Jinguji, M. Kraus, and F. W. Schneider, "Resonant Chaos Control by Light in a Chemiluminescent Reaction," Chem. Phys. Lett, 312, 440-446 (1999).

[55] P. K. Srivastava, Y. Mori and I. Hanazaki, "Photo-Inhibition of Chemical Oscillation in the $\mathrm{Ru}(\mathrm{bpy})_{3}{ }^{2+}$-Catalyzed Belousov-Zhabotinsky Reaction," Chem. Phys. Lett. 190, 279-284 (1992). 
[56] M. Jinguji, M. Ishihara, and T. Nakazawa, "Primary Process of Illumination Effect on the $\mathrm{Ru}(\mathrm{bpy})_{3}{ }^{2+}$-Catalyzed Belousov-Zhabotinsky Reaction," J. Phys. Chem. 96, 4279-4281 (1992).

[57] M. K. R. Reddy, Z. Szlávik, Zs. Nagy-Ungvarai, and S. C. Müller, "Influence of Light on the Inorganic Part of the Ruthenium-Catalyzed Belousov-Zhabotinsky Reaction,” J. Phys. Chem. 99, 15081-15085 (1995).

[58] P. G. Sørensen, T. Lorenzen, and F. Hynne, "Quenching of Chemical Oscillations with Light," J. Phys. Chem. 100, 19192-19196 (1996).

[59] T. Yamaguchi, Y. Shimammoto, T. Amemiya, M. Yoshimoto, T. Ohmori, M. Nakaiwa, T. Akiya, M. Sato, and T. Natsumura-Inoue, "Bromomalonic Acid as a Source of Photochemically Produced $\mathrm{Br}^{-}$Ion in the $\mathrm{Ru}(\text { bpy })_{3}{ }^{2+}$-Catalyzed Belousov-Zhabotinsky Reaction," Chem. Phys. Lett. 259, 219-224 (1996).

[60] S. Kádár, T. Amemiya, and K. Showalter, "Reaction Mechanism for Light Sensitivity of the $\mathrm{Ru}$ (bpy) ${ }_{3}{ }^{2+}$-Catalyzed Belousov-Zhabotinsky Reaction," J. Phys. Chem. A 101, 8200-8206 (1997).

[61] A. Kaminaga, Y. Mori, and I. Hanazaki, "Photo-Induced Excitability in the tris-(bipyridyl)Ru(II)-Catalyzed Belousov-Zhabotinsky Reaction," Chem. Phys. Lett. 279, 339-343 (1997).

[62] L. Treindl, D. Knudsen, T. Nakamura, T. Matsumura-Inoue, K. B. Jørgenson and P. Ruoff, "The Light-Perturbed Ru-Catalyzed Belousov-Zhabotinsky Reaction: Evidence for Photochemically Produced Bromous Acid and Bromide Ions by Phase Response Analysis," J. Phys. Chem. A 104, 10783-10788 (2000).

[63] H.-J. Krug, L. Pohlmann, and L. Kuhnert, "Analysis of the Modified Complete Oregonator Accounting for Oxygen Sensitivity and Photosensitivity of BelousovZhabotinsky Systems," J. Phys. Chem. 94, 4862-4866 (1990). 
[64] R. J. Field and R. M. Noyes, "Oscillations in Chemical Systems. V. Quantitative Explanation of Band Migration in the Belousov-Zhabotinsky Reaction," J. Am. Chem. Soc. 96, 2001-2006 (1974).

[65] R. Tóth, V. Gáspár, A. Belmonte, M. C. O'Connel, A. Taylor, and S. K. Scott, "Wave Initiation in the Ferroin-Catalysed Belousov-Zhabotinsky Reaction with Visible Light," Phys. Chem. Chem. Phys. 2, 413-416 (2000).

[66] K. Kalyanasundaram, "Photophysics, Photochemistry and Solar Energy Conversion with Tris(bipyrirdyl)Ruthenium(II) and Its Analogues," Coord. Chem. Rev. 46, 159-244 (1982).

[67] R. J. Field, and M. Burger, Eds, Oscillations and Travelling Waves in Chemical Systems (Wiley, New York, 1985). 


\section{Chapter 2}

\section{The Mechanism and Kinetic Model of the Belousov-Zhabotinsky Reaction}

The widely studied Belousov-Zhabotinsky (BZ) [1,2] reaction was discovered by B. P. Belousov in 1951 and was extensively studied by A. M. Zhabotinsky as early as 1961 [3]. Belousov observed oscillations of the solution color during the cerium-catalyzed oxidation of citric acid by bromate. Later, Zhabotinsky replaced the original citric acid with malonic acid to improve the optical contrast of the color oscillations [3]. In a stirred sulfuric acid solution, initially containing potassium bromate, cerium sulfate, and malonic acid, the concentrations of bromide ion and cerium(IV) undergo repeated oscillations of large amplitude, with the reaction mixture changing from colorless to yellow and back at regular intervals. In 1972, Field, Körös and Noyes [4] presented a detailed chemical mechanism of the BZ reaction involving ten elementary steps, which became known as the FKN mechanism. In 1974, Field and Noyes [5] proposed a distillation of the FKN mechanism, a three variable scheme known as the Oregonator. Since then, the BZ reaction has become one of the most widely studied chemical reactions and is extensively used as a model for non-equilibrium systems $[6,7]$.

In this chapter, we present the FKN mechanism [4], the mechanism of the pho- 
tosensitive ruthenium(II) catalyzed BZ reaction proposed by Kádár et al. [8], the Oregonator [5] scaled by Tyson and Fife [9] for modeling temporal oscillations and spatiotemporal behaviors of the BZ reaction, and the modified complete Oregonator (MCO) for photosensitive BZ reaction presented by Kruhernt et al. [10]. Finally, we present the kinetic model used in the numerical simulations in this dissertation.

\subsection{The FKN Mechanism}

The primary overall reaction of the $\mathrm{BZ}$ reaction is,

$$
2 \mathrm{BrO}_{3}^{-}+3 \mathrm{CH}_{2}(\mathrm{COOH})_{2}+2 \mathrm{H}^{+} \rightarrow 2 \mathrm{BrCH}(\mathrm{COOH})_{2}+3 \mathrm{CO}_{2}+4 \mathrm{H}_{2} \mathrm{O} .
$$

Field, Körös and Noyes elucidated the detailed chemical mechanism in 1972. Table 2.1 shows the elementary reactions of the detailed mechanism numbered as in the original manuscript. Three processes were proposed as essential components of the reaction: Process A, the reduction of $\mathrm{BrO}_{3}^{-}$to $\mathrm{Br}_{2}$ by a series of oxygen atom transfers, and the subsequent bromination of malonic acid by $\mathrm{Br}_{2}$; Process $\mathrm{B}$, the reduction of $\mathrm{BrO}_{3}^{-}$by the autocatalytic species $\mathrm{HBrO}_{2}$, involving one-electron transfers among free radical oxybrominine intermediates in which the electron is supplied by $\mathrm{Ce}^{3+}$. The $\mathrm{Br}_{2}$ generated combines with malonic acid as in process $\mathrm{A}$. Process $\mathrm{C}$ is the oxidation

of organic and bromo-organic compounds by $\mathrm{Ce}^{4+}$ to regenerate $\mathrm{Ce}^{3+}$ and $\mathrm{Br}^{-}$. The three processes take place successively to constitute one oscillation, and the sequence is then repeated to generate successive oscillations. When $\mathrm{Br}^{-}$is consumed to a critical concentration in process $\mathrm{A}$, the autocatalysis in process $\mathrm{B}$ takes place. The $\mathrm{Br}^{-}$regeneration in process $\mathrm{C}$ effectively "resets the clock" by returning the system to process A.

In process A, when the solution contains a sufficient concentration of bromide ion $\left(\mathrm{Br}^{-}\right), \mathrm{BrO}_{3}^{-}$is reduced to $\mathrm{Br}_{2}$ by successive oxygen atom transfers (the two-equivalent redox processes (R1) and (R2)), and the malonic acid is brominated by an enolization 
mechanism, (R8):

$$
2 \mathrm{Br}^{-}+\mathrm{BrO}_{3}^{-}+3 \mathrm{CH}_{2}(\mathrm{COOH})_{2}+3 \mathrm{H}^{+} \rightarrow 3 \mathrm{BrCH}(\mathrm{COOH})_{2}+3 \mathrm{H}_{2} \mathrm{O} \text {. }
$$

When the concentration of bromide ion becomes very low and consequently the rate of its reaction with $\mathrm{BrO}_{3}^{-}$in (R3) becomes very small, an alternative pathway for the reaction of $\mathrm{BrO}_{3}^{-}$with $\mathrm{HBrO}_{2}$, (R5), becomes dominant. The $\mathrm{BrO}_{2}$. radical is generated, which oxidizes cerium(III) by the one-equivalent redox process (R6). The net reaction (2.3) represents an autocatalytic production of $\mathrm{HBrO}_{2}$ :

$$
\mathrm{HBrO}_{2}+\mathrm{BrO}_{3}^{-}+2 \mathrm{Ce}^{3+}+3 \mathrm{H}^{+} \rightleftharpoons 2 \mathrm{Ce}^{4+}+2 \mathrm{HBrO}_{2}+\mathrm{H}_{2} \mathrm{O} \text {. }
$$

An indefinite buildup of $\mathrm{HBrO}_{2}$ concentration is prevented by its second-order disproportionation in (R4):

$$
2 \mathrm{HBrO}_{2} \rightarrow \mathrm{HOBr}+\mathrm{BrO}_{3}^{-}+\mathrm{H}^{+} .
$$

Combining reaction (2.3) with reactions (R1), (R4), and (R8), (i.e., $2(2.3)+(\mathrm{R} 1)+(\mathrm{R} 4)$ $+(\mathrm{R} 8))$, we obtain the overall reaction for process B:

$\mathrm{BrO}_{3}^{-}+4 \mathrm{Ce}^{3+}+\mathrm{CH}_{2}(\mathrm{COOH})_{2}+5 \mathrm{H}^{+} \rightarrow \mathrm{BrCH}(\mathrm{COOH})_{2}+4 \mathrm{Ce}^{4+}+3 \mathrm{H}_{2} \mathrm{O}$.

In process $\mathrm{C}$, cerium(IV) oxidizes bromomalonic acid and malonic acid with the generation of cerium(III) (R9, R10) and liberation of bromide ion (R10, R11), which ultimately terminates the autocatalytic production of $\mathrm{HBrO}_{2}$ and resets the reaction.

$$
\begin{aligned}
& \mathrm{CH}_{2}(\mathrm{COOH})_{2}+6 \mathrm{Ce}^{4+}+2 \mathrm{H}_{2} \mathrm{O} \rightarrow 6 \mathrm{Ce}^{3+}+\mathrm{HCOOH}+2 \mathrm{CO}_{2}+6 \mathrm{H}^{+} \\
& \mathrm{BrCH}(\mathrm{COOH})_{2}+4 \mathrm{Ce}^{4+}+2 \mathrm{H}_{2} \mathrm{O} \rightarrow \mathrm{Br}^{-}+4 \mathrm{Ce}^{3+}+\mathrm{HCOOH}+2 \mathrm{CO}_{2}+5 \mathrm{H}^{+} \\
& \mathrm{Br}_{2}+\mathrm{HCOOH} \rightarrow \mathrm{CO}_{2}+2 \mathrm{Br}^{-}+2 \mathrm{H}^{+}
\end{aligned}
$$

Reaction (R11) is not included in the original FKN mechanism [4], but is included 
in process $\mathrm{C}$ in Refs. [9] and [11]. The most important characteristic of process $\mathrm{C}$ is the number of bromide ions produced per ceric ion consumed in the oxidation of the mixture of malonic and bromomalonic acids.

\subsection{The Mechanism of Light Sensitivity in the Ru(bpy $)_{3}{ }^{2+}$ Catalyzed BZ Reaction}

The $\mathrm{Ru}(\text { bpy })_{3}{ }^{2+}$ catalyzed photosensitive $\mathrm{BZ}$ reaction plays a prominent role in the study of nonlinear phenomena in chemical dynamics. Here, we outline its chemical mechanism elucidated by Kádár et al. [8]. These authors separately investigated the inorganic reaction subset, consisting of $\mathrm{Ru}(\mathrm{II})$, bromate, and sulfuric acid, and the organic reaction subset, consisting of $\mathrm{Ru}(\mathrm{II})$, bromomalonic acid, and sulfuric acid. They found that light affects the $\mathrm{Ru}(\mathrm{bpy})_{3}{ }^{2+}$ catalyzed $\mathrm{BZ}$ reaction via two separate photochemical pathways. The first is the production of bromide ion from the reduction of bromomalonic acid by the photocatalyst, $\mathrm{Ru}(\mathrm{bpy})_{3}{ }^{2+*}$. The second is the generation of bromous acid $\left(\mathrm{HBrO}_{2}\right)$ from the reduction of bromate $\left(\mathrm{BrO}_{3}^{-}\right)$by the excited catalyst $\mathrm{Ru}(\text { bpy })_{3}{ }^{2+*}$.

The primary photochemical process is the absorption of visible light by the $\mathrm{Ru}(\mathrm{bpy})_{3}{ }^{2+}$ complex. The excited state of the complex, $\mathrm{Ru}(\mathrm{II})^{*}$, is an extremely strong reducing agent, as evidenced by the low reduction potential compared to the unexcited complex [12]:

$$
\begin{array}{ll}
\mathrm{Ru}(\mathrm{III})+\mathrm{e}^{-}=\mathrm{Ru}(\mathrm{II}) & \mathrm{E}_{0}=1.26 \mathrm{~V} \\
\mathrm{Ru}(\mathrm{III})+\mathrm{e}^{-}=\mathrm{Ru}(\mathrm{II})^{*} & \mathrm{E}_{0}=-0.86 \mathrm{~V}
\end{array}
$$

Photoinduced bromide production in acidic solution containing bromomalonic acid (BrMA) and $\mathrm{Ru}(\mathrm{II})$ comprises the first pathway acting through the organic subset:

$\mathrm{Ru}(\mathrm{II})+\mathrm{hv} \rightarrow \mathrm{Ru}(\mathrm{II})^{*}$

$\mathrm{Ru}(\mathrm{II})^{*}+\mathrm{BrMA} \rightarrow \mathrm{Ru}(\mathrm{III})+\mathrm{Br}^{-}+$org. prod. 
$\mathrm{Ru}(\mathrm{III})+\mathrm{BrMA} \rightarrow \mathrm{Ru}(\mathrm{II})+\mathrm{Br}^{-}+$org. prod.

The reaction (2.9) is similar to (R10) of the FKN mechanism.

The second pathway occurs at low-intensity illumination when $\mathrm{Ru}(\mathrm{II})^{*}$ reduces bromate in the reaction associated with the inorganic subset:

$\mathrm{Ru}(\mathrm{II})^{*}+\mathrm{BrO}_{3}^{-}+2 \mathrm{H}^{+} \rightarrow \mathrm{BrO}_{2} \cdot+\mathrm{H}_{2} \mathrm{O}+\mathrm{Ru}(\mathrm{III})$,

followed by the autocatalytic cycle $(2.11,2.12)$ similar to the reactions (R5) and (R6) of the FKN mechanism:

$\mathrm{BrO}_{2} \cdot+\mathrm{H}^{+}+\mathrm{Ru}(\mathrm{II}) \rightarrow \mathrm{HBrO}_{2}+\mathrm{Ru}(\mathrm{III})$,

$\mathrm{HBrO}_{2}+\mathrm{BrO}_{3}^{-}+\mathrm{H}^{+} \rightarrow 2 \mathrm{BrO}_{2} \cdot+\mathrm{H}_{2} \mathrm{O}$.

From their studies, the authors concluded that the production of the inhibitor, $\mathrm{Br}^{-}$, is the predominant effect, while the production of the autocatalyst, $\mathrm{HBrO}_{2}$, is of secondary importance. These results provide an explanation of the dependence of light sensitivity on the initial concentration of bromomalonic acid. 
Table 2.1. The FKN mechanism [4]

\begin{tabular}{|c|c|}
\hline Reaction number & Reaction \\
\hline (R1) & $\mathrm{Br}^{-}+\mathrm{HOBr}+\mathrm{H}^{+} \rightleftharpoons \mathrm{Br}_{2}+\mathrm{H}_{2} \mathrm{O}$ \\
\hline (R2) & $\mathrm{Br}^{-}+\mathrm{HBrO}_{2}+\mathrm{H}^{+} \rightarrow 2 \mathrm{HOBr}$ \\
\hline (R3) & $\mathrm{Br}^{-}+\mathrm{BrO}_{3}^{-}+2 \mathrm{H}^{+} \rightarrow \mathrm{HOBr}+\mathrm{HBrO}_{2}$ \\
\hline (R4) & $2 \mathrm{HBrO}_{2} \rightarrow \mathrm{HOBr}+\mathrm{BrO}_{3}^{-}+\mathrm{H}^{+}$ \\
\hline (R5) & $\mathrm{HBrO}_{2}+\mathrm{BrO}_{3}^{-}+\mathrm{H}^{+} \rightleftharpoons 2 \mathrm{BrO}_{2} \cdot+\mathrm{H}_{2} \mathrm{O}$ \\
\hline (R6) & $\mathrm{BrO}_{2} \cdot+\mathrm{Ce}^{3+}+\mathrm{H}^{+} \rightleftharpoons \mathrm{Ce}^{4+}+\mathrm{HBrO}_{2}$ \\
\hline (R7) & $\mathrm{BrO}_{2} \cdot+\mathrm{Ce}^{4+}+\mathrm{H}_{2} \mathrm{O} \rightarrow \mathrm{Ce}^{3+}+\mathrm{BrO}_{3}^{-}+2 \mathrm{H}^{+}$ \\
\hline (R8) & $\mathrm{Br}_{2}+\mathrm{CH}_{2}(\mathrm{COOH})_{2} \rightarrow \mathrm{BrCH}(\mathrm{COOH})_{2}+\mathrm{Br}^{-}+\mathrm{H}^{+}$ \\
\hline (R9) & $\mathrm{CH}_{2}(\mathrm{COOH})_{2}+6 \mathrm{Ce}^{4+}+2 \mathrm{H}_{2} \mathrm{O} \rightarrow 6 \mathrm{Ce}^{3+}+\mathrm{HCOOH}+2 \mathrm{CO}_{2}+6 \mathrm{H}^{+}$ \\
\hline (R10) & $\mathrm{BrCH}(\mathrm{COOH})_{2}+4 \mathrm{Ce}^{4+}+2 \mathrm{H}_{2} \mathrm{O} \rightarrow \mathrm{Br}^{-}+4 \mathrm{Ce}^{3+}+\mathrm{HCOOH}+2 \mathrm{CO}_{2}+5 \mathrm{H}^{+}$ \\
\hline
\end{tabular}




\subsection{The Kinetic Model}

In 1974, Field and Noyes [5] reduced the FKN mechanism [4] to five essential steps, involving three independent chemical intermediates. The resulting model is known as the Oregonator. Here, we use the model as it appeared in [9], which is different from the original Oregonator model proposed by Field and Noyes [5] in the products of each step and the definitions of $\mathrm{A}$ and $\mathrm{B}$, but has identical mass-action kinetic equations describing the three intermediates.

$$
\begin{aligned}
& \mathrm{A}+\mathrm{W} \rightarrow \mathrm{U}+\mathrm{P} \\
& \mathrm{U}+\mathrm{W} \rightarrow 2 \mathrm{P} \\
& \mathrm{A}+\mathrm{U} \rightarrow 2 \mathrm{U}+2 \mathrm{~V} \\
& 2 \mathrm{U} \rightarrow \mathrm{A}+\mathrm{P} \\
& \mathrm{B}+\mathrm{V} \rightarrow \mathrm{hW}
\end{aligned}
$$

Where $\mathrm{A}=\mathrm{BrO}_{3}^{-}, \mathrm{B}=\mathrm{BrMA}[9]$ (or $\left.\mathrm{B}=\mathrm{BrMA}+\mathrm{MA}[13,14]\right), \mathrm{P}=\mathrm{HOBr}, \mathrm{U}=$ $\mathrm{HBrO}_{2}, \mathrm{~V}=\mathrm{Ce}^{4+}$ (or $\left.\mathrm{Me}(\mathrm{ox})\right)$, and $\mathrm{W}=\mathrm{Br}^{-}$. The reactions are numbered as in Ref. $[9]$.

If the steps of the model are all assumed to be irreversible, the kinetic behavior of the Oregonator can be described by the following differential equations:

$$
\begin{aligned}
& d U / d T=k_{1} A W-k_{2} U W+k_{3} A U-2 k_{4} U^{2} \\
& d V / d T=2 k_{3} A U-k_{5} B V \\
& d W / d T=-k_{1} A W-k_{2} U W+h k_{5} B V .
\end{aligned}
$$

where $\mathrm{k}_{1}, \ldots, \mathrm{k}_{5}$ are the rate constants for the five steps, A and B are assumed to be time independent, $\mathrm{U}, \mathrm{V}, \mathrm{W}$ are concentrations in moles $\operatorname{liter}^{-1}\left(\mathrm{M} \mathrm{L}^{-1}\right)$ and $\mathrm{T}$ is time in seconds. These equations have been considerably simplified by Tyson and Fife [9] by introducing dimensionless variables $u, v, w$, and $t$, scaling parameters $\varepsilon, \varepsilon^{\prime}$ and $q$, and the adjustable stoichiometry parameter $f$ :

$$
\begin{aligned}
& u=\left(2 k_{4} / k_{3} A\right) U, v=\left[k_{4} k_{5} B /\left(k_{3} A\right)^{2}\right] V, w=\left(k_{2} / k_{3} A\right) W, t=k_{5} B T, \varepsilon=k_{5} B / k_{3} A, \\
& \varepsilon^{\prime}=2 k_{4} k_{5} B / k_{2} k_{3} A, q=2 k_{1} k_{4} / k_{2} k_{3}, f=2 h .
\end{aligned}
$$


The resulting kinetic equations in dimensionless form are:

$$
\begin{aligned}
\varepsilon d u / d t & =q w-u w+u-u^{2}, \\
d v / d t & =u-v, \\
\varepsilon^{\prime} d w / d t & =-q w-u w+f v .
\end{aligned}
$$

Equations (2.14) are known as the 3-variable Oregonator model. This model can be further reduced to a 2 -variable model by using the order relations $\varepsilon^{\prime} \ll \varepsilon \ll 1$, and $\varepsilon^{\prime}<q \ll 1$, which can be deduced form the measured rate constants for steps (R1)-(R10). Since $w$ changes on a fast time scale, it is in quasi-equilibrium, so that $w=f v /(u+q)$. Substituting this expression into the original system (2.14), we have

$$
\begin{aligned}
\varepsilon d u / d t & =u-u^{2}-f v[(u-q) /(u+q)], \\
d v / d t & =u-v .
\end{aligned}
$$

The two-variable model allows the excitability of the system to be easily analyzed using the nullclines of equations (2.15).

Equations (2.15) describe the chemical reaction in a well-stirred vessel. When the chemical species are allowed to diffuse freely, we must augment equations (2.15) with diffusion terms so that it becomes:

$$
\begin{aligned}
& \frac{\partial u}{\partial t}=D_{u} \nabla^{2} u+\frac{1}{\varepsilon}\left(u-u^{2}-f v \frac{u-q}{u+q}\right), \\
& \frac{\partial v}{\partial t}=D_{v} \nabla^{2} v+u-v .
\end{aligned}
$$

Here, $D_{u}$ and $D_{v}$ are the dimensionless diffusion constants of $u$ and $v$, respectively, and $\nabla^{2}$ is the Laplacian operator. The space scaling is $\mathrm{L}=\left(D_{u} \delta / D_{1}\right)^{1 / 2}=\left(D_{v} \delta / D_{2}\right)^{1 / 2}$ (see 2.4 of this chapter). $D_{1}, D_{2}$ are the diffusion coefficients for $U$ and $V$ respectively, in units of $\mathrm{cm}^{2} \mathrm{~s}^{-1}$. In the experiment, where the catalyst is immobilized in gel, the diffusion of $v$ is absent, and $D_{v}$ is equal to 0 .

In 1990, Krug, Pohlmann, and Kuhnert [10] modified the Oregonator model by introduction of the bromide production corresponding to the influences of oxygen 
and/or light on BZ systems. They named their model the "modified complete Oregonator" (MCO). The MCO model does not include B in reaction M5, and introduces the reaction

$$
\stackrel{\Phi}{\longrightarrow} W
$$

Here $\Phi$ is the velocity of bromide release that takes into account the additional $\mathrm{Br}^{-}$ production induced by external illumination. If we add this reaction to the Oregonator and use Tyson-Fife scaling [9], equation (2.13.c) becomes:

$$
d W / d T=\Phi-k_{1} A W-k_{2} U W+f k_{5} B V
$$

If $\phi=\left[2 k_{4} /\left(k_{3} A\right)^{2}\right] \Phi$, as scaled in the MCO model [10], equation (2.14.c) will become

$$
\varepsilon^{\prime} d w / d t=\phi-q w-u w+f v .
$$

And the kinetics of $w$ can be adiabatically eliminated by the relation

$$
w=\frac{f v+\phi}{u+q} .
$$

If we consider the photosensitive BZ reaction, in which the catalyst is immobilized in a gel, and use Tyson-Fife scaling combined with reaction (2.17), the modified 2-variable Oregonator model becomes:

$$
\begin{aligned}
& \frac{\partial u}{\partial t}=D_{u} \nabla^{2} u+\frac{1}{\varepsilon}\left(u-u^{2}-(f v+\phi) \frac{u-q}{u+q}\right), \\
& \frac{\partial v}{\partial t}=u-v .
\end{aligned}
$$

Depending on the choice of the parameters $f, q, \varepsilon$, and $\phi$, the chemical medium described by this equation can be characterized as bistable, oscillatory or excitable $[15]$. 


\subsection{Dimensionless Equations of the Oregonator Model}

We start with the kinetic equations (2.13):

$$
\begin{aligned}
& d U / d T=k_{1} A W-k_{2} U W+k_{3} A U-2 k_{4} U^{2}, \\
& d V / d T=2 k_{3} A U-k_{5} B V, \\
& d W / d T=-k_{1} A W-k_{2} U W+h k_{5} B V .
\end{aligned}
$$

Let $U=u / \alpha, V=v / \gamma, W=w / \beta, T=t / \delta$, then (2.13.a), (2.13.b), and (2.13.c) will be

$$
\begin{aligned}
\frac{d u}{d t} & =\frac{\alpha}{\delta}\left(k_{1} A \frac{w}{\beta}-k_{2} \frac{u}{\alpha} \frac{w}{\beta}+k_{3} A \frac{u}{\alpha}-2 k_{4} \frac{u^{2}}{\alpha^{2}}\right), \\
\frac{d w}{d t} & =\frac{\beta}{\delta}\left(-k_{1} A \frac{w}{\beta}-k_{2} \frac{u}{\alpha} \frac{w}{\beta}++h k_{5} B \frac{v}{\gamma}\right), \\
\frac{d v}{d t} & =\frac{\gamma}{\delta}\left(-k_{5} B \frac{v}{\gamma}+2 k_{3} A \frac{u}{\alpha}\right) .
\end{aligned}
$$

We organize equations (2.22) as:

$$
\begin{aligned}
\frac{d u}{d t} & =k_{1} A \frac{\alpha}{\delta} \frac{1}{\beta} w-k_{2} \frac{1}{\delta} \frac{1}{\beta} u w+k_{3} A \frac{1}{\delta} u-2 k_{4} \frac{1}{\delta} \frac{1}{\alpha} u^{2}, \\
\frac{d w}{d t} & =-k_{1} A \frac{1}{\delta} w-k_{2} \frac{1}{\delta} \frac{1}{\alpha} u w+h k_{5} B \frac{\beta}{\gamma} \frac{1}{\delta} v, \\
\frac{d v}{d t} & =-k_{5} B \frac{1}{\delta} v+2 k_{3} A \frac{\gamma}{\delta} \frac{1}{\alpha} u .
\end{aligned}
$$

Now let

$$
k_{5} B \frac{1}{\delta}=1, \quad 2 k_{3} A \frac{\gamma}{\delta} \frac{1}{\alpha}=1,
$$

and then

$$
\begin{aligned}
& \delta=k_{5} B, \\
& \frac{\gamma}{\alpha}=\frac{k_{5} B}{2 k_{3} A} .
\end{aligned}
$$

We now introduce $\varepsilon$, $\varepsilon^{\prime}$ to (2.23.a) and (2.23.b), respectively:

$$
\begin{aligned}
\varepsilon \frac{d u}{d t} & =\varepsilon k_{1} A \frac{\alpha}{\delta} \frac{1}{\beta} w-\varepsilon k_{2} \frac{1}{\delta} \frac{1}{\beta} u w+\varepsilon k_{3} A \frac{1}{\delta} u-\varepsilon 2 k_{4} \frac{1}{\delta} \frac{1}{\alpha} u^{2}, \\
\varepsilon^{\prime} \frac{d w}{d t} & =-\varepsilon^{\prime} k_{1} A \frac{1}{\delta} w-\varepsilon^{\prime} k_{2} \frac{1}{\delta} \frac{1}{\alpha} u w+\varepsilon^{\prime} h k_{5} B \frac{\beta}{\gamma} \frac{1}{\delta} v .
\end{aligned}
$$


and setting the following terms in equations (2.26) equal to unity

$$
\begin{aligned}
& k_{2} \frac{1}{\delta} \frac{1}{\beta}=1, \\
& \varepsilon^{\prime} k_{2} \frac{1}{\delta} \frac{1}{\alpha}=1 \\
& \varepsilon k_{3} A \frac{1}{\delta}=1, \\
& 2 \varepsilon k_{4} \frac{1}{\delta} \frac{1}{\alpha}=1
\end{aligned}
$$

allows the parameters to be identified in terms of the rate constants and constant concentrations.

Combining equations (2.28) and (2.24) yields $\varepsilon=\frac{k_{5} B}{k_{3} A}$;

equations $(2.20)$ and $(2.24)$ yield $\alpha=\frac{2 k_{4}}{k_{3} A}$

equations $(2.27)$ and $(2.24)$ yield $\beta=\frac{k_{2}}{k_{3} A}$

equations $(2.27)$ and $(2.28)$ yield $\varepsilon^{\prime}=\varepsilon \frac{\alpha}{\beta}=\frac{2 k_{4} k_{5} B}{k_{2} k_{3} A}$

and equations $(2.25)$ and $(2.32)$ yield $\gamma=\frac{k_{4} k_{5} B}{\left(k_{3} A\right)^{2}}$

The coeffecient of the last term of (2.26.b) becomes $f=2 h$.

Finally, we let $q=\varepsilon k_{1} A \frac{\alpha}{\delta} \frac{1}{\beta}=\varepsilon^{\prime} k_{1} A \frac{1}{\delta}=\frac{2 k_{1} k_{4}}{k_{2} k_{3}}$.

With the parameters as defined above, the dimensionless Oregonator can be written as equations (2.14) or (2.38):

$$
\begin{aligned}
& d u / d t=\left(q w-u w+u-u^{2}\right) / \varepsilon \\
& d v / d t=u-v \\
& d w / d t=(-q w-u w+f v) / \varepsilon^{\prime} .
\end{aligned}
$$


In the following, we derive dimensionless equations for the Oregonator with diffusion of the autocatalyst $u$ and inhibitor $v$. We first write the kinetic equations:

$$
\begin{aligned}
& d U / d T=k_{1} A W-k_{2} U W+k_{3} A U-2 k_{4} U^{2}=F(U, W), \\
& d V / d T=2 k_{3} A U-k_{5} B V=G(U, V) .
\end{aligned}
$$

Defining $D_{1}$ and $D_{2}$ as the diffusion coefficients for $U$ and $V$, the reaction-diffusion equations can be written as:

$$
\begin{aligned}
& \frac{d U}{d T}=F(U, W)+D_{1} \nabla^{2} U, \\
& \frac{d V}{d T}=G(U, V)+D_{2} \nabla^{2} V .
\end{aligned}
$$

With the dimensionless variables defined above, Eqs. (2.40) become:

$$
\begin{aligned}
& \frac{\partial u}{\partial t}=\frac{\alpha}{\delta} F(U, W)+\frac{\alpha}{\delta} D_{1} \nabla^{2} U, \\
& \frac{\partial v}{\partial t}=\frac{\gamma}{\delta} G(U, V)+\frac{\gamma}{\delta} D_{2} \nabla^{2} V .
\end{aligned}
$$

We now consider Eq. (2.41.a) for one spatial dimension, introducing $\varepsilon$ as in (2.26.a):

$$
\varepsilon \frac{\partial u}{\partial t}=f(u, w, q)+\varepsilon \frac{\alpha}{\delta} D_{1} \frac{\partial^{2} U}{\partial X^{2}}
$$

Letting $X=\frac{x}{L}$ and $U=\frac{u}{\alpha}$, we have

$$
\begin{aligned}
\frac{\partial^{2} U}{\partial X^{2}}=\frac{\partial}{\partial X}\left(\frac{\partial U}{\partial X}\right) & =\frac{1}{\alpha} \frac{\partial}{\partial X} \frac{\partial u}{\partial X} \\
& =\frac{L}{\alpha} \frac{\partial}{\partial X}\left(\frac{\partial u}{\partial x}\right)=\frac{L^{2}}{\alpha} \frac{\partial}{\partial x}\left(\frac{\partial u}{\partial x}\right)=\frac{L^{2}}{\alpha} \frac{\partial^{2} u}{\partial x^{2}}
\end{aligned}
$$

Equation (2.42) can now be written as:

$$
\begin{aligned}
& \varepsilon \frac{\partial u}{\partial t}=f(u, w, q)+\varepsilon \frac{\alpha}{\delta} \frac{L^{2}}{\alpha} D_{1} \frac{\partial^{2} u}{\partial x^{2}} \\
& \frac{\partial u}{\partial t}=\frac{1}{\varepsilon} f(u, w, q)+\frac{L^{2}}{\delta} D_{1} \frac{\partial^{2} u}{\partial x^{2}}=\frac{1}{\varepsilon} f(u, w, q)+D_{u} \frac{\partial^{2} u}{\partial x^{2}} .
\end{aligned}
$$

so the dimensionless diffusion coefficient for $u$ is:

$$
D_{u}=\frac{L^{2}}{\delta} D_{1}
$$


For equation (2.41.b), using the same arguments as above, we obtain:

$$
\begin{aligned}
\frac{\partial v}{\partial t} & =\frac{\gamma}{\delta} G(U, V)+\frac{\gamma}{\delta} D_{2} \nabla^{2} V=g(u, v)+\frac{\gamma}{\delta} \frac{L^{2}}{\gamma} D_{2} \frac{\partial^{2} v}{\partial x^{2}} \\
& =g(u, v)+D_{v} \frac{\partial^{2} v}{\partial x^{2}}
\end{aligned}
$$

and the dimensionless diffusion coefficient for $u$ is:

$$
D_{v}=\frac{L^{2}}{\delta} D_{2}
$$

The equations (2.43) and (2.44) provide a relationship between the parameters $\delta, D_{u}\left(\right.$ or $\left.D_{v}\right), D_{1}\left(\right.$ or $\left.D_{2}\right)$, and the space scaling parameter L.

In summary, the two-variable reaction-diffusion Oregonator is given by

$$
\begin{aligned}
& \frac{\partial u}{\partial t}=D_{u} \nabla^{2} u+\frac{1}{\varepsilon}\left(u-u^{2}-f v \frac{u-q}{u+q}\right), \\
& \frac{\partial v}{\partial t}=D_{v} \nabla^{2} v+u-v .
\end{aligned}
$$

For the photosensitive BZ reaction with the catalyst immobilized in a gel, the Oregonator model becomes:

$$
\begin{aligned}
& \frac{\partial u}{\partial t}=D_{u} \nabla^{2} u+\frac{1}{\varepsilon}\left(u-u^{2}-(f v+\phi) \frac{u-q}{u+q}\right), \\
& \frac{\partial v}{\partial t}=u-v .
\end{aligned}
$$

Equation (2.21) comprises the model used in the numerical simulations in this dissertation. Here, we give an example for the parameters. Choosing the rate constant as in refc. $[13,14]$ :

$$
\begin{aligned}
& \mathrm{k}_{1}=2 \mathrm{M}^{-3} \mathrm{~s}^{-1}\left[\mathrm{H}^{+}\right]^{2}, \\
& \mathrm{k}_{2}=10^{6} \mathrm{M}^{-2} \mathrm{~s}^{-1}\left[\mathrm{H}^{+}\right], \\
& \mathrm{k}_{3}=40 \mathrm{M}^{-2} \mathrm{~s}^{-1}\left[\mathrm{H}^{+}\right], \\
& \mathrm{k}_{4}=2 \times 10^{3} \mathrm{M}^{-1} \mathrm{~s}^{-1}, \\
& \mathrm{k}_{5}=0.4 \mathrm{M}^{-1} \mathrm{~s}^{-1},
\end{aligned}
$$


and with the reactant concentrations:

$$
\begin{aligned}
& \mathrm{B}=[\mathrm{BrMA}]+[\mathrm{MA}]=0.12 \mathrm{M}, \\
& \mathrm{A}=\left[\mathrm{BrO}_{3}^{-}\right]=0.33 \mathrm{M}, \\
& {\left[\mathrm{H}^{+}\right]=0.36 \mathrm{M},}
\end{aligned}
$$

we determines the following values of the parameters:

$$
\begin{aligned}
& \epsilon=\mathrm{k}_{5} \mathrm{~B} / \mathrm{k}_{3} \mathrm{~A}=10^{-2}, \\
& \epsilon^{\prime}=2 \mathrm{k}_{4} \mathrm{k}_{5} \mathrm{~B} / \mathrm{k}_{2} \mathrm{k}_{3} \mathrm{~A}=10^{-4}, \\
& \mathrm{q}=2 \mathrm{k}_{1} \mathrm{k}_{4} / \mathrm{k}_{2} \mathrm{k}_{3}=2 \times 10^{-4}, \\
& \delta=\mathrm{k}_{5} \mathrm{~B} \mathrm{~s}{ }^{-1}=0.05 \mathrm{~s}^{-1}, \\
& \mathrm{~L}=\left[\mathrm{k}_{5} \mathrm{~B} /\left(\mathrm{k}_{3} \mathrm{AD}_{1}\right)^{1 / 2}\right] \mathrm{cm}^{-1}=5.88 \mathrm{~cm}^{-1} .\left(\text { if } \mathrm{D}_{u}=\epsilon, \mathrm{D}_{1}=1.5 \times 10^{-5} \mathrm{~cm}^{2} \mathrm{~s}^{-1}\right. \text {.) }
\end{aligned}
$$




\section{$2.5 \quad$ References}

[1] B. P. Belousov, "Oscillation Reaction and Its Mechanism (in Russian)," Sbornik refceratov po Radiacioni Medicine, 145, 1958 meeting (1959).

[2] A. M. Zhabotinsky, "Periodic Liquid-Phase Oxidation Reaction," Dokl. Akad. Nauk SSR 157, 392-395 (1964).

[3] A. M. Zhabotinsky, "A History of Chemical Oscillations and Waves," Chaos 1, 379-386 (1991).

[4] R. J. Field, E. Körös, and R. M. Noyes, "Oscillations in Chemical Systems. II. Thorough Analysis of Temporal Oscillation in the Bromate-Cerium-Malonic Acid System," J. Am. Chem. Soc. 94, 8649-8664 (1972).

[5] R. J. Field and R. M. Noyes, "Oscillations in Chemical Systems. IV. Limit Cycle Behavior in a Model of a Real Chemical Reaction," J. Chem. Phys. 60, 1877-1884 (1974).

[6] R. J. Field and M. Burger, Eds, Oscillations and Travelling Waves in Chemical Systems (Wiley, New York, 1985).

[7] R. Kapral and K. Showalter, Eds, Chemical Waves and Patterns (Kluwer, Dordrecht, 1995).

[8] S. Kàdàr, T. Amemiya, and K. Showalter, "Reaction Mechanism for Light Sensitivity of the $\mathrm{Ru}(\mathrm{bpy})_{3}^{2+}$-Catalyzed Belousov-Zhabotinsky Reaction," J. Phys. Chem. A. 101, 8200-8206 (1997).

[9] J. J. Tyson and P. C. Fife, "Target Patterns in a Realistic Model of the BelousovZhabotinskii Reaction," J. Chem. Phys. 73, 2224-2237 (1980). 
[10] H.-J. Krug, L. Pohlmann, and L. Kuhnert, "Analysis of the Modified Complete Oregonator Accounting for Oxygen Sensitivity and Photosensitivity of BelousovZhabotinsky Systems," J. Phys. Chem. 94, 4862-4866 (1990).

[11] J. J. Tyson, "A Quantitative Account of Oscillations, Bistability and Traveling Waves in the Belousov-Zhabotinskii Reaction" in [6] pp. 93-144.

[12] K. Kalyanasundaram, "Photophysics, Photochemistry and Solar Energy Conversion with Tris(bipyridyl)ruthenium(II) and its Analogs," Coord. Chem. Rev. 46, 159-244 (1982).

[13] J. P. Keener and J. J. Tyson, "Spiral Waves in the Belousov-Zhabotinskii Reaction," Physica D 21, 307-324 (1986).

[14] J. D. Dockery, J. P. Keener, and J. J. Tyson, "Dispersion of Traveling Waves in the Belousov-Zhabotinskii Reaction," Physica D 30, 177-191 (1988).

[15] A. S. Mikhailov, Foundations of Synergetic I: Distributed Active Systems, (2nd edition), (Springer-Verlag, Berlin, 1994). 


\section{Chapter 3}

\section{Dynamic Media}

A great deal of effort has been devoted to the study of complex spatiotemporal patterns during the last few years, which appear when a nonlinear system is driven away from the thermal equilibrium. A variety of systems have been studied, in such diverse contexts as classical mechanics, hydrodynamics, chemistry, material science,

biophysics and solid state physics [1-5]. All these systems are referred to as active media, among which excitable media are particularly important for describing many spatiotemporal patterns in biological systems. In this Chapter, we introduce a classification of active media and the generic features of excitable media, using the BZ reaction as an example system.

\subsection{Classification of Dynamic Media}

In general, dynamical systems are defined by a time evolution equation for the system variables. A typical example is a chemical system represented by a set of nonlinear ordinary differential equations

$$
\frac{d U}{d t}=F(U, R),
$$


for the $n$ components of the state vector,

$$
U(t)=\left\{u_{i}(t)\right\}=u_{1}(t), \ldots, u_{n}(t)
$$

where $F(U, R)$ is a set of nonlinear functions of all variables and depends on a set of control parameters, $R=R_{1}, \ldots, R_{p}$. The instantaneous state of the system at fixed values of $\mathrm{R}$ can be represented by a point in the $n$-dimensional phase space, with coordinates given by (3.2) and the time evolution by a trajectory in that space. In chemical systems, the element $u_{i}(t)$ is the local concentration of the chemical species $i$, and $F(U, R)$ is the kinetic rate equation. A natural extension of (3.1) is a set of partial differential equations (3.3), which incorporates the notion of a spatial coordinate $(x)$

$$
\frac{\partial U(x, t)}{\partial t}=G\left[U, \frac{\partial U}{\partial x}, \ldots, R\right]
$$

where the right-hand side of (3.3) depends on the gradients and higher spatial derivatives of $U$.

The partial differential equations (3.3) describe reaction-diffusion active media, which can be further classified as bistable, excitable, or oscillatory, depending on their stationary states [6]. In bistable media, every component has two stationary states that are stable under small perturbations. Large enough perturbation can, however, trigger transitions between these two steady states. In excitable media, each component typically has a single stationary state that is stable under small perturbations. If the perturbation exceeds a certain threshold, however, this component produces a strong burst of activity. It undergoes a sequence of transitions and later returns to the initial state of rest. An oscillatory medium consists of elements that typically have one unstable steady state and perform stable limit-cycle oscillations.

A special class of active media is a two-variable system, where one of the components is called as an activator $(u)$ and the other as an inhibitor $(v)$ [6]. Generally, an 
activator-inhibitor system is described by the equations

$$
\begin{aligned}
\tau_{u} \frac{\partial u}{\partial t} & =f(u, v)+D_{u} \nabla^{2} u \\
\tau_{v} \frac{\partial v}{\partial t} & =g(u, v)+D_{v} \nabla^{2} v
\end{aligned}
$$

where $\tau_{u}$ and $\tau_{v}$ are the characteristic times for the activator and the inhibitor, respectively. The activator and the inhibitor typically evolve on markedly different characteristic timescales. The fast variable, the activator, is also called a "propagator" or "trigger" variable, while the slow variable, the inhibitor, is called a "controller" or "recovery" variable.

Figures 3.1(a) and (b) present the typical structure and the contour of $f(u, v)$ and $g(u, v)$ in three dimensional space, respectively. At a fixed value of the inhibitor, the function $f(u, v)$ is shown in Fig. 3.1(c). Within a certain range of values of $u$, it has a positive derivative $d f / d u$, which represents an autocatalytic production of the activator $u$. In the regions where the derivative $d f / d u$ is negative, $v$ inhibits any production of $u$. Figure 3.1(d) shows the contours of $f(u, v)$ and $g(u, v)$ in $u, v$ phase plane. The parallel straight lines belong to $g(u, v)$, whereas the curved lines belong to $f(u, v)$. The numbers on the contour lines are the values of $f(u, v)$ and $g(u, v)$, and the lines having the value of zero are the nullclines. The two nullclines divide the phase plane into four regions in which the signs of $d u / d t$ and $d v / d t$ vary between positive and negative, indicating the slope of any trajectory in that region.

As shown in Fig. 3.1(d), an isolated element of an active medium described by Eqs. (3.4) has the nullclines $f(u, v)=0$ and $g(u, v)=0$. Depending on how these two nullclines intersect, the medium can be bistable, oscillatory or excitable. Figure 3.2 shows the three different possibilities defined by the intersections of the nullclines.

Simple stability analysis reveals that if an intersection point lies on the left or on the right branch of the nullcline $d u / d t=0$, where $d f / d u<0$, this stationary state is stable with respect to small perturbations. The stationary state is always unstable if it lies on the middle branch of the nullcline $d u / d t=0$ and if the slope of the nullcline 


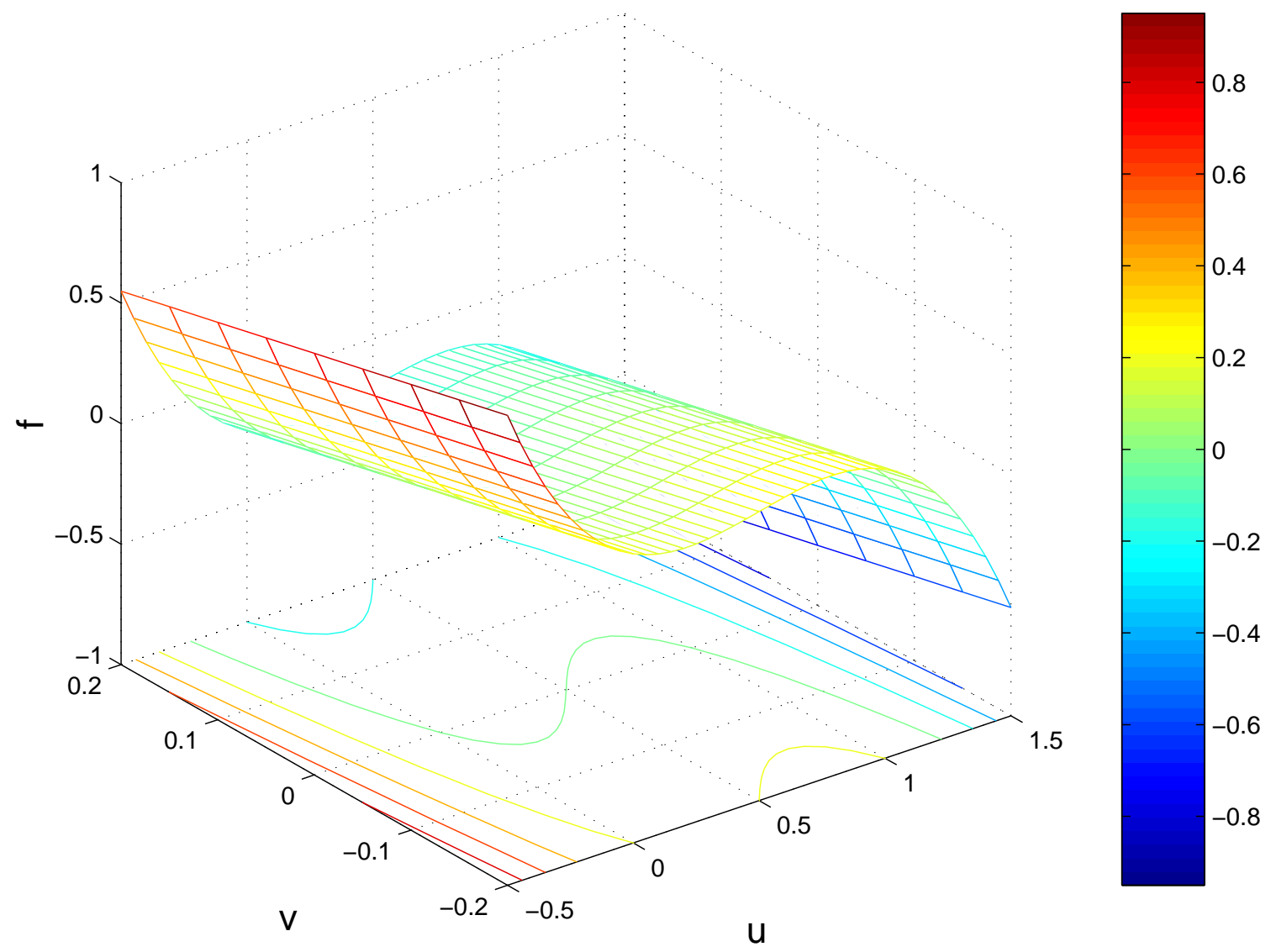

Figure 3.1(a). Typical structure of $f(u, v)$. The contour lines of $f(u, v)$ are in the $u, v$ plane. The color bar indicates the value of $f(u, v)$. 


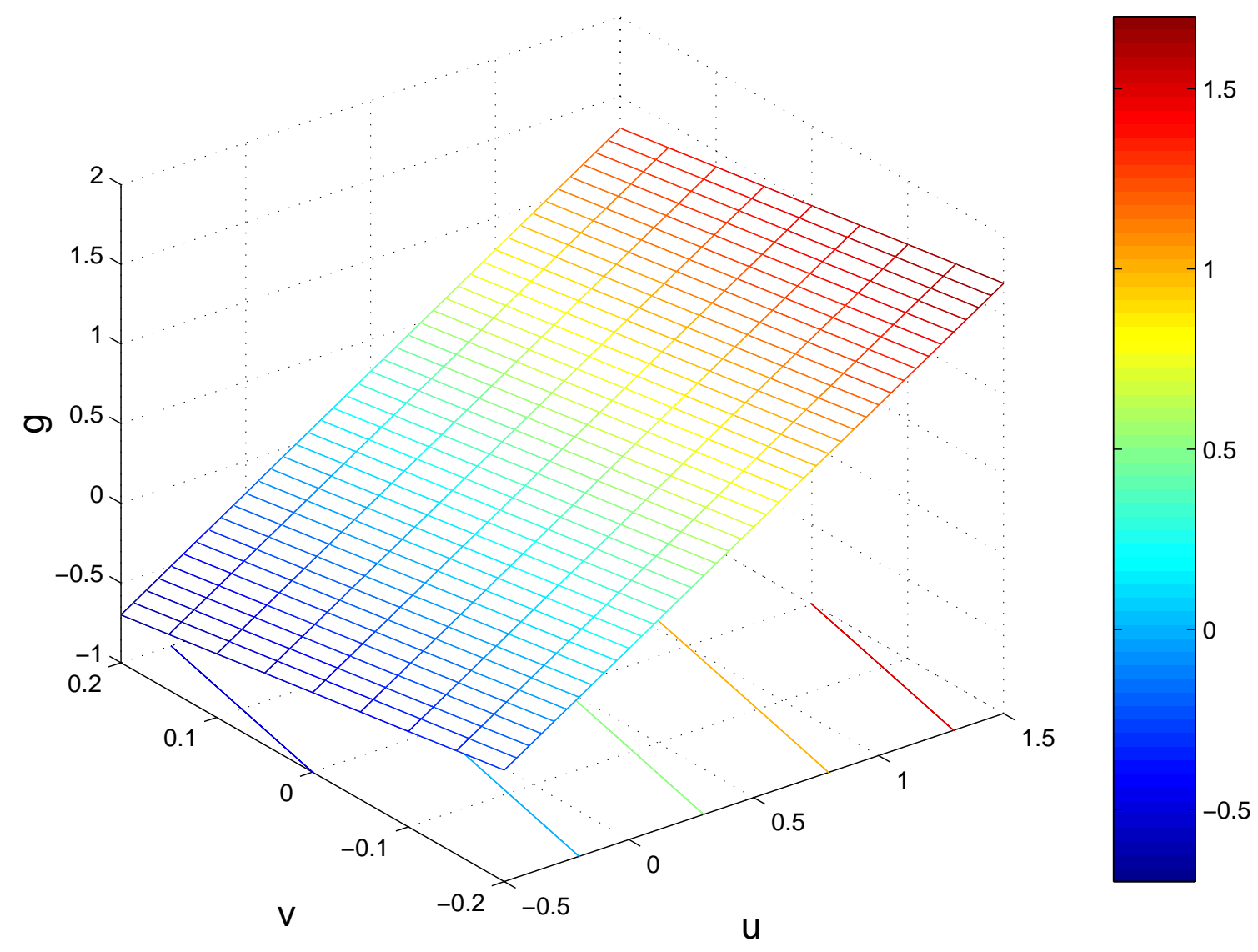

Figure 3.1(b). Typical structure of $g(u, v)$. The contour lines of $g(u, v)$ are in the $u, v$ plane. The color bar indicates the value of $g(u, v)$. 


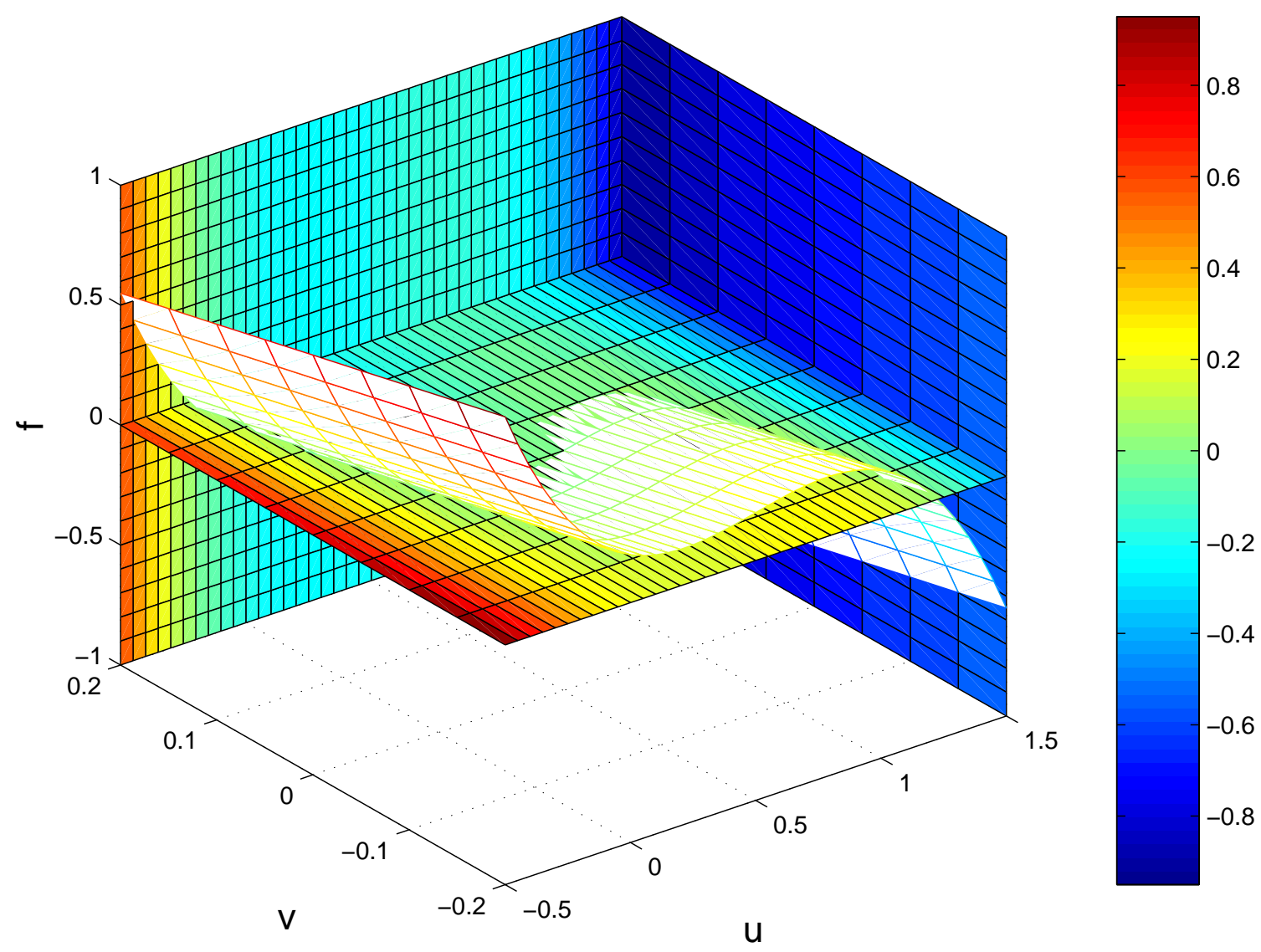

Figure 3.1(c). The structure of function of $f(u, v)$ and the sections on 3 planes: the section of $f(u, v)=0$ cutting $f(u, v)$ into positive and negative regions of $d u / d t$; the section on the $f, u$ plane shows the function $f(u, v)$ at fixed value of $v$. The color changing along the $u$ axis indicates the trend of $d f / d u$. 


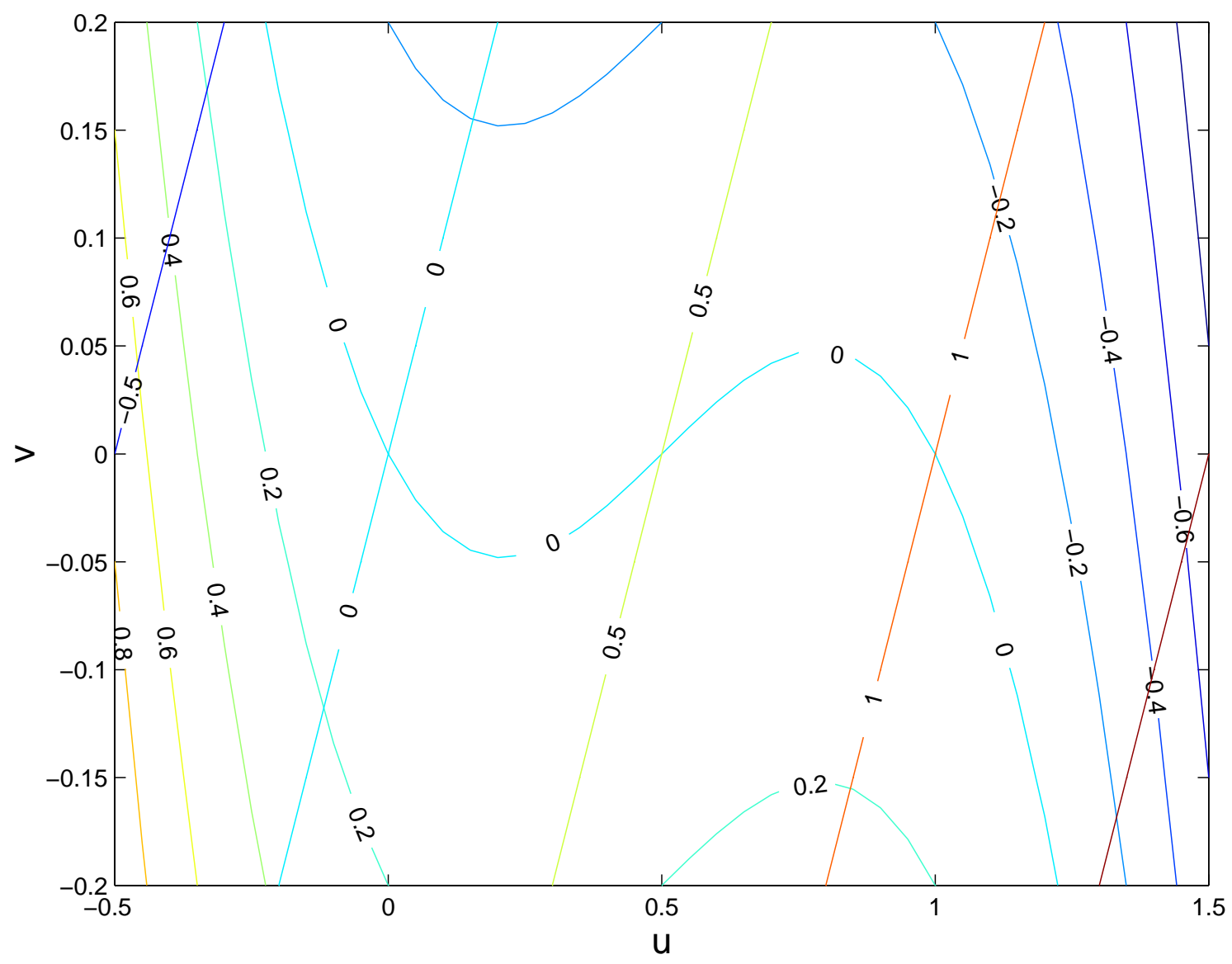

Figure 3.1(d). Contour lines of $f(u, v)$ (curved lines) and $g(u, v)$ (parallel straight lines) in $u, v$ phase plane. The numbers on the lines indicate the values of $f(u, v)$ and $g(u, v)$. The lines with 0 are the nullclines, which divide the phase-plane into four regions with four possible combinations for the signs of $d u / d t$ and $d v / d t$, which establish the slope of trajectories in these regions. 


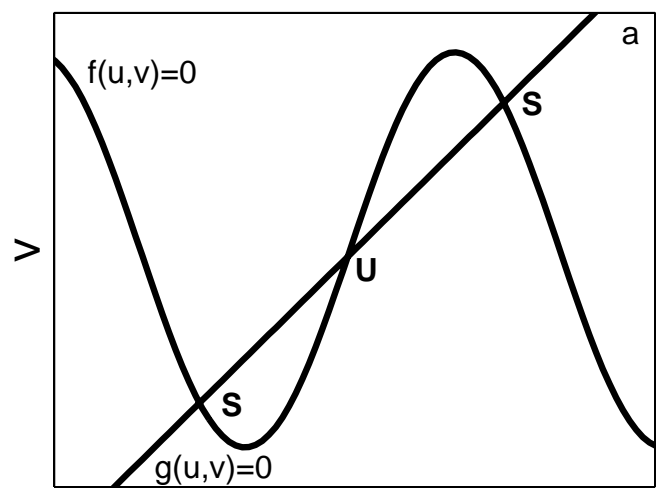

u

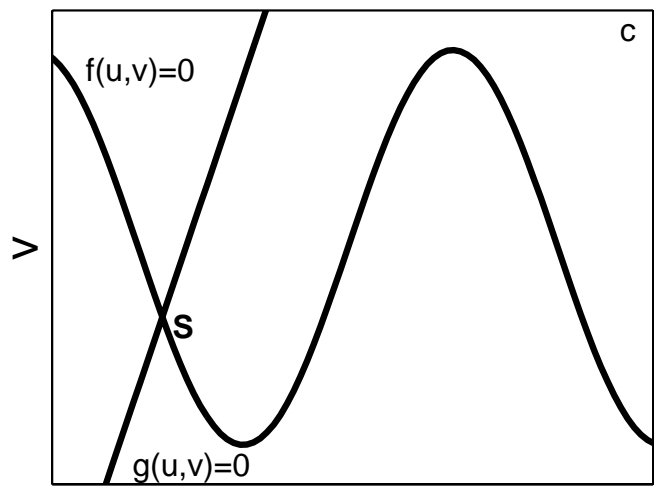

u

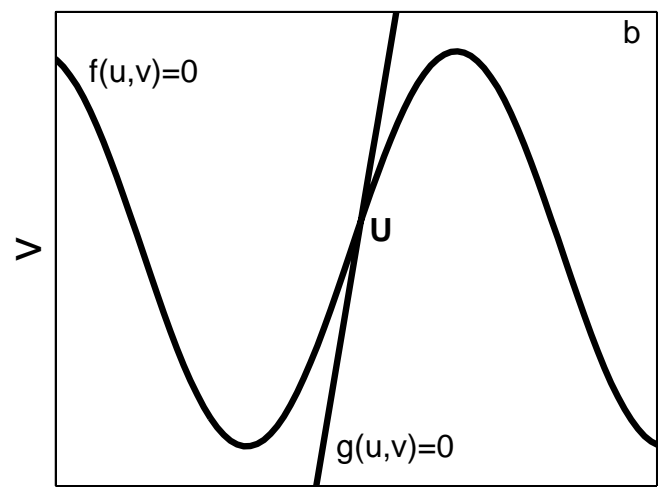

$\mathrm{u}$

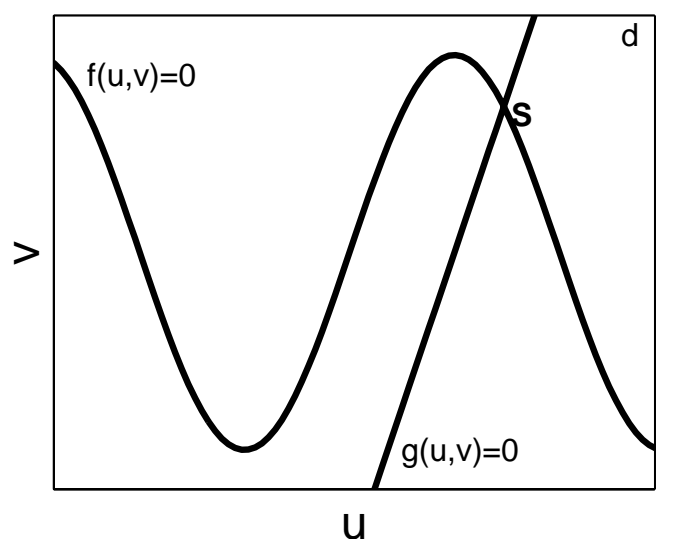

Figure 3.2. The nullclines of an isolated active element. (a) A bistable element, (b) an oscillatory element, (c) and (d) an excitable element. The cubic curve is the $u$ nullcline and the straight line is the $v$ nullcline. "S" indicates a stable steady state and "U" indicates an unstable steady state. 
$d u / d t=0$ is larger than that of the nullcline $d v / d t=0$ at the intersection point. Thus, the element shown in Fig. 3.2(a) is bistable, with two stable steady states and one unstable steady state. When the nullclines intersect as in Fig. 3.2(b), the slope of the nullcline $d u / d t=0$ at the intersection point is less than the slope of the nullcline $d v / d t$ $=0$, the behavior of the stationary state will depend on the ratio $\varepsilon=\tau_{u} / \tau_{v}$ of the characteristic times. If $\varepsilon \ll 1$, the only stationary state of such an element is unstable and the element is oscillatory. For larger values of $\varepsilon$, however, the stationary state may become stable and the element is called Turing-Hopf element [6]. The element shown in Figs. 3.2(c) and (d) has only one stable steady state and is excitable.

\subsection{Excitable Dynamics}

All excitable systems share certain characteristic features. They possess a single stable steady state, also called the rest state, and respond to perturbations in a characteristic manner. Initial states corresponding to small perturbations of the rest state give rise to trajectories that return directly to the rest state in a short time. Perturbations that exceed a certain threshold give rise to trajectories that make a large excursion in the phase space before returning to the rest state. The system is refractory and insensitive to perturbations during theses long excursions. The phaseplane diagram of the two-variable Oregonator model, as shown in Fig. 3.3, illustrates this sequence of events. The subthreshold perturbation A relaxes rapidly to the rest state (see blowup in Fig. 3.3(b)), but the suprathreshold perturbation B undergoes a long excursion before returning to rest, Fig. 3.3(a). First, there is a phase of rapid excitation, BC, during which species $u$ is produced autocatalytically, followed by a period, CD, when the system remains in the excited state. After the excitation phase comes to an end at $\mathrm{E}$, the system is initially absolutely refractory to further excitation and then gradually recovers excitability as it returns to the rest state. In other words, the excitable system is characterized by three states: the rest state (the stable steady 


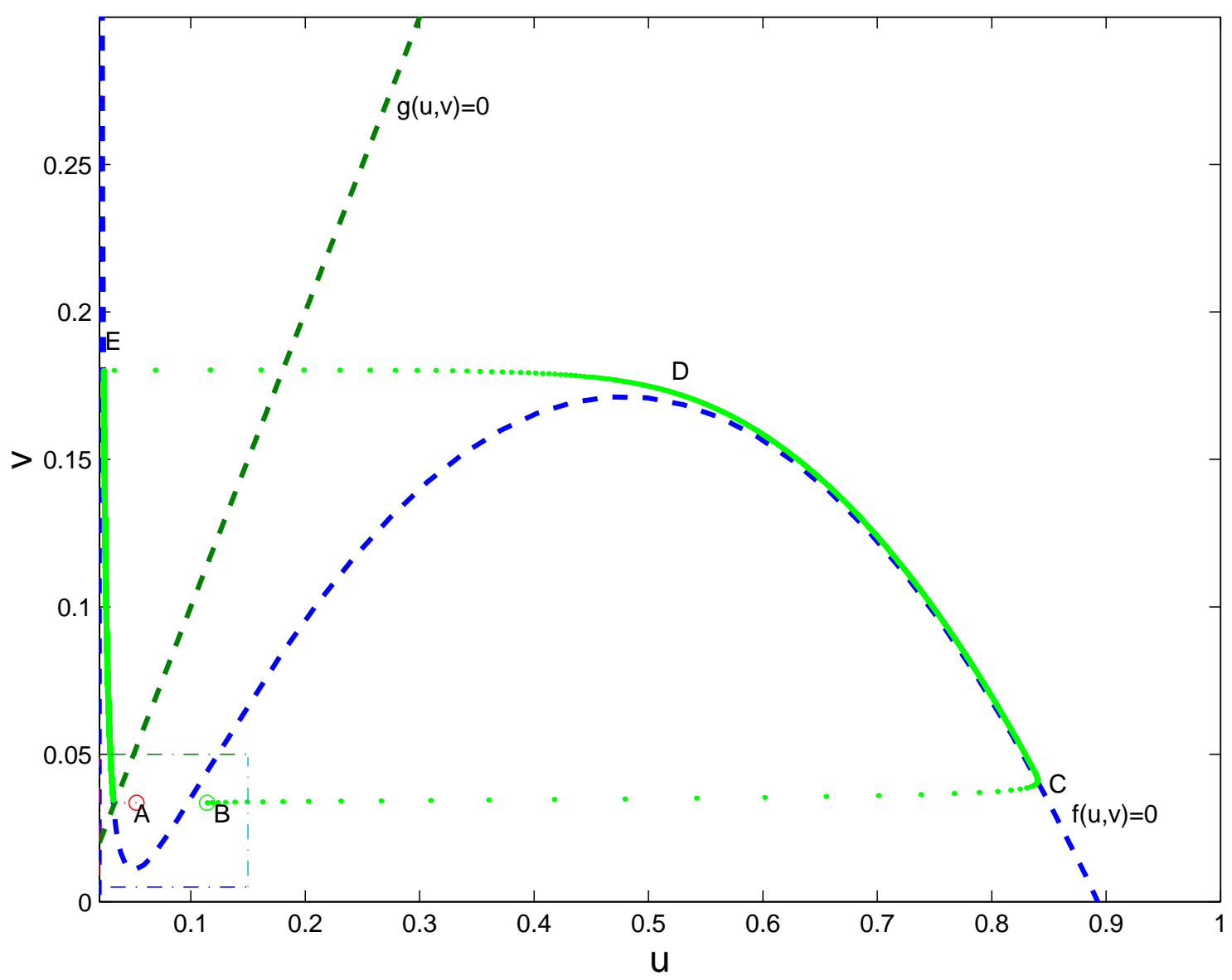

Figure 3.3(a). Typical phase plane for an excitable system, with $u$ and $v$ as the activator and inhibitor of the Oregonator model, Eqs. (3.9). The dashed lines are the nullclines. The short red dotted line is a subthreshold trajectory, and the green dotted line is a suprathreshold trajectory. The red and green circles indicate the initial positions of the trajectories. The region inside the dash-dotted square is magnified in Fig. 3.3(b). The parameters used for simulating the trajectories are $f=1, q=0.02, \phi=0.10, \varepsilon=0.001$. See text for explanation of the trajectories in terms of the points indicated by the letters. 


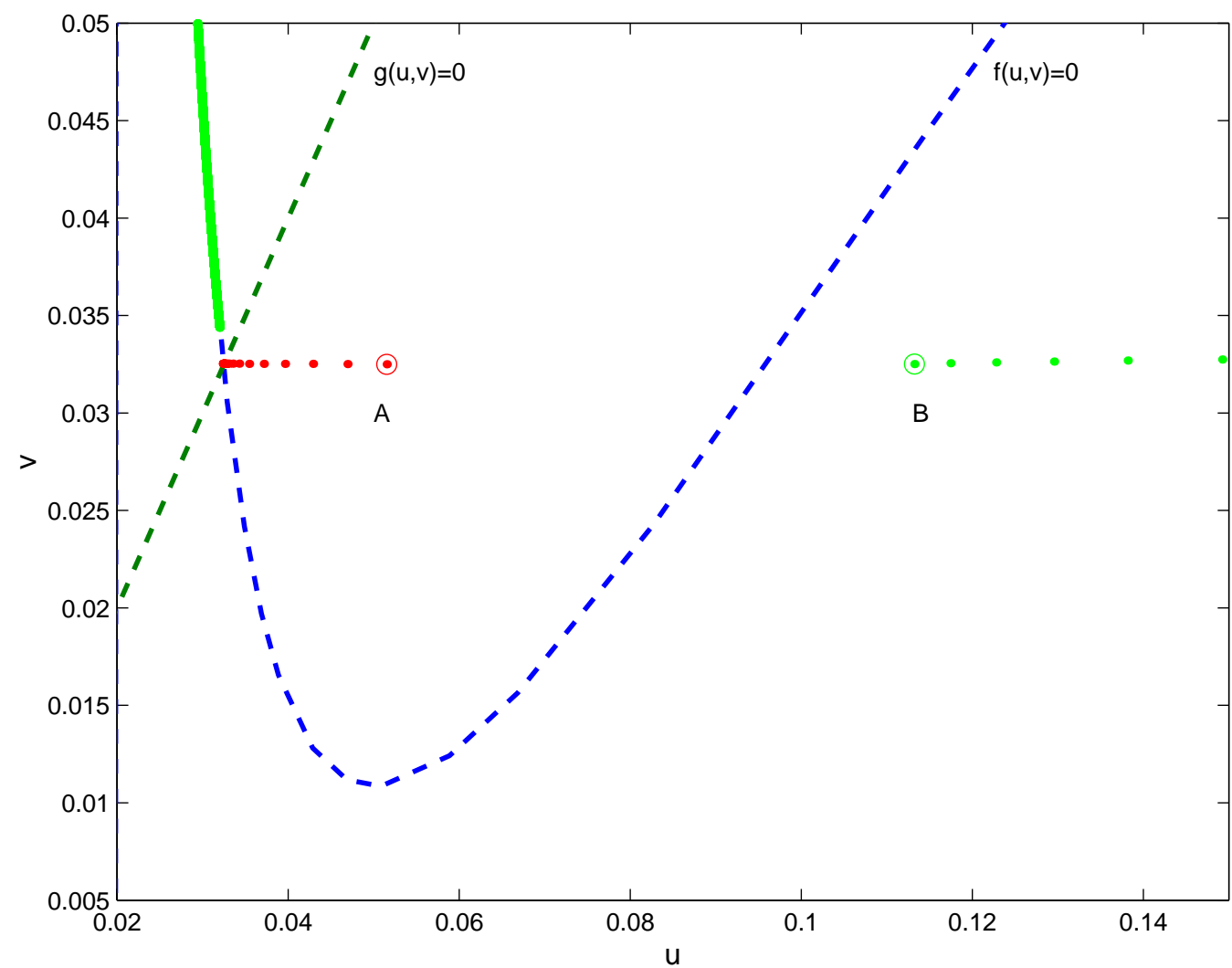

Figure 3.3(b). Magnified region marked by dash-dotted square in Fig. 3.3(a). 
state, also called the quiescent state), the excited state, and the refractory state (also called the recovery state) [7]. Once the system is excited by suprathreshold stimulus, it must go through the refractory period before it can be excited. Such an "excitation cycle" in an excitable system is much like the limit cycle in an oscillatory system. The distance, parallel to $u$ axis, from the rest state to the lower turning point of the three-fold $u$ nullcline is the excitation threshold for a homogeneous excitable system. The higher the threshold, the more difficult it is to excite the system, and, therefore, the lower the excitability of the system. (See Section 3.3 of this Chapter for different excitabilities of BZ systems).

Figures 3.3 and 3.4 indicate the dynamic features of the three branches of the $u$ nullcline. The time interval between the dots representing the perturbed trajectories in Fig. 3.3 is fixed, and, consequently, the distance between the dots represents the rate with which the state of the system changes. The rates for the two "jumps" (from $\mathrm{B}$ to $\mathrm{C}$ and from $\mathrm{D}$ to $\mathrm{E}$ ) are fast, while the rates along the right-hand branch (from $\mathrm{C}$ to D) and the left-hand branch (from E to the rest state) are very slow. The two outer branches represent the slow manifolds. The arrows in Fig.3.4 represent $d u / d t$ and $d v / d t$ at different values of $u$ and $v$. The arrows quantitatively indicate the velocity and the direction of the trajectory at different initial values of $u$ and $v$ in the phase plane according to their sizes and directions, respectively. The longer an arrow, the faster the time evolution at the corresponding points. Figures 3.4(b) and (c) show magnified regions of Fig. 3.4(a). The trajectories converge to the right-hand slow manifold, as shown in Fig. 3.4(b), indicting its attracting character. The trajectories diverge from the middle branch of the $u$ nullcline, as shown in Fig. 3.4(c), indicting its unstable character.

An excitable medium is a spatially distributed system of locally excitable elements. The interaction of neighboring elements by diffusive coupling can produce a number of distinctive types of wave propagation. If a local region of space is perturbed beyond the excitability threshold, then the autocatalytic production of the propagator species 


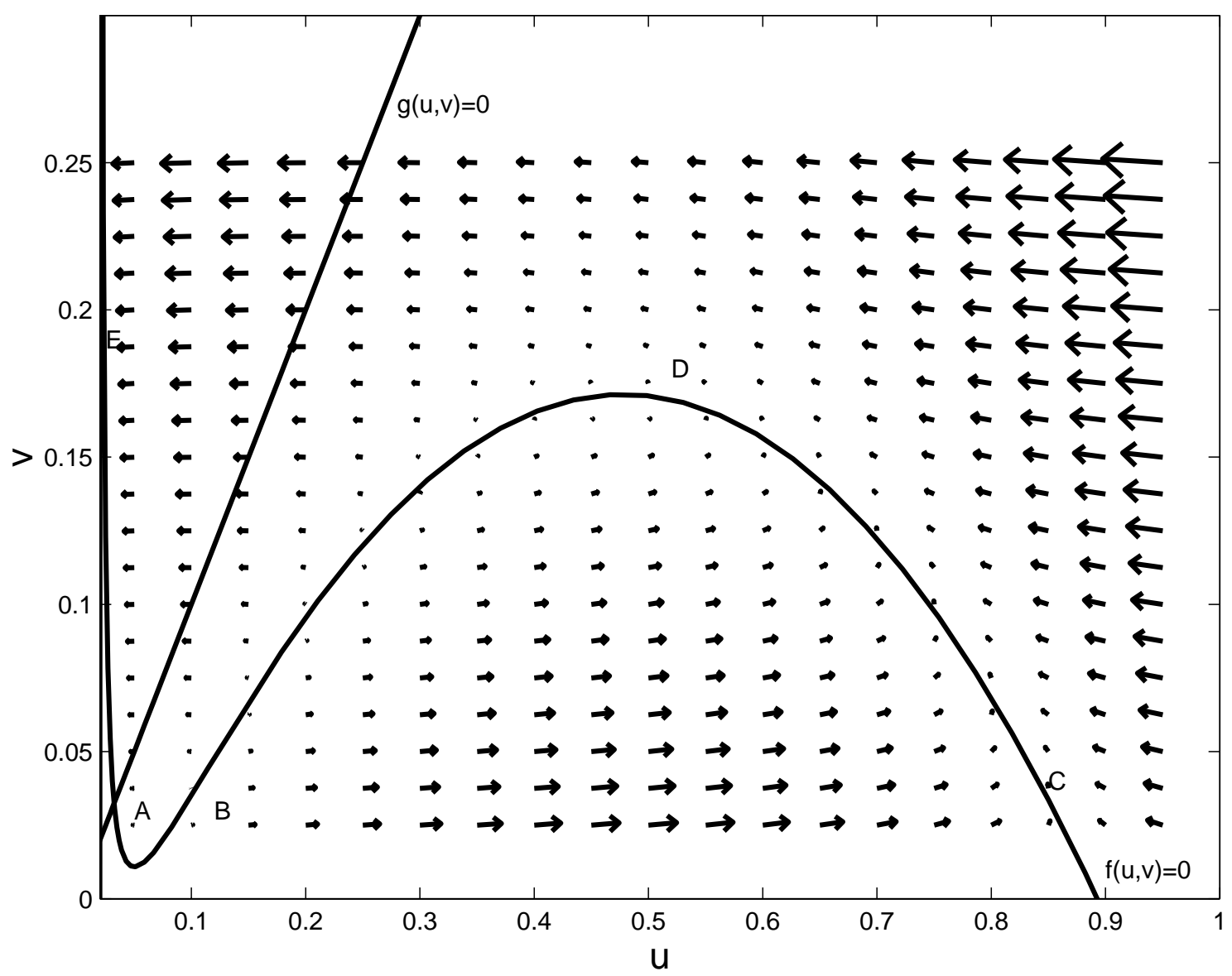

Figure 3.4(a). Trajectories at different initial values of $u$ and $v$ within the unit time $d t$ in the Oregonator model, Eqs. (3.9), with $d t=0.001$. The other parameters are the same as in Fig. 3.3. The solid lines are the nullclines. 


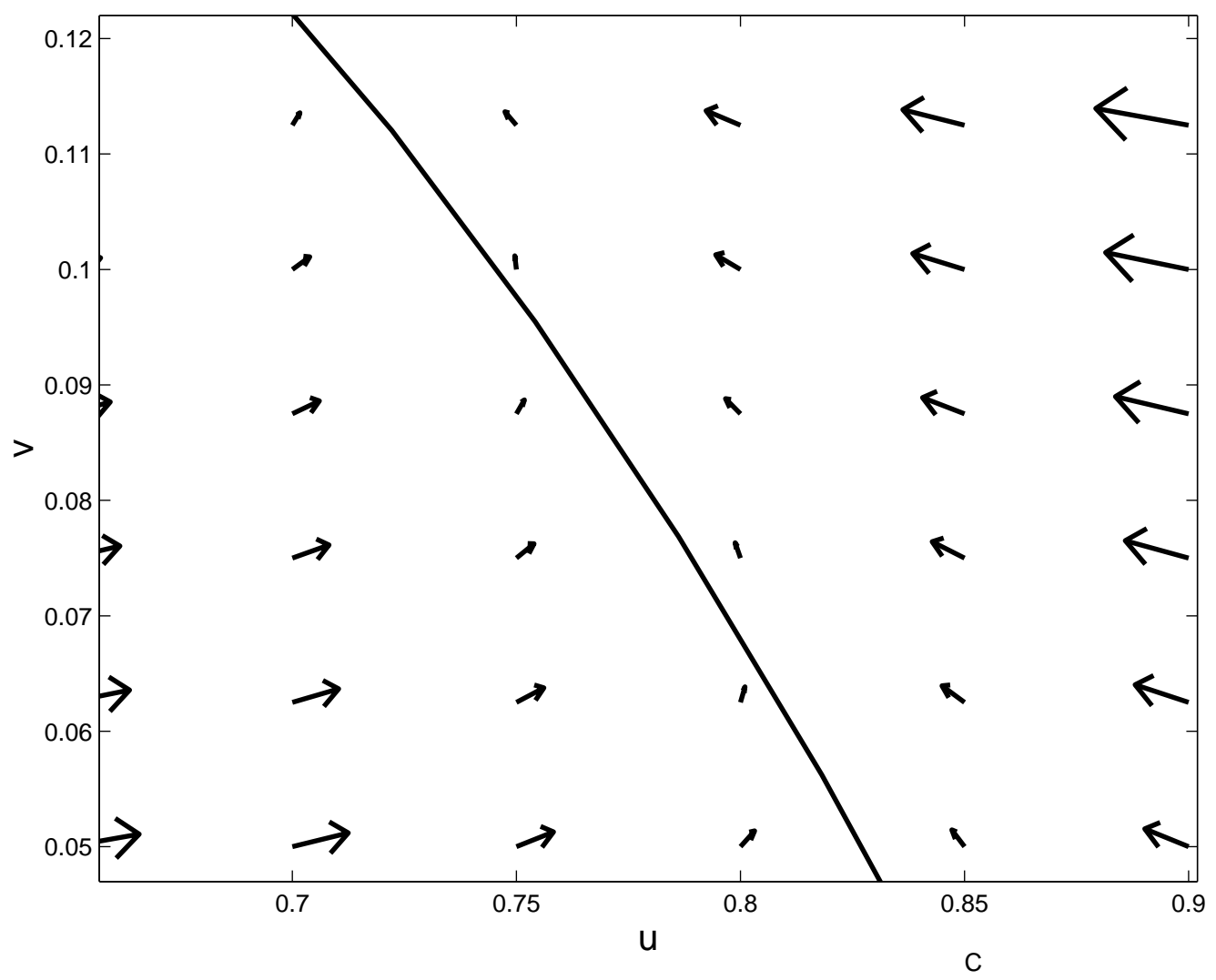

Figure 3.4(b). The magnified trajectories converge along the right-hand branch of the $u$ nullcline in Fig. 3.4(a). 


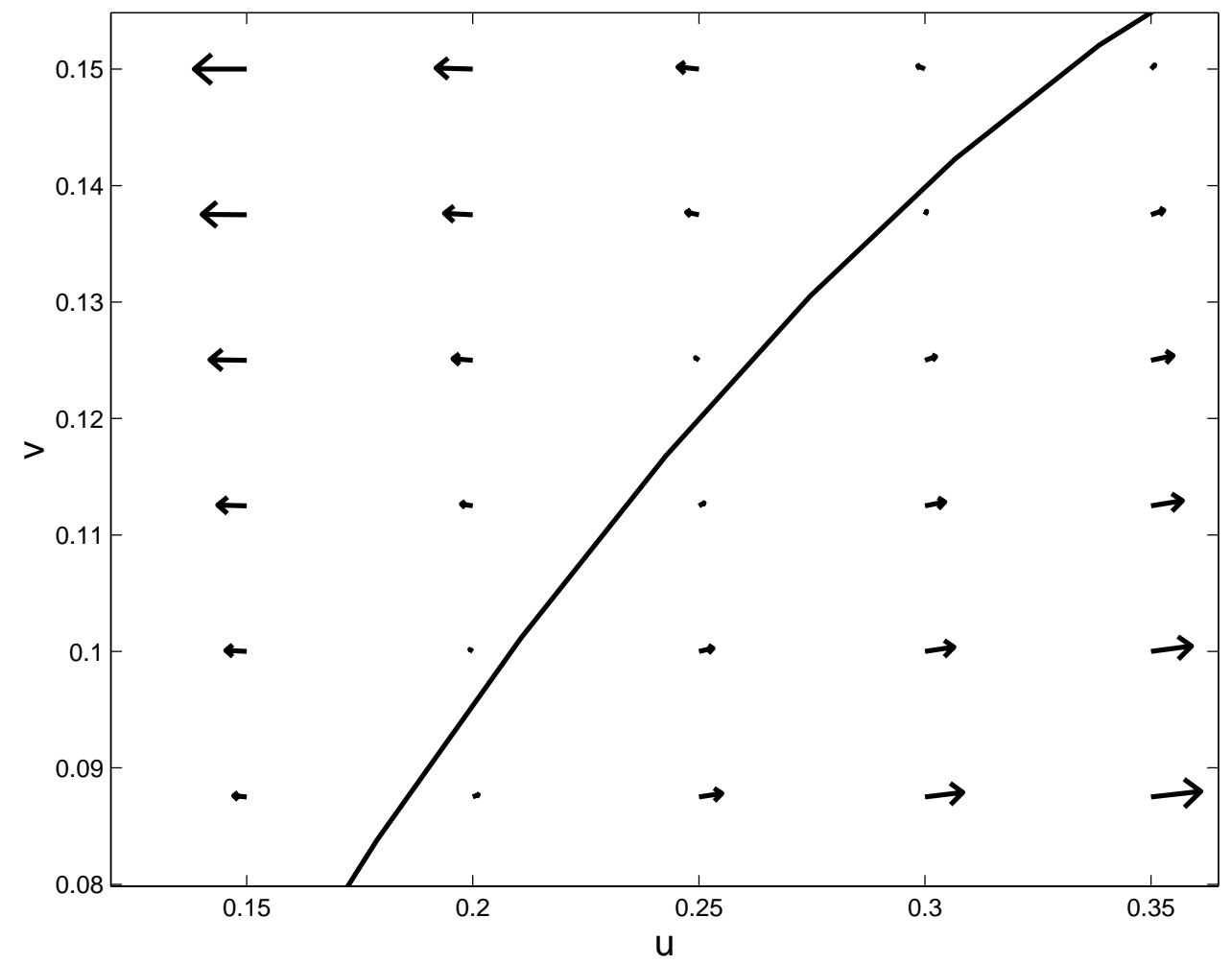

Figure 3.4(c). The magnified trajectories diverge along the middle branch of the $u$ nullcline in Fig. 3.4(a). The $v$ nullcline is omitted here. 
$u$ in that region causes its concentration to increase. Diffusing out into neighboring regions, it will cause the neighboring regions to exceed the threshold and thus cause the excitation to spread out. In one spatial dimension, one would observe a wave train of impulses. Two-dimensional excitable media exhibit two topologically distinct patterns: expanding concentric circular waves, called target patterns, and rotating spiral waves. In three dimensions, the corresponding spatiotemporal structures are expanding concentric spherical waves and rotating scroll waves.

There are many examples of excitable media, which support propagating waves of chemical, physical or biological activity. The most famous is the axonal membrane [8], which can support propagating electrical signals and was first characterized by Hodgkin and Huxley [9]. Other examples of biological excitable media include cardiac muscle [10-14], among which epicardial, ventricular, and atrial muscle are useful for the study of the mechanism of fibrillation and tachycardia. Neuronal tissue supporting waves of spreading depression, which has been studied in rat cerebral cortex [15] and chicken retina [16], has provided insights into the mechanism of migraine disorder [17]. Another example of biochemical media includes the social amoebae Dictyostelium discoideum [18-20], a slime mold that supports waves of cyclic adenosine 3',5'-monophosphate (cyclic AMP) activity. Another biological example is the Xenopus laevis oocyte [21], an intracellular milieu for studying $\mathrm{Ca}^{2+}$ signaling. Examples of chemical excitable systems including the $\mathrm{BZ}$ reaction $[1,5,22,23]$ and the $\mathrm{CO}$ oxidation on single crystal $\mathrm{Pt}(110)$ [24,25], which give rise to many kinds of spatiotemporal patterns in the excitable media. There are also many examples of macroscopic excitable media such as epidemic spreading in population biology [26-28] and spiral galaxies in the celestial system, whose rotating arms can be treated as traveling waves of star formation in an excitable medium of interstellar gas and dust [29,30]. Table 3.1 shows a comparison of some of the examples of excitable media by identifying their characteristic state variables, $u$ and $v[2,31]$. Among these excitable media, the chemical systems have been most extensively used to study the properties 
of excitable media. One of the main advantages of chemical systems is their ability to be controlled and their simplicity in comparison with biological or celestial systems. Among chemical systems, the BZ reaction is the most widely studied excitable chemical medium. 
Table 3.1. State variables of some representative excitable media $[2,31]$

\begin{tabular}{lll}
\hline System & Activator & Inhibitor \\
\hline BZ reaction & bromous acid & oxidized catalyst \\
CO oxidation & coverage of the absorbed CO & structural change of the surface \\
Neuromuscular tissue & membrane potential & ionic conductance \\
Dictyostelium discoideum & cyclic AMP & membrane receptor \\
Epidemics & infectious agent & level of immunity \\
Spiral galaxies & density of molecular cloud & temperature of molecular cloud \\
\hline
\end{tabular}




\subsection{Excitable Media in the BZ Reaction}

An active medium can exhibit different types of spatiotemporal behavior: homogenous oscillations, travelling wave fronts, rotating spirals and target patterns. The BZ reaction $[1-3,5,32]$ is one of the most thoroughly investigated systems demonstrating these types of behaviors. In the BZ reaction, the role of activator, $u$, is played by bromous acid, $\mathrm{HBrO}_{2}$, while the role of inhibitor, $v$, is played by the oxidized form of the metal-ion catalyst. The two-variable Oregonator model [33] describes the temporal dynamics of the BZ reaction:

$$
\begin{aligned}
(\varepsilon) d u / d t & =u-u^{2}-f v[(u-q) /(u+q)]=f(u, v), \\
d v / d t & =u-v=g(u, v) .
\end{aligned}
$$

The nullclines of $u(d u / d t=0)$ and $v(d v / d t=0)$ are given by

$$
\begin{aligned}
& v(u)=\frac{1}{f}\left(\frac{q+u}{q-u}\left(u^{2}-u\right)\right), \\
& v(u)=u .
\end{aligned}
$$

The values of the parameters $\varepsilon, q$ and $f$ determine the dynamic behavior of the system; however, we will focus on the parameter $f$. The steady state, which occurs at the intersection of the nullclines, and the maximum and minimum of the $u$ nullcline are determined by $f$. For $q \ll 1$, when $f$ falls into the range

$$
\frac{1}{2}<f<1+\sqrt{2}
$$

the system is oscillatory. Systems with $f$ only slightly in excess of $1+\sqrt{2}$ are nonoscillatory but excitable [32]. The irreversible Oregonator with flow terms [35,36] modeling the $\mathrm{BZ}$ reaction in a CSTR and a so-called cross-shaped phase diagram have been used to analyze the temporal dynamics of the BZ reaction.

In the photosensitive BZ reaction, the dynamic features of the system depend not only on $f$ but also on $\phi$. The modified Oregonator model [34] for the photosensitive 
BZ reaction (Eqs. (2.21) without diffusion term) is:

$$
\begin{aligned}
\frac{d u}{d t} & =\frac{1}{\varepsilon}\left(u-u^{2}-(f v+\phi) \frac{u-q}{u+q}\right), \\
\frac{d v}{d t} & =u-v .
\end{aligned}
$$

The nullclines of $u$ and $v$ in the photosensitive BZ reaction are given by

$$
\begin{aligned}
& v(u)=\frac{1}{f}\left(\frac{q+u}{q-u}\left(u^{2}-u\right)-\phi\right), \\
& v(u)=u .
\end{aligned}
$$

When $\phi=0$, Eqs. (3.9) are the same as Eqs. (3.6). The nullclines are shown in Fig. 3.5. According to the FKN mechanism, process A occurs when the system is close to the left-hand branch of the $u$ nullcline; process B occurs near the right-hand branch, and process $\mathrm{C}$ occurs at high values of $v$. Figures 3.3 and 3.4 demonstrate the dynamic features of the photosensitive BZ reaction with the parameters $f=1$, $q=0.02, \phi=0.10$. Nullclines with different dynamic features can be controlled by different values of $\phi$ for a fixed value of $f$, as shown in Fig. 3.5. The excitability of the system can be adjusted through varying the light intensity $\phi$, as seen in panels (a)-(d). Varying $\phi$ shifts the nullcline of $u$ up or down, generating an unstable steady state and thus the onset of oscillatory behavior. It also can change the excitability of the excitable system, with the larger the value of $\phi$, the lower the excitability.

In the Chapter "Synchronization of Spatiotemporal Patterns in Locally Coupled Excitable Media" of this dissertation, we use the following parameters for the numerical simulations: $q=0.0015, f=1, \phi=0.035$ and $q=0.002, f=3.5, \phi=0.01$. The corresponding excitable media are described by Figs. 3.6 and 3.7, respectively. The inserts of Fig. 3.6 and Fig. 3.7 show the details of the nullclines when $u$ close to the origins. 

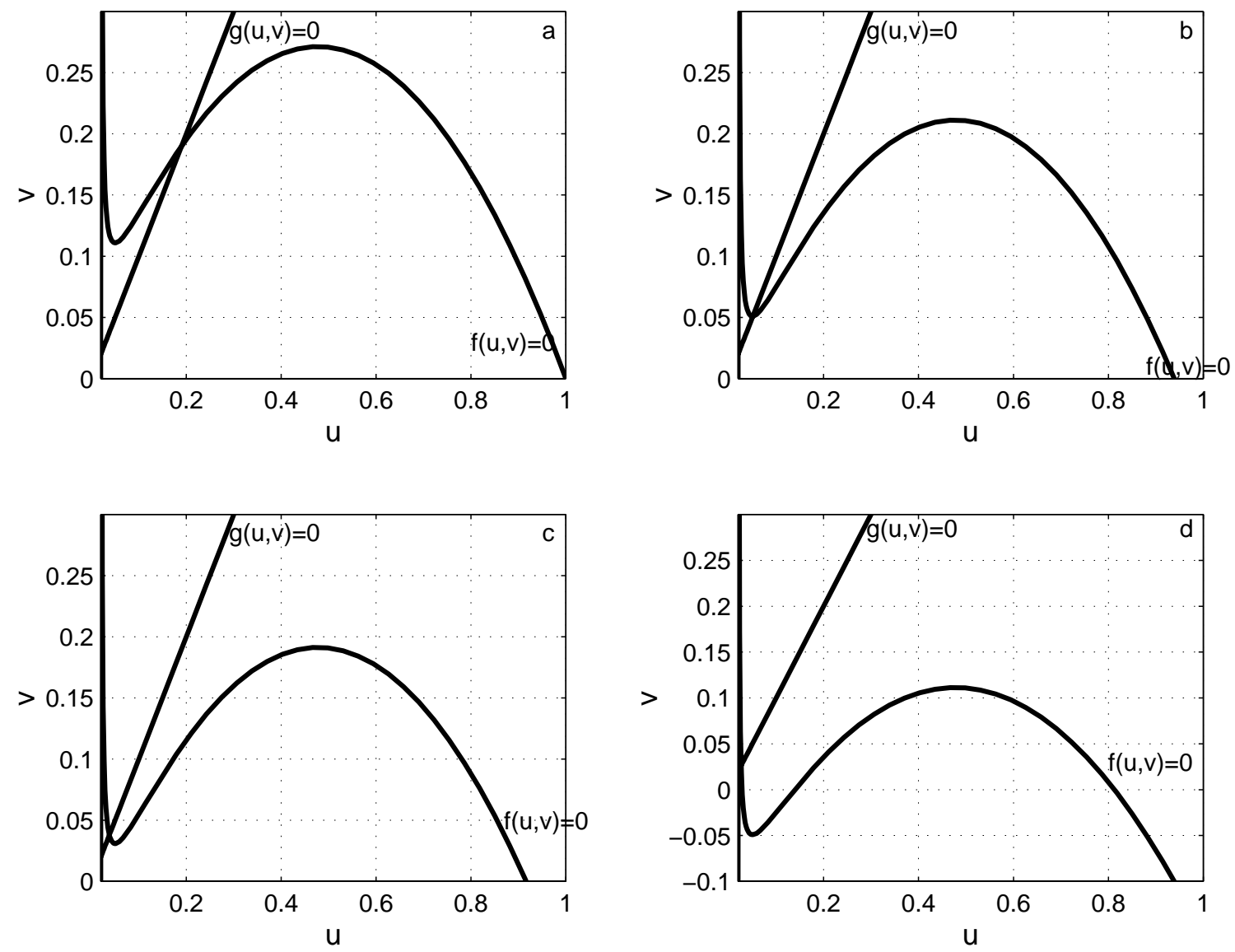

Figure 3.5. The effect of light intensity on the excitability of the photosensitive BZ reaction. The nullclines are plotted for Eqs. (3.9) with the parameters $q=0.02, f=1$ and different $\phi . \phi=0$ (a), $\phi=0.06$ (b), $\phi=0.08$ (c), and $\phi=0.16$ (d). 


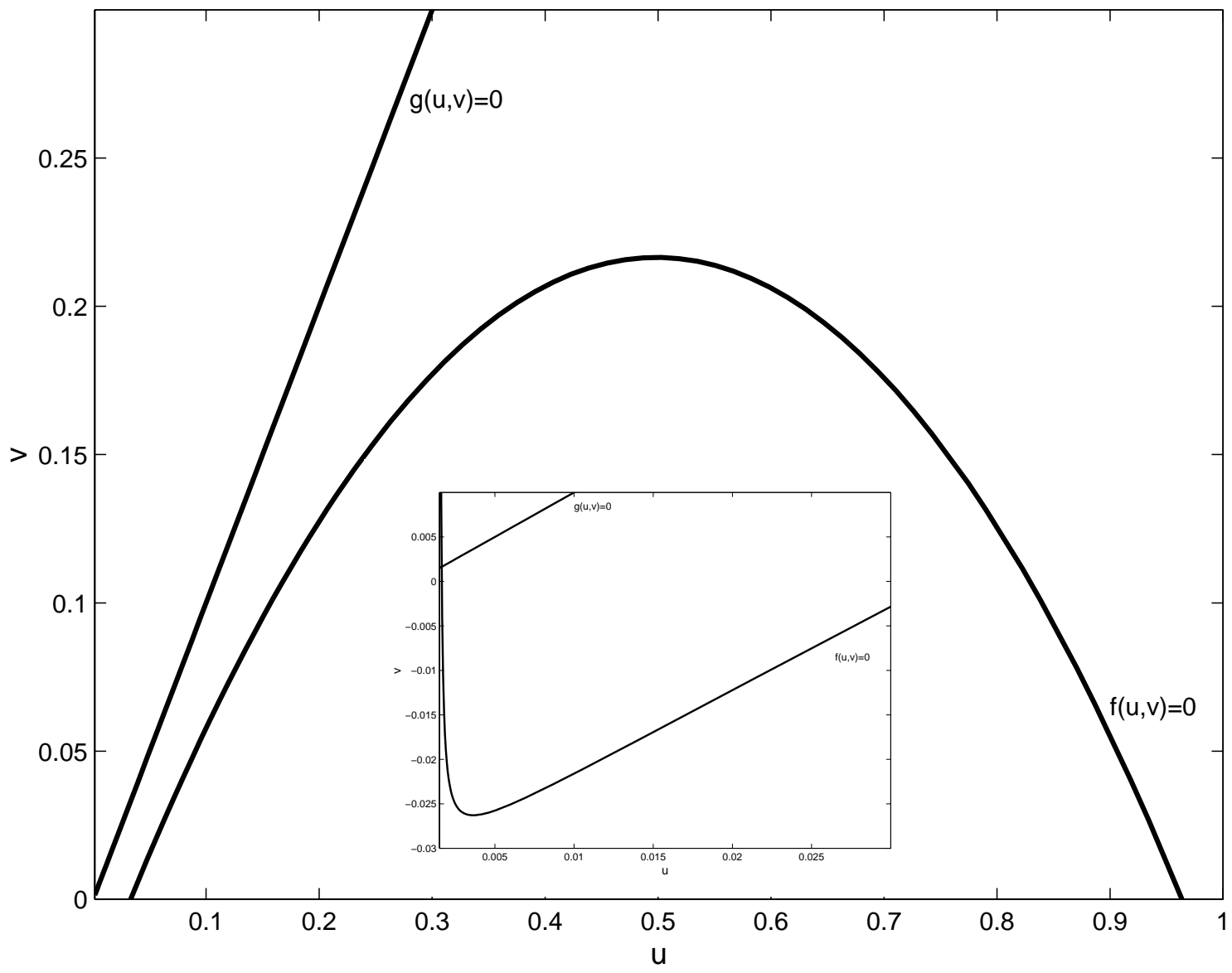

Figure 3.6. Nullclines for the photosensitive BZ reaction. The nullclines of $u$ and $v$ are plotted for Eqs. (3.9), with the parameters $q=0.0015, f=1, \phi=0.035$. The insert shows the details of the nullclines when $u$ close to the origin. 


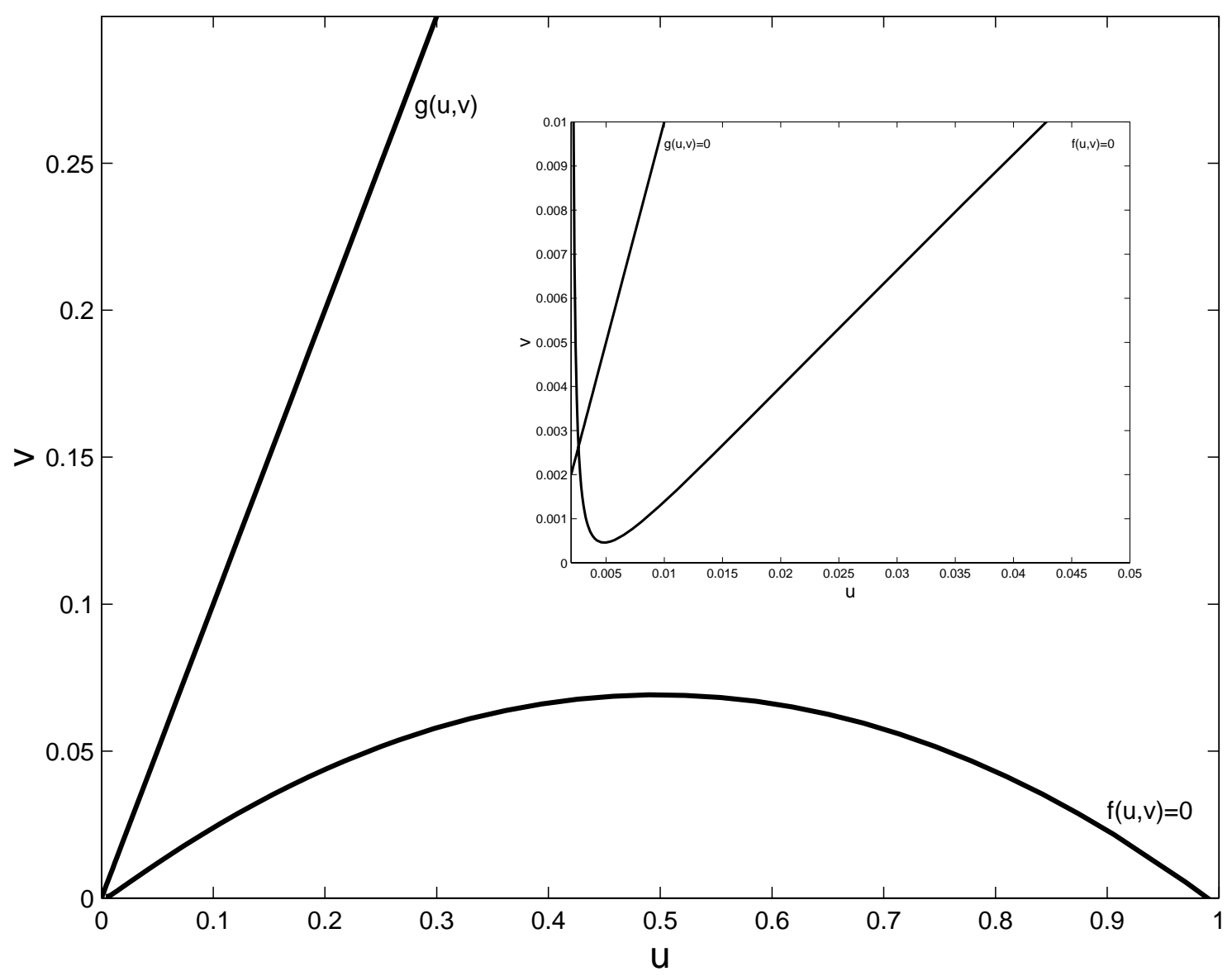

Figure 3.7. Nullclines for the photosensitive BZ reaction. The nullclines of $u$ and $v$ are plotted for Eqs. (3.9), with the parameters $q=0.002, f=3.5, \phi=0.01$. The insert shows the details of the nullclines when $u$ close to the origin. 


\subsection{References}

[1] R. J. Field, and M. Burger, Eds, Oscillations and Travelling Waves in Chemical Systems (Wiley, New York, 1985).

[2] J. J. Tyson and J. P. Keener, "Single Perturbation Theory of Traveling Waves in Excitable Media (A Review)," Physica D 32, 327-361 (1988).

[3] M. C. Cross and P. C. Hohenberg, "Pattern Formation Outside of Equilibrium," Rev. Mod. Phys. 65, 851-1112 (1993).

[4] S. C. Müller, P. Coullet, and D. Walgraef, "From Oscillations to Excitability: A Case Study in Spatially Extended Systems," Chaos 4, 439-442 (1994).

[5] R. Kapral and K. Showalter, Eds, Chemical Waves and Patterns (Kluwer, Dordrecht, 1995).

[6] A. S. Mikhailov, Foundations of Synergetic I: Distributed Active Systems, (2nd edition), (Springer-Verlag, Berlin, 1994).

[7] P. Jung, A. Cornell-Bell, F. Moss, S. Kádár, J. Wang, and K. Showalter, "Noise Sustained Waves in Subexcitable Media: From Chemical Waves to Brain Waves," Chaos 8, 567-575 (1998).

[8] J. Rinzel, "Discussion: Electrical Excitability of Cells, Theory and Experiment: Review of the Hodgkin-Huxley Foundation and an Update," B. Math. Biol. 52, 5-23 (1990).

[9] A. L. Hodgkin and A. F. Huxley, "A Quantitative Description of Membrane Current and Its Application to Conduction and Excitation in Nerve," J. Physiol. 117, 500-544 (1952). 
[10] M. A. Allessie, F. I. M. Bonke, and F. J. G. Schopman, "Circus Movement in Rabbit Atrial Muscle as a Mechanism of Tachycardia," Circ. Res. 33, 54-62 (1973).

[11] M. A. Allessie, F. I. M. Bonke, and F. J. G. Schopman, "Circus Movement in Rabbit Atrial Muscle as a Mechanism of Tachycardia. II. The Role of Nonuniform Recovery of Excitability in the Occurrence of Unidirectional Block, as Studied with Multiple Microelectrodes," Circ. Res. 39, 168-177 (1976).

[12] M. A. Allessie, F. I. M. Bonke, and F. J. G. Schopman, "The "Leading Circle" Concept: A New Model of Circus Movement in Cardiac Tissue without the Involvement of an Anatomical Obstacle," Circ. Res. 41, 9-18 (1977).

[13] A. T. Winfree, When Time Breaks Down, (Princeton University Press, Princeton, 1987).

[14] J. M. Davidenko, A. V. Pertsov, R. Salomonsz, W. Baxter, and J. Jalife, "Stationary and Drifting Spiral Waves of Excitation in Isolated Cardiac Muscle," Nature 355, 349-351 (1992).

[15] V. I. Koroleva and J. Bureš, "Circulation of Cortical Spreading Depression around Electrically Stimulated Area and Epileptic Foci in the Neocortex of Rats," Brain Res. 173, 209-215 (1979).

[16] N. A. Gorelova and J. Bureš, "Spiral Waves of Spreading Depression in the Isolated Chicken Retina," J. Neurobiol. 14, 353-363 (1983).

[17] M. Lauritzen, "Cortical Spreading Depression in Migraine," Cephalalgia 21, 757$760(2001)$.

[18] A. J. Durston, "Dictyostelium discoideum Aggregation Field as Excitable Media," J. Theor. Biol. 42, 483-504 (1973). 
[19] K. J. Tomchik and P. N. Devreotes, "Adenosine 3',5'-Monophosphate Waves in Dictyostelium discoideum: A Demonstration by Isotope Dilution-Fluorography," Science 212, 443-446 (1981).

[20] F. Siegert and C. Weijer, "Digital Image Processing of Optical Density Wave Propagation in Dictyostelium discoideum and Analysis of the Effects of Caffeine and Ammonia," J. Cell Sci. 93, 325-335 (1989).

[21] J. Lechleiter, S. Girard, E. Peralta, and D. Clapham, "Spirals Calcium Wave Propagation and Annihilation in Xenopus laevis Oocytes," Science 252, 123-126 (1991).

[22] A. Z. Zaikin and A. Zhabotinsky, "Concentration Wave Propagation in TwoDimensional Liquid-Phase Self-Oscillating System," Nature 225, 535-537 (1970).

[23] A. T. Winfree, "Spiral Waves of Chemical Activity," Science 175, 634-636 (1972).

[24] S. Jakubith, H. H. Rotermund, W. Engel, A. von Oertzen, and G. Ertl, "Spatiotemporal Concentration Patterns in a Surface Reaction: Propagating and Standing Waves, Rotating Spirals, and Turbulence," Phys. Rev. Lett. 65, 30133016 (1990).

[25] S. Nettesheim, A. von Oertzen, H. H. Rotermund, and G. Ertl, "Reaction Diffusion Patterns in the Catalytic CO-Oxidation on Pt(110): Front Propagation and Spiral Waves," J. Chem. Phys. 98, 9977-9985 (1993).

[26] A. B. Carey, R. H. Giles, Jr., and R. G. McLean, "The Landscape Epidemiology of Rabies in Virginia," Am. J. Trop. Med. Hyg. 27, 573-580 (1978).

[27] J. D. Murray, E. A. Stanley, and D. L. Brown, "On the Spatial of Rabies Among Foxes," Proc. Roy. Soc. Lond B 229, 111-150 (1986).

[28] M. Kuperman and G. Abramson, "Small World Effect in an Epidemiological Model," Phys. Rev. Lett. 86, 2909-2912 (2001). 
[29] L. S. Schulman and P. E. Seiden, "Percolation and Galaxies," Science 233, 425431 (1986).

[30] B. F. Madore and W. L. Freedman, "Self-Organizing Structures," Am. Sci. 75, 252-259 (1987).

[31] P. Parmananda, "Generalized Synchronization of Spatiotemporal Chemical Chaos," Phys. Rev. E 56, 1595-1598 (1997).

[32] S. K. Scott, Oscillations, Waves, and Chaos in Chemical Kinetics (Oxford University Press, New York, 1995).

[33] J. J. Tyson and P. C. Fife, "Target Patterns in a Realistic Model of the BelousovZhabotinskii Reaction,” J. Chem. Phys. 73, 2224-2237 (1980).

[34] H.-J. Krug, L. Pohlmann, and L. Kuhnert, "Analysis of the Modified Complete Oregonator Accounting for Oxygen Sensitivity and Photosensitivity of BelousovZhabotinsky Systems," J. Phys. Chem. 94, 4862-4866 (1990).

[35] P. De Kepper and J. Boissonade in [1], p. 223-256.

[36] C. K. McKinnon and R. J. Field, "Continuously Stirred Tank Reactor Bistability in the Belousov-Zhabotinskii Reaction: Oregonator and Explodator Models," J. Phys. Chem. 90, 166-168 (1986). 


\section{Chapter 4}

\section{Synchronization of Spatiotemporal Patterns in Locally Coupled Excitable Media}

\subsection{Introduction}

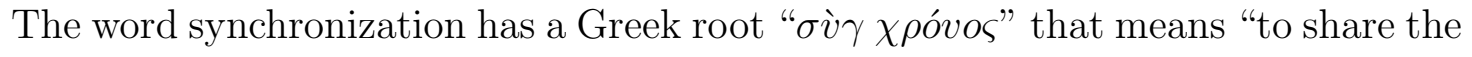
common time." This original meaning has been maintained in the colloquial use of the word synchronization, as agreement or correlation in time of different processes [1]. The study of synchronization can be dated back to 1673 when Christian Huygens, the famous Dutch mathematician, astronomer and physicist, observed synchronization of two pendulum clocks hanging from a common support. The oscillations of the two pendulums coincided perfectly and they moved always in opposite directions [2]. By the end of last century, the synchronization of periodic oscillators [3] had been developed as an applied science [4].

The discovery of synchronization of chaos occurred in the 1980s $[5,6]$; however, the beginning of systematic studies of synchronization of chaotic systems was from 1990, when Pecora and Carroll first experimentally demonstrated the synchronization of 
chaos in an electric circuit [7]. Since then, the synchronization of chaotic systems has been a very active field in nonlinear dynamics [8-11]. Now, the concept of synchronization of chaotic systems has evolved to that of complete or identical synchronization (CS or IS) [12], phase synchronization (PS) [13], lag synchronization (LS) [14], generalized synchronization (GS) [15], intermittent lag synchronization (ILS) [14], imperfect phase synchronization (IPS) [16], and almost synchronization (AS) [17]. The relationship between these different types of synchronization and the transitions between them has been studied recently in $[14,18]$. In addition to the theoretical studies, there are many experimental verifications, such as in the cardiorespiratory system [19], in the human brain [20], and in the cells of paddlefish [21]. Other experimental studies include applications in secure communications with electronic circuits [22], chaotic lasers [23], semiconductor lasers [24], and experimental control of chaos in many areas such as, e.g., chemistry [25], laser physics [26], electronic circuits [27] and mechanical systems [28].

Today, in terms of fundamental science, the basic framework underlying the synchronization of chaos in lower dimensional systems is well understood. The natural continuation of this work lies in the investigation of the synchronization of space extended systems exhibiting spatiotemporal chaos. Fluids, optics, coupled electrical oscillators, chemical reactions, plasmas, and biological systems are just a few examples of spatially extended systems that can be described by either cellular automata (CA), coupled map lattices (CMLs), coupled ordinary differential equations (CODEs), or partial differential equations (PDEs) [29]. In this Section, we review the schemes leading to synchronization and the studies on synchronization of spatiotemporal chaos.

\subsubsection{Category of schemes leading to synchronization}

Many different approaches leading to synchronization have been proposed. They fall into three schemes: coupling, external forcing, and noise. Each scheme allows a 
number of alternative realizations. Unidirectional coupling and bidirectional coupling represent two different configurations of coupling. The first type is also called drivingresponse (or master-slave) coupling. This implies that the drive system evolves freely and drives the evolution of response system by a driving function, such that external synchronization is produced. A typical example of this type of synchronization is found in studies of communication with chaos $[7,22]$. In bidirectional coupling, both systems are coupled to each other in such a way that the coupling factor induces an adjustment of the "rhythms" onto a common synchronized state, and mutual synchronization is produced. This type of synchronization is often observed in physiology, e.g., between cardiac and respiratory systems [19] or between interacting neurons [30]. Different driving functions have been proposed for unidirectional coupling, such as sensor coupling [31], stochastic coupling [32], dissipative coupling [33], sporadic coupling [34], and vectorial coupling [24]. There are also different ways to realize bidirectional coupling. The differences between the two systems can be superimposed onto each system symmetrically [35]. Another realization is adding the local values of one of the systems to corresponding points of its counterpart with an appropriate coefficient [36].

External forcing can lead one or a collection of systems to entrain to the forcing frequency or to a common frequency other than the forcing frequency, both of which are called forced synchronization [37]. This scheme is used in the synchronization of periodic, chaotic and stochastic systems, which are relevant to biological systems, such as ion channels and sensory neurons. The external forcing can be periodic [38-40] or stochastic [41].

If different systems are not coupled (or only weakly coupled) and subject to a common imposed noise signal, they can be synchronized via the noise. Facilitating synchronization is another constructive effect of noise, as in stochastic resonance [42], and the corresponding synchronization is referred to as stochastic synchronization [37]. This type of synchronization is of great relevance in biology, especially in neuroscience and ecology. It has been found that noise can enhance phase synchro- 
nization of weakly coupled chaotic electrochemical oscillators [43] as well as excitable medium modeled by a discrete network of diffusively coupled Fitzhugh-Nagumo oscillators [44]. It has been applied to biological and medical systems, for example, the noise synchrony found in the human heart-respiratory system [19] and magnetoencephalograms (MEGs) of Parkinsonian patients [20]. Recently, noise-induced synchronization has been reported in pairs of chaotic oscillators [18] and noncoupled sensory neurons [45].

\subsubsection{Review of synchronization of spatiotemporal chaos}

Chaotic motion in spatially extended dynamical systems is often referred to as space-time chaos, with decaying correlations in both space and time. Popular models of space-time chaos include cellular automata (CA), coupled map lattices (CMLs), coupled ordinary differential equations (CODEs or lattices of continuous-time oscillators), and partial differential equations (PDEs). Table 4.1 shows the characters of these four dynamical systems. Cluster and global synchronization behavior in these types of systems have been studied $[30,32]$ and reviewed $[10,11]$. Here, we review the synchronization behavior between coupled pairs in these types of systems. These systems have their own individual properties; however, they also have some universal properties that make them comparable in synchronization of spatiotemporal chaos. Kocarev et al. [33] numerically synchronized a pair of unidirectionally coupled CMLs, CODEs, and PDEs by a similar scheme in which the response system was driven at a finite number of space points at periodic time intervals by the driving system. The synchronization transition of coupled CA [46,47], CMLs, and PDEs [47,48] has also been studied. The synchronization transition is either in the universality class of "bounded KPZ" (the Kardar-Parisi-Zhang equation with a growing limiting term [48]) or "directed percolation" depending on the local non-linearities. The primary pa-

rameter indicating synchronization of spatiotemporal chaos is synchronization error, which is the local difference between the variables of the two systems, which vanishes 
Table 4.1. Characters of space-time chaos systems

\begin{tabular}{|llll|}
\hline System & Time & Space & States \\
\hline CA & Discrete & Discrete & Discrete \\
CML & Discrete & Discrete & Continuous \\
CODE & Continuous & Discrete & Continuous \\
PDE & Continuous & Continuous & Continuous \\
\hline
\end{tabular}

to zero when synchronization is achieved. When the two spatiotemporal chaotic systems are synchronized, there are no patterns $[33,46,49,50]$ or regular patterns $[10,36]$ on the space-time plot of synchronization error.

\section{Space discrete systems}

In cellular automata, not only are time and space discrete, but also the field is allowed to have only discrete values (usually one considers automata with two states "0" and "1"). Cellular automata [51] have weaker chaotic properties than coupled map lattices. There are few studies on synchronization of coupled CA, although the synchronization transition of coupled CA has been studied [46,47]. Most studies have focused on CMLs, which are idealized systems for spatiotemporal chaos. CMLs are discrete in time and space and the individual maps are usually diffusively coupled in space to nearest neighbors (local coupling) [33], to one neighbor (one-way CMLs) [52], asymmetrically to nearest neighbors (asymmetrically coupled CMLs) [53], or globally coupled with a mean-field (global coupling) [54]. Kocarev et al. [33] numerically synchronized a pair of unidirectionally coupled CMLs by driving the response system at spatially periodic points. In order to obtain higher efficiency in synchronizing spatiotemporal chaos, which may perform simultaneously multichannel secure communication and secure information storage, researchers have sought fewer linking points between the driving and the response systems in the synchronization schemes. $\mathrm{Hu}$ 
et al. [52] proposed that two identical one-dimensional and two-dimensional one-way CMLs could be synchronized by driving a single site. Jiang and Parmananda [53] reported synchronization of two asymmetrically coupled CMLs by linking them with a common signal through one-site connection.

Coupled ordinary differential equations (CODEs) are systems of ODEs with a discrete spatial structure and are also called lattice differential equations [55]. Chaos synchronization within an array of coupled CODEs has been studied extensively [34, 41], and the synchronization found in these studies was coherent motions. Kocarev and Parlitz [34] studied the synchronization of two coupled arrays of CODEs. This was the first report of synchronization between two coupled chaotic spatiotemporal systems. In their study, both drive and response systems were arrays of diffusively coupled Lorenz systems, and the coupling between the two arrays was active at discrete times only, which is called "sporadic coupling." The response system oscillated freely and independently from the drive system except for the moments when all the elements of the response system were forced to the values of the corresponding elements in the drive system. Later, Kocarev et al. [33] numerically obtained similar results by using a "dissipative coupling" scheme in which the response system was fed the information from the drive system at spatial periodic locations when coupling was introduced.

\section{Space continuous systems}

Different PDE models such as the complex Ginzburg-Landau equation (CGLE) [31, 33, 34, 36, 40, 49, 56-58], Kuramoto-Sivashinsky equation (KSE) [10], Gray-Scott model [33,34], and a model describing the CO oxidation on a singel-crystal $\mathrm{Pt}(110)$ surface [59] have been used in studies of the synchronization of spatiotemporal chaos in coupled PDEs. The CGLE $[29,60]$ describes universal dynamical features of extended systems close to a Hopf bifurcation, and it has been used to describe many different dynamical behaviors in laser physics, fluid dynamics, chemical turbulence, and bluff body wakes [49]. Depending on the range of the parameters, the CGLE displays 
different types of spatiotemporal chaos: phase turbulence (PD), defect turbulence or amplitude turbulence (DT or AT), and spatiotemporal intermittency (STI). The Gray-Scott model [61] describes an autocatalytic reaction-diffusion system that can exhibit mixed-mode spatiotemporal chaos. The Kuramoto-Sivashinsky equation [62, 63 is known to demonstrate spatiotemporal chaos if the spatial domain is sufficiently large $[10]$.

Synchronization of spatiotemporal chaos in coupled PDEs is the most challenging to study, since space and time are continuous in PDEs. Time and space are discretized in numerical simulations of PDEs, which yields "numerical" CMLs. In common CMLs, however, each space point is explicitly defined, unlike in simulation of PDEs, where a grid point represents an area of space. Hence, the point to point coupling scheme used in CMLs can not be directly applied to PDEs. One must also to take into account the characteristics of the discretization scheme and the effects of point to point coupling on the neighborhood points, as emphasized in [31]. To realize coupling schemes that have physical meaning in the context of the original system is of primary importance in studies of spatiotemporal chaos synchronization described by PDEs. Kocarev et al. $[33,50]$ introduced a general approach for synchronization pairs of unidirectionally coupled PDEs with spatiotemporal chaotic dynamics. Their scheme involved applying the driving signal only at a finite number of periodic space points at periodic time intervals. They studied synchronized pairs of PDEs described by the Gray-Scott model [33,50], the CGLE [50], and the KSE [50].

The first study on complete synchronization of spatiotemporal chaos of coupled PDEs was carried out by Amengual et al. [36]. They studied the synchronization of a pair of one-dimensional CGLEs describing STI regimes. Spatiotemporal synchronization of localized structures was achieved by introducing mutual coupling on the amplitude of the variable (i.e. coupling was added on one of the terms of the CGLE), unlike other studies, in which coupling was introduced to the field (as an additional term in the CGLE). Increasing the coupling strength, the dynamics of the coupled 
STI regimes became increasingly correlated, and finally STI disappeared and was replaced by regular patterns. Recently, Boccaletti et al. [49] and Junge and Parlitz [31] reported the control and synchronization of AT and PT modeled by CGLEs in unidirectionally coupled identical systems using "adaptive coupling," and "sensor coupling," respectively. In the former case, a finite number of controllers, which is determined by the correlation domain, are used in the infinite space and the coupling strength is adapted to the local dynamics. In the latter case, the coupling signal was averaged on a local space, and the number of coupling signals was scaled linearly with the system length.

The advantage of using the CGLE is that it allows the phase and amplitude of oscillation to be used explicitly to observe phase synchronization or complete synchronization. Boccaletti et al. [56] studied the synchronization of a pair of bidirectionally coupled nonidentical extended systems described by CGLEs with symmetric coupling in the AT and PT regimes. They studied changes in the phase differences and amplitude differences between these two systems as a function of coupling strength and found that the transition to complete synchronization or phase synchronization depended on the differences between the systems as well as the coupling strength. Junge and Parlitz [57] studied the synchronization of unidirectionally coupled nonidentical CGLEs. They have shown that the transition from frequency synchronization to phase synchronization and generalized synchronization occurs when the coupling strength is increased.

The synchronization of a pair of one-dimensional unidirectionally coupled model systems used for description of the CO oxidation on a single-crystal $\mathrm{Pt}(110)$ surface was studied by Parmananda [59]. In this study, the local difference between the drive and response systems was superimposed onto the dynamical evolution of the response system. Synchronization was achieved via this continuous feedback for both identical and nonidentical systems. The spatiotemporal chaos in the response system was suppressed, maintained, or the dynamics converged to that of the driving system, 
depending on the characteristics of the driving system.

Synchronization in extended systems is important in studies of dynamical systems. So far, most of the research in this area has been carried out on one-dimensional systems. In the next part of this Chapter, we will introduce our study of synchronization of spatiotemporal patterns between two photosensitive BZ reaction systems by local coupling. We will demonstrate synchronization behavior in experiments as well as in numerical simulations.

\subsection{Experimental Study}

\subsubsection{Preparation of stock solutions}

All the chemicals except $\mathrm{Ru}(\mathrm{bpy})_{3}{ }^{2+}$ were of analytical grade (Fisher) and used without further purification. Stock solutions of sodium bromide ( $\mathrm{NaBr})$, sodium bromate $\left(\mathrm{NaBrO}_{3}\right)$, malonic acid (MA), and sulfuric acid $\left(\mathrm{H}_{2} \mathrm{SO}_{4}\right)$ were prepared with doubly distilled water and kept in the refrigerator at a temperature of about $3{ }^{\circ} \mathrm{C}$. Initial concentrations of $\mathrm{NaBr}, \mathrm{NaBrO}_{3}$ and $\mathrm{MA}$ were $1.0 \mathrm{M}$; initial concentration of $\mathrm{H}_{2} \mathrm{SO}_{4}$ was $3.0 \mathrm{M}$.

The stock waterglass solution of $\mathrm{SiO}_{2}$ was prepared with solid sodium silicate (Fluka), which contained $60 \% \mathrm{SiO}_{2}$. The initial concentration of $15 \%(\mathrm{w} / \mathrm{w})$ sodium silicate was prepared by dissolving the appropriate amount of sodium silicate in boiling stirred doubly distilled water. It was boiled for a few minutes and kept hot while vigorously stirring for about 30 minutes until it was transparent. After cooling to room temperature, water was added to make up for evaporation and the solution was filtered.

The stock solution of $\mathrm{Ru}(\mathrm{bpy})_{3}{ }^{2+}$ was prepared by disloving the sulfate salt of $\mathrm{Ru}(\mathrm{bpy})_{3}{ }^{2+}$, which was prepared as described in Appendix A, in doubly distilled 
water. The concentration of $\mathrm{Ru}(\mathrm{bpy})_{3}{ }^{2+}$ was calculated using the extinction coefficient $13400 \mathrm{M}^{-1} \mathrm{~cm}^{-1}$ for $\mathrm{Ru}(\text { bpy })_{3}{ }^{2+}$ at its maxium absorbance $454 \mathrm{~nm}$. The absorption spectra were measured with a Hewlett-Packard (HP845x UV-Visible ChemStation) spectrophotometer.

\subsubsection{Prepare of the catalyst-free BZ solution}

The catalyst-free BZ solution was prepared freshly before each experiment and was kept in an ice bath from the time of mixing until the end of the experiment. Solutions of $\mathrm{NaBrO}_{3}, \mathrm{H}_{2} \mathrm{SO}_{4}$, and $\mathrm{MA}$ were mixed together in the given sequence in a volumetric flask. Bromomalonic acid (BrMA) was prepared in situ by dropwise

addition of a stoichiometric amount of $\mathrm{NaBr}$ to the solution according to reaction [64]

$$
\mathrm{BrO}_{3}^{-}+2 \mathrm{Br}^{-}+3 \mathrm{MA}+3 \mathrm{H}^{+}=3 \mathrm{BrMA}+3 \mathrm{H}_{2} \mathrm{O}
$$

A computer-controlled syringe pump (HARVARD 33) was used to maintain the desired rate of $1 \mathrm{ml} / \mathrm{min}$ for the addition. The solution was homogenized by vigorous mechanical stirring until both the color and the smell of bromine disappeared. The chemical composition of the reaction mixture is shown in Table 4.2, as an example for making $500 \mathrm{ml}$ of catalyst-free BZ solution from the stock solutions.

Table 4.2. Recipe of the catalyst-free BZ solution

\begin{tabular}{llll}
\hline Chemicals & Initial concentrations $(\mathrm{M})$ & Volume $(\mathrm{ml})$ & Final concentrations $(\mathrm{M})$ \\
\hline $\mathrm{NaBrO}_{3}$ & 1.0 & 167.5 & 0.28 \\
$\mathrm{MA}$ & 1.0 & 107.5 & 0.05 \\
$\mathrm{H}_{2} \mathrm{SO}_{4}$ & 3.0 & 94.2 & 0.4 \\
$\mathrm{BrMA}$ & $\mathrm{N} / \mathrm{A}$ & $\mathrm{N} / \mathrm{A}$ & 0.165 \\
$\mathrm{NaBr}$ & 1.0 & 55 & 0 \\
$\mathrm{H}_{2} \mathrm{O}$ & N/A & 75.8 & N/A \\
\hline
\end{tabular}




\subsubsection{Preparation of gel}

An appropriate amount of $15 \%(\mathrm{w} / \mathrm{w})$ sodium silicate solution, $\mathrm{Ru}(\mathrm{bpy})_{3}{ }^{2+}$ solution, and doubly distilled water were well mixed in a clean vial, and an appropriate amount of $1.2 \mathrm{M} \mathrm{H}_{2} \mathrm{SO}_{4}$ was added while shaking the solution. After mixing the solution vigorously for $2-3$ seconds, $0.9 \mathrm{ml}$ of the mixture was rapidly removed and injected under a glass slide which was supported by two pieces of $0.3 \mathrm{~mm}$ lead on a piece of Plexiglas, where it was allowed to cure. The gel with the glass was removed from the Plexiglas and trimmed to the designed size. The gel is transparent, without any inhomogeneities visible to the naked eye. This preparation of silica gel is an efficient and convenient procedure. The amounts of water and $\mathrm{H}_{2} \mathrm{SO}_{4}$ control the gelation rate, and the amounts of $\mathrm{Na}_{2} \mathrm{SiO}_{3}$ solution and $\mathrm{Ru}(\text { bpy })_{3}{ }^{2+}$ correspond to their concentrations in the experiment. In our study, we used $10 \%$ (w/w) $\mathrm{Na}_{2} \mathrm{SiO}_{3}$ and $2.0 \mathrm{mM} \mathrm{Ru}(\mathrm{bpy})_{3}{ }^{2+}$ and cast a uniform gel with dimensions of $0.3 \mathrm{~mm} \times 20.0$ $\mathrm{mm} \times 25.6 \mathrm{~mm}$.

\subsubsection{Experimental setup}

The experimental setup is shown as Fig. 4.1. It is composed of two parts: one is where the BZ reaction takes place; the other is the instrumentation to control and record the spatiotemporal patterns in the photosensitive BZ reaction.

The microscope slide bearing the gel, in which the catalyst is immobilized, is placed faceup in a thermostatic reactor and covered by the catalyst-free BZ solution, which is continuously fed to maintain constant, nonequilibrium conditions [65]. In gel systems $[66,67]$, there are no hydrodynamic effects that perturb the spatial pattern, which is essential for the systematic study of chemical waves.

The catalyst-free BZ solution, matintained in an ice bath, was pumped continuously at a flow rate of $2.0 \mathrm{ml} / \mathrm{min}$ using a peristaltic pump (Lsmatec) to the reactor 


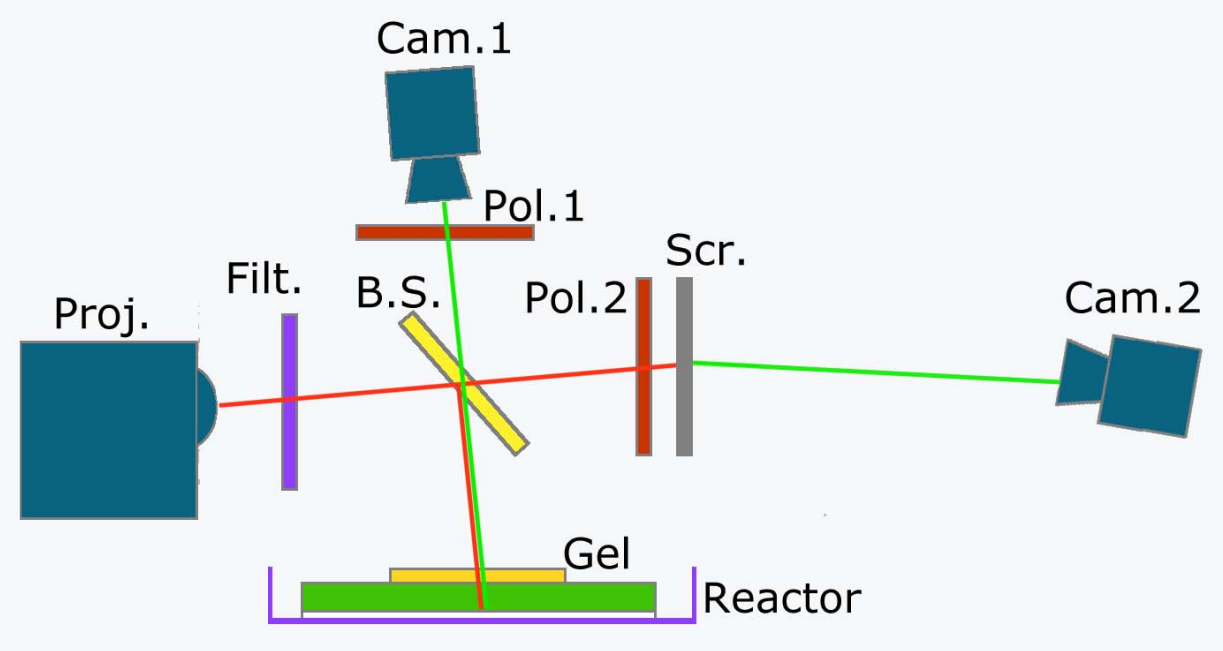

Figure 4.1. Experimental setup. See text for explanation of each unit. 
through a degaser (Uniflow Degasys Ultimate DV 4010). The reaction mixture was also pumped out at the same rate such that the thickness of the layer of solution in the reactor was kept constant during the experiment.

An illumination profile produced by a modified video projector (Proj. in Fig. 4.1), which is controlled by a computer, is projected onto the gel medium through a 460 nm bandpass filter (Filt. in Fig. 4.1) and a half mirror beam splitter (B.S. in Fig. 4.1). The light from the projector is scattered from a white membrane filter (Pall Gelman Laboratory, Super-450, $0.45 \mu \mathrm{m})$ positioned between the glass slide and the bottom of the reactor, and serves as background for observing the chemical patterns. A charge coupled device (CCD) video camera, the primary camera (Cam. 1 in Fig. 4.1), fitted with a macro lens is used to record the images of the chemical patterns, which are analyzed in real time by the computer for performing the feedback profile. Using another CCD video camera, the reference camera (Cam. 2 in Fig. 4.1), adjustments are made to the projected image until the actual projected pattern, received by both the gel and the reference camera, matches the desired distribution before each experiment. The illumination pattern is established by communication between the projector and the reference camera such that the inherent spatial non-uniformity and temporal instability of the projector response are reduced to a minimum level.

The beam splitter combines the illumination and observation optical paths by matching the illumination and observation angles. The beam from the video projector is split into two: the reflected beam travels down to the gel, while the beam passing through the beam splitter forms a reference image on the diffusive screen (Scr. in Fig. 4.1). To reduce the amount of light reflected from the reference screen to the primary video camera, a pair of crossed polarizers (Pol. 1 and Pol. 2 in Fig. 4.1) are placed as indicted in Fig. 4.1. Distances along the optical axis from the beam splitter to the gel and to the reference screen are equal (red lines shown in Fig. 4.1), producing the same size images. The primary video camera is located above the beam splitter such that its optical axis coincides with the path of the light between the beam splitter 
and the gel. The reference camera is located at the same distance from the reference screen as the primary camera from the gel (green lines shown in Fig. 4.1); thus the projected images are received with the same scale by both cameras. Most of the plane optical surfaces in the system are tilted by a small angle from perpendicular to avoid reflections from the optical path, as shown in Fig. 4.1.

When $\mathrm{Ru}(\mathrm{bpy})_{3}{ }^{2+}$ is oxidized to $\mathrm{Ru}(\mathrm{bpy})_{3}{ }^{3+}$, its color changes from orange-red to blue-green. The oxidized state, $\mathrm{Ru}(\mathrm{bpy})_{3}{ }^{3+}$, absorbs much less light than the reduced state. The illumination beam passes through the gel to the white membrane filter, scatters, and travels back, to be observed by the primary camera. Since the distance between the gel and the diffuser is relatively short (the thickness of the microscope slide is $1.0 \mathrm{~mm}$ ), the profile of the wave in the gel may be seen as a "shadow" on the membrane, as shown in Fig. 4.2. If the observation angle of the camera is chosen to be the same as the angle of incidence of the illuminating beam, the interference from the "shadow" is avoided, and the optical path through the gel is effectively doubled, thus doubling the contrast of the observed pattern. This is possible because the divergence of the illuminating beam over the distance between the gel and the diffuser is negligible, and thus the "shadows" are faithful images of the chemical waves. The lower panel of Fig. 4.2 shows the observations for three different observing angles, where $\mathrm{B}$ is the one we used in our experiments. If the observation angle is $\mathrm{A}$ or $\mathrm{C}$, the image and its profile ("shadow") will partially overlap, as shown in the lower panel of Fig. 4.2. The primary camera is mounted at such an angle that the observation angle of the camera is the same as the angle of incidence of the illuminating beam, and the reference camera is also mounted at an appropriate angle.

The gray scale between 0 and 255 was calibrated to the illumination intensity in $\mathrm{mW} / \mathrm{cm}^{2}$ as shown in Fig. 4.3. Through an iterative algorithm involving communication between the projector and the reference camera, the gray level of the reference camera matched the desired gray level in the experiment very well, as shown in Fig. 4.3. This was accomplished by adjusting the grey level of the projector with the set- 


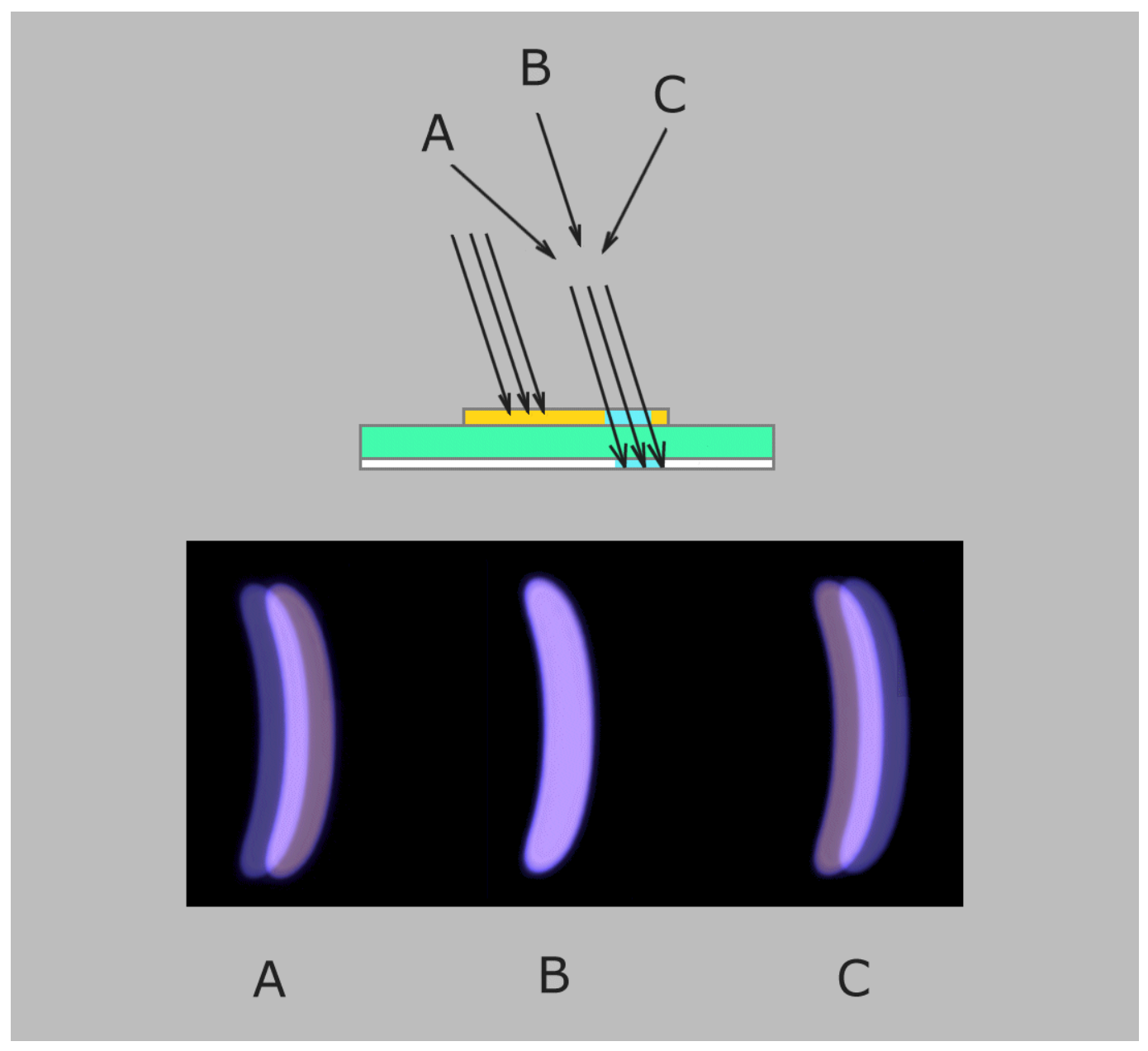

Figure 4.2. Observation angles and the effects. The three lines A, B, C above represent three possible observation angles, and the two sets of parallel lines represent the incident light transitted through gel and glass slide to the white membrane filter. The purple crescentshaped curves in the lower panel represent the chemical waves and their "shadows." 


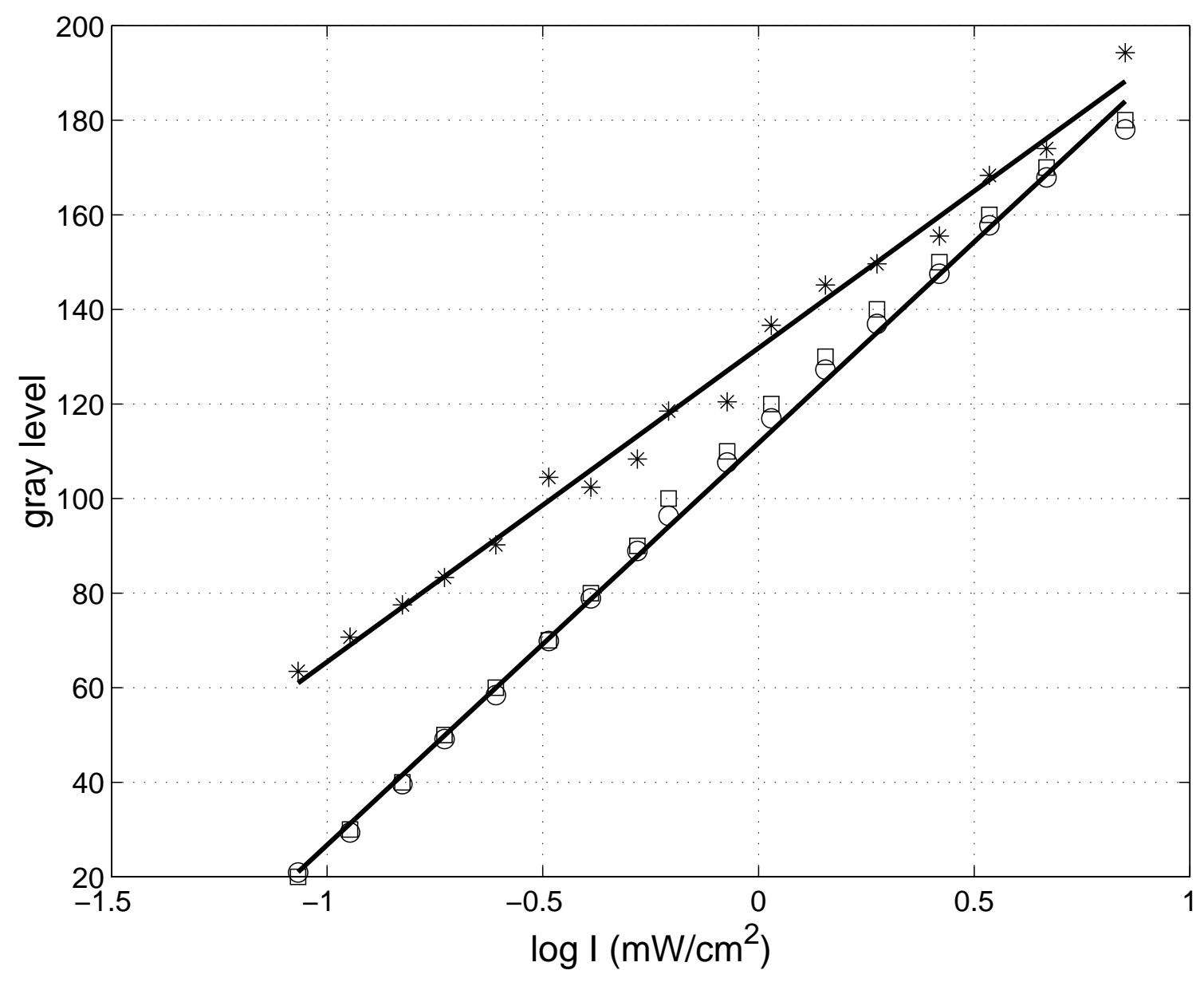

Figure 4.3. The relationship between light intensity $I$ in $\mathrm{mW} / \mathrm{cm}^{2}$ and the gray levels of the reference camera (circle), the projector (star), and the desired values (square) in an experiment. Fitting lines reveal the linear relationship between the gray level and the common logarithm of the light intensity. 
tings of the reference camera shown in Tabel 4.3. Data analysis revealed a linear relationship between the gray level and the common logarithm of the light intensity.

Table 4.3. Setting of the reference camera

\begin{tabular}{llll}
\hline Brightness & Contrast & Iris & Gamma \\
\hline 210 & 160 & between 3.5 and 5.6 & 0.45 \\
\hline
\end{tabular}

\subsubsection{Coupling scheme}

We have used the photosensitive Belousov-Zhabotinsky (BZ) reaction [68], which is particularly convenient for studies on influencing existing patterns or generating new ones by the application of various types of external forcing [69-72], or by local [73], nonlocal [74], or global [75] feedback mechanisms.

The nature of the coupling is a distinctive feature of any coupled system. Mass exchange is the most commonly employed form of coupling in chemical oscillators $[76,77]$. Another type of coupling has been accomplished electrically [78], via the connection of electrodes in two continuous flow stirred tank reactors (CSTRs). Diffusive coupling of chemical spatiotemporal patterns across a membrane in a spatially extended system leading to identical synchronization of chemical wave patterns has been observed in a BZ system [79]. Here, we study two domains of excitable media that are locally coupled to each other by means of a video camera/projector through a coupling algorithm, which leads to weaker than identical synchronization.

Prior to each experiment, the projected image was adjusted at each pixel by an iterative algorithm to ensure a spatially uniform illumination field, upon which all subsequent projected images were based. The uniform illumination field is the background intensity. The local concentration of oxidized catalyst was recorded with the CCD camera, and the recorded image was divided into an array of square cells. In all experiments, the lateral size of each cell was much smaller than the spiral wave 
length. The corresponding cells in the two regions were locally coupled by an illumination field projected onto the gel medium. Bromide ions are locally produced in a photochemical cycle, and, as a result, the local excitability is appropriately modified [80].

Figure 4.4 shows an example of an image and the corresponding mutual coupling image projected onto the gel medium in the experiment. In the top panel, the spiral waves represent elevated concentrations of the oxidized catalyst, $\mathrm{Ru}(\mathrm{bpy})_{3}{ }^{3+}$, in the photosensitive BZ reaction. The medium was partitioned into two square regions, separated and surrounded by an unexcitable boundary generated with high-intensity light, as shown in the bottom panel of Fig. 4.4. The mutual coupling image is shown in the bottom panel, which was calculated from the original image in the top panel as explained in Fig. 4.5.

In Step I of Fig. 4.5, the concentration of oxidized catalyst is recorded as an image in each system (the patterns represent chemical waves in the two systems). In Step II, the two images are subtracted. If the difference is zero, the illumination is set to the background intensity (grey area) in Step III; when the difference is positive, the system is illuminated with higher intensity (white area); when it is negative, the light intensity is lowered with respect to the background (black area).

The intensity $I_{i}(\mathbf{r}, t)(i=1,2)$ of the projected illumination field was computed as described above according to the following scheme:

$$
I_{i}(\mathbf{r}, t)=I_{0} \Theta_{i} H\left(\Theta_{i}\right)
$$

where $I_{0}=7.84 \mathrm{~mW} / \mathrm{cm}^{2}$ is the background light intensity, $H(z)$ is the step function (with $H(z)=1$ for $z \geq 0$ and $H(z)=0$ for $z<0$ ), and the functions $\Theta_{i}$ are defined as

$$
\Theta_{1,2}(\mathbf{r}, t)=1 \pm \kappa\left(v_{1}(\mathbf{r}, t)-v_{2}(\mathbf{r}, t)\right)
$$

where the plus and the minus signs refer to the first and second system, respectively, 

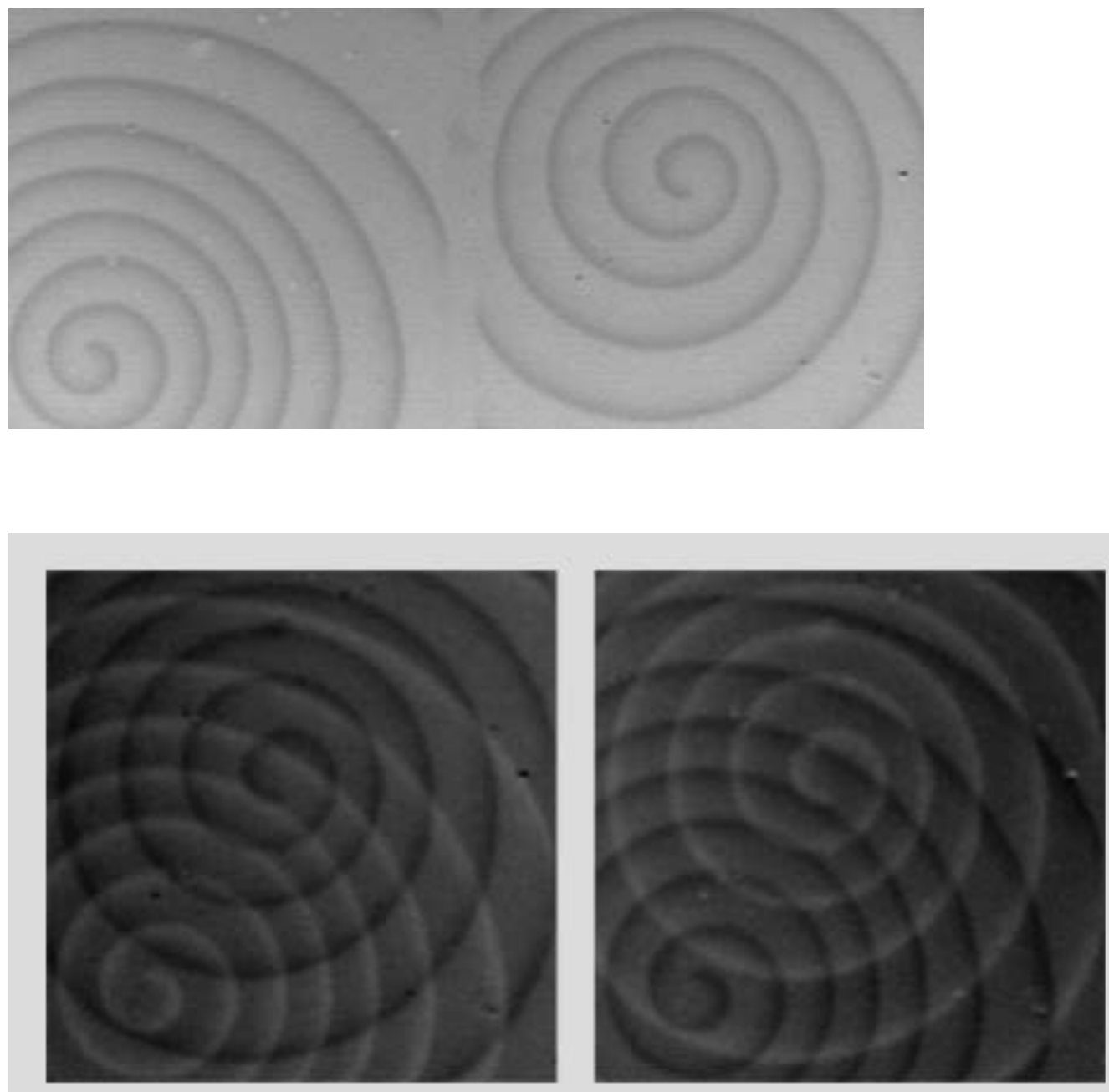

Figure 4.4. Typical example of an image and the mutual coupling image. Top: image obtained from the primary CCD camera. The total illumination field $\left(13.69 \times 6.27 \mathrm{~cm}^{2}\right)$ was divided into an array of $388 \times 178$ square cells. Bottom: mutual coupling image with boundary projected onto the chemical medium, coupling constant $\kappa=1.0$. 


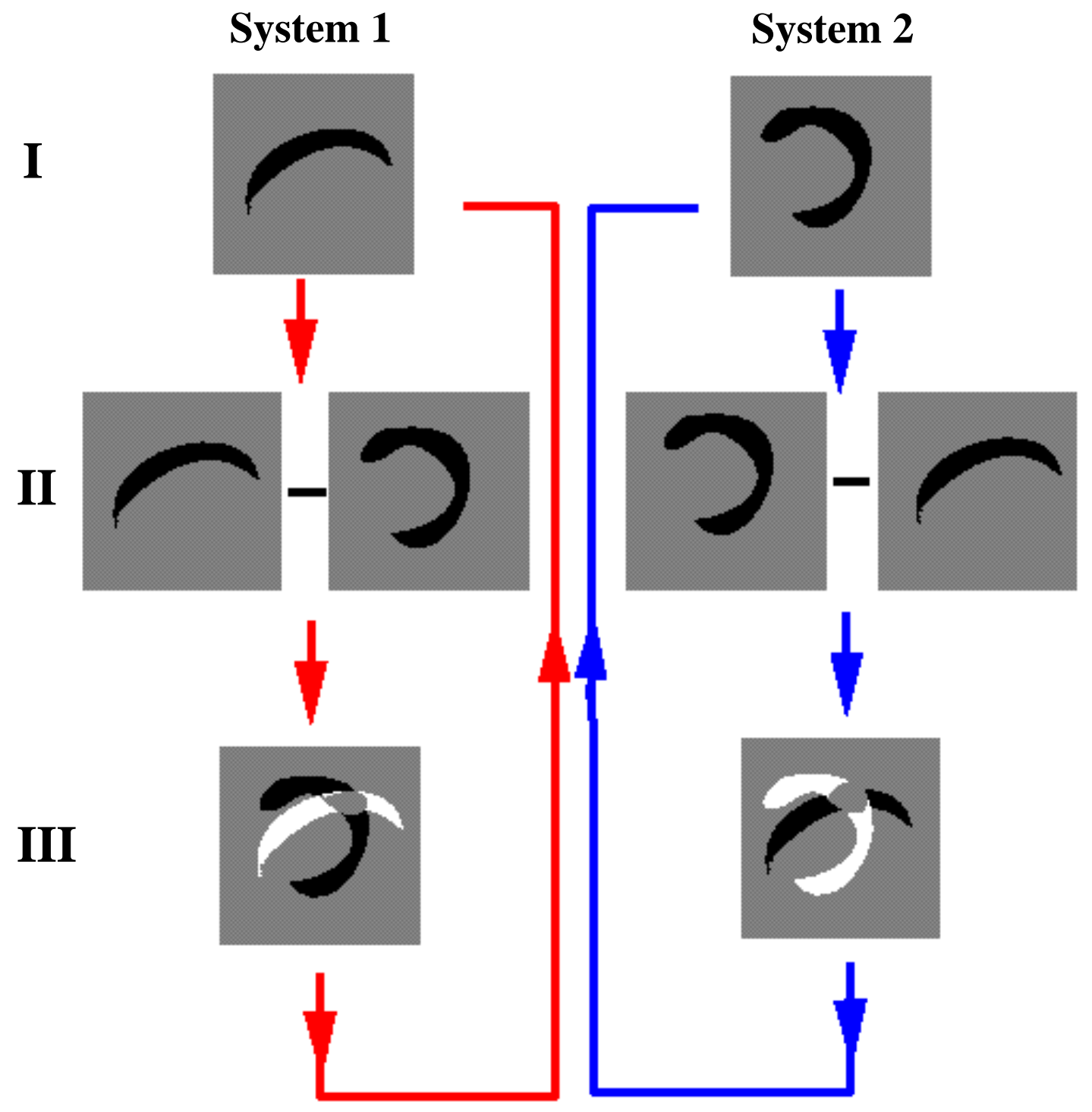

Figure 4.5. Schematic diagram of coupling scheme 
$v_{i}(\mathbf{r}, t)$ is the local concentration of the oxidized catalyst in the i-th system, and $\kappa$ specifies the strength of the symmetric mutual coupling.

\subsubsection{Experimental results I: Relaxation of disordered pat- tern to a synchronization state}

Experiments were carried out with initial conditions consisting of a random configuration of spirals [Fig. 4.6 (a)], generated by an algorithm for light-mediated spatiotemporal noise [81]. In the absence of coupling, the number of spirals in both

systems did not change over the course of the experiment. The number continuously decreased, however, when the corresponding elements in the two domains were locally coupled according to Eq. (4.2). In the first stage of the evolution, spirals were removed from each zone by collisions with counter-rotating spirals or with the boundaries of the system [Figs. 4.6 (b),(c)] until only a few spirals remained [Fig. 4.6 (d),(e)]. The coupled system eventually relaxed to one of three possible final states. The most common outcome was a single spiral in each zone, with both spirals rotating in the same direction [Fig. 4.6 (f)]. Two other final states were occasionally observed, with one zone containing a single spiral and the other in the uniform steady state or with both zones in the uniform steady state.

The robustness of synchronization behavior has been confirmed by carrying out experiments in the presence of spatiotemporal structured noise. Figure 4.7 shows a typical example of the relaxation of disordered patterns to a synchronized state through mutual coupling in the presence of colored noise. The spatiotemporal colored noise was imposed on the mutual coupling when the experiment started and was updated with each coupling image. Similar synchronizarion behavior was observed over a range of values of correlation time and noise dispersion. 


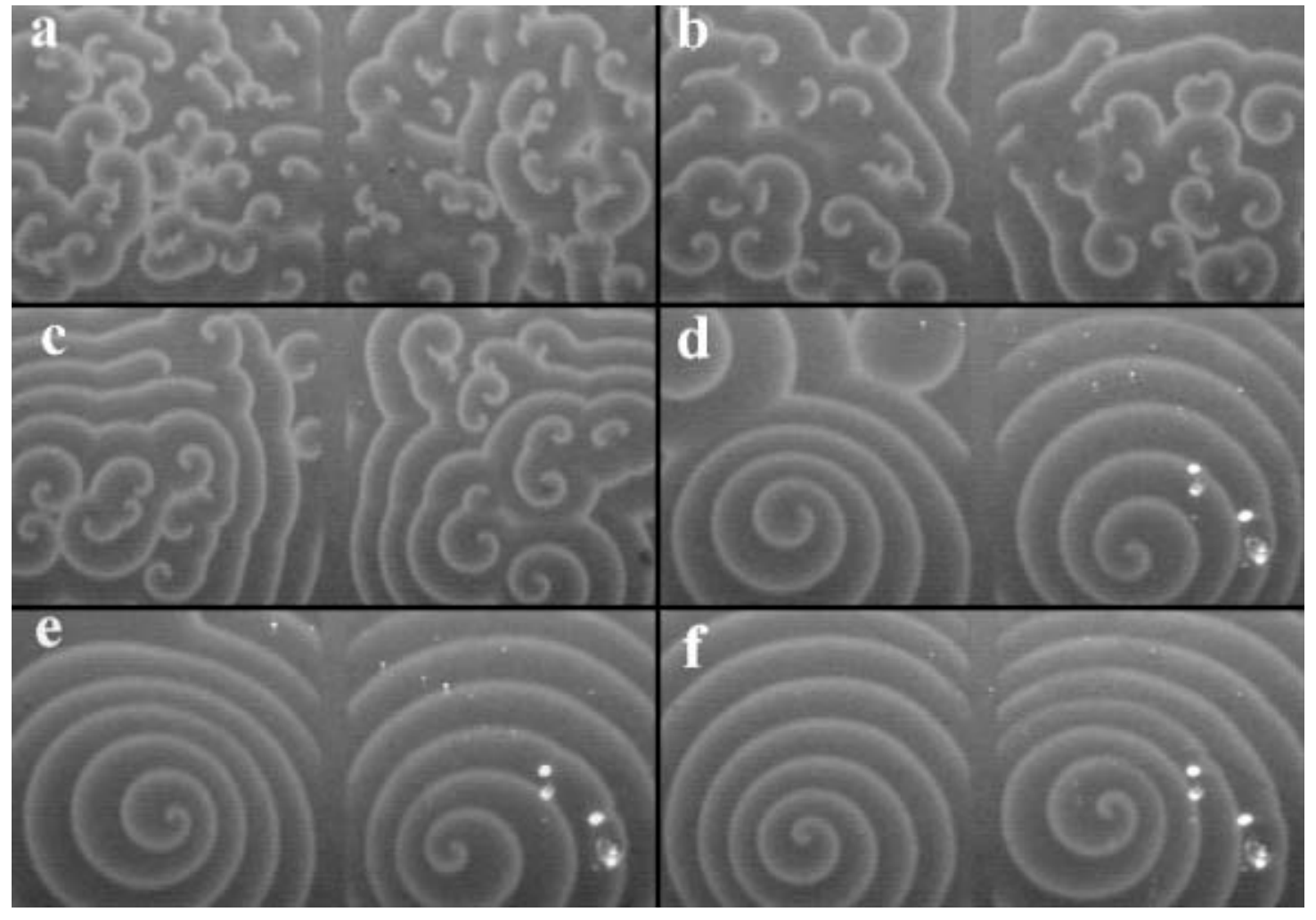

Figure 4.6. Relaxation of an initially disordered array of spirals to a synchronized state in two locally coupled excitable media. Experimental behavior of locally coupled BZ systems with $I_{0}=7.84 \mathrm{~mW} / \mathrm{cm}^{2}, T=6.5^{\circ} \mathrm{C}$, and $\kappa=0.31$. Snapshots show the distribution of $\mathrm{Ru}(\mathrm{bpy})_{3}^{3+}$ (in gray scale increasing from black to white) at $t=2.3 \mathrm{~min}(\mathrm{a}), 12.25 \mathrm{~min}(\mathrm{~b})$, $30.00 \mathrm{~min}$ (c), $67.18 \mathrm{~min}$ (d), $69.87 \mathrm{~min}(\mathrm{e})$, and $76.52 \mathrm{~min}$ (f) after starting the experiment. The total illumination field $\left(7.59 \times 3.51 \mathrm{~cm}^{2}\right)$ was divided into an array of $362 \times 167$ square cells and updated every $2.0 \mathrm{~s}$. White spots in (d), (e), and (f) are bubbles in the reaction mixture. 


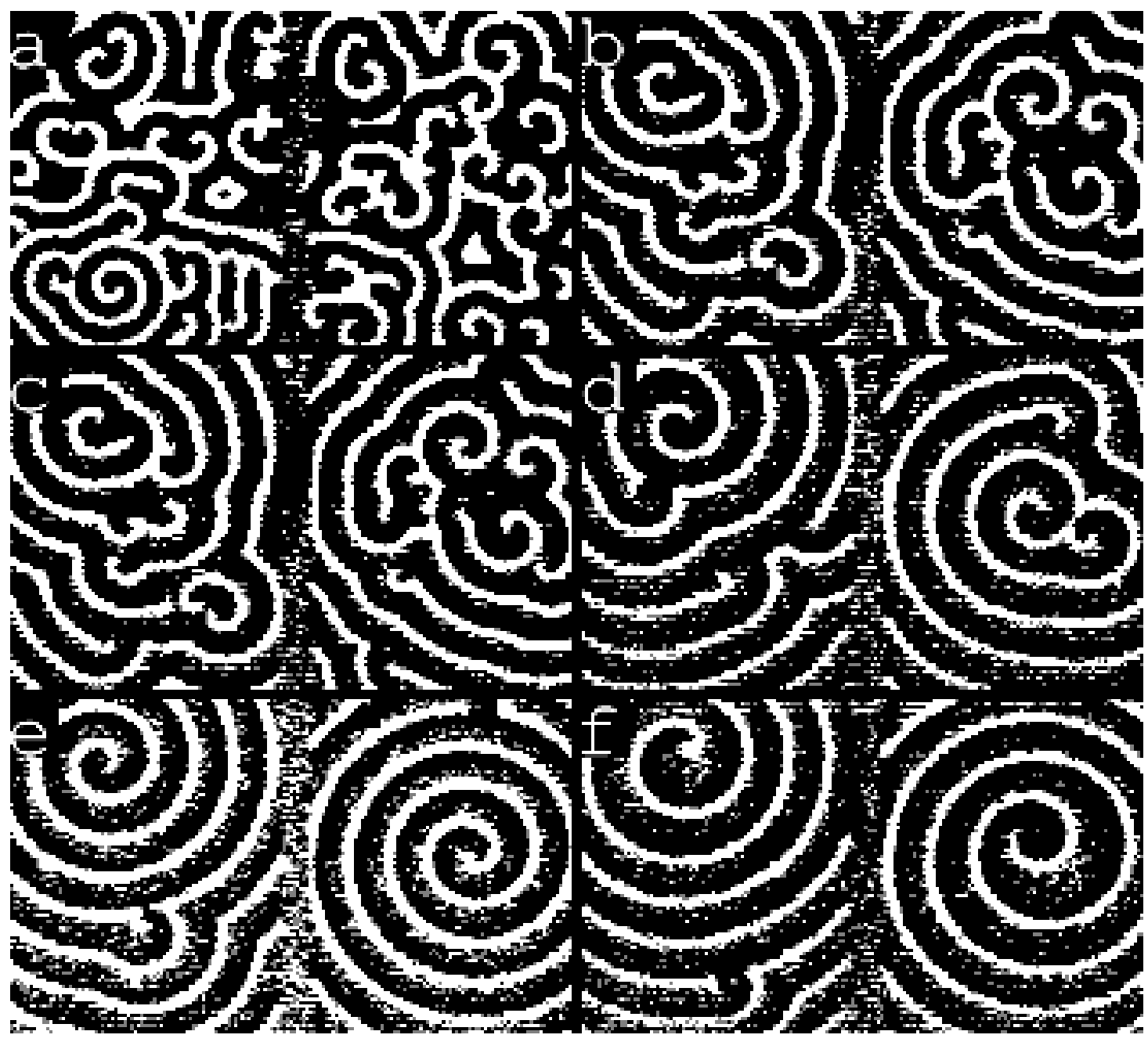

Figure 4.7. Relaxation of an initially disordered array of spirals to a synchronized state in two locally coupled excitable media in the presence of colored noise. Experimental behavior of locally coupled BZ systems with $I_{0}=7.84 \mathrm{~mW} / \mathrm{cm}^{2}, T=17.9^{\circ} \mathrm{C}$, and $\kappa=0.5$. Snapshots show the distribution of $\mathrm{Ru}(\mathrm{bpy})_{3}^{3+}$ (in gray scale increasing from black to white) at $t=0.6 \mathrm{~min}$ (a), $94.0 \mathrm{~min}$ (b), $170.0 \mathrm{~min}$ (c), $240.0 \mathrm{~min}$ (d), $282.0 \mathrm{~min}$ (e), and 328.0 min (f) after starting the experiment. The total illumination field $\left(7.57 \times 4.52 \mathrm{~cm}^{2}\right)$ was divided into an array of $173 \times 103$ square cells and updated every $5 \mathrm{~s}$. The parameters for the spatiotemporal colored noise: cluster of noise $=5 \times 5$ pixels, correlation time $=30.0 \mathrm{~s}$, dispersion $=40.0 \mathrm{~s}$. 


\subsubsection{Experimental results II: Spatiotemporal synchroniza- tion of spirals}

In this section, we report experimental results on the synchronization of two single fully developed spirals in each of the systems as shown in the top panel of Fig. 4.4. A spiral is created by a block of high intensity light that breaks the front of a propagating planar wave bound to the boundary in each domain. The free end generated evolves into a single, unperturbed spiral wave in the homogeneous background. The initial distance of the tips of the two spirals is controlled by the inhibiting blocks of high intensity light in the two domains. However, the initial distance can be only approximately controlled because of meandering of the spirals. The sense of rotating is controlled in such a way that the spirals are co-rotating if both planer waves are broken from left (or right) sides, and they are counter-rotating if they are broken from different sides. After the two spiral waves are fully developed and the medium has relaxed to the background setting, the mutual coupling is switched on. The behavior of the coupled spirals strongly depends on the initial distance $\mathrm{d}_{i}$ between the positions of the tips of the spirals. Moreover, the behavior is highly dependent on whether the two spirals rotate in the same direction or in opposite directions.

The tip position was checked manually in images collected during the experiment. The tip of the spiral was marked by the center of a cross cursor when they were matched. The coordinate of the tip of the spiral was defined in units of pixels. Figure 4.8 displays an example of two counter-rotating spirals, with $\mathrm{d}_{i}=53$ pixels. The tips of the spirals drift in the same direction. The tip distance remained almost constant for counter-rotating spirals, while it oscillated for co-rotating spirals, shown in Figs. 4.9 and 4.10. For the two co-rotating spirals, the tip motion of each spiral is generally circular with superimposed meandering. The size of the circular depends on the initial distance of the two tips and the coupling strength. 

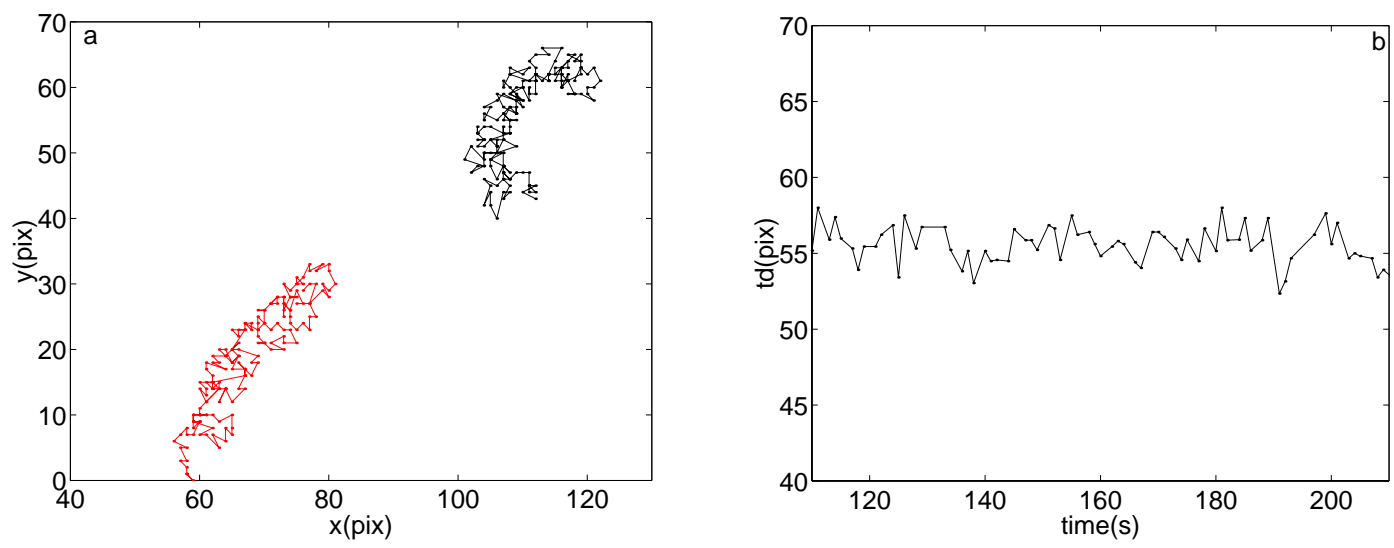

Figure 4.8. BZ experiment with two single spirals rotating in opposite directions, one in each of the two locally coupled zones, with $I_{0}=7.84 \mathrm{~mW} / \mathrm{cm}^{2}, T=10.0^{\circ} \mathrm{C}, \kappa=1.0$, and the initial spiral distance $d_{i}=53$ pixels. The total illumination field $\left(7.62 \times 3.68 \mathrm{~cm}^{2}\right)$ was divided into an array of $363 \times 175$ square cells and updated every 2 s. (a) Trajectories of the counter-rotating spiral tips in the left domain (black) and right domain (red) for $\mathrm{T}=$ $274.0 \mathrm{~s}$, displayed in superimposed coordinate systems. (b) Time series of the distance $d t$ between the two spiral tips in (a). 

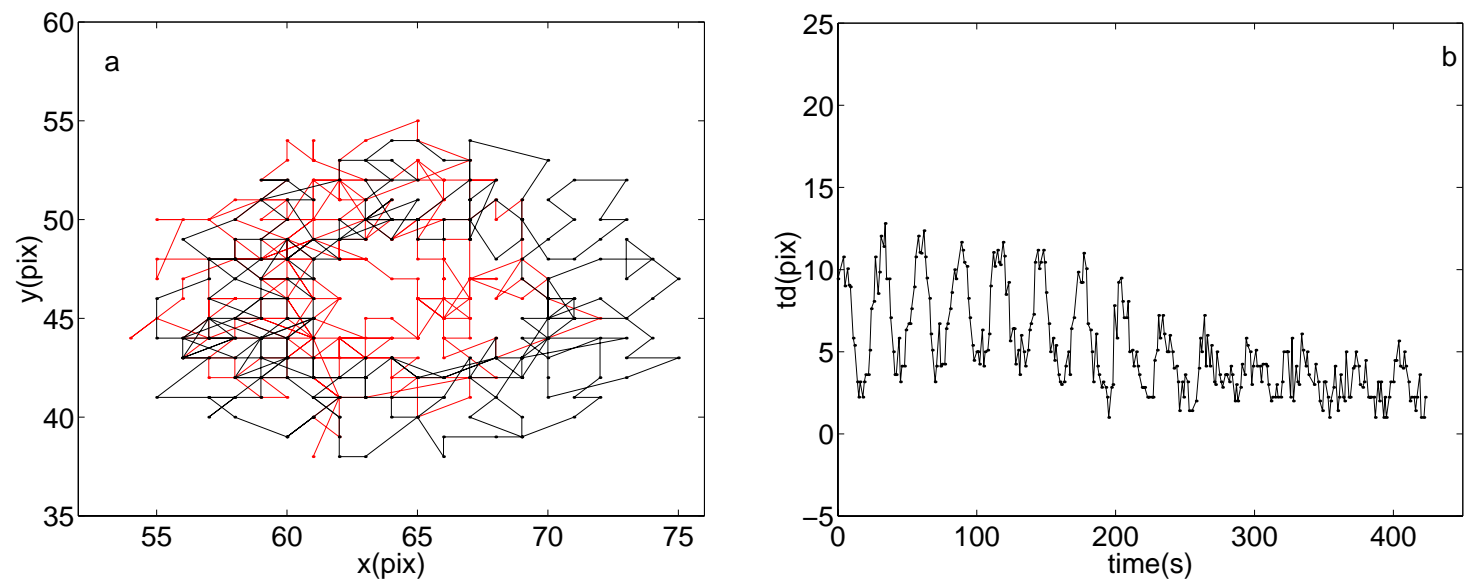

Figure 4.9. BZ experiment with single spirals rotating in same direction, one in each of the two locally coupled zones, with $I_{0}=7.84 \mathrm{~mW} / \mathrm{cm}^{2}, T=12.0^{\circ} \mathrm{C}, \kappa=1.0$, and the initial spiral distance $d_{i}=9$ pixels. The total illumination field $\left(7.62 \times 3.68 \mathrm{~cm}^{2}\right)$ was divided into an array of $357 \times 173$ square cells and updated every 2 s. (a) Trajectories of the co-rotating spiral tips in the left domain (black) and right domain (red) for $\mathrm{T}=423.0$ $\mathrm{s}$, displayed in superimposed coordinate systems. (b) Time series of the distance $d t$ between the two spiral tips in (a). 

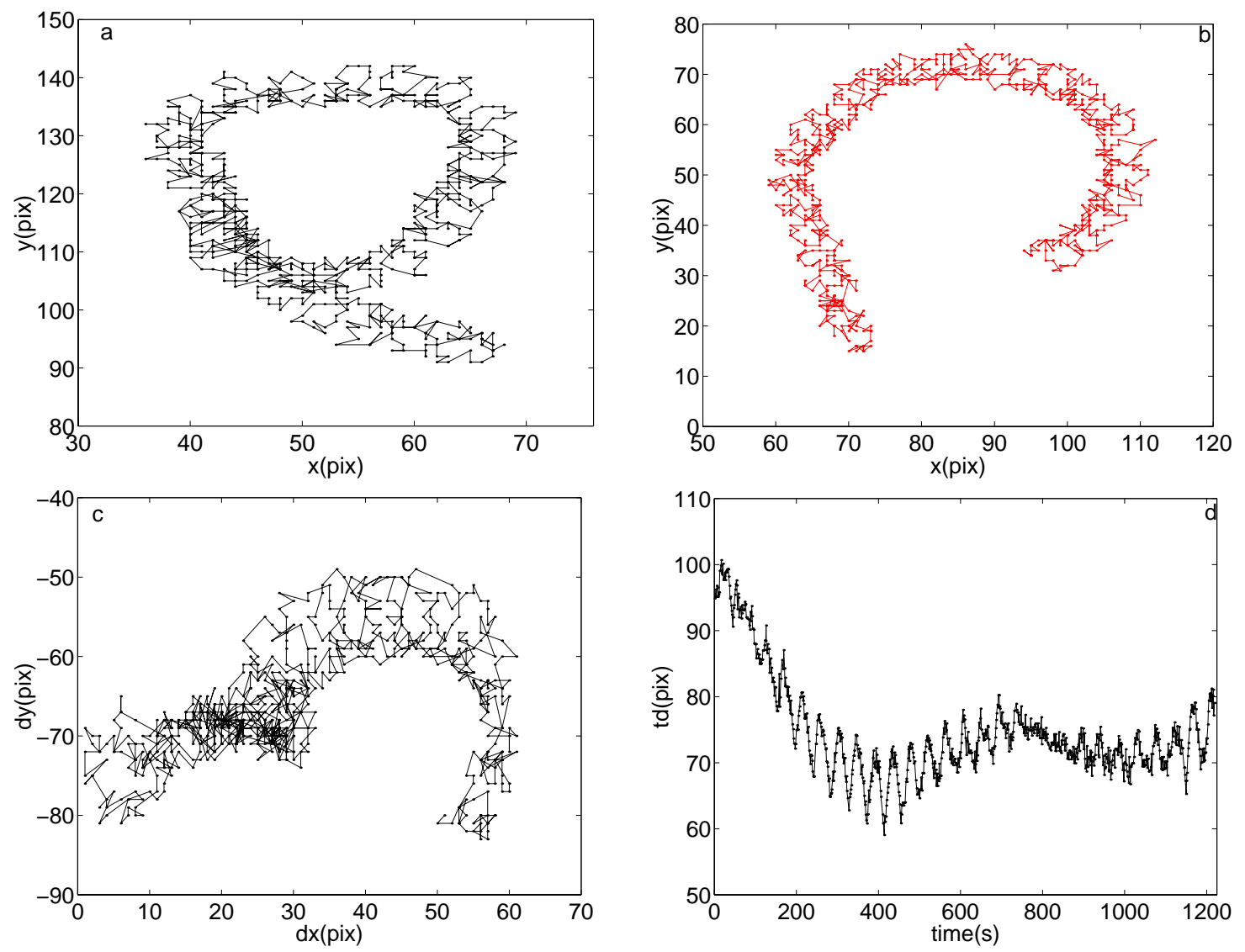

Figure 4.10. BZ experiment with single spirals rotating in same direction, one in each of the two locally coupled zones, with $I_{0}=7.84 \mathrm{~mW} / \mathrm{cm}^{2}, T=12.0^{\circ} \mathrm{C}, \kappa=0.31$, and the initial spiral distance $d_{i}=95$ pixels. The total illumination field $\left(7.62 \times 3.68 \mathrm{~cm}^{2}\right)$ was divided into an array of $357 \times 173$ square cells and updated every 2 s. (a), (b) Trajectories of the counter-rotating spiral tips in the left domain (a) and right domain (b) for $\mathrm{T}=$ $1219.0 \mathrm{~s}$, displayed in superimposed coordinate systems. (c) Trajectory of the difference vector between the two tip positions. (d) Time series of the distance $d t$ between the two spiral tips in (a) and (b). 


\subsection{Numerical Studies}

\subsubsection{Numerical model}

The experimental results shown in Fig. 4.6 (a)-(f) can be qualitatively reproduced

by numerical simulations of two locally coupled Oregonator models [70,71]. The model includes the additional terms $\phi_{i}(\mathbf{r}, t)(i=1,2)$, taking into account the photochemical bromide production [72,80]:

$$
\begin{aligned}
& \frac{\partial u_{1}}{\partial t}=\nabla^{2} u_{1}+\frac{1}{\varepsilon}\left\{u_{1}-u_{1}^{2}-\left[f v_{1}+\phi_{1}(\mathbf{r}, t)\right] \frac{u_{1}-q}{u_{1}+q}\right\}, \\
& \frac{\partial v_{1}}{\partial t}=u_{1}-v_{1}, \\
& \frac{\partial u_{2}}{\partial t}=\nabla^{2} u_{2}+\frac{1}{\varepsilon}\left\{u_{2}-u_{2}^{2}-\left[f v_{2}+\phi_{2}(\mathbf{r}, t)\right] \frac{u_{2}-q}{u_{2}+q}\right\}, \\
& \frac{\partial v_{2}}{\partial t}=u_{2}-v_{2},
\end{aligned}
$$

where the variables $u_{i}$ and $v_{i}$ correspond to the concentrations in the i-th system of the autocatalytic species $\mathrm{HBrO}_{2}$ and the oxidized catalyst; $\varepsilon$ and $q$ are scaling parameters, and $f$ is an adjustable stoichiometry parameter. The rate of bromide production from irradiation, $\phi_{i}(\mathbf{r}, t)$, is proportional to the light intensity, $I_{i}(\mathbf{r}, t)$, as given by Eq. (4.2) (with the proportionality factor $\phi_{0} / I_{0}$ ). The dynamic characteristics of the system have been analyzed in Chapter 3. The system was excitable for $\kappa=0$.

We have also performed numerical simulations of coupled Barkley models [85] 
displaying spiral behavior:

$$
\begin{aligned}
& \frac{\partial u_{1}}{\partial t}=\nabla^{2} u_{1}+\frac{1}{\varepsilon}\left(u_{1}-u_{1}^{2}\right)\left[u_{1}-\frac{v_{1}+b+\kappa\left(v_{1}-v_{2}\right)}{a}\right], \\
& \frac{\partial v_{1}}{\partial t}=u_{1}-v_{1}, \\
& \frac{\partial u_{2}}{\partial t}=\nabla^{2} u_{2}+\frac{1}{\varepsilon}\left(u_{2}-u_{2}^{2}\right)\left[u_{2}-\frac{v_{2}+b+\kappa\left(v_{2}-v_{1}\right)}{a}\right], \\
& \frac{\partial v_{2}}{\partial t}=u_{2}-v_{2},
\end{aligned}
$$

where $a$ is a scaling parameter, $\varepsilon$ is the ratio of the time scales of the activator and the inhibitor, $\kappa$ is the coupling strength, and $b$ determines the excitability threshold. The coupling was introduced much the same as in Eqs. 4.4.

\subsubsection{Numerical simulations I: Relaxation of disordered pat- tern to a synchronization state}

Figure 4.11 shows the numerical simulations of Eqs. (4.4) for the relaxation of disordered pattern to a synchronization state, which qualitatively reproduces the experimental results shown in Fig. 4.6.

\subsubsection{Numerical simulations II: Spatiotemporal synchroniza- tion of spirals}

Insights into the complex relaxation behavior shown in Fig. 4.6 can be gleaned from systematic numerical simulations with a single spiral in each system. The synchronization behavior depends on the kinetic parameters, the coupling strength, the

relative sense of rotation of the two spirals, and their initial separation distance $d_{i}$. 

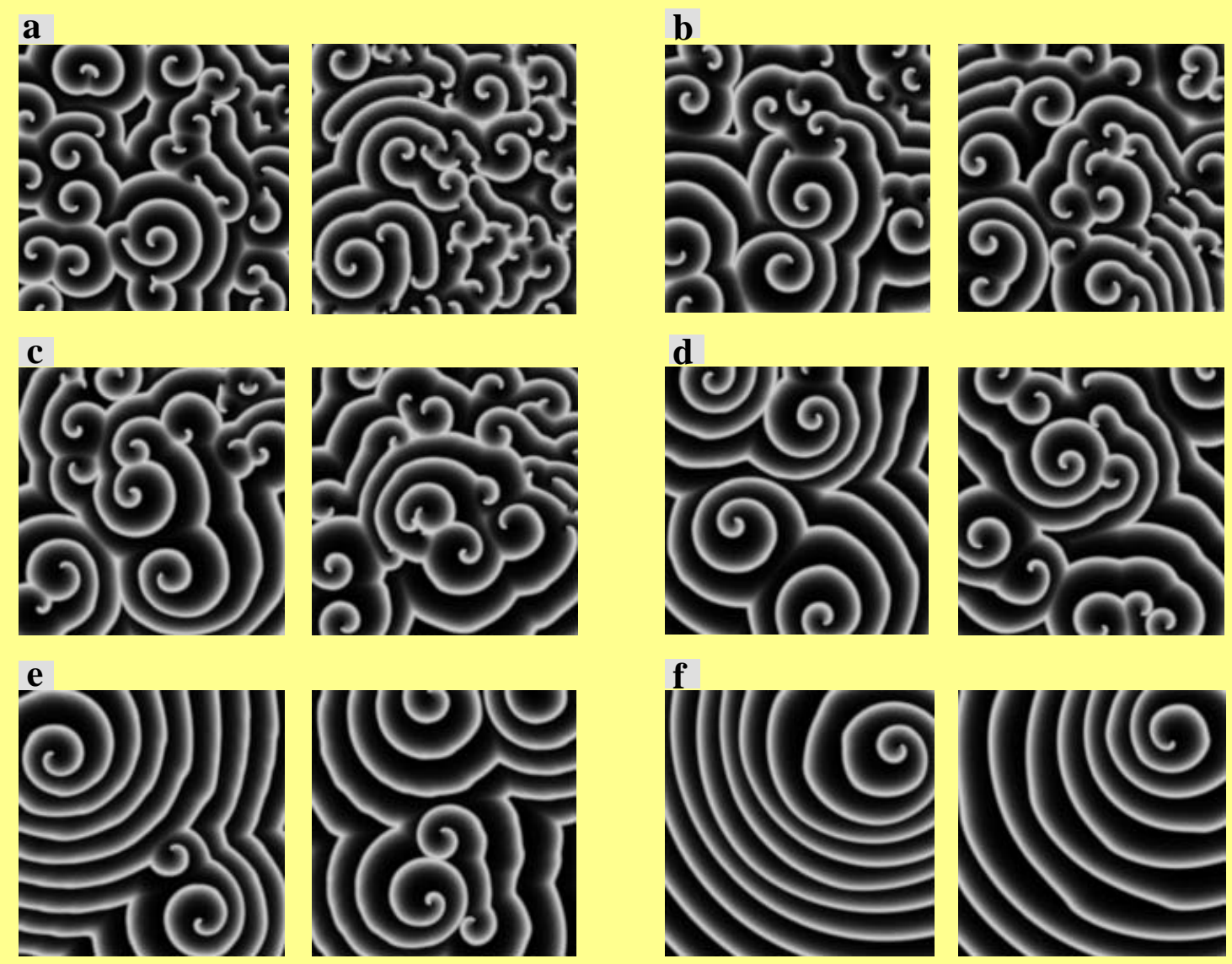

Figure 4.11. Numerical simulations of relaxation of an initially disordered array of spirals to a synchronized state in two locally coupled excitable media. Numerical simulations of Eqs. (4.4) with $\phi_{0}=0.035, \kappa=1, \varepsilon=0.03, f=1$, and $q=0.0015$ in a system of size $L=100$. The left and right panels of each snapshot show $v_{1}(x, y, t)$ and $v_{2}(x, y, t)$ in gray scale increasing from black to white. The snapshots (a)-(f) were obtained at $t=91.9$ (b), 183.7 (c), 428.7 (d), 612.5 (e), and 918.7 (f) after starting the simulation with the distribution shown in (a). 
When the two spirals rotate in opposite directions, the coupling leads to a parallel drift of the spiral tips until they are annihilated by a collision with the no-flux boundary of the system (see Fig. 4.12). This behavior was observed for values of the coupling strength $\kappa$ below 2.9 and as low as $\kappa=5 \times 10^{-3}$ (with the parameter values as in Fig. 4.11). For stronger coupling, the spirals were destroyed by breakup and both systems relaxed to the uniform stationary state.

A completely different scenario is observed when the two spirals are co-rotating. For intermediate values of the coupling strength $(2.9>\kappa>0.03)$, complex synchronization behavior is observed, as shown in Figs. 4.13 and 4.14. If the initial distance between the spiral tips is chosen sufficiently large $\left(d_{i}>d_{i}^{c}\right)$, the tips drift on opposite sides of an approximately circular trajectory, leading to a nearly circular trajectory for the difference vector of the two tip positions, shown in Fig. 4.13 (b). The modulations visible in the trajectory result from the underlying meandering motion of the tips, as shown in Fig. 4.13 (a). A perfect circle is obtained when the spiral tips exhibit steady rotation in the uncoupled system. Upon varying the initial distance $d_{i}$, we find a discrete set of such attractors. For example, for the parameters chosen in Fig. $4.13, d_{i}^{c} \approx 12$ and only the average diameters $d_{\infty}=32.5,72.5,112.5,155, \ldots$. of the approximately circular trajectory are exhibited for $t \rightarrow \infty$, depending on the initial distance $d_{i}$. Similar trajectories of a spiral tip have been observed in feedbackcontrolled dynamics of meandering spirals, and have been coined "resonance attractors" [73]. A theory explaining the existence and the stability of these attractors has been developed in $[86,87]$. A similar approach may be used to understand the nature of attractors such as that shown in Fig. 4.13 (b).

For $d_{i}<d_{i}^{c}$, a different type of synchronized tip motion occurs. The spiral tips now perform a meandering motion around a common central point. The trajectory of the difference vector of the two tip positions is still localized but clearly deviates from a circular shape, as shown in Fig. 4.14 (a). This behavior resembles the "entrainment attractors" observed in feedback-controlled dynamics of meandering spirals [73]. For 

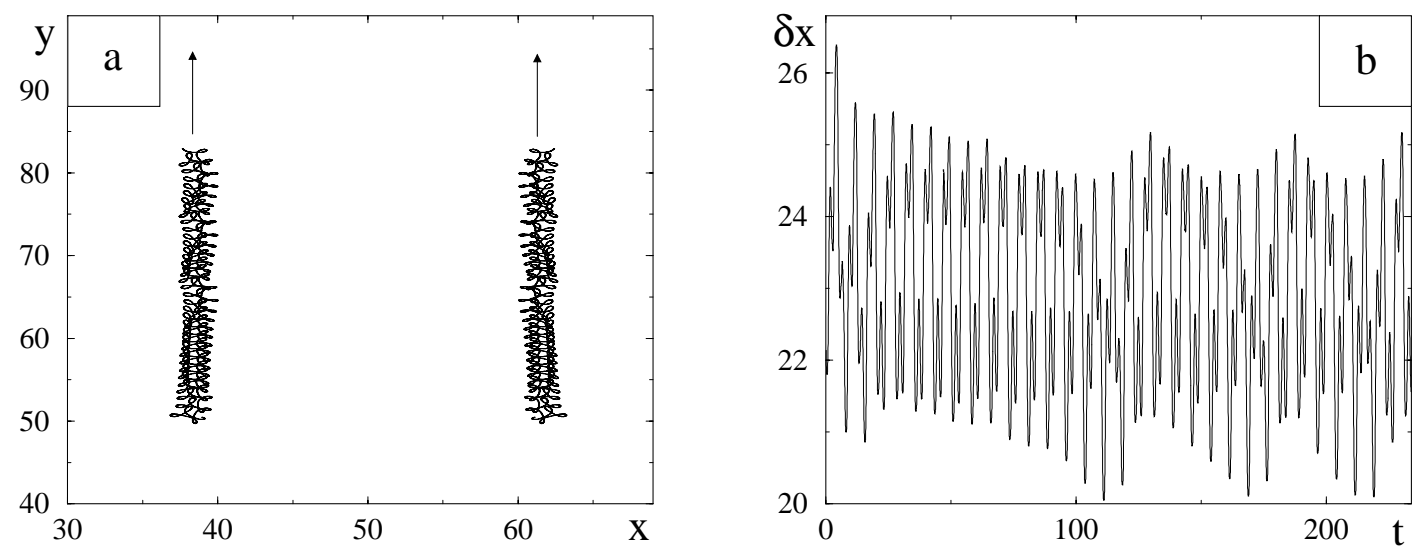

Figure 4.12. Results from numerical simulations of Eqs. (4.4) for a single spiral in each system. (a) Trajectories of spiral tips in the coupled systems 1 (left) and 2 (right), where the spirals rotate in opposite directions; $d_{i}=20, T=235.6$, and all other parameters as in Fig. 4.11. (b) Time series of the horizontal distance $\delta x$ between the spiral tips shown in (a). 

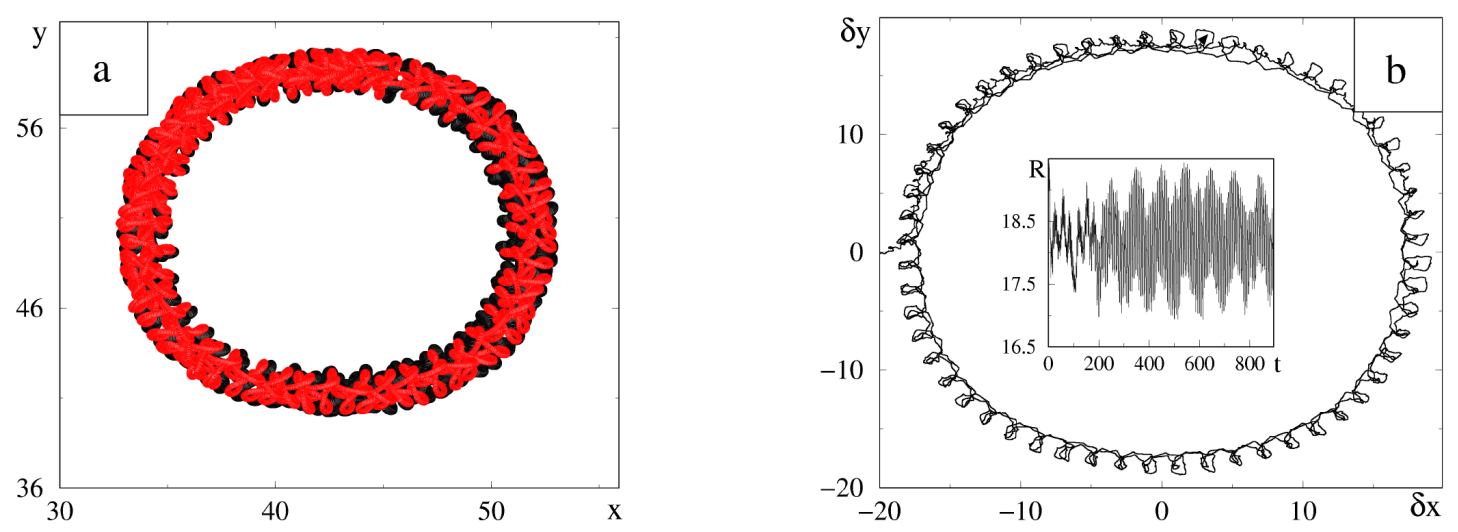

Figure 4.13. Results from numerical simulations of Eqs. (4.4) for a single spiral in each system. (a) Trajectories of spiral tips in the coupled systems 1 (black circles) and 2 (red circles), where the spirals rotate in same direction; $d_{i}=20, T=895.1$, and all other parameters as in Fig. 4.11. (b) Trajectories of the difference vector between the spiral tips in system 1 and system 2 for $d_{i}=20, T=895.1$. The insets show the corresponding time series of the distance $d(t)$ between the two tips. 

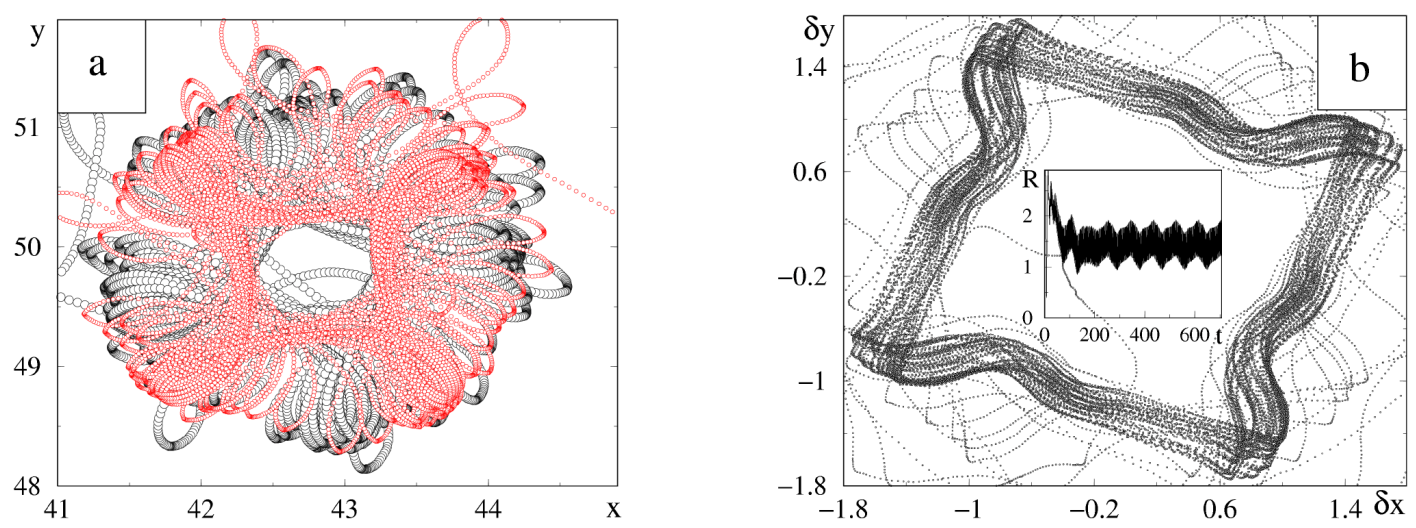

Figure 4.14. Results from numerical simulations of Eqs. (4.4) for a single spiral in each system. (a) Trajectories of spiral tips in the coupled systems 1 (black circles) and 2 (red circles), where the spirals rotate in same direction; $d_{i}=2.5, T=706.8$, and all other parameters as in Fig. 4.11. (b) Trajectories of the difference vector between the spiral tips in system 1 and system 2 . The insets show the corresponding time series of the distance $d(t)$ between the two tips. 
both large and small $d_{i}$, the spirals are destroyed by breakup for $\kappa>2.9$ and both systems relax to the uniform stationary state.

For other values of the system parameters, e.g., when the spirals perform steady rotation in the uncoupled system, a different kind of synchronization can be observed. Figures 4.15 (a) and (b) show tip trajectories with an irregular drift superimposed on the steady spiral tip rotation. A careful examination of the two trajectories shows that, at certain moments, the tip in system 1 stops its drift motion and exhibits a localized rotation for a brief period until the tip in system 2 closely approaches, whereupon the two spiral tips again begin to separate. The continuous repetition of this behavior leads to the formation of a complex flower pattern for the difference vector between the two tip positions, which is centered around $(0,0)$ [Fig. 4.15 (c)]. The weak synchronization of the two spirals is apparent in the time series of the tip distance shown in Fig. 4.15 (d). Quasi-mixed-mode oscillations are observed, with the period of the large-scale oscillation corresponding to the time needed for completing one of the petals shown in Fig. 4.15 (c) and the period of the small-scale oscillation being determined by the underlying rotational period of a spiral in the uncoupled system.

To gain further insights, we have systematically varied the coupling strength in numerical simulations of the coupled Barkley model, Eqs. (4.5), as shown in Fig. 4.16. We again observe a straight drift of counter-rotating spirals [Fig. 4.16 (a)]. In this case, however, the spirals do not annihilate at the boundary of the medium but become pinned and start to perform steady rotation. For sufficiently strong coupling, we find that the tips of co-rotating spirals lock on a nearly perfect circular attractor [Fig. 4.16 (b)]. As the coupling is decreased, the complex synchronization attractor appears [Fig. 4.16 (c),(d)]. The detailed behavior depends on the coupling strength $\kappa$. We have, however, performed a simulation for very weak coupling $\left(\kappa=10^{-4}\right)$, where we still observe the relaxation to an attractor with similar properties. 

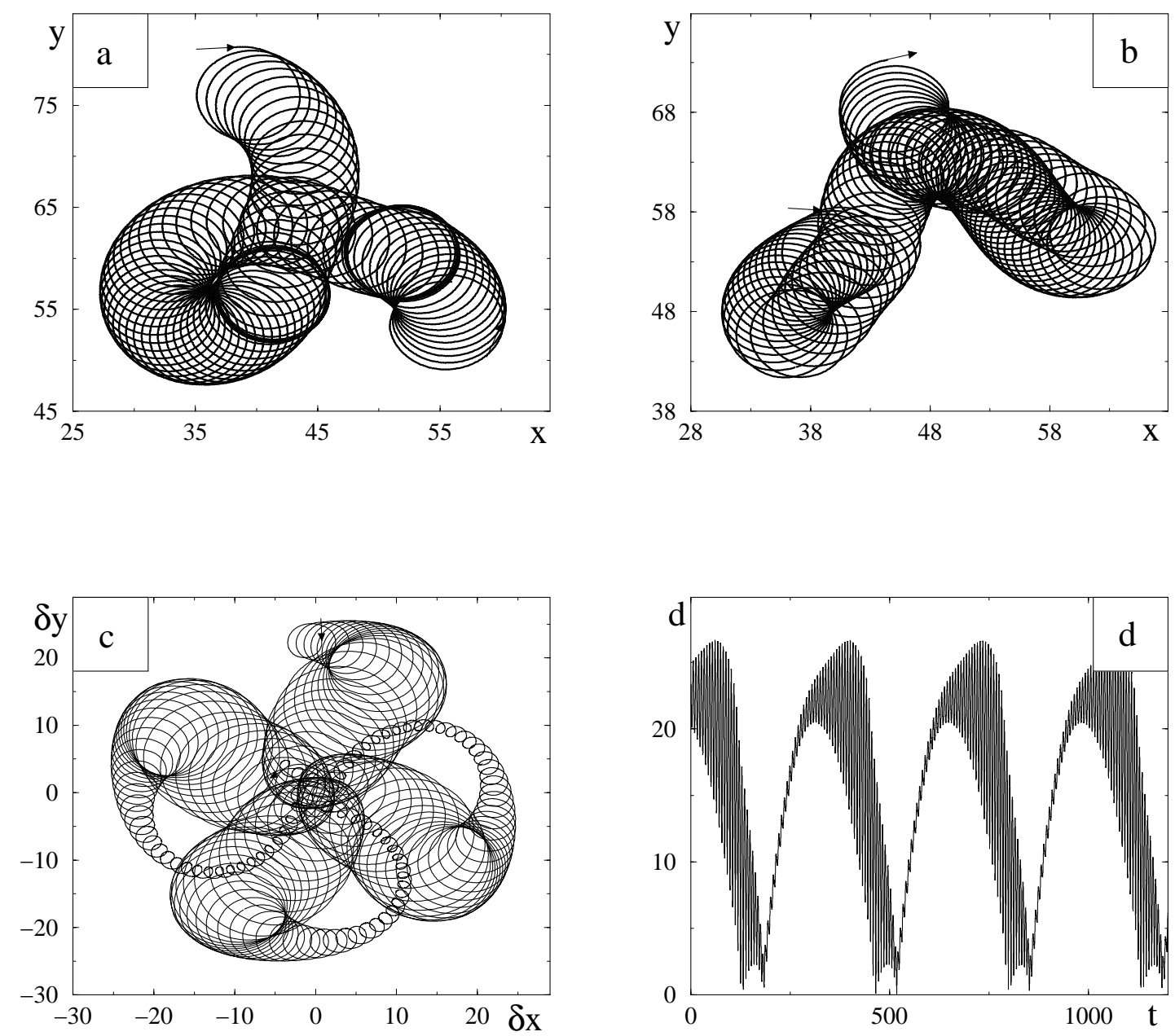

Figure 4.15. Results from a numerical simulation of Eqs. (4.4) with clockwise rotating spirals; $\phi_{0}=0.01, \kappa=1, \varepsilon=0.05, f=3.5, q=0.002, L=120$, and the initial spiral distance $d_{i}=7$. (a), (b) Trajectories of spiral tips in the coupled systems 1 (a) and 2 (b) for $T=602$. The arrows mark the beginning and end points of the trajectories. (c) Trajectory of the difference vector between the two tip positions. (d) Time series of the distance $d(t)$ between the two tips. $T=1203.8$ in (c) and (d). 

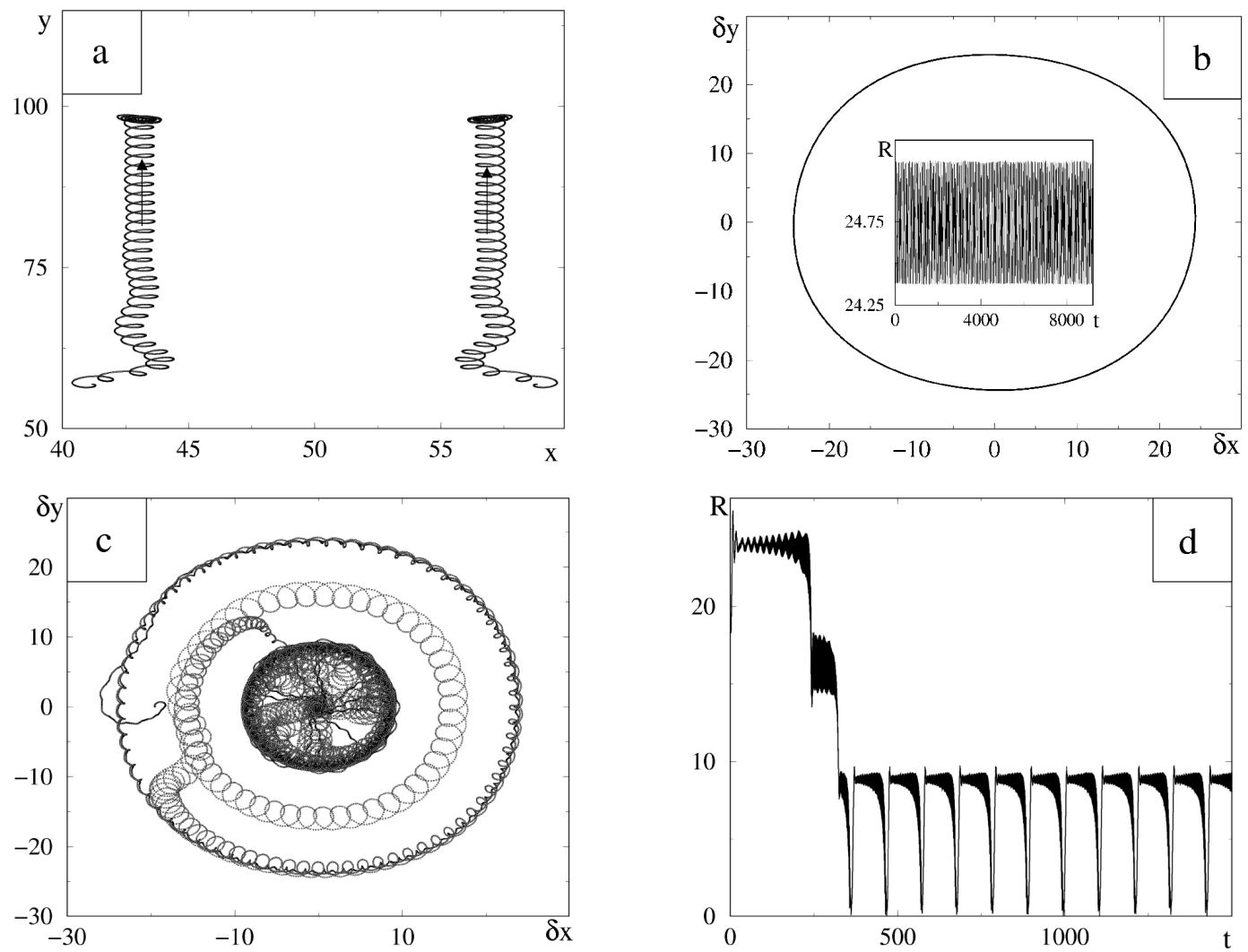

Figure 4.16. Results from a numerical simulation for the coupled Barkley system, Eqs. (4.5). (a) Trajectories of counter-rotating spiral tips in the coupled systems for $a=0.84$, $b=0.06, \epsilon=\kappa=0.03, d_{i}=19.6, L=100$, and $T=137$. (b) Trajectory of the difference vector between the two clockwise corotating spiral tips for $b=0.08, T=9227$, and all other parameters as in (a). The insets show the corresponding time series of the distance $d(t)$ between the two tips. (c) Trajectory of the difference vector between the two clockwise corotating spiral tips for $\kappa=0.02, T=7629$, and all other parameters as in (a). (d) Time series of the magnitude of the distance vector shown in (c). 


\subsection{Discussion and Conclusions}

We have studied two domains of excitable media that are locally coupled to each other by means of a video camera/projector through a coupling algorithm, which leads to weaker than identical synchronization. The coupling scheme is much more flexible than the diffusive cross-membrane coupling used in studies of identical synchronization of chemical wave patterns in a BZ system [79].

The relaxation of an initially disordered array of spirals to a synchronized state in two locally coupled excitable media in the photosensitive BZ reaction is qualitatively reproduced by numerical simulations of two locally coupled Oregonator systems. The complex synchronization behavior of fully developed single spirals has not allowed a direct comparison of experimental studies with the computational studies because of the difficulties in initially orienting the waves in the experiment as well as experimental fluctuations and parameter drift. Although the experimental behavior is in qualitative agreement with the numerical simulations, more refined experiments will be required for a quantitative comparison.

Note that in all of the cases shown in Figs. 4.12-4.15, the coupling induces a drift of the spiral tips, which is superimposed on the localized motion observed in the absence of coupling. Thus, for a given spiral in one of the irregular arrays shown in Figs. 4.6 and 4.11, the coupling leads to an increase in the translational motion of this spiral. Hence, the probability that its tip will meet another tip with opposite charge or that it will collide with the system boundary and thus be annihilated is increased. The total number of spirals therefore decreases much more rapidly than in the absence of coupling, where an initially disordered array of spirals is extremely long-lived as a result of exponentially weak interactions of the screened spiral tips [88].

The mutual synchronized state achieved from the initially disordered state is still complex in our study, which is a pervasive feature in biological systems. It has been pointed out that the mutual engagement of two or more elements with complex in- 
dividual dynamics into a fully synchronized state whose evolution is still complex is a relevant feature in the behavior of a wide class of natural systems [89], ranging from catalytic chemical reactions on surfaces [90] to biological systems such as neural networks [91] and insect populations [92]. It has also been emphasized that

mutual synchronization should play a significant role in the operation of the brain. The brain is essentially a system of interacting neural networks and the activity patterns of different networks may become synchronized while retaining their complex spatiotemporal dynamics [93]. Moreover, we note that the coupling in our study is multiplicative and symmetric, which might be anticipated in complex biological systems.

In summary, we have shown that the local coupling of two excitable media with complex spiral patterns gives rise to a reduction in spatial disorder, ultimately leading to a coherent pattern in each system corresponding to either a single spiral wave or to the uniform stationary state. Numerical simulations with a single spiral in each system show that instead of identical synchronization, different types of generalized synchronization attractors are exhibited. To our knowledge, this constitutes the first example of generalized synchronization in coupled spatially extended systems with non-chaotic dynamics.

\subsection{Appendix A: Synthesis of the Sulfato Salt of $\operatorname{Ru}(\mathrm{bpy})_{3}{ }^{2+}$}

The following is a representative procedure for the preparation of the sulfato salt of $\mathrm{Ru}(\mathrm{bpy})_{3}{ }^{2+}$. $11.4 \mathrm{~g}$ of bipyridine and $4.0 \mathrm{~g}$ of $\mathrm{Ru}(\mathrm{III}) \mathrm{Cl}_{3}$ were refluxed in 100 $\mathrm{ml}$ ethanol (95\%) for 72 hours. The mixture was filtered to remove the unreacted $\mathrm{RuCl}_{3}$. After drying, the unreacted $\mathrm{RuCl}_{3}$ was $0.6 \mathrm{~g}$. The funnel was washed with $50 \mathrm{ml}$ ethanol which was added to the filtered solution. The solution was distilled 
until it had approximately half of the original volume, $60 \mathrm{ml}$ of $6 \mathrm{M} \mathrm{H}_{2} \mathrm{SO}_{4}$ was then added and the mixture was placed in a freezer overnight. The crystals were filtered, washed with ether and dried in a vacuum desiccator. The powder is water soluble. Dissolving about $0.2 \mathrm{~g}$ of the powder in about $18 \mathrm{ml}$ water yields a solution of about $0.01 \mathrm{M}$. The exact concentration is then determined spectrophotometically, using the literature extinction coefficient of $\epsilon=13400 \mathrm{M}^{-1} \mathrm{~cm}^{-1}$ for $\mathrm{Ru}(\mathrm{bpy})_{3}{ }^{2+}$ at its maximum absorbance at $454 \mathrm{~nm}$ according to the Beer-Lambert law.

\subsection{Appendix B: Main Part of the Program in OP- TIMAS Used for the Experiments}

We define:

- bg as the homogeneous background, which is used for saving image;

- $\kappa$ as the coupling strength, which equal to coupling-setting/255;

- input1, input2 as the gray level of system1, system2 respectively;

- output1, output2 as the output light intensity for system1, system2 respectively;

Here, bg, coupling-setting, input1, input2, output1, and output2 are in the range of $0-255$. The calculation of output is based on the following equations:

output1 $=$ bg $+(\text { input1-input2) })^{*} \kappa$

output $2=\operatorname{bg}+($ input2-input 1$) * \kappa ;$

We set output1 (or output2) to 255 if it is greater than 255 and we set it to 0 if it is less than 0 .

If we think bg as $I_{0}$, input1/bg as $v_{1}$, input2/bg as $v_{2}$, we can rewrite Eqs.(B1) in a similar way as the equations $(1),(2)$ in [94]:

output1 $=$ bg $\left(1+(\text { input1-input2 })^{*} \kappa / b g\right) ;$ 
output2 $=$ bg $\left(1-(\text { input1-input2 })^{*} \kappa /\right.$ bg $) ;$

Here, we give calculation examples for different $\kappa$ :

If we set $\kappa$ as $0.31, \mathrm{bg}$ as 100 :

- case 1) Input1=255, Input2=0;

output $1=100+(255-0) * 0.31=179$;

output $2=100+(0-255) * 0.31=21$;

- case 2) Input1=50, Input2=170;

output $1=100+(50-170) * 0.31=63$;

output $2=100+(170-50) * 0.31=137$;

- case 3) Input1=100; Input2=100;

output1 $=100+(100-100) * 0.31=100 ;$

output $2=100+(100-100) * 0.31=100 ;$

If we set $\kappa$ as $1, \mathrm{bg}$ as 100

- case 1) Input1=255, Input2=0;

output $1=100+(255-0) * 1=355$;

output $1=255$; (since it is greater than 255.)

output $2=100+(0-255) * 1=-155$;

output $2=0 ;$ (since it is less than 0 .)

- case 2) Input1=50, Input2 $=170$; output1 $=100+(50-170) * 1=-20$;

output $1=0 ;($ since it is less than 0 .)

output $2=100+(170-50)^{*} 1=220$;

- case 3) Input1=100; Input2=100; output1 $=100+(100-100)^{*} 1=100$;

output $2=100+(100-100) * 1=100 ;$ 


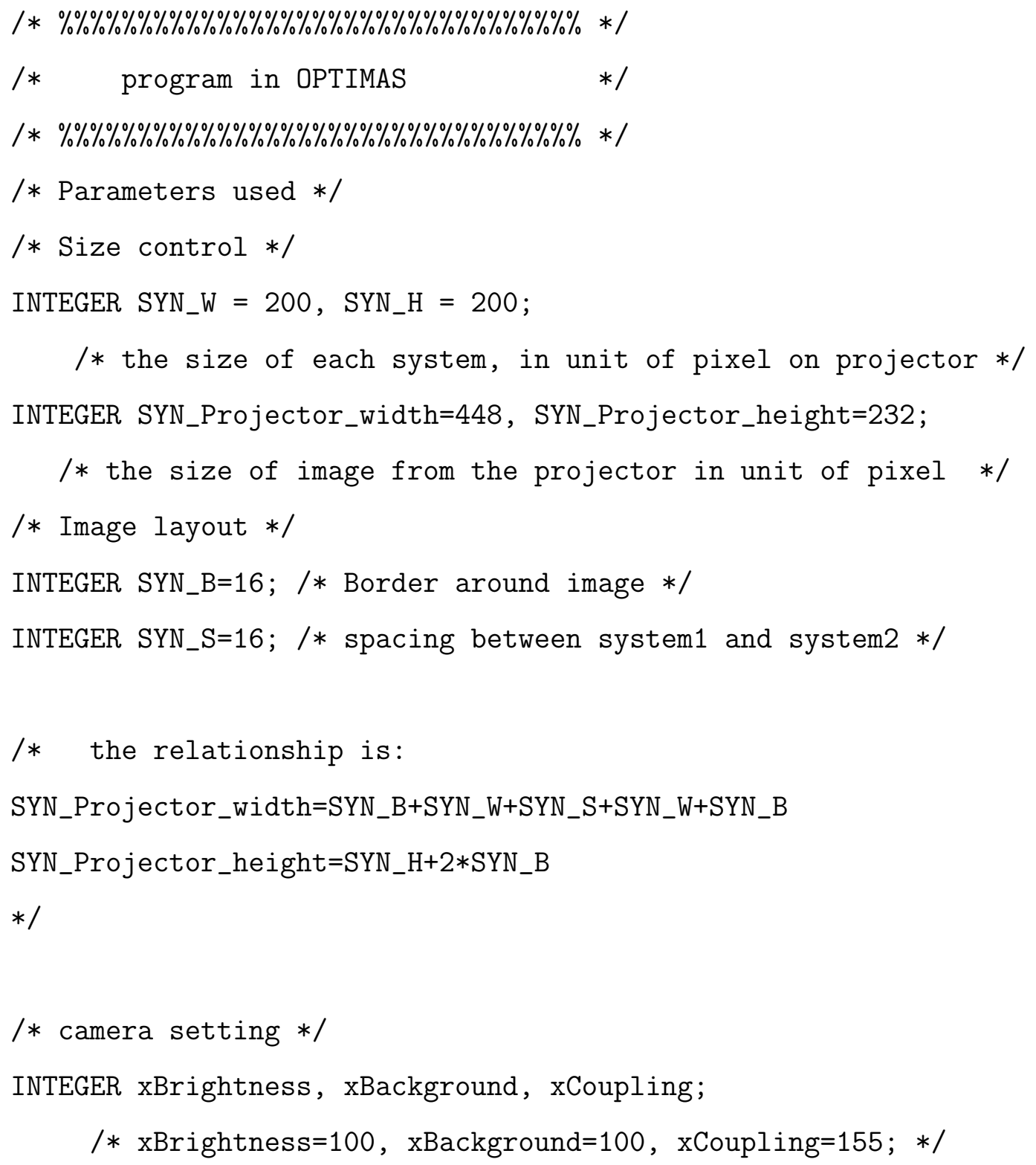


INTEGER xcontrast_range $=0: 255: 160$, Xcontrast; $/ /=155$;

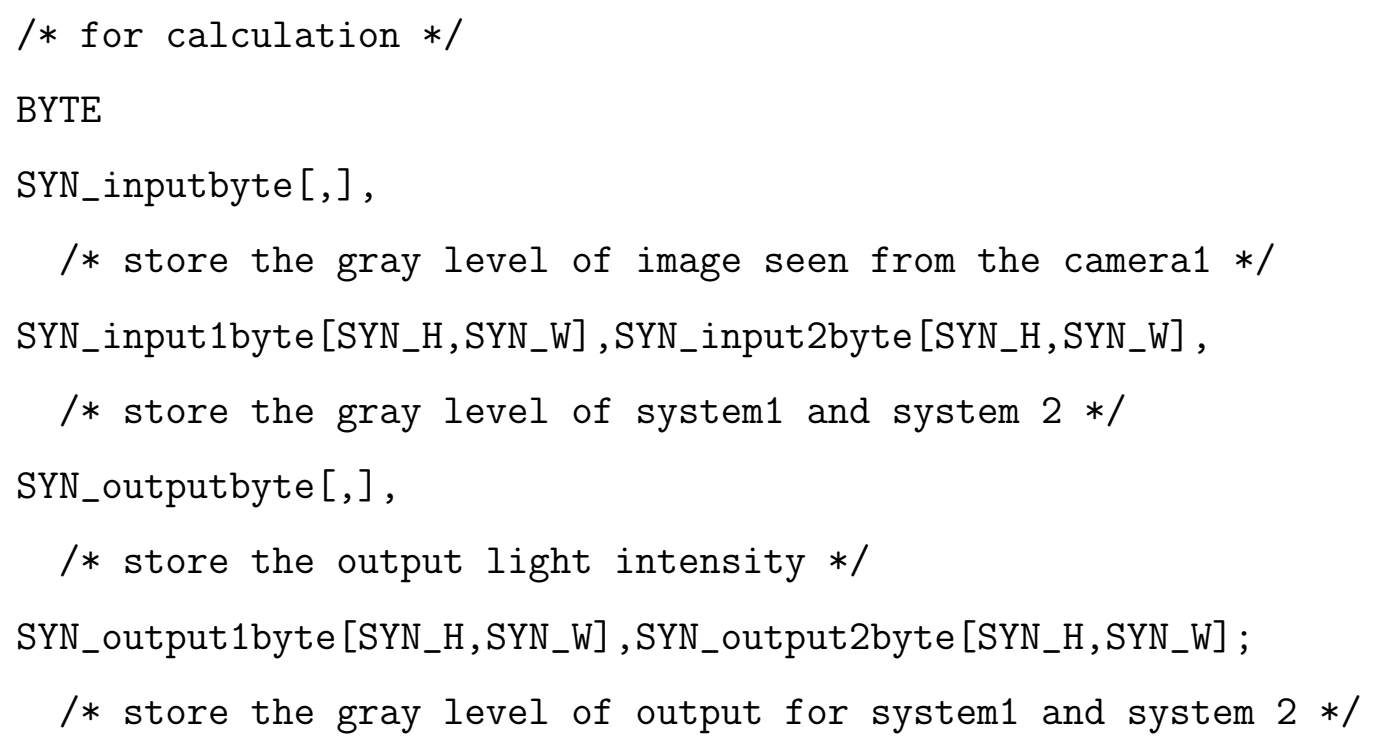

REAL

SYN_output1REAL [SYN_H ,SYN_W] , SYN_output2REAL [SYN_H , SYN_W] ;

/* store the gray level of output for system 1 and system $2 *$ / SYN_max1 $=255.0$, SYN_min1 $=0.0$;

/* control the range of output */ SYN_Coupling_Strength $=($ REAL $)$ xcoupling/255.0;

/* coupling strength of the experiment */

/* following arrays used in the whole process */

BYTE SYN_SaveBackground [SYN_projector_Height,SYN_projector_Width] ;

BYTE SYN_store_SaveBackground [SYN_projector_Height,SYN_projector_Width] ;

/* both store the homogeneous backgroud for projector */

BYTE SYN_PerturbationFinal[,];

/* store the output of pertubation for prpjector */ 
BYTE SYN_store_homo_array [,] ;

$$
\text { /* store the homo_array from camera2 */ }
$$

BYTE SYN_yWhite[SYN_Projector_width, SYN_Projector_height] $=180$;

$$
\text { /* the boundary setting */ }
$$

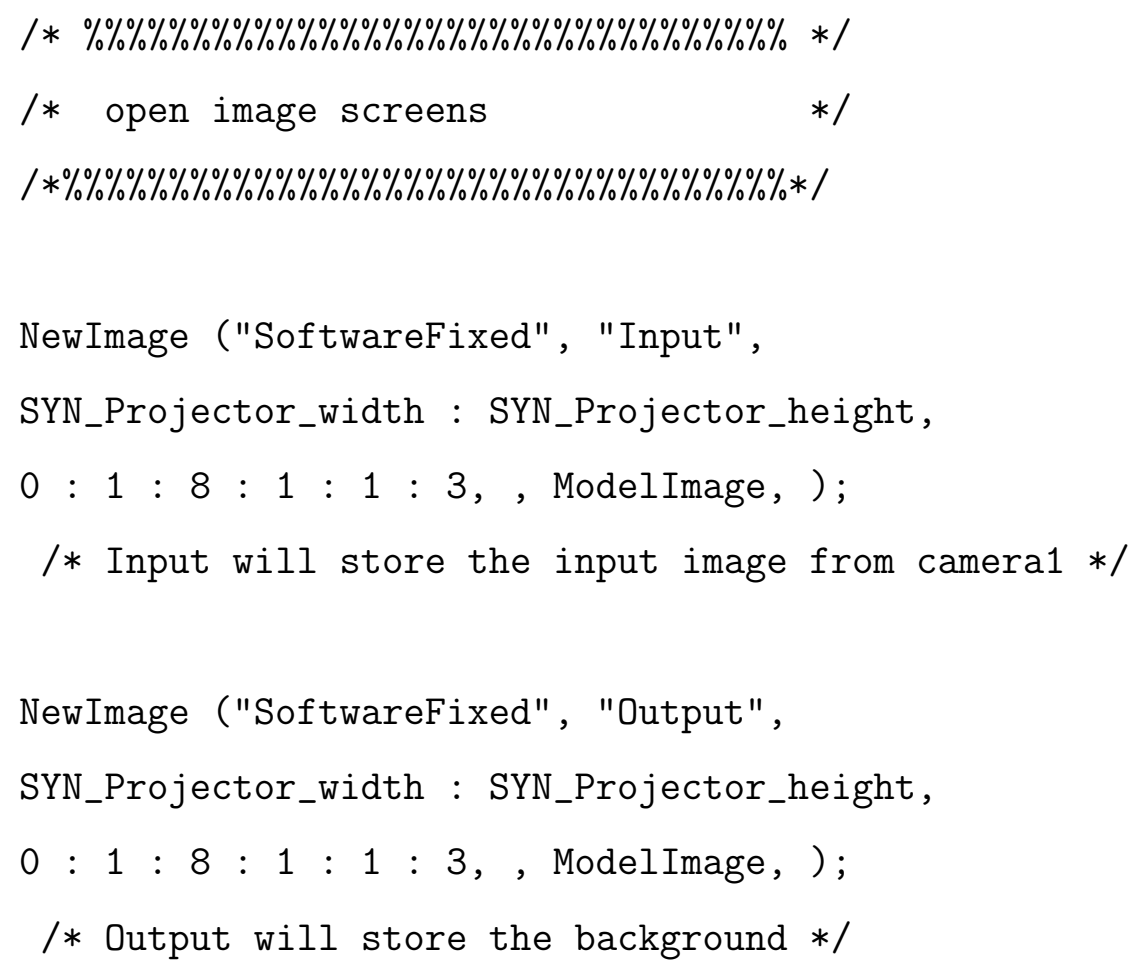




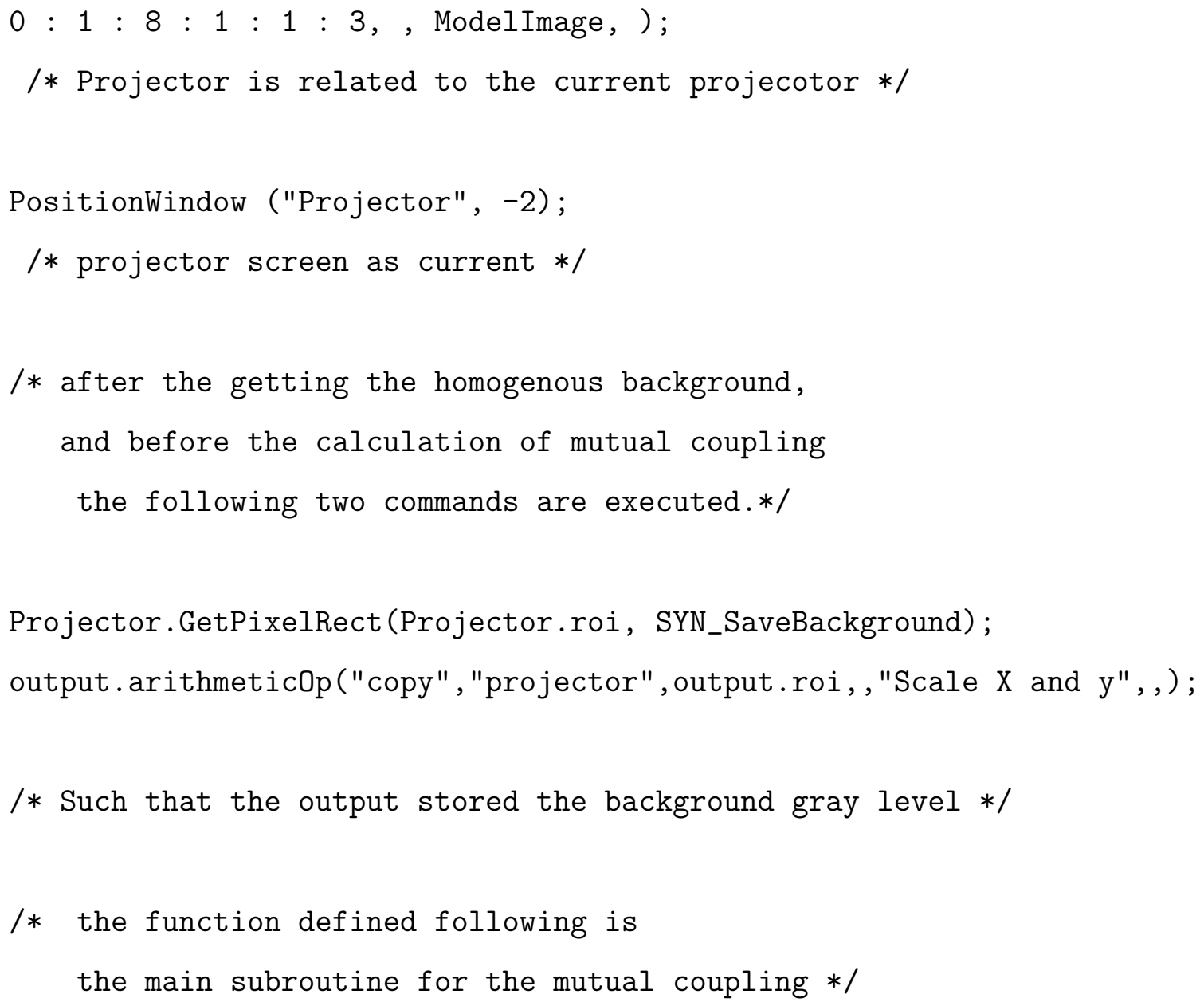




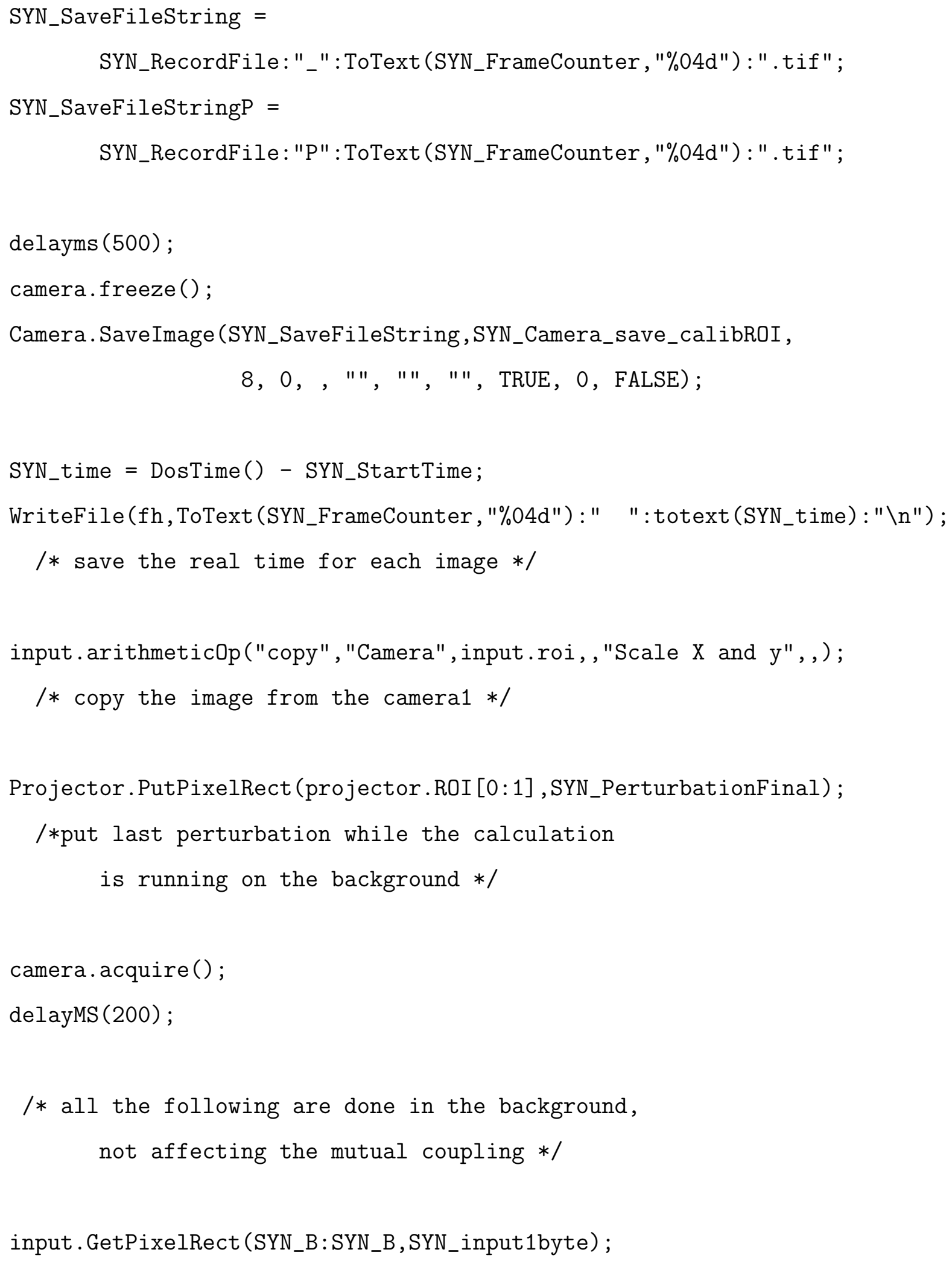




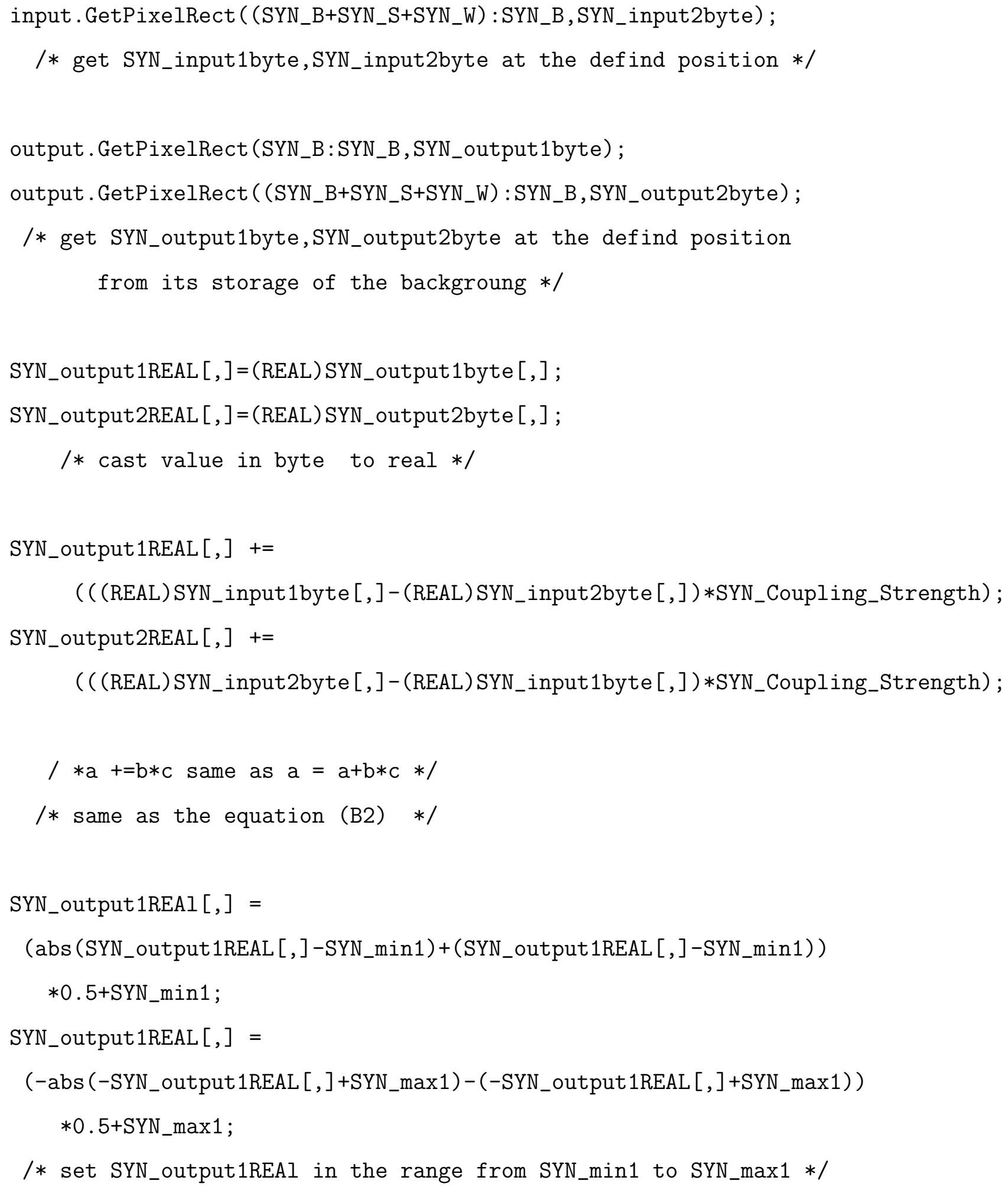




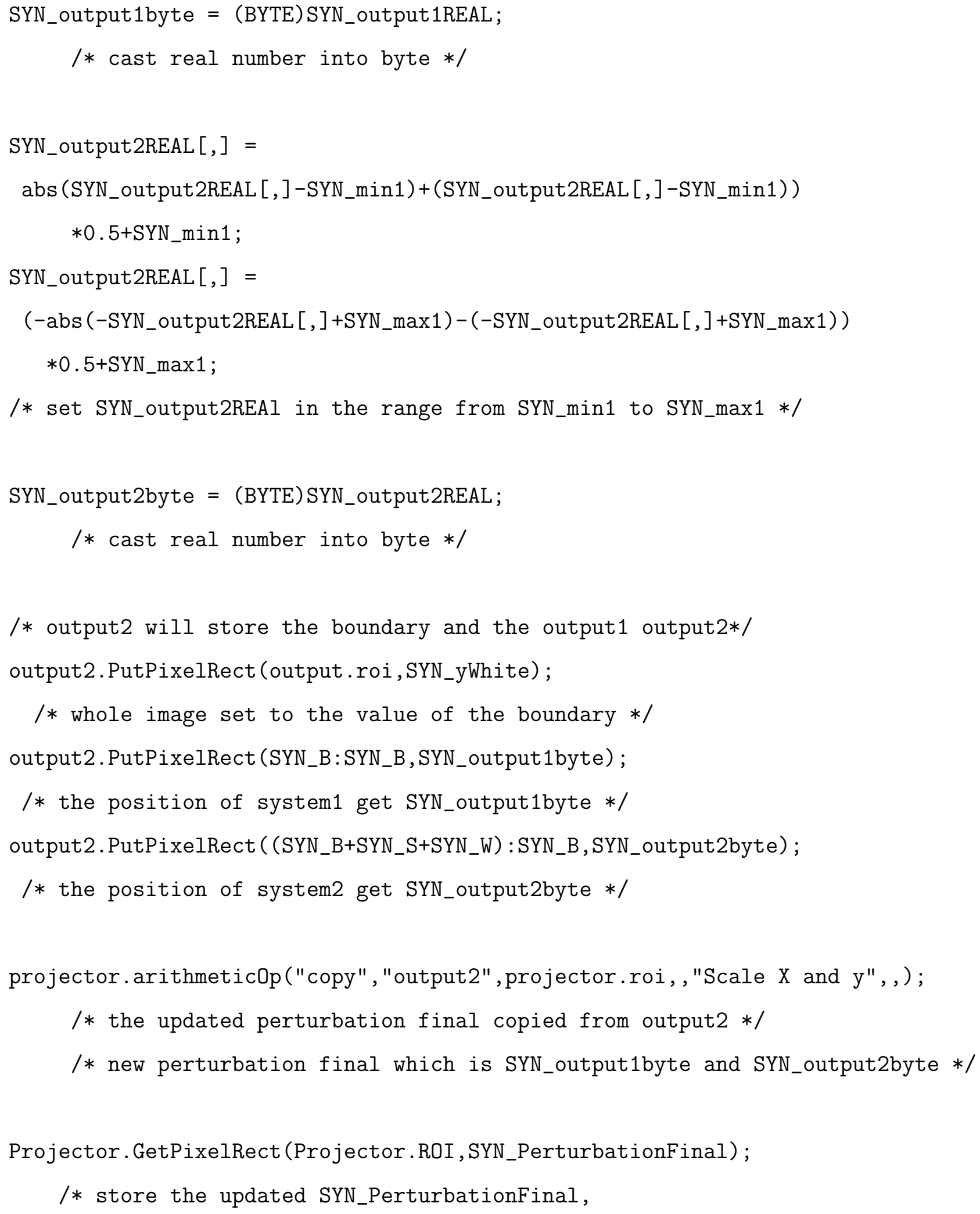


used for next loop while calculating */

Projector. SaveImage (SYN_SaveFileStringP, projector. ROI, 8, 0, , "", "", "", TRUE, 0, FALSE);

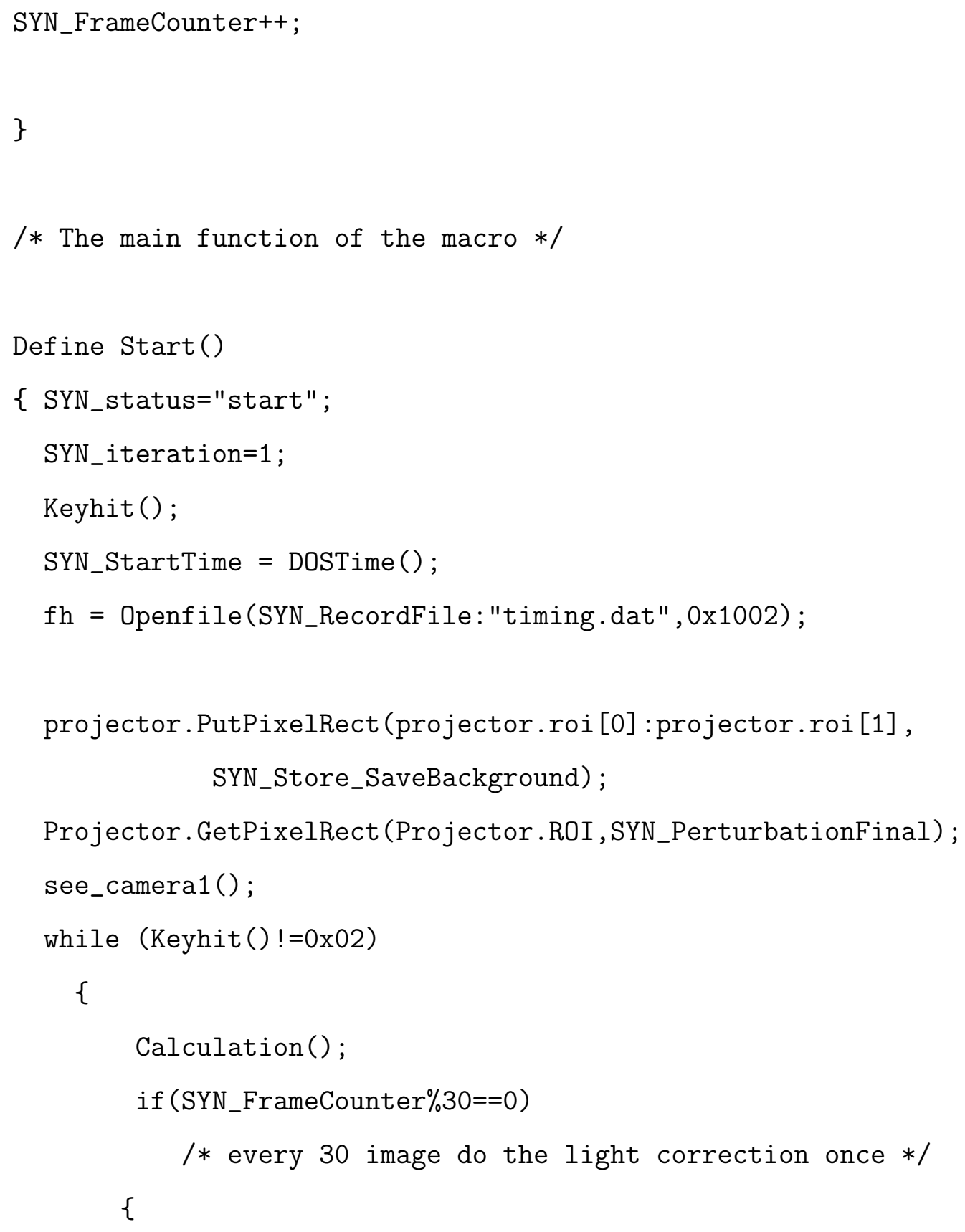




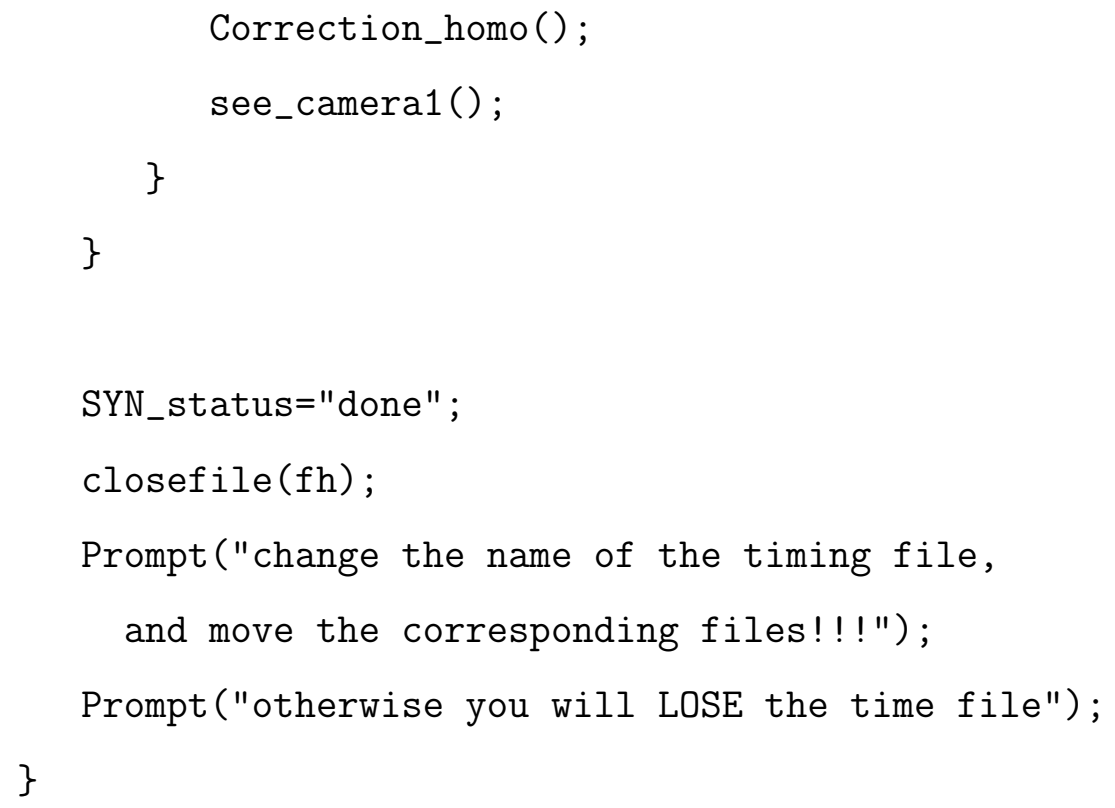




\subsection{References}

[1] A. S. Hornby, Oxford Advanced Dictionary (Oxford University Press, 1974).

[2] Ch. Huygens (Hugenii), Horologium Oscillatorium Apud F. Muguet, Parisiis, France, 1673. English translation: The Pendulum Clock (Iowa State University Press, Ames, 1986).

[3] S. H. Strogatz and I. Stewart, "Coupled Oscillators and Biological Synchronization," Sci. Am. 269, 102-109 (1993).

[4] A. V. Gaponov-Grekhov and M. I. Rabinovich, "Overview: Synchronization and Patterns in Complex Systems", Chaos 6, 259-261 (1996).

[5] H. Fujisaka and T. Yamada, "Stability Theory of Synchronized Motion in Coupled-Oscillator Systems," Prog. Theor. Phys. 69, 32-47 (1983).

[6] A. S. Pikovsky, "On the Interaction of Strange Attractors," Z. Phys. B 55, 149154 (1984).

[7] L. M. Pecora and T. L. Carroll, "Synchronization in Chaotic Systems," Phys. Rev. Lett. 64, 821-824 (1990).

[8] W. L. Ditto and K. Showalter, "Introduction: Control and Synchronization of Chaos," Chaos, 7, 509-511 (1997).

[9] Special issue, Phase Synchronization and its Applications, edited by J. Kurths, Int. J. Bifurcation Chaos Appl. Sci. Eng. 10-11 (2000);

[10] A. Pikovsky, M. Rosenblum, and J. Kurths, Synchronization-A Universal Concept in Nonlinear Sciences (Cambridge University Press, Cambridge, UK, 2001).

[11] S. Boccaletti, J. Kurths, G. Osipov, D. L. Valladares, and C. S. Zhou, "The Synchronization of Chaotic Systems," Phys. Reports 366, 1-101 (2002). 
[12] L. M. Pecora and T. L. Carroll, "Master Stability Functions for Synchronized Coupled Systems," Phys. Rev. Lett. 80, 2109-2112 (1990).

[13] M. G. Rosenblum, A. S. Pikovsky, and J. Kurths, "Phase Synchronization of Chaotic Oscillators", Phys. Rev. Lett. 76, 1804-1807 (1996).

[14] M. G. Rosenblum, A. S. Pikovsky, and J. Kurths, "From Phase to Lag Synchronization in Coupled Chaotic Oscillators," Phys. Rev. Lett. 78, 4193-4196 (1997).

[15] N. F. Rulkov, M. M. Sushchik, L. S. Tsimring, and H. D. I. Abarbanel, "Generalized Synchronizaton of Chaos in Directionally Coupled Chaotic Systems," Phys. Rev. E. 51, 980-994 (1995).

[16] M. A. Zaks, E.-H. Park, M. G. Rosenblum, and J. Kruths, "Alternating Locking Rations in Imperfect Phase Synchronization," Phys. Rev. Lett. 82, 4228-4231 (1999).

[17] R. Femat and G. Solis-Perales, "On the Chaos Synchronization Phenomena," Phys. Lett. A 262, 50-60 (1999).

[18] C. Zhou and J. Kurths, "Noise-Induced Phase Synchronization and Synchronization Transitions," Phys. Rev. Lett. 88, 230602(1-4) (2002).

[19] C. Schäfer, M. G. Rosenblum, J. Kurths, and H.-H. Abel, "Heartbeat Synchronized with Ventilation," Nature 392, 239-240 (1998).

[20] P. Tass, M. Rosenblum, J. Weule, J. Kurths, A. Pikovsky, J. Volkmann, A. Schnitzler, and H. Freund, "Detection of n:m Phase Locking from Noisy Data: Application to Magnetoencephalography," Phys. Rev. Lett. 81, 32913294 (1998). 
[21] A. Neiman, X. Pei, D. Russell, W. Wojtenek, L. Wilkens, F. Moss, H. A. Braun, M. T. Huber, and K. Voigt, "Synchronization of the Electrosensitive Cells in the Paddlefish", Phys. Rev. Lett. 82, 660-663 (1999).

[22] K. M. Cuomo and A. V. Oppenheim, "Circuit Implementation of Synchronized Chaos with Applications to Communications," Phys. Rev. Lett. 71, 65-68 (1993).

[23] G. D. VanWiggeren and R. Roy, "Communication with Chaotic Lasers," Science 279, 1198-1200 (1998).

[24] M. Peil, T. Heil, I. Fisher, and W. Elsäbetaer, "Synchronization of Chaotic Semiconductor Laser System: A Vectorial Coupling-Dependent Scenario," Phys. Rev. Lett. 88, 174101(1-4) (2002).

[25] V. Petrov, V. Gáspár, J. Masere, and K. Showalter, "Controlling Chaos in the Belousov-Zhabotinsky Reaction," Nature 361, 240-243 (1993).

[26] R. Roy, T. W. Murphy Jr., T. D. Maier, Z. Gills, and E. R. Hunt, "Dynamical Control of a Chaotic Laser: Experimental Stabilization of a Globally Coupled Systems," Phys. Rev. Lett. 68, 1259-1262 (1992).

[27] E. R. Hunt, "Stabilizing High-Period Orbits in a Chaotic System: The Diode Resonator," Phys. Rev. Lett. 67, 1953-1955 (1991).

[28] W. L. Ditto, S. N. Rauseo, and M. L. Spano, "Experimental Control of Chaos," Phys. Rev. Lett. 65, 3211-3214 (1990).

[29] M. C. Cross and P. C. Hohenberg, "Pattern Formation Outside of Equilibrium," Rev. Mod. Phys. 65, 851-1112 (1993).

[30] D. H. Zanette and A. S. Mikhailov, "Mutual Synchronization in Ensembles of Globally Coupled Neural Networks," Phys. Rev. E 58, 872-875 (1998). 
[31] L. Junge and U. Parlitz, "Synchronization and Control of Coupled GinzburgLandau Equations Using Local Coupling," Phys. Rev. E 61, 3736-3742 (2000).

[32] S. J. Schiff, P. So, T. Chang, R. E. Burke, and T. Sauer, "Detecting Dynamical Interdependence and Generalized Synchrony Through Mutual Prediction in a Neural Ensemble," Phys. Rev. E 54, 6708-6724 (1996).

[33] L. Kocarev, Z. Tasev, T. Stojanovski, and U. Parlitz, "Synchronizing Spatiotemporal Chaos," Chaos 7, 635-643 (1997).

[34] L. Kocarev and U. Parlitz, "Synchronizing Spatiotemporal Chaos in Coupled Nonlinear Oscillators," Phys. Rev. Lett. 77, 2206-2209 (1996).

[35] S. Boccaletti, D. L. Valladares, J. Kurths, D. Maza, and H. Mancini, "Synchronization of Chaotic Structurally Nonequivalent Systems," Phys. Rew. E 61, 3712-3715 (2000).

[36] A. Amengual, E. Hernándze-García, R. Montagne, and M. S. Miguel, "Synchronization of Spatiotemporal Chaos: The Regime of Coupled Spatiotemporal Intemittency," Phys. Rev. Lett. 78, 4379-4382, (1997).

[37] V. S. Anishchenko, A. B. Neiman, F. Moss, and L. Schimansky-Geier, "Stochastic Resonance: Noise-Enhaced Order," Phys. Uspekhi 42, 7-35 (1999).

[38] B. Shulgin, A. Neiman, and V. Anishchenko, "Mean Switching Frequency Locking in Stochastic Bistable Systems Driven by a Periodic Force," Phys. Rev. Lett. 75, 4157-4160 (1995).

[39] O. Steinkbock, V. S. Zykov, and S. C. Müller, "Control of Spiral-Wave Dynamics in Active Media by Periodic Modulation of Excitability," Nature 366, 322-324 (1993).

[40] H. Chaté, A. Pikovsky, and O. Rudzick, "Forcing Oscillatory Media: Phase Kinks vs. Synchronization," Physica D 131, 17-30 (1999). 
[41] A. Neiman, L. Schimansky-Geier, F. Moss, B. Shulgin, and J. J. Collins, "Synchronization of Noisy Systems by Stochastic Signal," Phys. Rev. E 60, 284-292 (1999).

[42] L. Gammaitoni, P. Hänggi, P. Jung, and F. Marchesoni, "Stochastic Resonance," Rev. Mod. Phys. 70, 223-287 (1998).

[43] C. Zhou, J. Kurths, I. Z. Kiss, and J. L. Hudson, "Noise-Enhanced Phase Synchronization of Chaotic Oscillators," Phys. Rev. Lett. 89, 014101(1-4) (2002).

[44] A. Neiman, L. Schimansky-Geier, A. Cornell-Bell, and F. Moss, "Noise-Enhaced Phase Synchronization in Excitable Media," Phys. Rev. Lett. 83, 4896-4899 (1999).

[45] A. B. Neiman and D. F. Russell, "Synchronization of Noise-Induced Bursts in Noncoupled Sensory Neurons." Phys. Rev. Lett. 88, 138103(1-4) (2002).

[46] L. G. Morelli and D. H. Zanette, "Synchronization of Stochastically Coupled Cellular Automata," Phys. Rev. E 58, R8-R11 (1998).

[47] P. Grassberger, "Synchronization of Coupled Systems with Spatiotemporal Chaos," Phys. Rev. E 59, R2520-R2522 (1999).

[48] V. Ahlers and A. Pikovsky, "Critical Properties of the Synchronization Transition in Space-Time Chaos," Phys. Rev. Lett. 88, 254101(1-4) (2002).

[49] S. Boccaletti, J. Bragard, and F. T. Arecchi, "Controlling and Synchronization Space Time Chaos," Phys. Rev. E 59, 6574-6578 (1999).

[50] L. Kocarev, Z. Tasev, and U. Parlitz, "Synchronizing Spatiotemporal Chaos of Partial Differential Equations," Phys. Rev. Lett. 79, 51-54 (1997).

[51] Cellular Automata: Theory and Experiment. edited by H. Gutowitz, (NorthHolland, Amsterdam, 1990). 
[52] G. Hu, J. Xiao, J. Yang, F. Xie, and Zh. Qu, "Synchronization of Spatiotemporal Chaos and Its Applications," Phys. Rev. E 56, 2738-2746 (1997).

[53] Y. Jiang and P. Parmananda, "Synchronization of Spatiotemporal Chaos in Asymmetrically Coupled Map Lattices," Phys. Rev. E 57, 4135-4139 (1998).

[54] A. L. Gelover-Santiago, R. Lima, and G. Martinez-Mekler, "Synchronization in Globally Coupled Maps", Int. J. Bifurcation Chaos Appl. Sci. Eng 10, 453-460 (2000).

[55] S. N. Chow, J. Mallet-Paret, and E. S. Van Vleck, "Dynamics of Lattice Differential Equations," Int. J. Bifurcation Chaos Appl. Sci. Eng 6, 1605-1621 (1996).

[56] S. Boccaletti, J. Bragard, F. T. Arecchi, and H. Mancini, "Synchronization in Nonidentical Extended Systems," Phys. Rev. Lett. 83, 536-539 (1999).

[57] L. Junge and U. Parlitz, "Phase Synchronization of Coupled Ginzburg-Landau Equations," Phys. Rev. E 62, 438-441 (2000).

[58] J. Bragard, S. Boccaletti, and F. T. Arecchi, "Control and Synchronizatioin of Space Extended Dynamical Systems," Int. J. Bifurcation Chaos Appl. Sci. Eng 11, 2715-2729 (2001).

[59] P. Parmananda, "Generalized Synchronization of Spatiotemporal Chemical Chaos," Phys. Rev. E 56, 1595-1598 (1997).

[60] Y. Kuramoto, Chemical Oscillations, Waves and Turbulence (Springer, Berlin, 1984).

[61] P. Gray and S. K. Scott, "Autocatalytic Reactions in the Isothermal, Continuous Stirred Tank Reactor; Oscillations and Instabilities in the System A + 2B $\rightarrow$ BB; B $\rightarrow$ C," Chem. Eng. Sci. 39, 1087-1097 (1984).

[62] Y. Kuramoto, "Instability and Turbulence of Wavefronts in Reaction-Diffusion Systems," Prog. Theor. Phys. 63, 1885-1903 (1980); 
[63] G. I. Sivashinsky, "Nonlinear Analysis of Hydrodynamic Instability in Laminar Flames-I. Derivation of Basic Equations," Acta Astronaut. 4, 1177-1206 (1977).

[64] M. K. Ram Reddy, Zs. Nagy-Ungvarai, and S. C. Müller, "Effect of Visible Light on Wave Propagation in the Ruthenium-Catalyzed Belousov-Zhabotinsky Reaction,” J. Phys. Chem. 98, 12255-12259 (1994).

[65] Z. Noszticzius, W. Horsthemke, W. D. McCormick, H. L. Swinney, and W. Y. Tam, "Sustained Chemical Waves in an Annular Gel Reactor: A Chemical Pinwheel," Nature 329, 619-620 (1987).

[66] B. Neumann, Zs. Nagy-Ungvarai and S. C. Müller, "Interaction Between Silica Gel Matrices and the Belousov-Zhabotinsky Reaction," Chem. Phys. Lett. 211, 36-40 (1993).

[67] T. Yamaguchi, L. Kuhnert, Zs. Nagy-Ungavarai, S. C. Müller, and B. Hess, "Gel Systems for the Belousov-Zhabotinskii Reaction," J. Phys. Chem. 95, 5831-5837 (1991).

[68] L. Kuhert, K. I. Agladze, and V. I. Krinsky, "Image Processing Using LightSensitive Chemical Waves," Nature 337, 244-247 (1989).

[69] O. Steinbock, V. Zykov and S. C. Müller, "Control of Spiral-Wave Dynamics in Active Media by Periodic Modulation of Excitability," Nature 366, 322-324 (1993).

[70] V. Petrov, Q. Ouyang, and H. L. Swinney, "Resonant Pattern Formation in a Chemical System," Nature 388, 655-657 (1997).

[71] A. L. Lin, A. Hagberg, A. Ardelea, M. Bertram, H. L. Swinney, and E. Meron, "Four-Phase Patterns in Forced Oscillatory Systems," Phys. Rev. E 62, 3790$3798(2000)$. 
[72] S. Kádár, J. Wang, and K. Showalter, "Noise-Supported Travelling Waves in Sub-Excitable Media," Nature 391, 770-772 (1998).

[73] S. Grill, V. S. Zykov, and S. C. Müller, "Feedback-Controlled Dynamics of Meandering Spiral Waves," Phys. Rev. Lett. 75, 3368-3371 (1995).

[74] M. Hildebrand, H. Skødt, and K. Showalter, "Spatial Symmetry Breaking in the Belousov-Zhabotinsky Reaction with Light-Induced Remote Communication," Phys. Rev. Lett. 87, 088303(1-4) (2001).

[75] V. K. Vanag, L. Yang, M. Dolnik, A. M. Zhabotinsky, and I. R. Epstein, "Oscillatory Cluster Patterns in a Homogeneous Chemical System with Global Feedback," Nature 406, 389-391 (2000).

[76] M. Dolnik and I. R. Epstein, "Coupled Chaotic Chemical Oscillators," Phys. Rev. E 54, 3361-3368 (1996).

[77] M. F. Crowley and I. R. Epstein, "Experimental and Theoretical Studies of a Coupled Chemical Oscillator: Phase Death, Multistability and In-Phase and Out-Of-Phase Entrainment," J. Phys. Chem. 93, 2496-2502 (1989).

[78] M. F. Crowley and R. J. Field, "Electrically Coupled Belousov-Zhabotinskii Oscillators. 1. Experiments and Simulations," J. Phys. Chem. 90, 1907-1915 (1986).

[79] D. Winston, M. Arora, J. Maselko, V. Gáspár, and K. Showalter, "CrossMembrane Coupling of Chemical Spatiotemporal Patterns," Nature 351, 132-135 (1991).

[80] S. Kádár, T. Amemiya, and K. Showalter, "Reaction Mechanism for Light Sensitivity of the $\mathrm{Ru}(\mathrm{bpy})_{3}{ }^{2+}$-Catalyzed Belousov-Zhabotinsky Reaction," J. Phys. Chem. A 101, 8200-8206 (1997).

[81] J. Wang, S. Kádár, P. Jung, and K. Showalter, "Noise Driven Avalanche Behavior in Subexcitable Media," Phys. Rev. Lett. 82, 855-858 (1999). 
[82] R. J. Field and R. M. Noyes, "Oscillations in Chemical Systems. IV. Limit Cycle Behavior in a Model of a Real Chemical Reaction," J. Chem. Phys. 60, 1877-1884 (1974).

[83] J. J. Tyson and P. C. Fife, "Target Patterns in a Realistic Model of the BelousovZhabotinskii Reaction," J. Chem. Phys. 73, 2224-2237 (1980).

[84] H. J. Krug, L. Pohlmann, and L. Kuhnert, "Analysis of the Modified Complete Oregonator Accounting for Oxygen Sensitivity and Photosensitivity of BelousovZhabotinsky Systems," J. Phys. Chem. 94, 4862-4866 (1990).

[85] D. Barkley, "A Model for Fast Computer Simulation of Waves in Excitable Media," Physica D 49, 61-70 (1991).

[86] A. Karma and V. S. Zykov, "Structure of the Resonance Attractor for Spiral Waves in Excitable Media," Phys. Rev. Lett. 83, 2453-2456 (1999).

[87] V. S. Zykov, O. Kheowan, O. Rangsiman, and S. C. Müller, "Instabilities of the Resonance Attractor for Spiral Waves in an Excitable Medium," Phys. Rev. E 65, 026206(1-8) (2002).

[88] I. S. Aranson, L. Kramer, and A. Weber, "On the Interaction of Spiral Waves in Non-Equilibrium Media," Physica D 53, 376-384 (1991).

[89] Y. Kuramoto, Chemical Oscillations, Waves and Turbulence (Springer, Berlin, 1984).

[90] N. Khrustova, G. Veser, A. Mikhailov, and R. Imbihl, "Delay-Induced Chaos in Catalytic Surface Reactions: NO Reduction on Pt(100)," Phys. Rev. Lett. 75, 3564-3567 (1995).

[91] D. Golomb, D. Hansel, B. Shraiman, and H. Sompolinsky, "Clustering in Globally Coupled Phase Oscillators," Phys. Rev. A 45, 3516-3530 (1992) 
[92] K. Kaneko, "Globally Coupled Circle Maps," Physica D 54, 5-19 (1991).

[93] H. D. Abarbanel, M. I. Rabinovich, A. Seleverston, M. V. Bazhenov, R. Huerta, M. M. Sushchik, and L. L. Rubchinskii, "Synchronisation in Neural Networks," Phys. Usp. 39, 337-362 (1996).

[94] M. Hildebrand, J. Cui, E. Mihaliuk, J. Wang, and K. Showalter, "Synchronization of Spatiotemporal Patterns in Locally Coupled Excitable Media," Phys. Rev. E , 68, 026205-1-4 (2003). 


\section{Chapter 5}

\section{Modeling Disease Spreading with Excitable Media}

In this chapter, we review modeling studies on disease spreading and describe how we use excitable media for such studies. We present both experimental and simulation results.

\subsection{Reviews of Modeling Disease Spreading}

\subsubsection{A brief history of modeling disease spreading}

Epidemics have been occurring since human beings began to form communities. Often, epidemics reoccur at regular intervals of several years between outbreaks. Descriptions of epidemics in ancient and mediaeval times frequently used the term plague because of a general belief that they were divine retribution for sinful living [1]. Studies of diseases spreading can be dated back to 1662, when John Graunt carried out a quantitative study of human diseases and deaths ensuing from them [2]. The first known result in mathematical epidemiology is a defense of the practice of inocula- 
tion against smallpox in 1760 by Daniel Bernouilli [3], a member of a famous family of mathematicians (eight spread over three generations) who had been trained as a physician [1]. William Farr attempted to mathematically characterize the smallpox death data in 1840 [4] and predict the spread of rinderpest among cattle in 1866 (quoted in [5]). By the beginning of the twentieth century, the idea of passing on a bacterial disease through contact between susceptible and infective had become familiar. W. H. Hamer adapted the simple 'mass action' principle, a principle for chemical reaction, which is stated as "for a homogeneous system, the rate of a chemical reaction is proportional to the active masses of the reacting substances," for a deterministic epidemic model in discrete time in 1906 [6]. In 1916, R. Ross [7] used continuous time versions of the epidemic model in his studies of populations subject to infection. However, the most commonly used models to characterize the typical general epidemic is due to W. O. Kermack and A. G. McKendrick [8]. The modeling literature is now dominated by the influence of the theoretical paper on epidemic models by Kermack and Mckendrik. One can find detailed descriptions of the development of mathematical theories for the spread of disease $[5,9,10]$.

\subsubsection{Mathematical epidemiology models}

In 1927, W. O. Kermack and A. G. McKendrick [8] proposed the formulation of a model for disease spreading, which represented a fundamental milestone of mathematical epidemiology. In order to model an epidemic whose victims recovered with immunity against reinfection, the populations are divided into three classes, labeled $S, I$, and $R$. $S$ denotes the number of individuals who are susceptible to the disease, that is, who are not yet infected. I denotes the number of infected individuals, assumed infectious and able to spread the disease by contact with susceptible. $R$ denotes the number of individuals who have been infected and then removed from the possibility of being infected again or of spreading infection through isolation from the rest of the population or through immunization against infection or through recovery 
from the disease with full immunity against reinfection or through death caused by the disease. These characterizations of removed members are very different from an epidemiological perspective but are equivalent from a modeling point of view which takes into account only the state of an individual with respect to the disease. It is described as the SIR model because the transitions are from susceptible to infective to removed, with the removal coming through recovery with full immunity (as in measles) or through death from the disease (as in plague, rabies and many other animal diseases). The model predicted behavior very similar to the behavior observed in countless epidemics. Here, we present the SIR model in a homogeneous system as an example. The SIR model can be described in terms of population size of susceptible, infected, and removed individuals, $S, I$, and $R$ respectively, where a fixed population size $N$ is assumed. Often times, $S(t), I(t)$, and $R(t)$ describe the densities of susceptible, infected, and removed individuals as a function of time. These magnitudes are linked through the normalization condition

$$
S(t)+I(t)+R(t)=1,
$$

and using the homogeneous mixing principle for continuous time $(t \geq 0)$, they obey the following system of differential equations:

$$
\begin{aligned}
\frac{d S}{d t} & =-\lambda \bar{k} I S, \\
\frac{d I}{d t} & =-\mu I+\lambda \bar{k} I S, \\
\frac{d R}{d t} & =\mu I .
\end{aligned}
$$

Here, $\lambda$ is the infection spreading rate, $\bar{k}$ is the number of contacts per unit time that is supposed to be constant for the whole population, and $\mu$ is the rate coefficient for infected individuals to decay into the removed class.

The most significant prediction of this model is the presence of a nonzero epidemic threshold $\lambda_{c}$. If the value of $\lambda$ is larger than $\lambda_{c}$, the disease spreads and infects a 
finite fraction of the population. On the other hand, when $\lambda$ is below the threshold $\lambda_{c}$, the total number of infected individuals is infinitesimally small in the limit of very large populations. The typical size of outbreaks of the epidemic and whether or not epidemics occur are the main topics for the classic mathematic modeling with the assumption of large population size.

This threshold theorem was established for the first time by Kermack and McKendrick in 1927 [8]. In some other contexts, threshold phenomena in epidemiology are rephrased as the transmission probability to exceed a threshold when the spreading rate of infection is thought of in terms of a transmission probability [9]. The threshold theorem is important because it implies what proportion of susceptibles need to be vaccinated in order to prevent an epidemic from occurring. Epidemic behavior usually shows a phase transition from a regime without epidemics to one with epidemics. This transition happens as the "reproductive ratio" of the disease, which is the fractional increase per unit time in the number of infective individuals, becomes greater than one [9].

The SIR model is a deterministic model that describes the progress of a disease determined entirely by the current state and possibly by the past history, but with no allowance for random effects. When population sizes are large, this is a reasonable approximation, but for small population sizes individual differences and random effects are important. Stochastic models are needed to describe the disease spreading in small populations, in particular those of family size [1]. The evolution of an epidemic described as a stochastic process can be found in Refs. $[5,11]$.

Another model proposed by W. O. Kermack and A. G. McKendrick is an SIS model [12], in which infectious return to the susceptible class on recovery because the disease confers no immunity against reinfection. Such models are appropriate for most diseases transmitted by bacterial or helminth agents, and most sexually transmitted diseases (including gonorrhea, but not such diseases as AIDS from which there is no recovery). 
In many diseases, individuals do not pass directly from the susceptible to the infective class on becoming infected. There may be a latent or exposed stage. If the exposed period is short, as influenza has an exposed period of 1-3 days, it is often neglected in modeling, but a longer exposed period could lead to significantly different model predictions $[1,13]$. The inclusion of an exposed period would mean an additional compartment $E$ in the model, thus SEIR or SEIS models are used $[1,13]$. Fox rabies, for example, has an exposed period (the incubation period) of 28-30 days considerably longer than the actual infective period $[1,13]$. In measles there is an incubation period of 11-14 days after infection before symptoms of the disease appear $[1,13]$.

Some diseases, such as malaria, provide a temporary immunity on recovery, so that there is a transition from the infective class to a removed class and then a later return to the susceptible class. This is also true for tuberculosis, Herpes simplex and many types of influenza. Many sexually transmitted diseases (STD) such as gonorrhea and chlamydial infections are known to result in little or no acquired immunity following recovery [14]. This would mean an additional compartment $S$ in the model thus giving an SIRS model $[1,15]$. If recovery period is very short, SIS model will be the case. Obviously, the cycle of "susceptible $\rightarrow$ infected $\rightarrow$ recovery $\rightarrow$ susceptible" is an excitble cycle, which goes through "excitable $\rightarrow$ excited $\rightarrow$ refractory $\rightarrow$ excitable." This is our motivation for using excitable media to study disease spreading.

In summary, the SIR model is the most important model and all of the other models are derived from it for particular disease dynamics. The SIR model is used for the general epidemic [5], which has perfect immunization, where each individual can be infected only once. SIS model is for diseases with no immunity at all. SEIR and SEIS models are for the disease with an exposed time. SIRS model is for the disease with a temporary immunity on recovery. Among them, the SIR and SIS models are the most widely used in the literature.

All the models assume the population is homogeneous, i.e., "well mixed," and thus 
an infective individual is equally likely to spread the disease to any other member of the population or subpopulation to which it belongs $[9,10]$. So the number, size and level of interaction of the subpopulations of "susceptible," "infectious," "removed," and/or "exposed," determine the transmission of disease.

The classic models lack two effects: one is spatial spreading and the other is network topology. In the following sections, we will review the geographic spreading of diseases and disease spreading in networks. Although disease spreading in networks is also related to geographic spreading of diseases, we will separately introduce them. In the section Geographic Spread of Epidemics, we will focus on the role played by travel on the disease spreading. In the section Disease Spreading in Networks, we will focus on the role played by network topology on disease spreading.

\subsubsection{Geographic spread of epidemics}

The standard epidemic models describe diseases spreading in a large number of well mixed populations, and the emphasis is on the detailed temporal dynamics of disease transmission and control of disease. However, the spatial spread of disease is also very important and the course of infection usually can not be modeled accurately without some attention to its spatial spread. In 2003, Severe Acute Respiratory Syndrome (SARS) spread world wide from GuangZhou, a city close to Hong Kong, in a matter of weeks [16]. Every year flu viruses repeatly spread throughout the world in relatively short periods of time. In the fourteenth century, the Black Death (bubonic plague) spread from Asia throughout Europe several times [17]. All of these examples demonstrate that travel intensifies the circulation of diseases and is an important factor for the disease spreading.

To account for the geographic spread of epidemics, diffusion terms are often added to Eqs. (5.2) to generate partial differential equations (PDEs), in which $S, I$, and $R$ are functions of space as well as time $[18,19]$. Many of these models assume that 
the probability of transmission of a disease from an infective person to a susceptible person is a function of the distance between them, with the result that the epidemic diffuses out from the starting point $[18,19]$. These reaction-diffusion equations describe the spatial spread of an epidemic wave of infectiousness into a uniform population with initial homogeneous susceptibility. The conditions for the existence of epidemic traveling wave and, when it exists, its speed of propagation can be determined. The PDEs for the SIS and SIR models [18] were used to fit data available from, respectively, the historic epidemic of the Black Death of 1347-1350 in Europe and the rabies epidemics that swept through continental Europe from 1939 to 1980. However, this method treats populations as spatially continuous media, which may be a reasonable approach for describing disease spreading in situations where people are relatively stationary, but with the development of rapid forms of transportation it is necessary to consider travel, especially long-distance travel, between discrete geographical regions (cities).

Models to describe the spread of disease among several discrete groups have been presented in the literature as early as 1945, when Wilson and Worester presented results for a general deterministic epidemic model in the case of two subgroups [20]. Deterministic multiple-site modeling that includes migration as the mechanism of contact between subpopulations has been carried out by the Soviet School of mathematical epidemiology and particularly developed in the applied context of influenza prediction and control in the USSR (see the review of this work by Bailey in [10] and $[19])$.

Based on a model used by the Soviet investigators, Rvachev and Longini [21, 22] introduced a system of discrete-time difference equations in a continuous state space. It was formulated as a network of interconnected populations ( $N$ cities) connected by a transportation matrix, with elements $m_{i j}$ representing the degree of interaction between cities $i$ and $j$. It was assumed that the transportation matrix is symmetrical, i.e., $m_{i j}=m_{j i}$, and some cities were not be directly connected. It was further assumed 
that only the susceptible and the infected that are in the latent period can travel and those who are infected but isolated do not travel. This model was applied to forecast the temporal-geographic spread of the Hong Kong influenza of 1968-1969 in 52 major cities in the world, based on the transportation matrix derived from world airline statistics data in those cities in a 24-hour period, with each element $m_{i j}$ equal to the average number of individuals who traveled from city $i$ to city $j$.

Sattenspiel and Dietz [23] introduced a mathematical model that combines a model for mobility among communities with a standard SIR epidemic model. The population is divided into subgroups consisting of individuals who are assumed to be homogeneous in their mobility patterns. The model keeps track of whether and where an interaction occurs between people from different places, and it keeps track of the location of every individual of susceptible, infected, and removed (whether they are at home or visiting another community). It assumes that a particular traveler returns home before traveling to another community, and the travel rate and return rate are different. The model includes the key elements of location of a contact such that transmission probability can be made a function of location of the contact, as well as the origins of individuals involved in the interaction. In Ref. [23], a simple model for disease transmission in a population is given, with two distinct mobility patterns and a complex model to describe the 1984 measles epidemic on the Caribean island of Dominica, in which the population was divided into seven districts, each of which was further divided into three age classes. Sattenspiel et al. [24, 25] considered the same type of model but applied it to the spread of the 1918-1919 influenza epidemic in three communities of the Canadian subarctic, which can be thought of as a closed population, where travel is easily quantified. Four idealized mobility patterns, which represented idealized trade and travel relationships between different communities, were simulated and the results showed that variation in patterns of mobility significantly influences the timing of epidemic peaks but only minimally alters the number of cases within a community. The comparison of different mobility patterns indicates 
that a central location in the social and political hierarchy of a region may be more important in influencing patterns of epidemic spread than a central location with regard to travel patterns. Moreover, the simulation results showed that the travel of infected residents is the most important.

Recently, Arino and Driessche [26] formulated an SIS epidemic model with demography that described the travels of individuals between discrete geographical regions (cities), adapted from the model in Ref. [23]. The deterministic model is a system of $2 n^{2}$ ordinary differential equations for $n$ cities with terms accounting for disease transmission, recovery, birth, death, and travel between cities. They derived the basic reproduction number, which is the average number of new infectives produced by one infective introduced into a susceptible population, and gave the simulation results for travel between 2 and 3 cities.

The crucial element, however, that all such models lack is network topology. It is obvious that a given infective individual does not have equal probability of infecting all others; in the real world each individual has contact only with a small fraction of the total population, although the number of contacts that people have can vary greatly from one person to another. For most diseases, this is not an accurate representation of real contact patterns. Recently, a network of connections between individuals has been considered in the research of epidemic spreading, which will be the topic for next section. We will first review research on the effects of travel in disease spreading in networks.

A probabilistic automata network for the spread of an infectious disease in a population of moving individuals has been studied by Boccara et al. [27-29]. An automata network is a discrete dynamical system in time and space. It is a graph with a discrete variable at each vertex, which evolves in discrete time steps according to a definite rule involving the values of neighboring vertex variables [29]. A vertex is either empty or occupied by an individual belonging to one of the $S, I$, or $R$ groups. The motion of a selected individual occurs either at one of the four closest neighboring vertices (short- 
range motion) or any other vertex (long-range motion) in the graph when the vertex is empty. The model assumes that the population is closed. The results of Boccara et al. on models of SIR [27], SIS [28], and SIS with births and deaths [29] emphasized the effect of motion on the spreading of disease in one- and two-populations [27], the critical behavior between an endemic state and a disease-free state [28], and the amplitude of oscillating densities of the infective and the susceptible [29], respectively. For SIS with births, both $S$ and $I$ may give birth at a neighboring empty site to an $S$, and for SIS with deaths, both $S$ and $I$ may die, and the vertex will be empty [29]. Their results showed that as soon as the individuals start to move, the spread of the disease increases dramatically in a population. In Ref. [27], two-population models were considered, in which individuals belonging to one population may be infected only by individuals belonging to the other population, as in the case for heterosexual propagation of a venereal disease.

Recently, Miramontes and Luque [30] studied SIS epidemic models, incorporating the ideas from the dynamical small world (DSW) theory, a system of mobile automata designed to study the propagation of activity [31]. They studied the spreading of an infectious disease in a population of $N$ moving individuals, representing $S$ or $I$, in a regular lattice of $L \times L$ sites $(N<L \times L)$. At each time step the individuals move as random walkers to one of the immediate neighboring empty lattice positions, and they may move as random walkers to any empty lattice position on the lattice with probability $p$. Susceptible individuals become infected if any local neighbor is infected, and the infected individuals become susceptible after the recovery time. The numerical simulation showed that varying $p$ could modified the percolation threshold that determines whether an epidemic will prevail or not. When the system parameters are set in such a way that the initial infection has spread and the infected population is kept at a non-zero minimum level, only a small increase in $p$ caused the susceptible (healthy) population level to drop and the infected population to dominate with $p=1$. 


\subsubsection{Disease spreading in networks}

All of the classic models reviewed here have assumed that the mixing of the members of the population is homogeneous and the number of contacts per member per unit time is proportional to the total population size. It is more realistic to assume that the number of contacts per member per unit time grows less rapidly as the population size increase. It is important to look closely at mixing patterns for modeling STD diseases like AIDS, the plague of modern society, as members may have very different levels of sexual activity and the mixing may include preferences for contacts with members having a specific activity level. These aspects are intimately related to the structure of the populations, which is the focus of modeling disease spreading in recent studies.

Networks are graphs consisting of vertices (nodes) connected by edges (links, connectivities). Edges may be directed or undirected (leading to directed and undirected networks, respectively) [32]. Networks of citations of scientific papers are examples of directed networks, while networks of collaborations are undirected networks. Detailed reviews of networks can be found in Refs. [32-34]. Here, we introduce some basic terms of networks, which are helpful in understanding modeling disease spreading. The degree, $k$, of a vertex is the total number of connections, which is the same as the number of nearest neighbors of a vertex. Not all nodes in a network have the same number of edges (same node degree). The spread in the node degree is characterized by a distribution function $P(k)$ [35], which gives the probability that a randomly selected node has exactly $k$ edges, i.e., the probability for a node to be connected to $k$ other nodes. This is a basic statistical characteristic that often determines important global characteristics of networks. For example, $P(k)$ is an exponential function for small-world networks and a power law for scale-free networks. The clustering coefficient of a vertex is the ratio of existing connections over all possible connections for undirected networks. Averaging the clustering coefficients of all vertices of a network yields the clustering coefficient of the network, which reflects the "cliquishness" of 
the extent to which the nearest neighbors of a vertex are the nearest neighbors of each other [36]. Averaging shortest path length, the shortest length in unit length between two nodes, over all pairs of nodes yields the path length of the network. This quantity is often called the "diameter" of a network.

A number of authors have pursued mathematical theories of the spread of disease on networks [36-59]. All of these studies are based on standard mathematical models of SIR or SIS. To model disease spreading in networks, each node represents an individual in the state of $S, I$, or $R$, and each link represents a connection along which the infection can propagate the disease in the network. As early as 1967, Bailey $[19,44]$ used this idea to investigate disease spreading models of SIS and SIR for spatial simulations on a square lattice. Bailey considered a population of susceptibles located at the vertices, and assumed that the epidemic was started off by a single infective individual at the origin, with the infection spreading in a probabilistic way to the eight nearest neighbors. In his study, the specific cases of 121 nodes and 441 nodes were considered. However, in the real world, contact does not occur only at nearest neighbors, as we reviewed in the last section. In addition, the real world is not in the order of a regular lattice. The most widely used networks in recent studies are small-world networks and scale-free networks.

The small-world model was first proposed by Watts and Strogatz (WS) [36] to mimic properties of social networks. The model is defined as follows: a ring with $N$ nodes is defined, in which each node is symmetrically connected to its two nearest and next-nearest neighbors. For every node, each link connected to a clockwise neighbor is rewired to a randomly chosen node with probability $p$, and preserved with probability $1-p$. The long range connections generated by this process decrease the distance between the vertices, leading to the small-world phenomenon [60,61], often referred to as six degrees of separation [62]. $p$ characterizes the degree of disorder of the network, ranging from a regular lattice to a completely random graph, respectively, with $p$ running from 0 to 1 . Figure 5.1 [36] shows a regular ring lattice, a small-world 


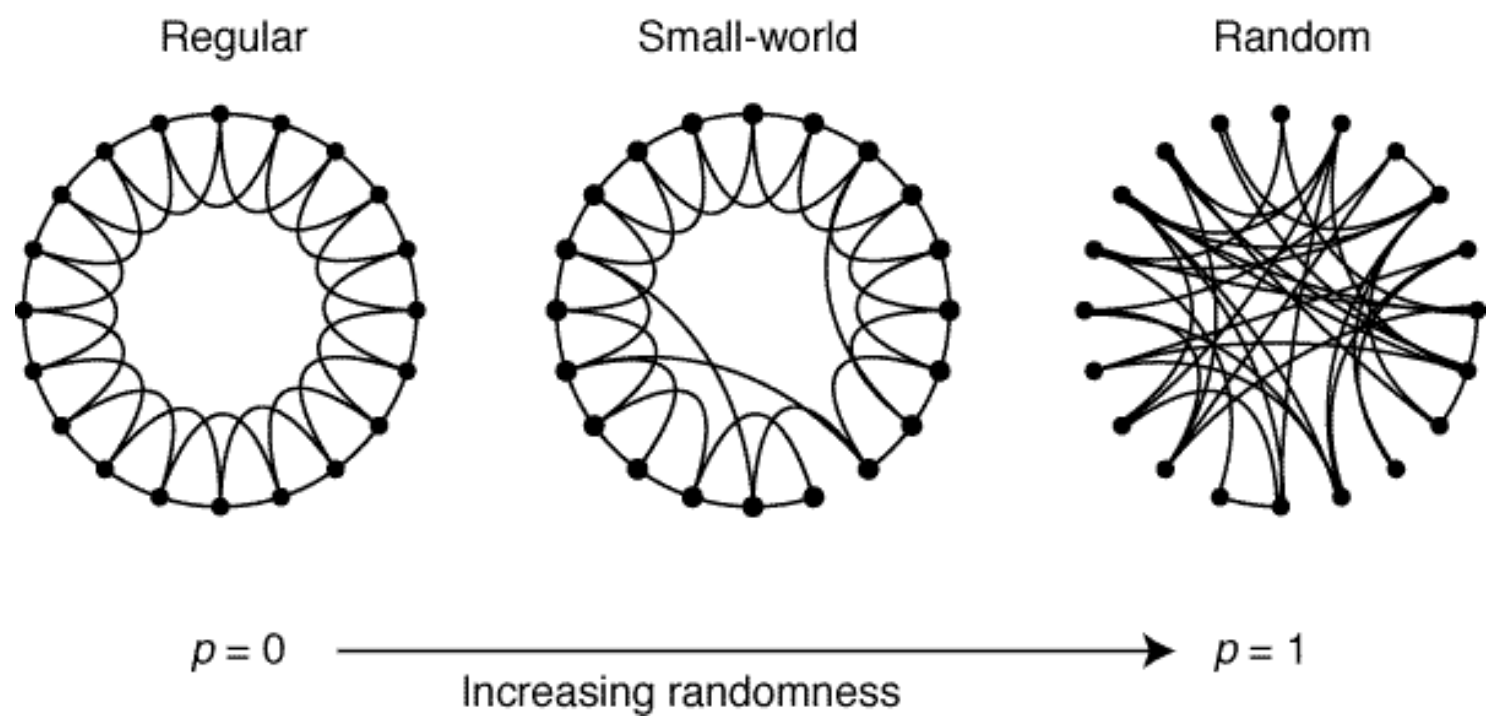

Figure 5.1. "Small world" between a regular ring lattice and a random network, with $p$ changing from 0 to 1 , without altering the number of vertices or edges in the graph. Twenty nodes with a node degree of four are shown here. (Adapted from [35].) 
lattice, and a random network. The WS small-world model has been modified by Newman and Watts [50] in such a way that a low density of "shortcuts" are added between randomly chosen pairs of sites while preserving the original connections in a regular one- and two-dimensional lattice.

Watts and Strogatz [36,37] used an SIR model to investigate the spread of infectious disease in their small-world model. Initially, one single infective individual is introduced into the susceptible populations. The infected individual can infect each of its susceptible neighbors with a infection rate ranging between 0 and 1 . They found that the time for the disease to infect half of the population decreased rapidly for a small increase in rewiring probability $p$.

Kuperman and Abramson [39] studied the WS small-world effect in an SIRS model. The interactions within the population were described by a one-dimensional WS small-world network. In their simulations, infected populations were initially distributed randomly and the rest were susceptible. A susceptible would become infected with a certain probability when any of its neighbors were infected. Each element went through the cycle of "susceptible $\rightarrow$ infected $\rightarrow$ recovery $\rightarrow$ susceptible." They observed that the temporal dynamical behavior of the model changed from an endemic situation at small $p$ to a spontaneous state of large-amplitude oscillations at large $p$. Here, $p$ is the parameter indicting small-world [36] behavior. In Ref. [43], the authors introduced a modified version of the SIRS model by including a ring vaccination program for the outbreaks of foot-and-mouth disease (FMD) on small-world networks. In this article, the authors claimed that the small-world network is better than regular or random lattices in modeling the outbreaks of FMD, since the small-world network describes both local and non-local interactions.

The behavior of epidemiological percolation models on variants of the WS smallworld model has also been investigated $[42,47,48,50]$. An epidemiological percolation model can be described as follows: each site of a lattice represents the location of an individual. A site can infect its neighbors if itself infected. With the probability, 
$p$, some sites are immune. This is called the site percolation model. Hence, if bonds (representing contacts between neighbors) are present with probability $p$ (some transmissions possible, some forbidden), then we have a bond percolation model [47]. As Newmal et al. [48] stated, "bond percolation is equivalent to the standard SIR model of disease spread with the percolation threshold mapping to the epidemic threshold of the disease in the SIR model, and cluster sizes mapping to the sizes of disease outbreaks which start with a single disease carrier." For the site percolation model, the same "mapping" should also be valid.

Newman and Watts [50] used a variant of the WS small-world model as a simple model of disease propagation. They suggested using a site percolation model for disease spreading in which some fraction of the populations are considered to be susceptible to the disease. They gave a simple differential equation model for disease propagation on an infinite graph in which communication of the disease takes place with $100 \%$ efficiency, i.e., all susceptible acquaintances (neighbors) of an infected person become infected. They obtained an approximate solution for the threshold fraction of susceptible individuals for epidemics as a function of the density of shortcuts on the lattice. Moore and Newman [42] used a variant of the one-dimensional small-world graph, in which transmission between individuals takes place with less than $100 \%$ efficiency, and derived an exact solution of the percolation threshold (i.e., the epidemic threshold) for both the site percolation model and the bond percolation model.

Newman et al. [48] proposed a model of bond percolation on two-dimensional small-world networks to describe the spread of plant diseases. Their analytical solution of the model gives accurate predictions for quantities such as the position of the percolation threshold and the typical size of disease outbreaks as a function of the density of "shortcuts" in the small-world network. Sander et al. [47] studied bond percolation for the spread of disease with an SIR model that includes variations in the susceptibility to infection. The variations were realized by changing the 
bond strengths that indicate the efficiency of disease transmission from the infected to the susceptible. The results show that with strong heterogeneity, patchiness in the spread of the epidemic is very likely, and the criterion for epidemic outbreak depends strongly on the heterogeneity. The results of weak heterogeneity do not differ very much from the case with homogeneity.

In contrast to small-world networks, in which new connections ("shortcuts") between vertices are chosen without any preference and the degree distribution is exponential, scale-free networks exist with preferentially linking, and the degree distribution follows a power law [63]. Barabàsi and Albert [63] first found a common property of many large networks in which the vertex connectivities follow a scale-free power law distribution. They introduced a graph, referred to as the BA graph, as a model of a growing network in which the successively added nodes establish links with higher probability, pointing to already highly connected nodes [63]. It is constructed using the following algorithm: starting from a small number $m_{0}$ of nodes, at every time step a new vertex is added, with $m\left(m<m_{0}\right)$ edges that link the new vertex to $m$ different vertices already present in the system. It was assumed that the probability that a new vertex will be connected to a vertex (target node) depends on the connectivity of that vertex in a form of a preference function that was defined. After iterating $t$ times, a network composed of $N=t+m_{0}$ nodes and $m t$ edges is obtained. This network evolves into a scale-invariant state with a connectivity distribution $P(k) \sim k^{-\gamma},(\gamma=2.9 \pm 0.1)$. Figure 5.2 [34] shows a scale-free network, consisting of 200 nodes, where the preference function is that the probability of attachment is proportional to the degree of the target node. It has been demonstrated that the BA algorithm is not a unique model generating scale-free networks [64], and a modification of the BA model is changing the form of the preference function $[32,51,65]$ and the value of $\gamma$, usually $2<\gamma \leq 3$. SF networks find real examples [32] in several technological systems such as the Internet $[66,67](\gamma=2.3)$ and the world-wide-web (WWW) [68] $(\gamma=2.1)$, as well as the web of human sexual contacts $(\gamma=3.4)[69]$. 


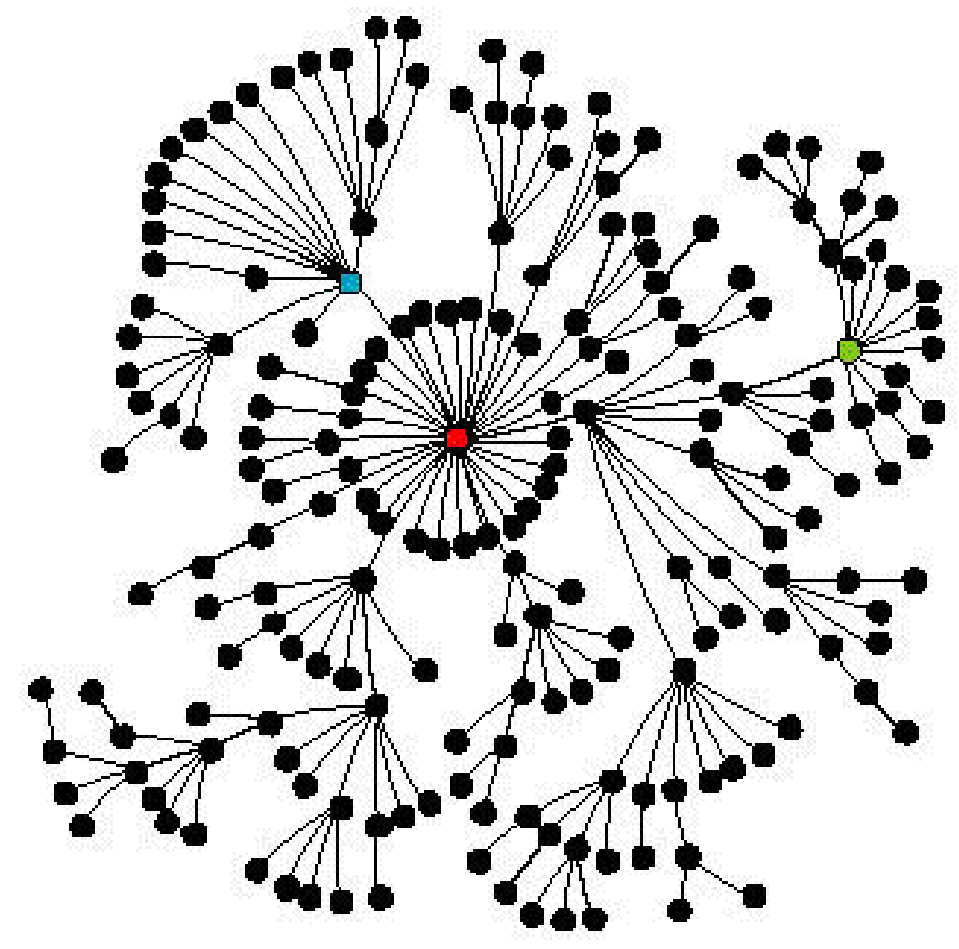

Figure 5.2. Scale-free graph, grown by attaching new nodes at random to previously existing nodes. The probability of attachment is proportional to the degree of the target node; thus richly connected nodes tend to get richer, leading to the formation of hubs and a skewed degree distribution with a heavy tail. Colours indicate the three nodes with the most links (red, $\mathrm{k}=33$ links; blue, $\mathrm{k}=12$; green, $\mathrm{k}=11$ ). (Adapted from [33].) 
An important step in understanding epidemic spreading in SF networks has been made by Pastor-Satorras and Vespignani $[38,52]$. They analyzed real data from computer virus infections using the SIS epidemic model defined in BA scale-free graphs. In striking contrast with the usual models for the spread of infection in human and other populations, they found no threshold for epidemic spreading. Within the observed topology of the internet and WWW, viruses can spread even when infection probabilities are vanishingly small. They also found that, in its early phase, the epidemic spreads relatively slowly and non-exponentially, again in contrast with the initial exponential behavior in conventional epidemics. May and Lloyd [53] reported a comprehensive study of the SIR model, which is argued to be a more appropriate model for computer viruses, in scale-free networks, which extends the preliminary account provided in [49]. They pointed out that the reason of the absence of a threshold in the SIS model for the spread of infection is the extreme heterogeneity in the connectivity distribution of a scale-free network, i.e., the infinite size of the network [32]. They demonstrated that networks of finite size do exhibit threshold effects.

Recently, the problem of the absence of an epidemic threshold in SF networks prompted a number of mathematical studies $[51,54-56,58]$ of the spread of disease on SF networks. These studies pursued constructing SF networks with different ranges of values of $\gamma[51,54]$ from the BA graph or different preferential functions [55]. Volchenkov et al. [51] showed that the epidemic spreading in scale-free network is very sensitive to the statistics of degree distribution characterized by $\gamma$, the effective spreading rate, the preferential linking function, and the immunization of infected nodes.

Moreno and Vàzquez [54] studied the dynamics of SIS and SIR models for infectious disease in structured scale-free networks generated with the deactivation model algorithm [65], which makes the power law connectivity distribution of the structured scale-free networks have a quite wide interval of $\gamma(2 \leq \gamma \leq 4)$, and the graph lacks small-world properties. They showed that the existence of an epidemic threshold for 
the SIS model in this particular SF network depends on the initial density of infected individuals when the connectivity fluctuations of the network are unbounded. The epidemic threshold for the SIR model exists in the network they constructed. Boguña et al. [55] analyzed the conditions for the lack of an epidemic threshold in the SIS model in unstructured, undirected SF networks to establishing the general conditions for the existence of an epidemic threshold. In unstructured undirected networks all vertices within a given degree class can be considered statistically equivalent. They are different from structured networks in which the distance or time ordering can be defined $[56,58]$, like small-world networks. They found that an SF network $(2<\gamma \leq 3)$ with a two-point correlation is a sufficient condition for a null epidemic threshold in unstructured networks with assortative or disassortative mixing. "Assortative mixing" means that vertices with a high degree will connect preferably to highly connected vertices, and "disassortative mixing" means that highly connected vertices are preferably connected to vertices with low degree. These characteristics can be defined in terms of the conditional probability $P\left(k^{\prime} \mid k\right)$, where a node of connectivity $k$ is connected to a node of connectivity $k^{\prime}[56,58]$.

\subsection{Modeling Disease Spreading with Excitable Me- dia}

\subsubsection{Overview}

Our study is a description of epidemic spreading based on excitable media, which is unique among all the studies we have reviewed. We consider the contagious diseases that are transmitted exclusively by direct contact. Once people are infected, they will lose their immunity and after recovery they will be susceptible again. Such kinds of disease include pertussis (whooping cough or chooping cough), Herpes simplex, tuberculosis, some STDs such as gonorrhea and chlamydial infections, and many 
types of influenza. These diseases can be modeled by the SIRS model. The three states of epidemic disease, the susceptible state, the infected state, and the recovery state, are analogous to the three states, the excitable state, the excited state, and the refractory state, respectively, of excitable systems such as the BZ reaction.

Reaction-diffusion systems [18] have been used in modeling geographical disease spreading. A reaction-diffusion chemical system is ideal for modeling disease spreading, as the chemical wave models nearest neighbor contacts. In the real world, some diseases are transmitted from others who are neither neighbors nor family members in immediate contact. We can mimic long-distance contact, analogous to a shortcut in the small-world model, by using a video-projector method for the experiments. Combining the local contact with long distance contact is the most important part of our study of modeling disease spreading in excitable media.

In our study, the photosensitive BZ reaction was used to investigate the spatiotemporal dynamics of epidemic disease spreading in a spatially extended medium. The propagation of excitation chemical waves in an excitable BZ system mimic the spreading of disease. We construct a two-dimensional square network $(\mathrm{N} \times \mathrm{N}$ elements $)$ consisting of an array of square cells, each representing a subpopulation of the network. In addition to the local interactions (diffusive) between cells, a number of long-distance interactions (non-diffusive, non-local) are added over the network and all the original local interactions are preserved, as in the modified small-world model [50]. Three different realizations of long-distance interaction have been studied, namely static links, dynamic links, and the domain model. We now summarize the common aspects of the experimental and numerical studies before we introduce the three different realizations. 


\section{Experimental study}

Experiments were carried out with ruthenium(II)-bipyridyl $\left(\mathrm{Ru}(\mathrm{bpy})_{3}^{2+}\right)$, a light sensitive catalyst of the BZ reaction, immobilized in a thin slab of silica gel, which was cast onto a microscope slide and placed in a thermostated reactor that was continuously fed with fresh, catalyst-free BZ solution to maintain constant, non-equilibrium conditions. The experimental setup is the same as in the study of "Synchronization of Spatiotemporal Patterns in Locally Coupled Excitable Media," described in Section 4.2.4 and shown in Fig. 4.1. The reaction was carried out at $20.0^{\circ} \mathrm{C}$ with catalyst-free BZ solution maintained at $0^{\circ} \mathrm{C}$ to prevent decomposition and degassed before pumping into the reactor. Composition of the catalyst-free BZ reaction mixture was 0.552 $\mathrm{M} \mathrm{NaBrO}_{3}, 0.026 \mathrm{M}$ malonic acid, 0.162 M bromomalonic acid, and $0.489 \mathrm{M} \mathrm{H}_{2} \mathrm{SO}_{4}$. The silica gel was prepared by acidifying a solution of $10 \%$ (w/w) $\mathrm{Na}_{2} \mathrm{SiO}_{3}$ and 2.05 $\mathrm{mM} \mathrm{Ru}(\mathrm{bpy})_{3}^{2+}$ with $\mathrm{H}_{2} \mathrm{SO}_{4}$ and casting a uniform $0.3 \mathrm{~mm} \times 37 \mathrm{~mm} \times 37 \mathrm{~mm}$ layer onto a microscope slide. The intensity of the projected image was adjusted at each pixel by an iterative algorithm to ensure spatial homogeneity of the illumination field. The medium was illuminated with light (intensity $0.310 \mathrm{~mW} / \mathrm{cm}^{2}$ ) from a modified video projector passing through a $460 \mathrm{~nm}$ bandpass filter and was monitored with a CCD video camera. A $36 \mathrm{~mm} \times 36 \mathrm{~mm}$ image produced by the computer-controlled video projector was projected onto the face of the gel medium. Bromide ion is produced in a photochemical cycle when the ruthenium catalyzed BZ reaction is exposed to $460 \mathrm{~nm}$ light, which inhibits the autocatalysis of the reaction. The excitability of the system can therefore be adjusted by varying the light intensity.

The region in the illumination field consists of $33 \times 33$ cells, with each cell composed of $12 \times 12$ pixels, and a total area of $36 \times 36 \mathrm{~mm}^{2}$. At the beginning of an experiment, a circular wave was initiated in the center of the medium to mimic the initialization of the disease. The presence of the chemical wave in each cell was monitored with the video camera. If the chemical wave occupation area in a cell was over a coverage threshold, of which $50 \%$ was used, the cell was defined as an infected cell. 
In other words, if $50 \%$ of the elements in a cell are excited as $\mathrm{Ru}(\mathrm{bpy})_{3}^{3+}$, this cell will be defined as an infected cell. Each infected cell has a probability of interacting with a cell which is not near it, which mimics long-distance interactions. How the infected cell "travels" and where it travels to will depend on different strategies, which will be explained below. The cell where the long distance interaction occurs was maintained with a light intensity of zero for a period of time (1.0 min) until it became oscillatory in order to initiate a new chemical wave. This mimics disease transmission by long-distance travel. Chemical waves were monitored at equal-time intervals (6.0 s) for every cell. The process of finding infected cells and adding the long-distance interaction was updated every $6.0 \mathrm{~s}$. The fraction of the medium in the excited state was calculated in the process of the experiment. The first coverage time $\tau$, which is defined as the time it takes for each of the cells to be infected at least once, was also recorded in the process of the experiment.

\section{Numerical simulations}

Numerical simulations were carried out using the modified Oregonator model [7073], which includes the additional term $\phi(t)$, taking into account the photochemical production of bromide:

$$
\begin{aligned}
\frac{\partial u}{\partial t} & =D_{u} \nabla^{2} u+\frac{1}{\varepsilon}\left\{u-u^{2}-[f v+\phi(t)] \frac{u-q}{u+q}\right\} \\
\frac{\partial v}{\partial t} & =u-v
\end{aligned}
$$

where the variables $u$ and $v$ correspond to the dimensionless concentrations of the autocatalytic species $\mathrm{HBrO}_{2}$ and the oxidized catalyst $\left(\mathrm{Ru}(\mathrm{bpy})_{3}^{3+}\right), \varepsilon$ and $q$ are scaling parameters, and $f$ is an adjustable stoichiometry parameter. The rate of bromide production from irradiation, $\phi_{i}(t)$, is proportional to the light intensity. The parameters to maintain an excitable media are: $\epsilon=0.01, q=0.002, f=1.4, \phi=0.073, D_{u}=1.0$. 
The modified Oregonator was integrated in a two-dimensional lattice of $1000 \times$ 1000 grid points by an explicit Euler method (unit grid size $\Delta x=0.15$, time step $\Delta t$ $=0.001)$ with non-flux boundary conditions and a five-point approximation of the Laplacian. The medium consists of $50 \times 50$ cells with each cell of $20 \times 20$ pixels and a total area of $150 \times 150$ dimensionless space units. As in the experimental study, if the chemical wave occupation area in a cell was over a coverage threshold, of which $50 \%$ was used, the cell was defined as an infected cell. The time interval was 100 time steps for maintaining a chosen cell for a non-diffusive interaction at a light intensity of zero in order to initiate a new chemical wave. The process of monitoring chemical waves and detecting infected cells as well as adding the long distance interactions were updated every time step. The fraction of the medium in the excited state and the first coverage time were calculated in the process of the simulations.

\subsubsection{Static networks}

In our system, static links between the "source cell" and the "destination cell" mimic non-diffusive jumps. The non-diffusive interaction is realized in such a way that once a "source cell" is infected, it will infect its "destination cell." The number of static links determines the number of pairs of "source cells" and "destination cells." Static links mimic long-distance travel that always occurs to the same place. Work related travel for adults and school events for students are examples of static links.

The rule for choosing "source cells" and their "destination cells" is the following: the "source cell" was chosen randomly and its "destination cell" was chosen randomly among the total number of cells in the network. The source cell is not allowed to be identical to the destination cell, i.e., a source cell can not be the destination cell for itself. However, a "source cell" can have multiple "destination cells," and one "destination cell" can have multiple "source cells," since a cell is chosen randomly in the whole network and a cell may be selected more than once. Hence, the more 
static links, the greater the chance to have multiple "destination cells" for one "source cell" and multiple "source cells" for one "destination cell." Figures 5.3(a)-5.3(c) show the "source cells" and "destination cells" for 10, 100, and 500 links respectively, in a network of $50 \times 50$ cells. All of the possible long-distance travel is represented by the arrows. The origins of the arrows are the "source cells" and the ends of the arrows are the "destination cells."

All elements of the network are initially equally susceptible to the disease. Pairs of "source cells" and "destination cells" were chosen as described above. A circular chemical wave was initiated in the center of the medium by a black square. Figure 5.4 shows an example image from an experiment when black initiation zones were imposed onto the gel at the "destination cells" when the static links were equal to 100. The images in Figs. 5.5(a) and 5.5(b) show the time sequence of chemical wave propagation with 100 and 300 static links, respectively, in the experiment. The periodic behavior of wave generation in these figures clearly shows that once a "source cell" is infected the "destination cell" is infected. Figures 5.6(a)-5.8(a) show the time series of the fraction of excited elements in the system for different numbers of static links in the experiment. Figures 5.6(b)-5.8(b) show the power spectra of the corresponding time series. We see that the frequency at 0.02 is more pronounced when the number of links is increased.

The first coverage time for different static links were normalized by the first coverage time with the number of static links set to zero. The relationship between the normalized first coverage time and different probabilities is given by a power law in a $\log -\log$ plot, as shown in the Fig. 5.9. Here, the probability is defined as the ratio of the number of static links over the total number of possible links. For example, in our experimental study, the total number of cells is $1089(33 \times 33)$ and the total number of possible links is given by $1089 \times(1089-1)$. So the probability is $8.44 \times 10^{-5}$ for 100 static links.

In the numerical simulations, we obtain similar results as in the experiment. Figure 


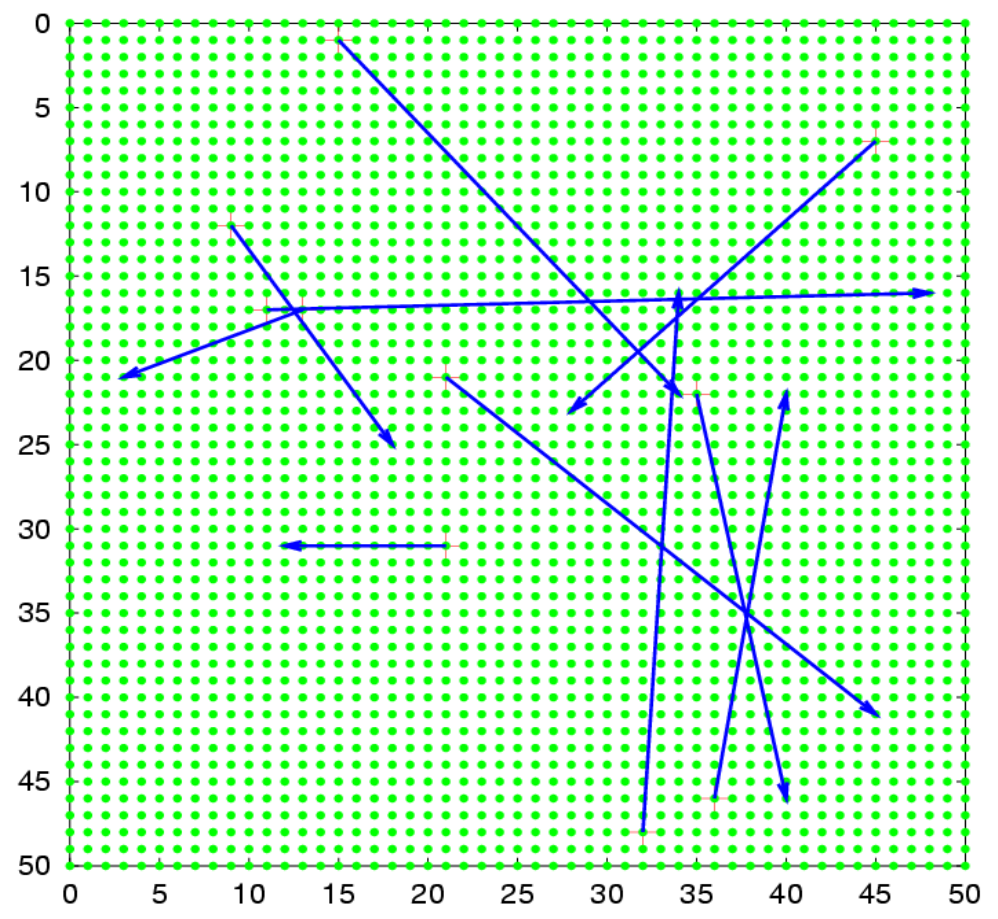

Figure 5.3(a). Static networks with 10 links. 


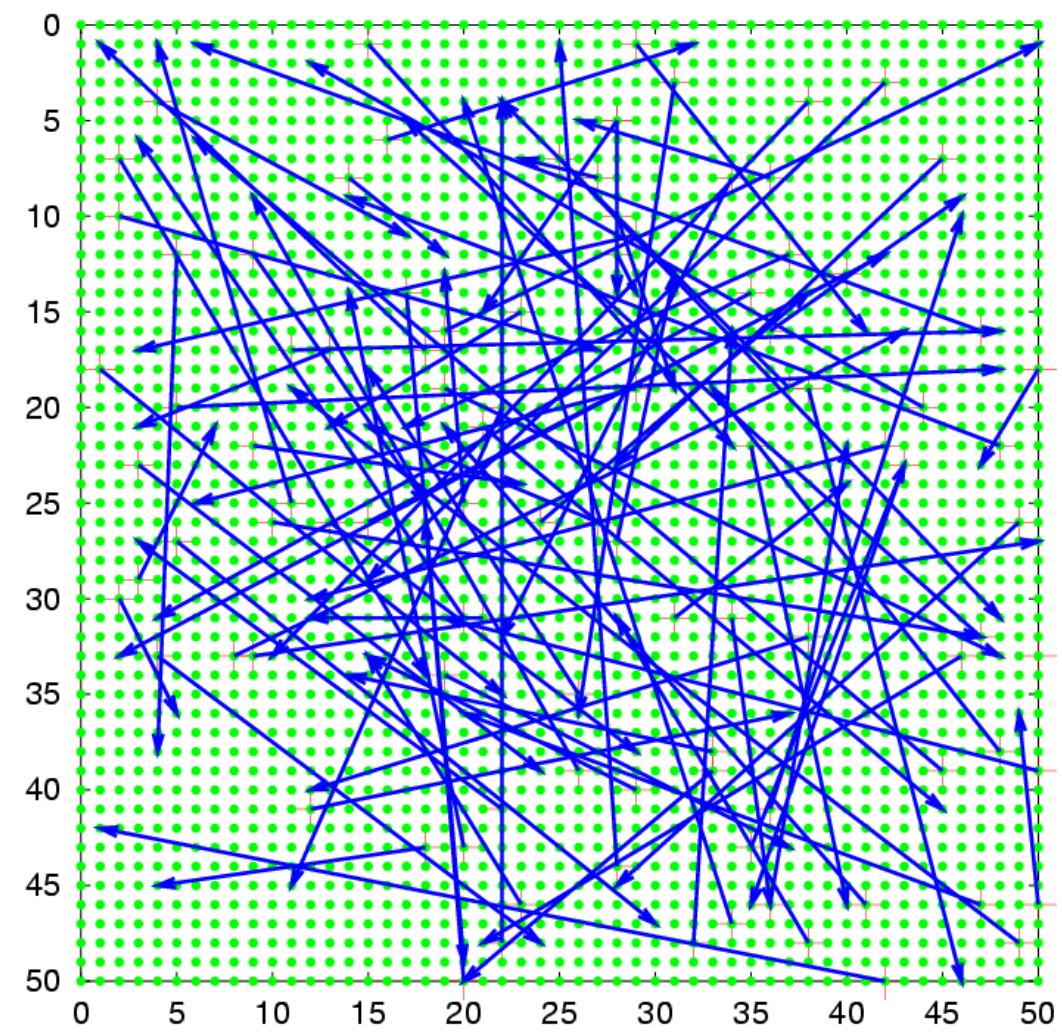

Figure 5.3(b). Static networks with 100 links. 


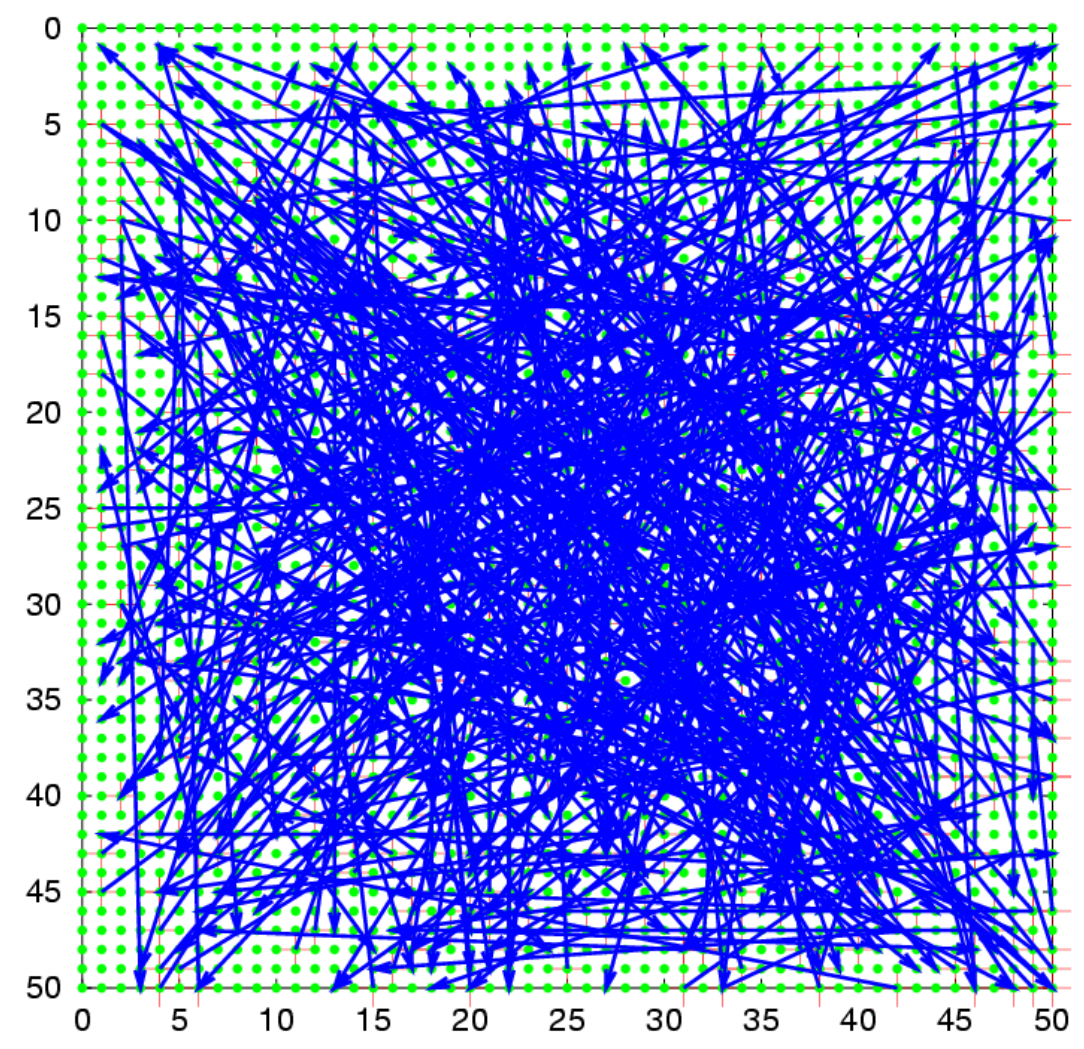

Figure 5.3(c). Static networks with 500 links. 


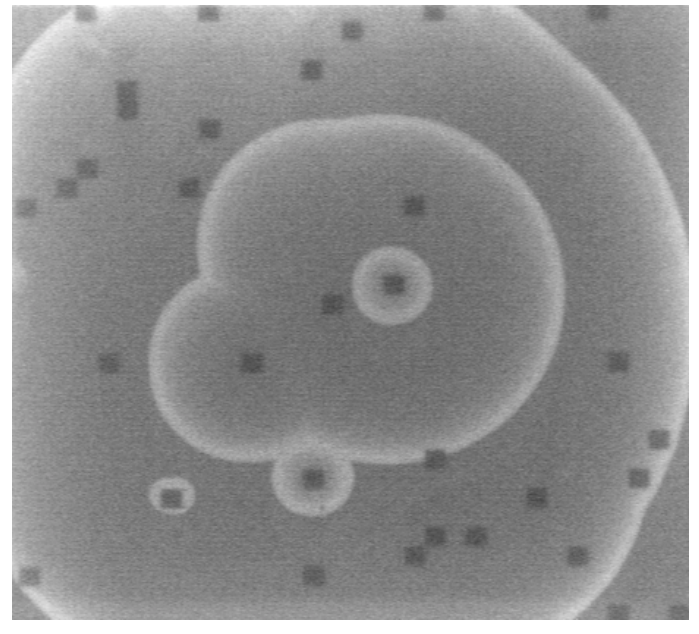

Figure 5.4. Chemical wave in the static network system with the imposed long-distance interactions. 


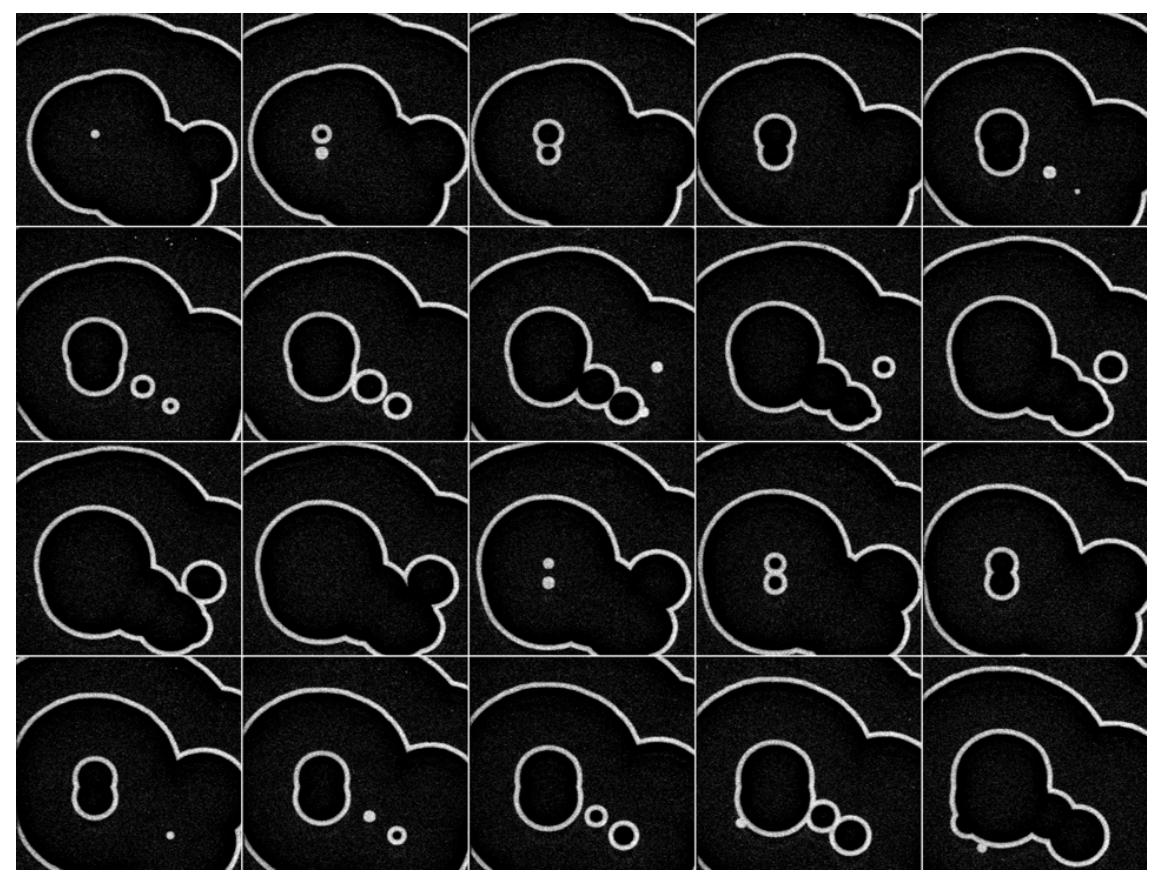

Figure 5.5(a). Experimental results for 100 static links. Images showing time sequences of chemical wave propagation with static links in the excitable BZ medium (time increases from left to right and then to the next row). The time interval between each frame is $6.0 \mathrm{~s}$. The gray level in each panel is proportional to the concentration of the catalyst, $\mathrm{Ru}(\mathrm{bpy})_{3}^{3+}$, with white and black representing high and low values, respectively. The region in the illumination field consists of $33 \times 33$ cells, with each cell of $12 \times 12$ pixels, and a total area of $36 \mathrm{~mm} \times 36 \mathrm{~mm}$. Every infected "source cell" jumps to its "destination cell." The jump was updated immediately after the wave behavior was monitored every $6.0 \mathrm{~s}$, and each destination cell was maintained at zero intensity for $1.0 \mathrm{~min}$. 


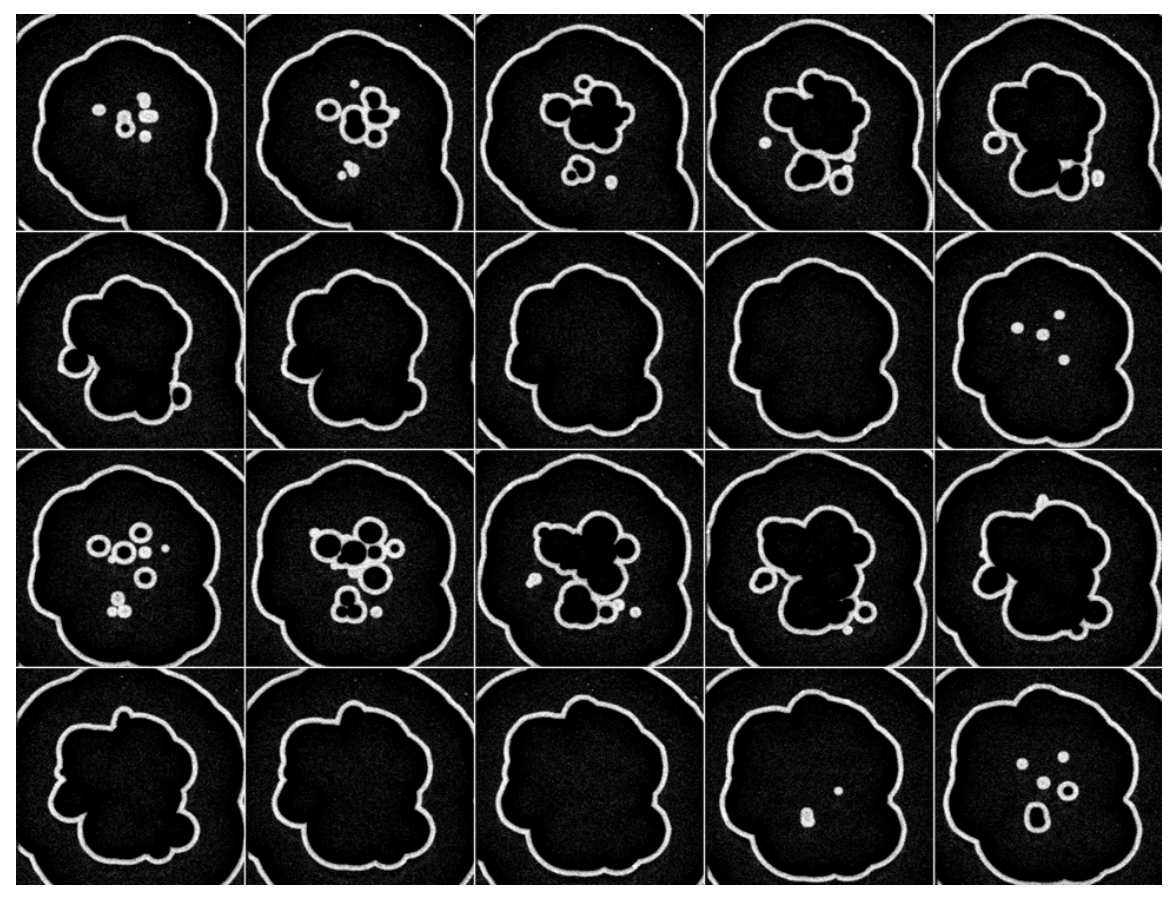

Figure 5.5(b). Experimental results for 300 links. All other conditions as in Fig. 5.5(a). 


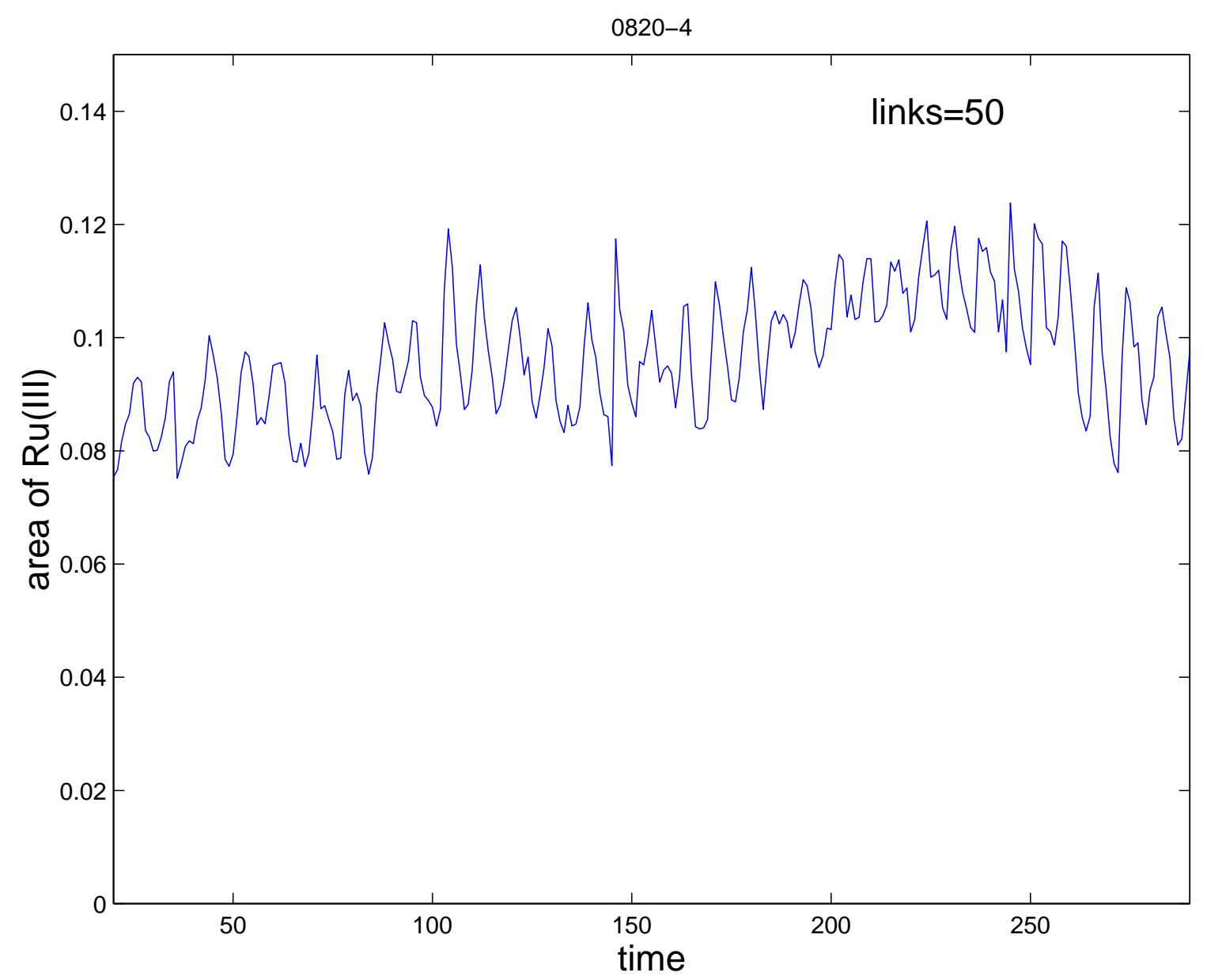

Figure 5.6(a). Time-series of fraction of infected elements in the experiment. Area of $\mathrm{Ru}(\mathrm{III})$, which represents the fraction of the area of the oxidized catalyst, $\mathrm{Ru}(\mathrm{bpy})_{3}^{3+}$, in the medium, represents the fraction of infected elements. Time is represented by the frame number. The time interval between each frame is $6.0 \mathrm{~s}$. The number of static links is 50 . Other conditions as in Fig. 5.5(a). 


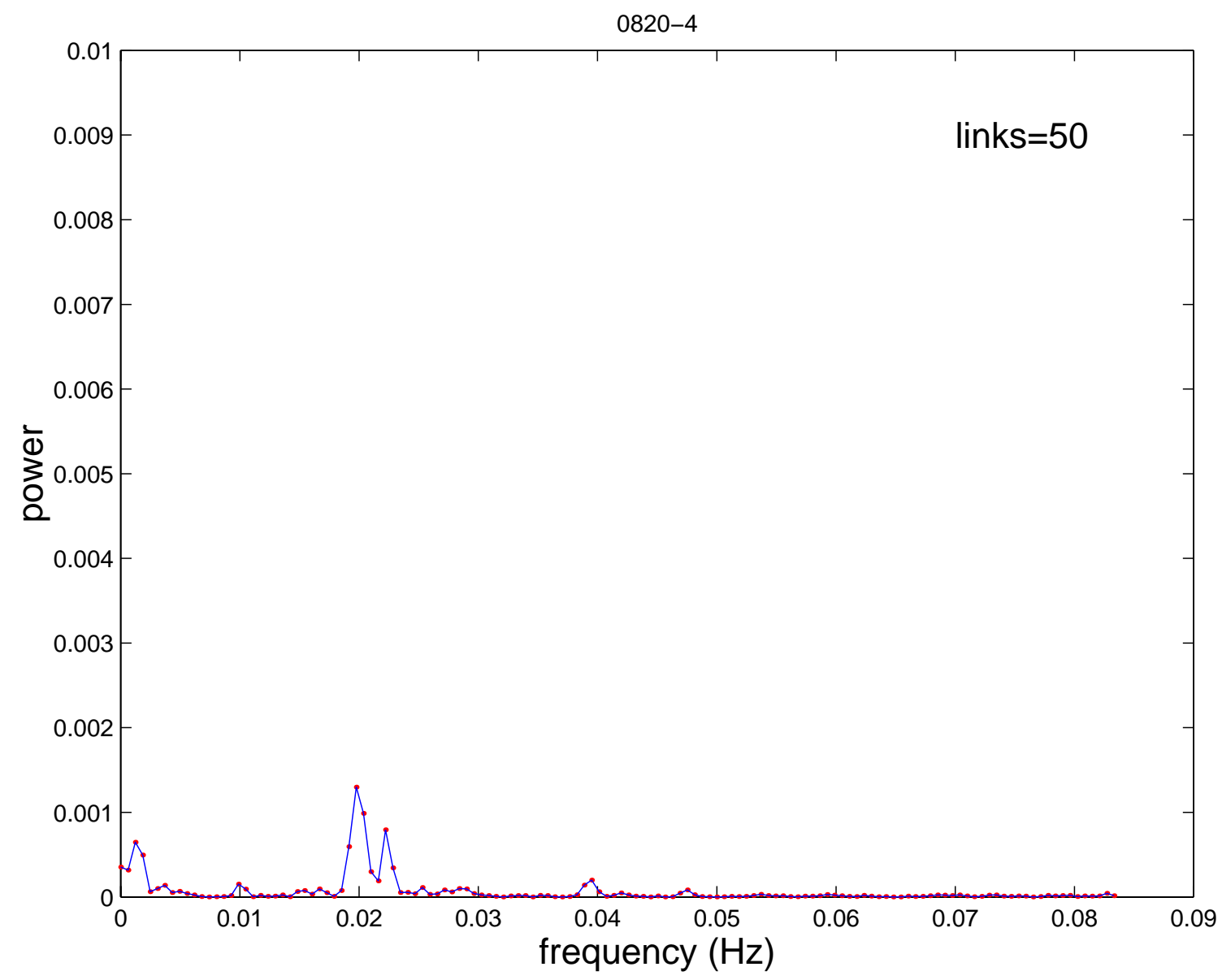

Figure 5.6(b). Power spectrum of the time-series of fraction of infected elements in the experiment for 50 static links. 


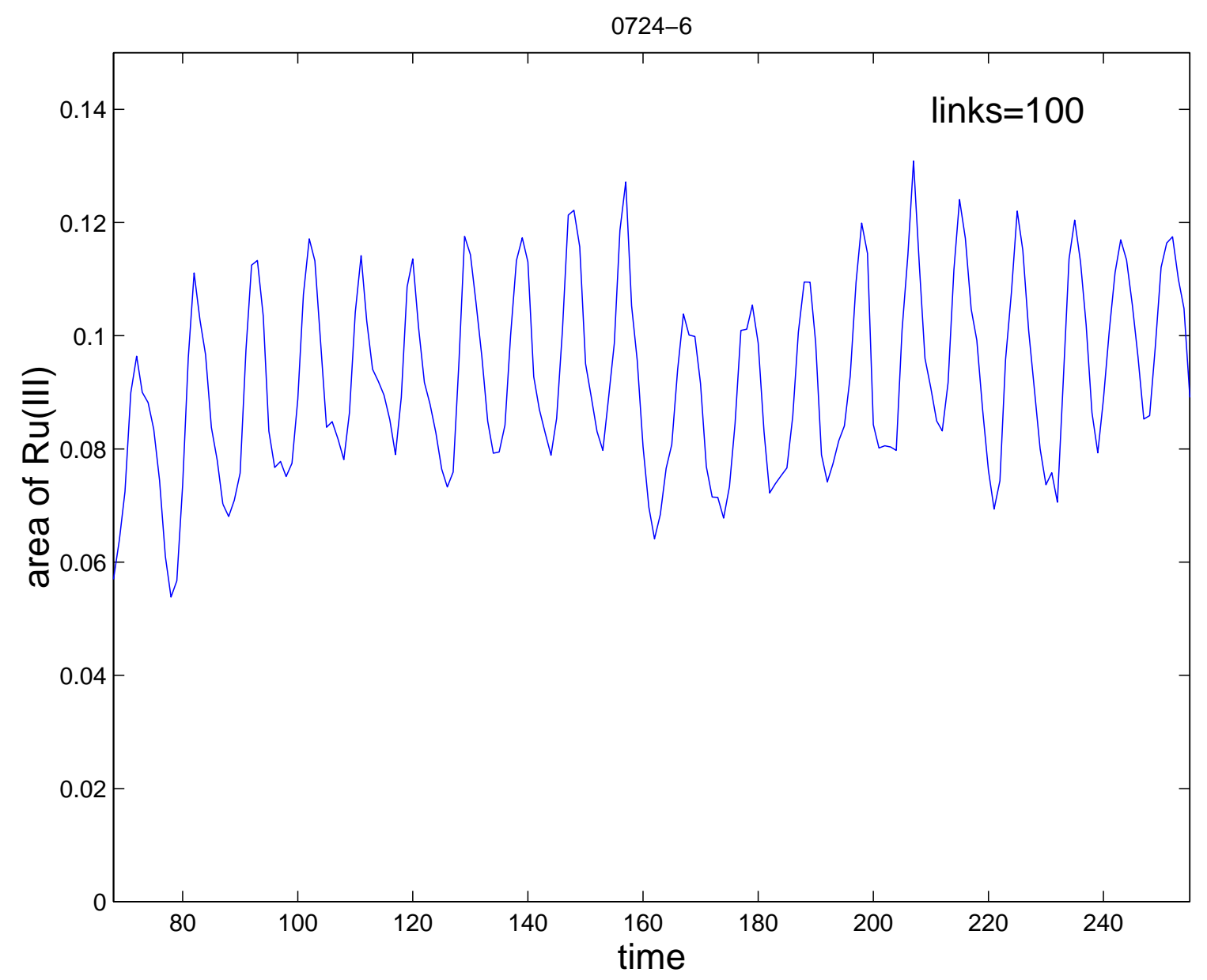

Figure 5.7(a). Time-series of fraction of infected elements in the experiment for 100 static links. Other conditions as in Fig. 5.6(a). 
$0724-6$

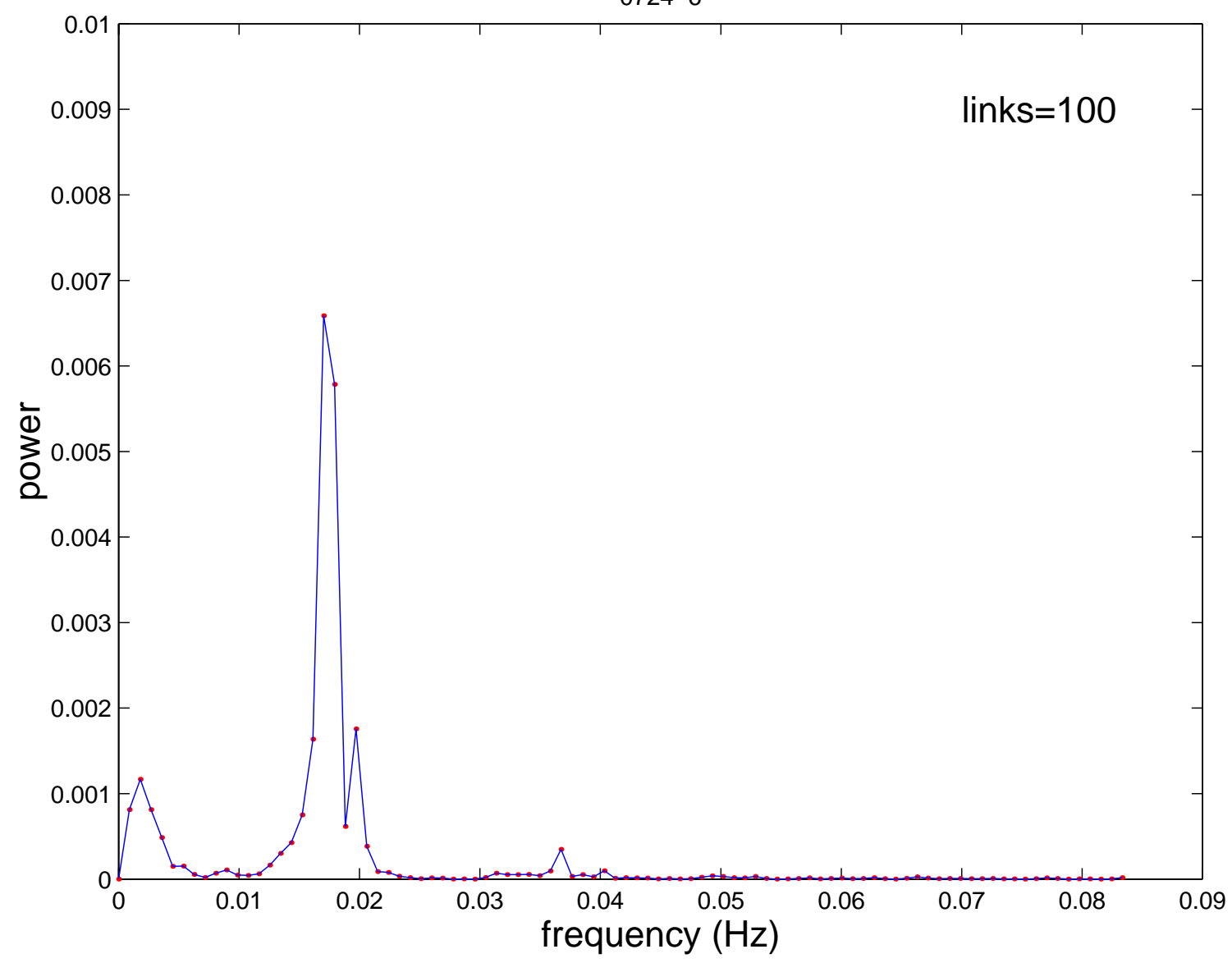

Figure 5.7(b). Power spectrum of the time-series of fraction of infected elements in the experiment for 100 static links. 


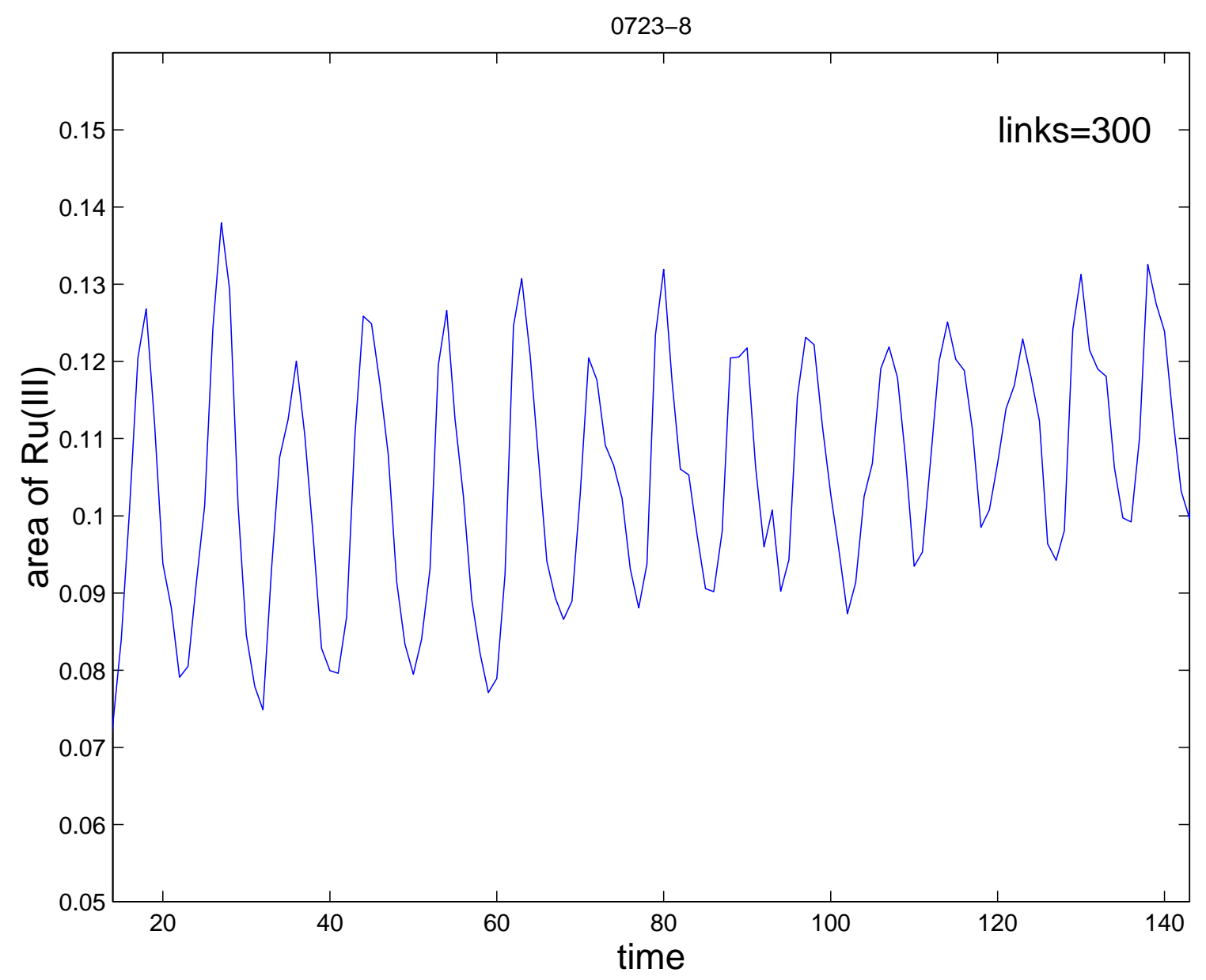

Figure 5.8(a). Time-series of fraction of infected elements in the experiment for 300 static links. Other conditions as in Fig. 5.6(a). 
0723-8

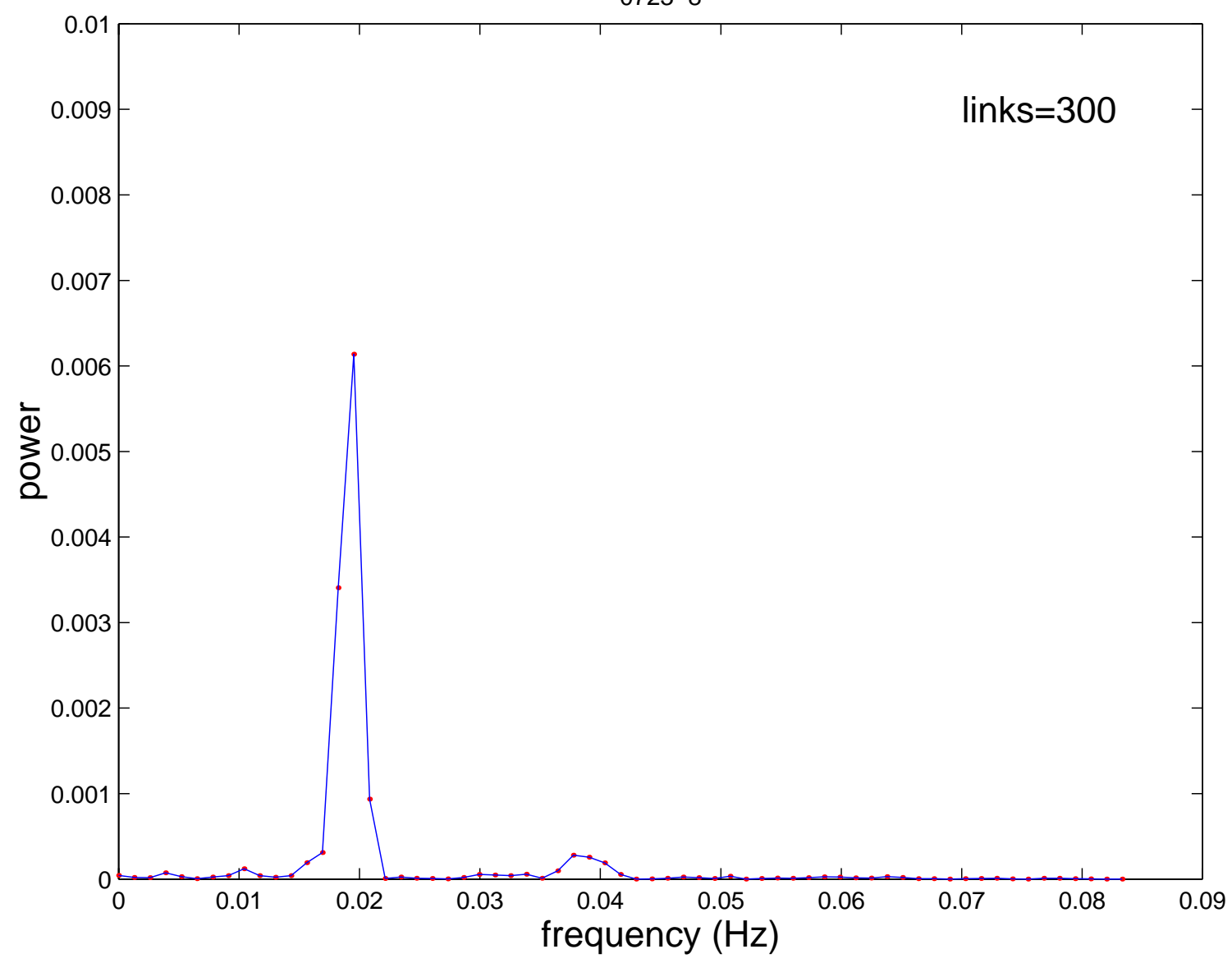

Figure 5.8(b). Power spectrum of the time-series of fraction of infected elements in the experiment for 300 static links. 


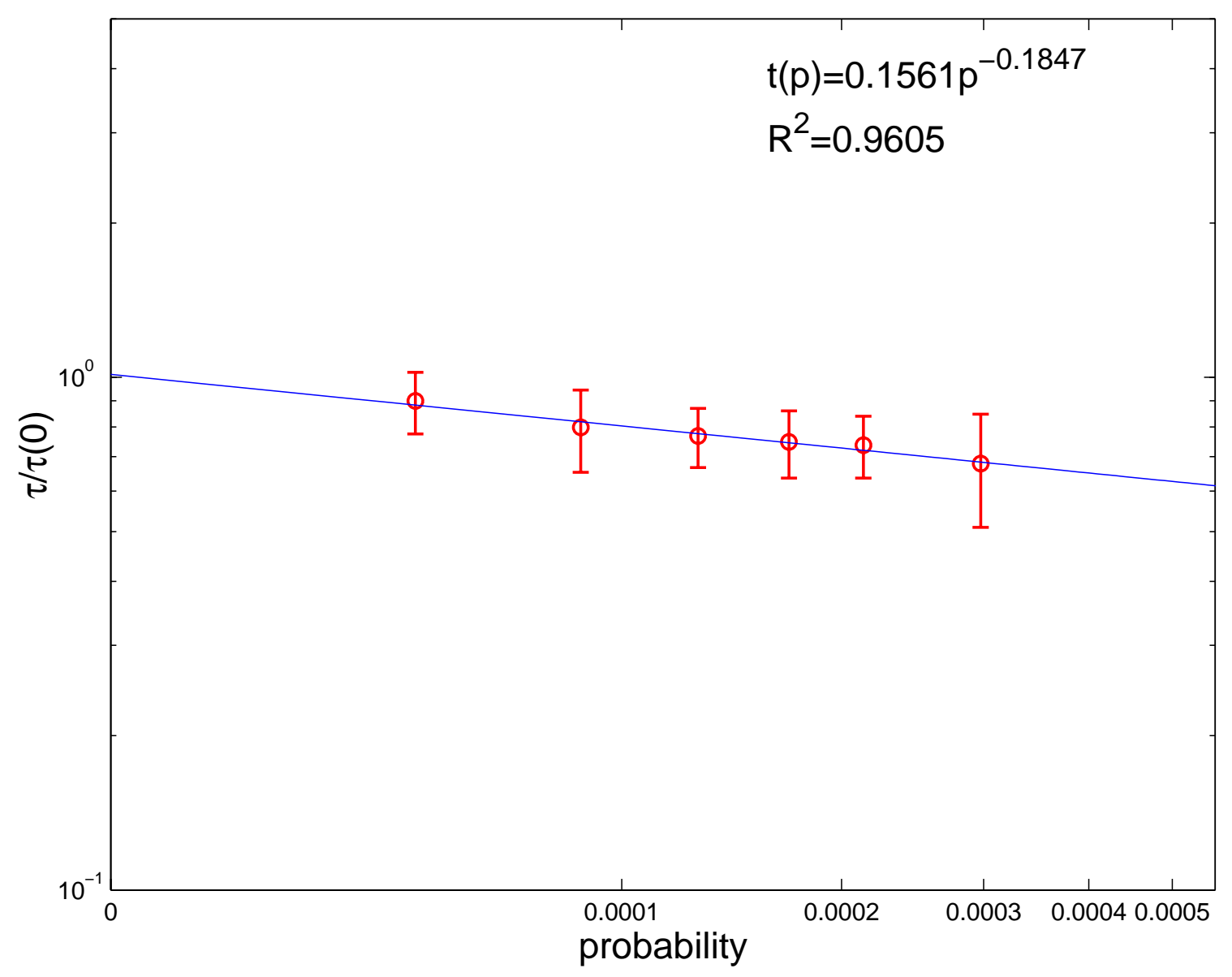

Figure 5.9. Experimental results of normalized coverage time, $\tau / \tau(0)$, as a function of probability, $p . p$ is the ratio of staic links over the total possible links. $\tau$ is the time for infecting each cell at least once. $\tau(0)$ is the time of one circular wave propagating from the center to cover the entire medium with 0 links. The solid blue line is given by the power law $\tau / \tau(0)=0.156 p^{-0.1847}$. The red circles show mean values from 5-6 measurements and bars indicate standard deviations. 
5.10 shows the time series of fraction of excited elements for different numbers of static links in the numerical simulations. In the range from 10 to 450, we observe sustained oscillations of infection in the whole medium, and for larger numbers of static links the sustained oscillations are damped for a long time run. If the number of links is greater than 500, the disease spreads to the whole medium and then collapses. The relationship between normalized first coverage time and different probabilities is given by a power law as shown in the Fig. 5.11.

\subsubsection{Dynamic networks}

In the real world, people do not always travel to the same places, as in our study of static links, but to a variety of places. We therefore introduce "dynamic networks," where the long-distance interactions between cells change dynamically with time. The rule for the dynamic links is the following: each infected cell has a jump probability $p$ to contact with a cell chosen at random in the network.

After the circular wave was initiated in the center of the system, the state of each cell was detected each iteration. For each of the infected cells, whether it will contact non-locally with another cell depends on the jump probability $p$, which determines the total number of long-distance contacts. For example, if the jump probability is 0.1 , which means that each infected cell has a $10 \%$ possibility to jump, then at least one long-distance contact will occur if there are 10 infected cells. If the jumping probability is 1 , then all of the infected cells will have the chance to jump (contact) to other cells. The cell in which the long-distance contact occurs is chosen randomly in the network. To realize the non-diffusive contact, the destination cell was maintained with a light intensity of zero for a specific period of time. The destination cell could be an infected cell, in which case no new wave will be initiated. In addition, if the destination cell is located in the wave back of a wave, no new wave will be initiated, since the refractory state of the excitable medium is analogue to the recovery state 


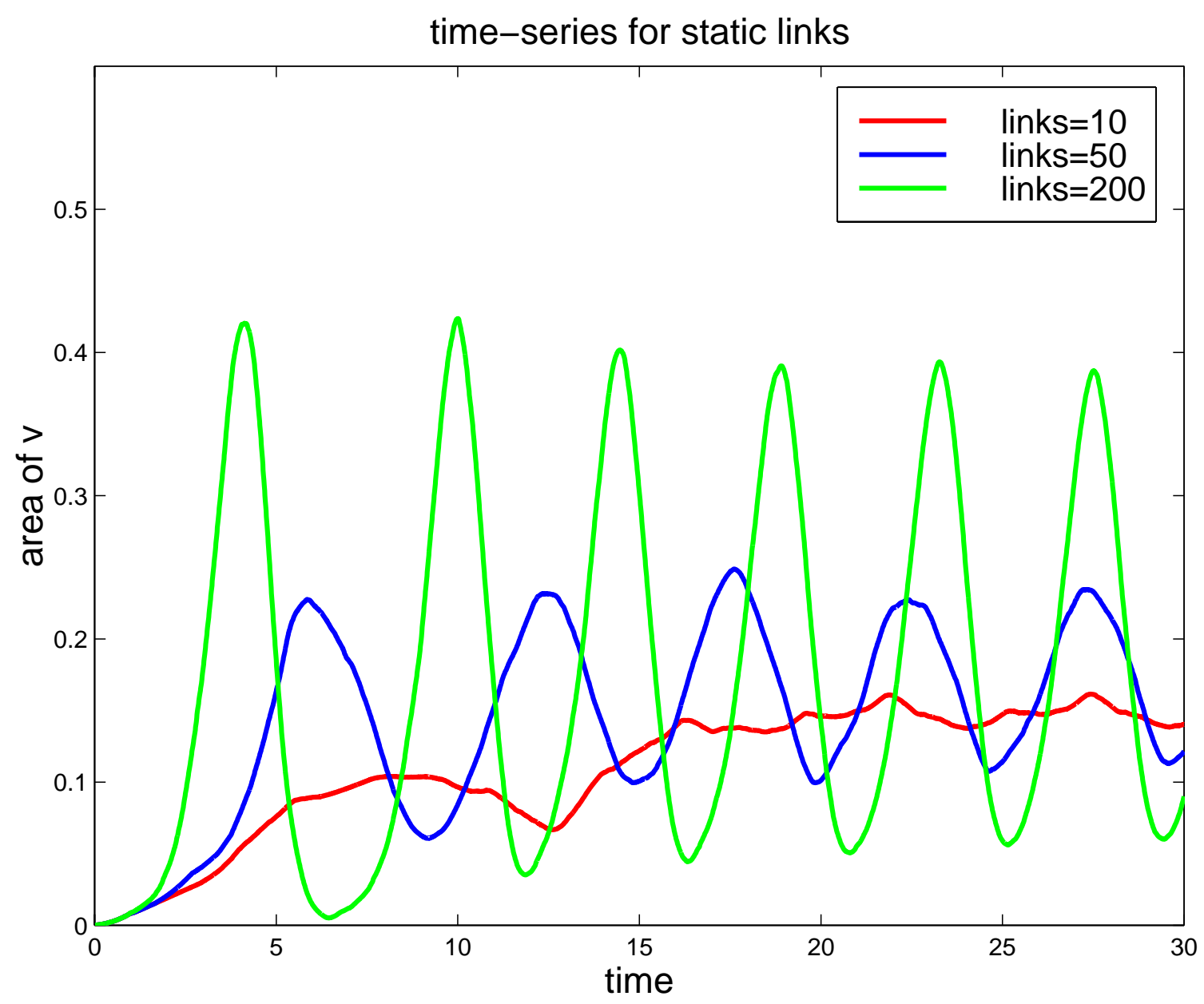

Figure 5.10. Numerical simulations of time-series of fraction of infected elements. Area $v$, which represents the fraction of the area of the oxidized catalyst, $\mathrm{Ru}(\mathrm{bpy})_{3}^{3+}$, in the medium, represents the fraction of infected elements. The number of static links: 10 (red), 50 (blue), and 200 (green). Conditions and other parameters of calculation are given in the text. 


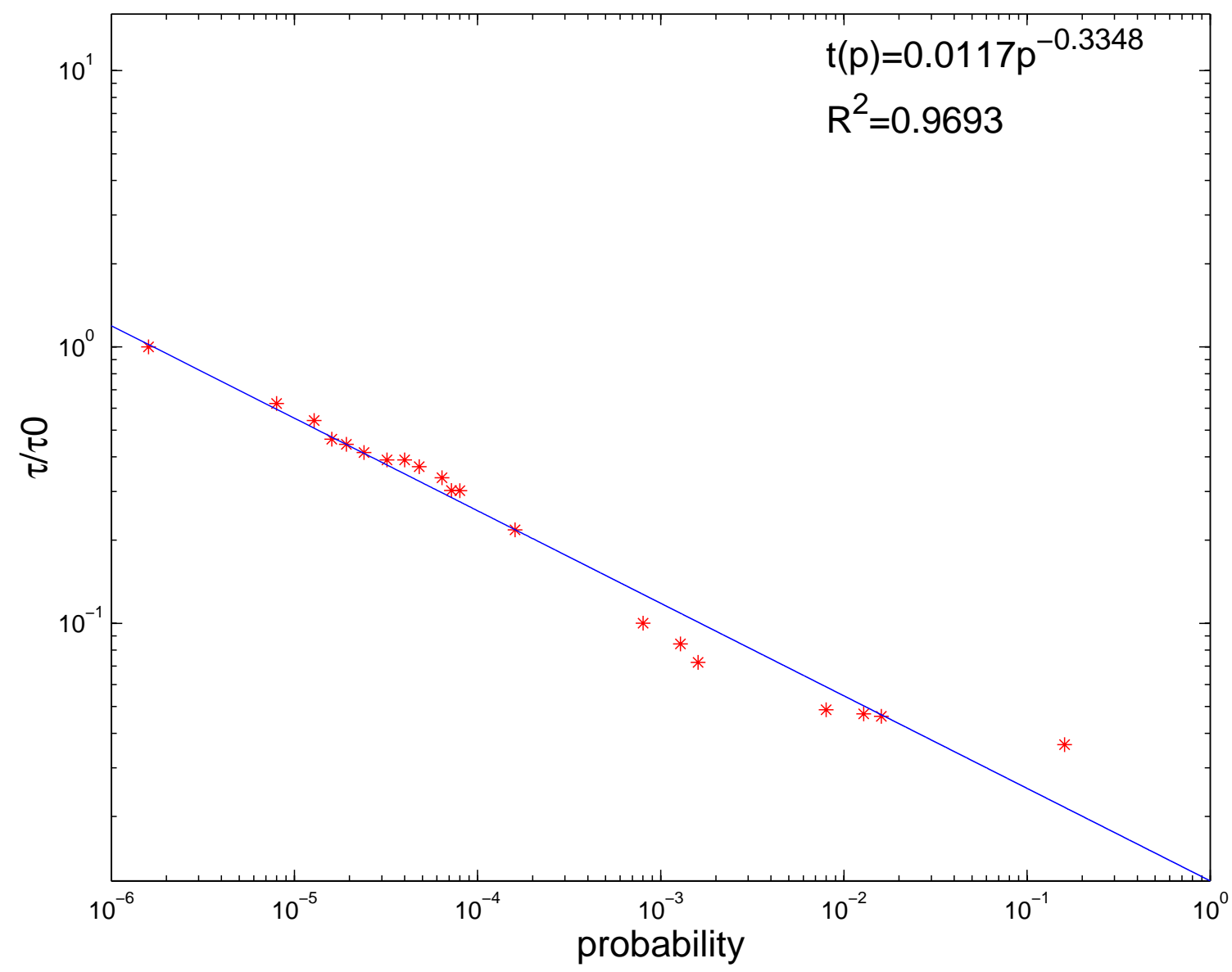

Figure 5.11. Simulation results of normalized first coverage time, $\tau / \tau(0)$, as a function of probability, $p . p$ is the ratio of static links over total possible links. $\tau$ is the time for each cell to be infected at least once. $\tau(0)$ is the time of one circular wave propagating from the center to cover the entire medium with $p$ set to 0 . The solid blue line is given by the power law $\tau / \tau(0)=0.0117 p^{-0.3348}$. Conditions and other parameters of calculation as in Fig. 5.10. 
of a disease. The time period for monitoring the system and imposing non-diffusive jumps was $6.0 \mathrm{~s}$. The time period for initiating non-diffusive jumps was $1.0 \mathrm{~min}$.

Examples of the spreading of an epidemic in a spatially extended population, represented by chemical waves propagating in the photosensitive BZ system with random non-local connections added, are illustrated in Figs. $5.12(p=0.1)$ and 5.13 $(p=0.15)$. The spatiotemporal behavior of outbreaks of the epidemic, represented by the fraction of excited elements, changed from irregular to periodic when $p$ was increased, as shown in Figs. of 5.14(a) - 5.16(a). The changing from irregular to periodic was demonstrated by the corresponding power spectrum, as shown in Figs. 5.14(a) - 5.16(a). The periodic outbreak reveals the global synchronization of the outbreak of the epidemic in the entire medium. All of the jumps were randomly selected such that the new waves generate at a random place; however, the overall average behavior of the system is synchronized, which implies a phase transition from a regime without synchronization to one with synchronization. The first coverage times for various values of $p$ were recorded and normalized by the first coverage time for $p=0$. A power law is obtained for the normalized coverage time vs $p$ in a log-log plot, as shown in Fig. 5.17.

Figure 5.18 illustrates numerical simulations of disease spreading in a spatially extended population with non-diffusive connections $(p=0.0004)$. The spatiotemporal dynamics of the disease spreading is shown in the Figs. 5.19(a) and 5.20(a). These figures illustrate the changing of outbreaks of the disease from irregular to periodic when the jump probability increases, and Figs. 5.19(b) and 5.20(b) show the power spectra of the corresponding time series. When the probability is greater than 0.000425, the system collapses, which means that the disease is pervasive throughout the medium and the populations recovers simultaneously. A power law is obtained for the normalized first coverage time vs. $p$ in the numerical simulations, as shown in Fig. 5.21.

The above study was carried out in a homogeneous medium, in which each element 


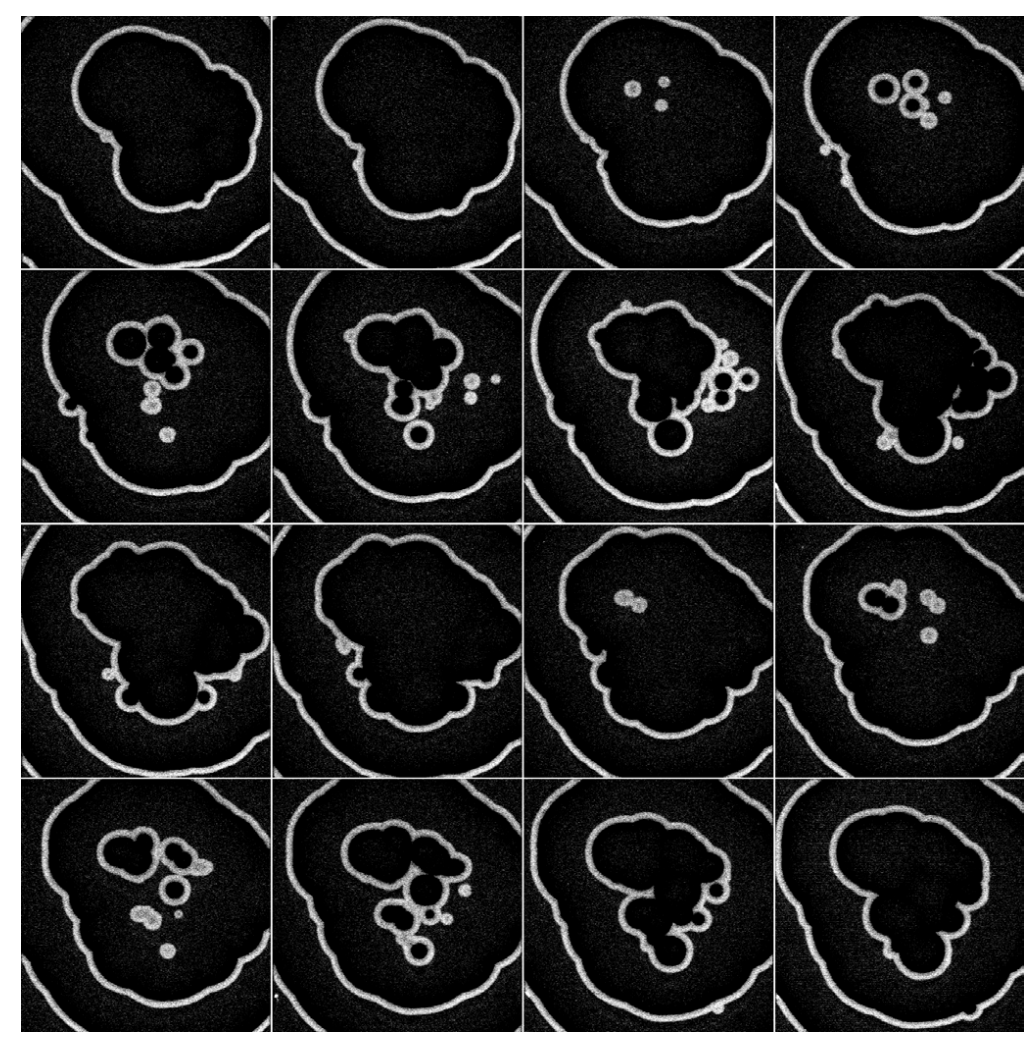

Figure 5.12. Images showing the time sequences of chemical wave propagation with random non-diffusive connections in the excitable BZ medium (time increases from left to right and then to the next row). The time interval between each frame is $6.0 \mathrm{~s}$. The gray level in each panel is proportional to the concentration of the catalyst, $\mathrm{Ru}(\mathrm{bpy})_{3}^{3+}$, with white and black representing high and low values, respectively. Every infected cell jumps to a random position in the medium with the probability of 0.1 . Other experimental conditions are given in the text. 


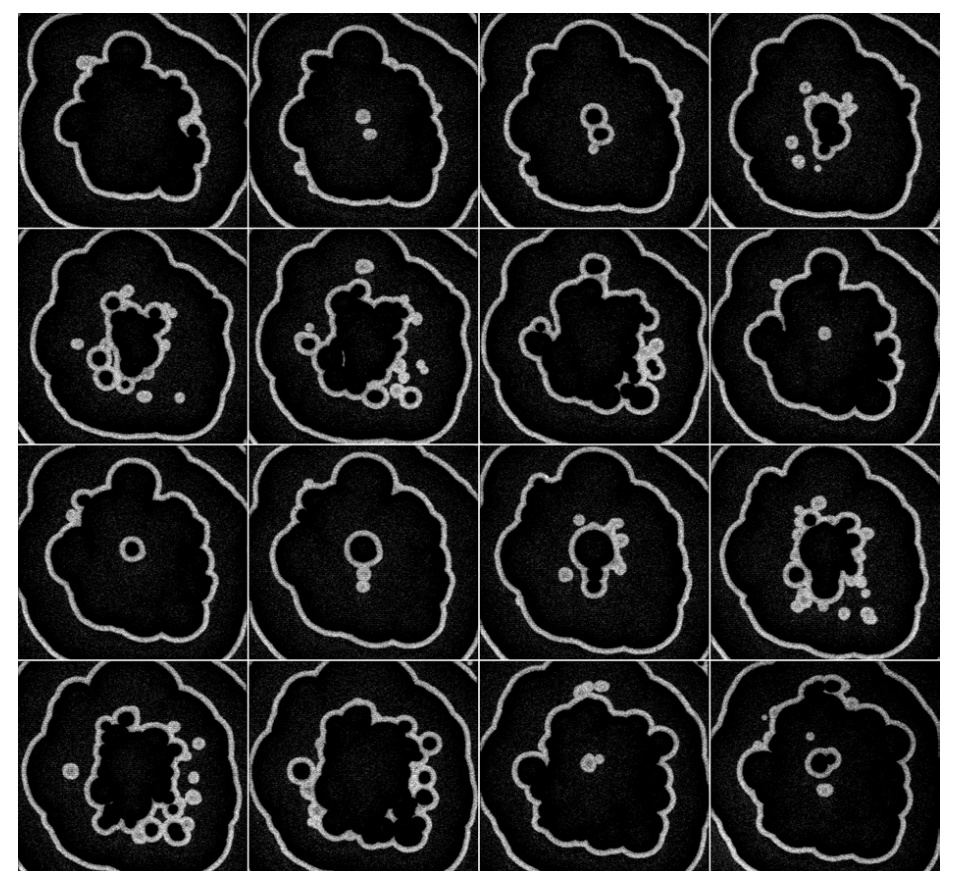

Figure 5.13. Images showing the time sequences of chemical wave propagation with random non-diffusive connections in the excitable BZ medium (time increases from left to right and then to the next row). The jump probability is 0.15 . Other conditions as in Fig. 5.12. 


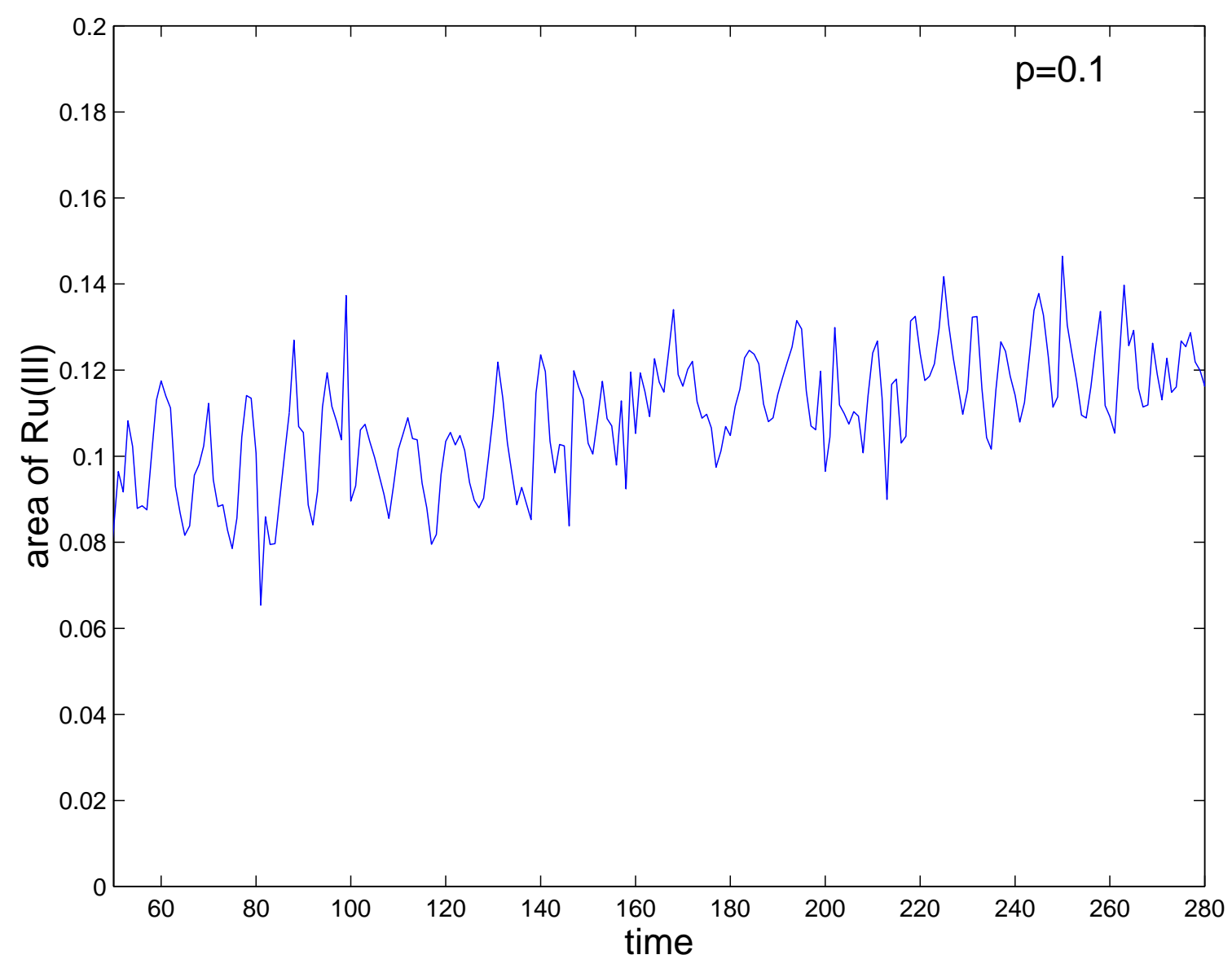

Figure 5.14(a). Time-series of fraction of infected elements in the experiment. Area of $\mathrm{Ru}(\mathrm{III})$, which represents the fraction of the area of the oxidized catalyst, $\mathrm{Ru}(\mathrm{bpy})_{3}^{3+}$, in the medium, represents the fraction of infected elements. Time is represented by the frame number. The probability of jumping for each infected cell is 0.10 . Other conditions as in Fig. 5.12. 


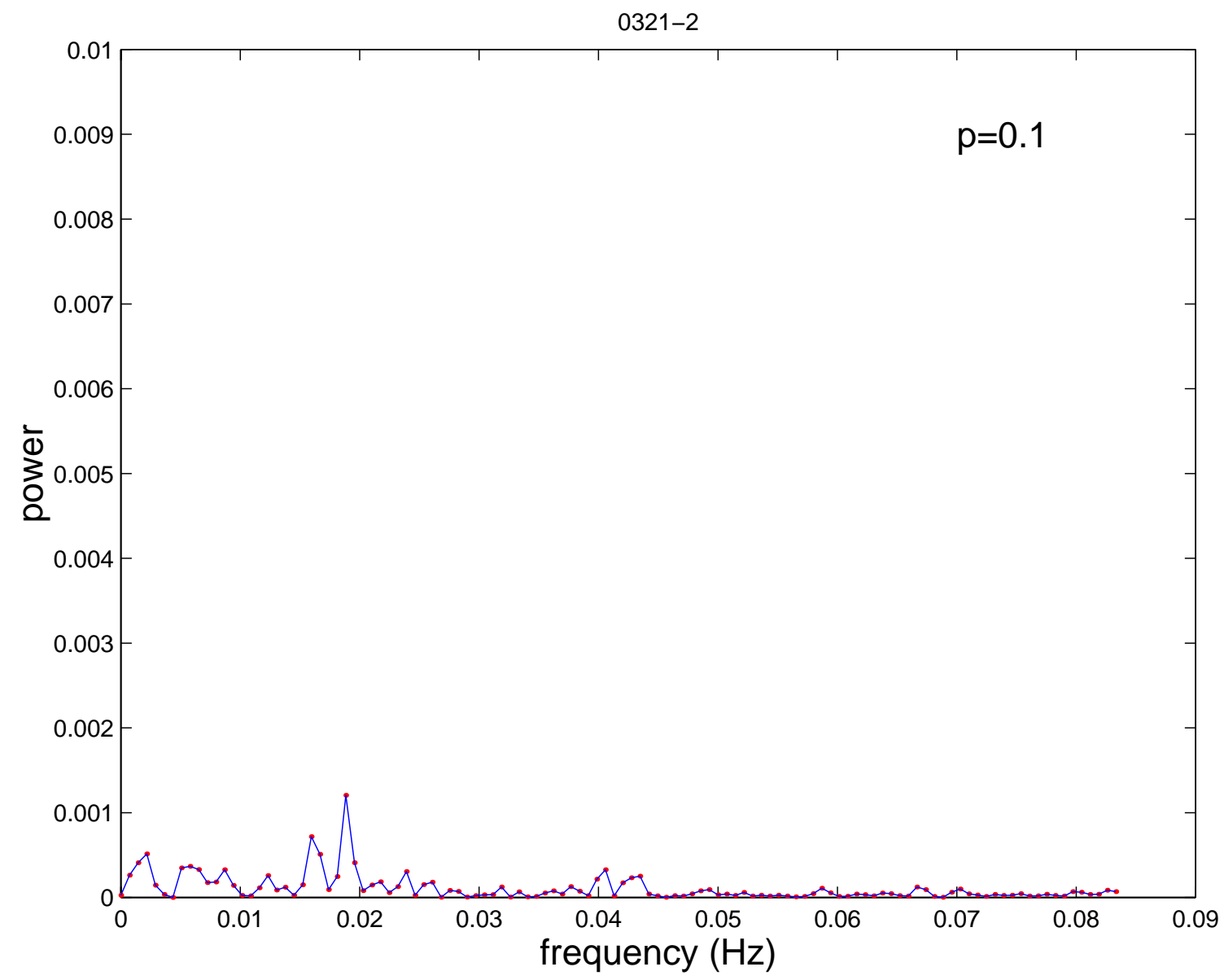

Figure 5.14(b). Power spectrum of the time-series of fraction of infected elements in the experiment for $p=0.10$. 


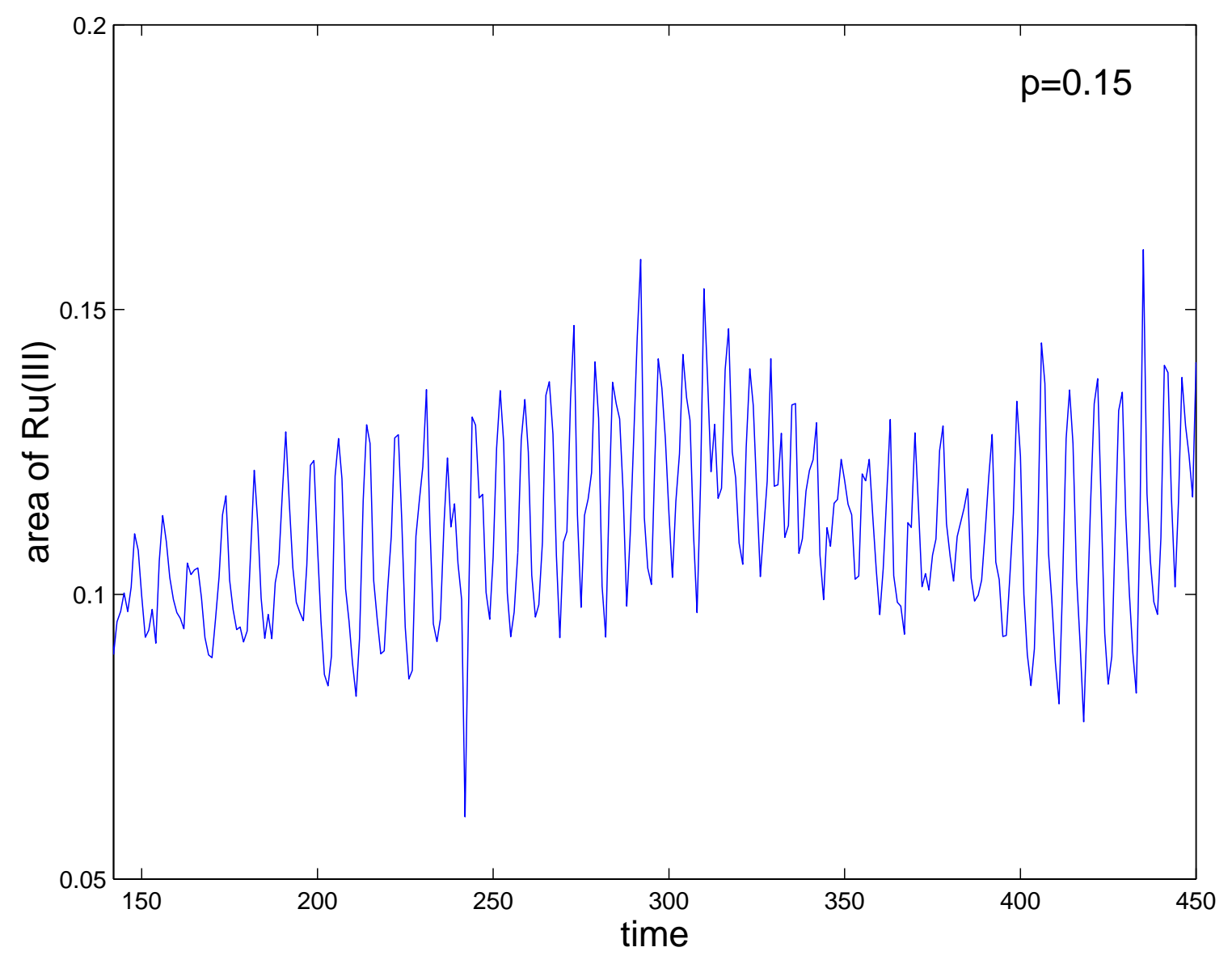

Figure 5.15(a). Time-series of fraction of infected elements in the experiment for $p=$ 0.15. Area of $\mathrm{Ru}(\mathrm{III})$, which represents the fraction of the area of the oxidized catalyst, $\mathrm{Ru}(\mathrm{bpy})_{3}^{3+}$, in the medium, represents the fraction of infected elements . Time is represented by the frame number. Experimental conditions as in Fig. 5.12. The wave evolution in frames 254-269 is shown in Fig. 5.13. 


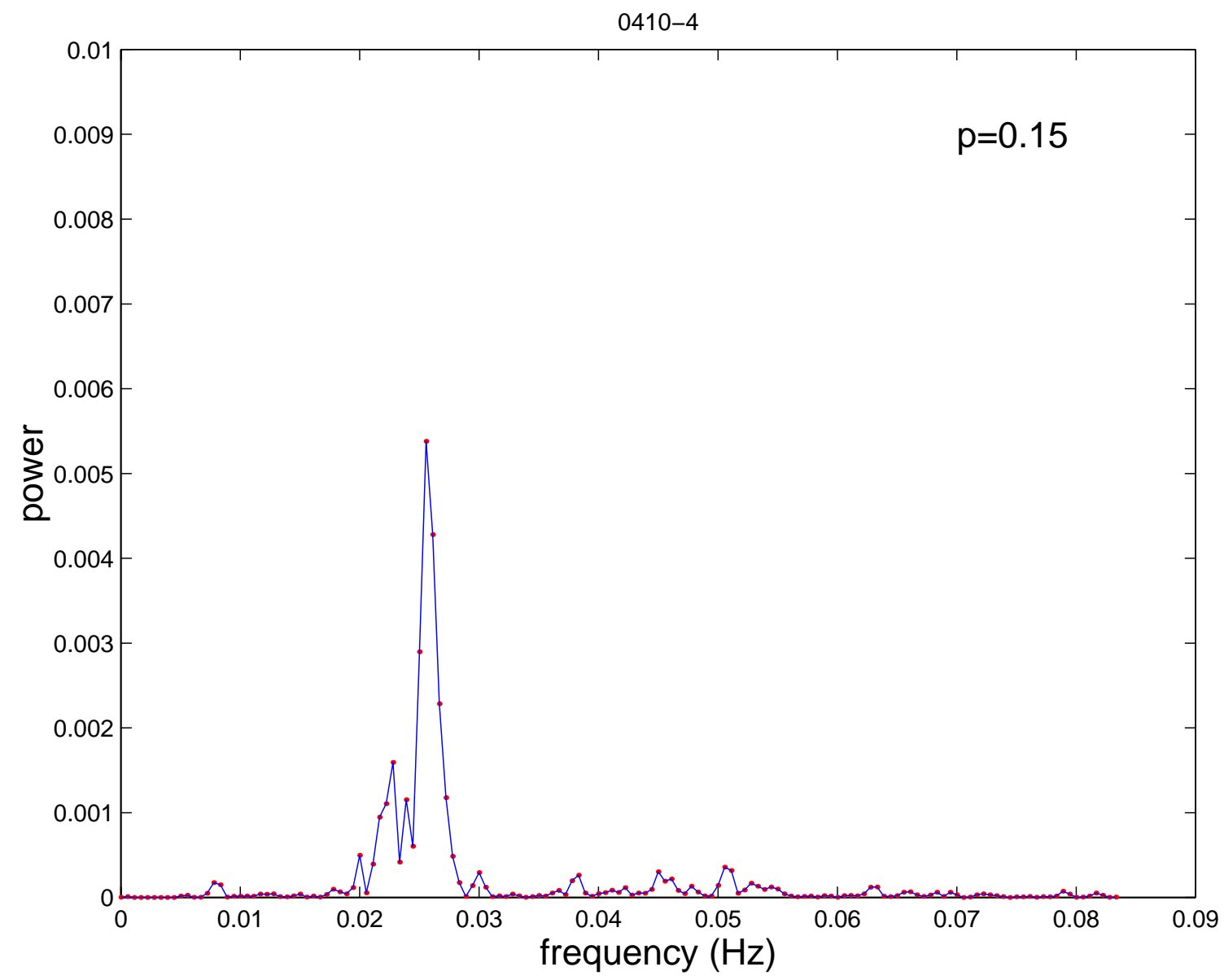

Figure 5.15(b). Power spectrum of the time-series of fraction of infected elements in the experiment for $p=0.15$. 


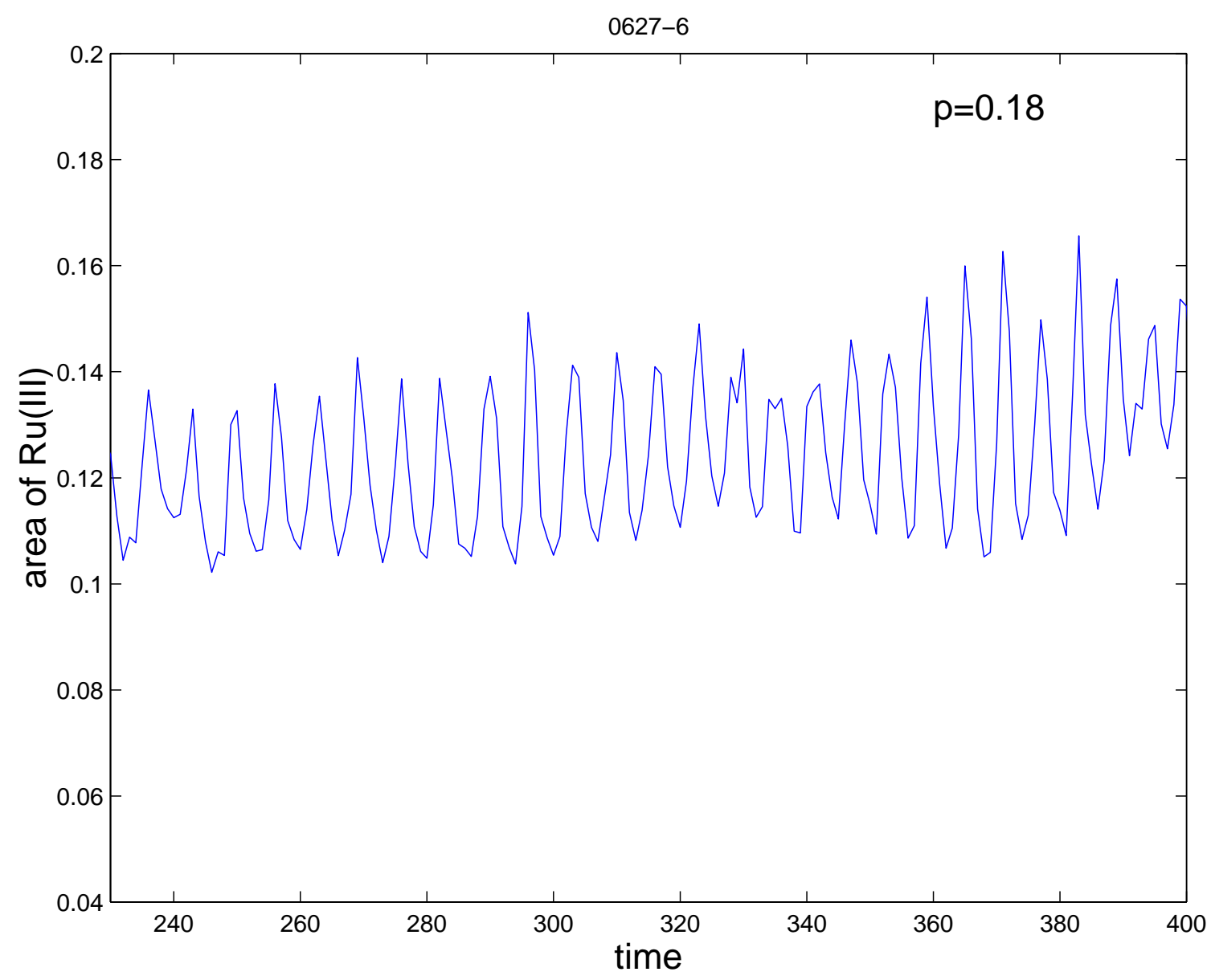

Figure 5.16(a). Time-series of fraction of infected elements in the experiment. Area of $\mathrm{Ru}(\mathrm{III})$, which represents the fraction of the area of the oxidized catalyst, $\mathrm{Ru}(\mathrm{bpy})_{3}^{3+}$, in the medium, represents the fraction of infected elements. Time is represented by the frame number. The probability of jump for each infected cell is 0.18 . Other conditions as in Fig. 5.12 . 


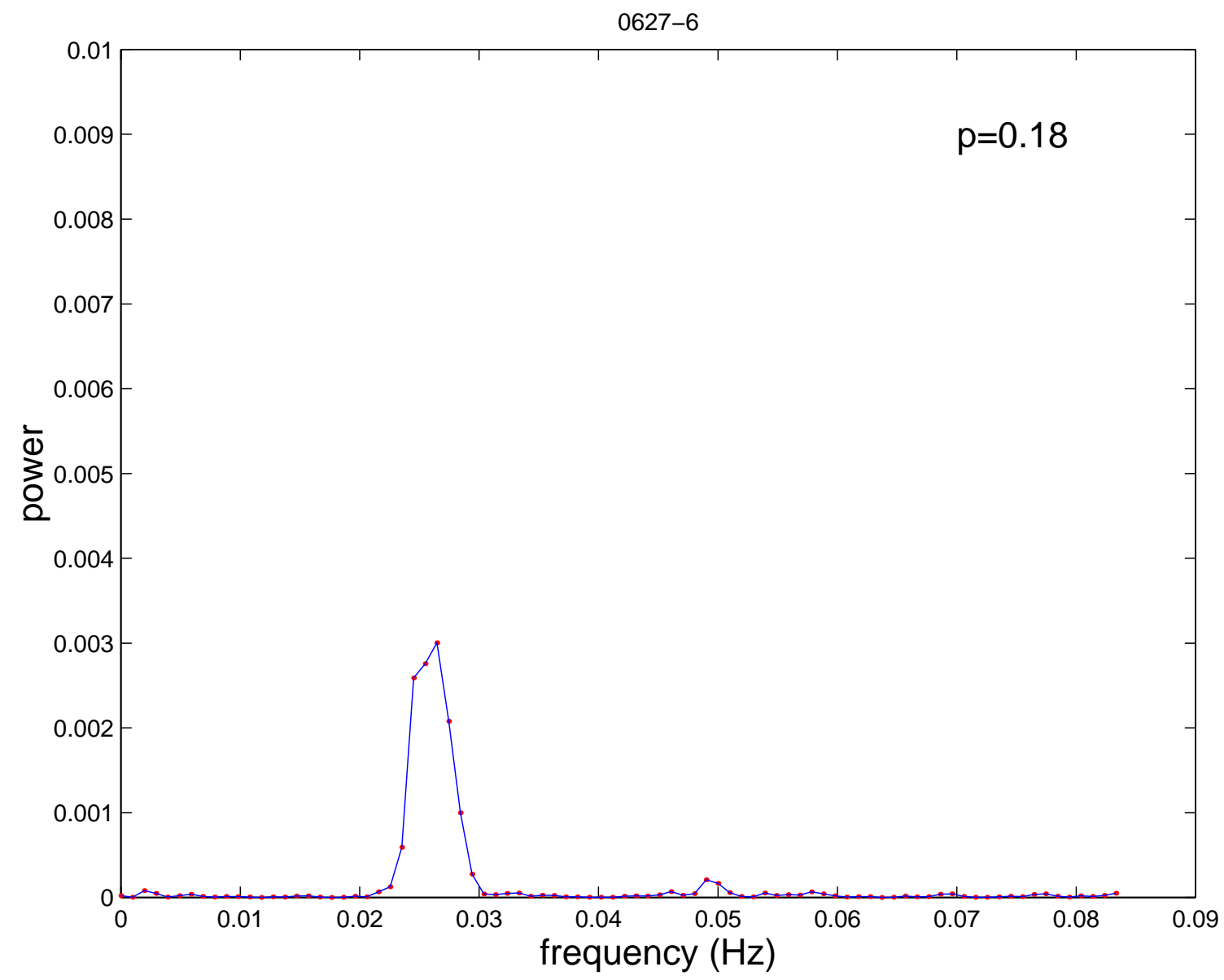

Figure 5.16(b). Power spectrum of the time-series of fraction of infected elements in the experiment for $p=0.18$. 


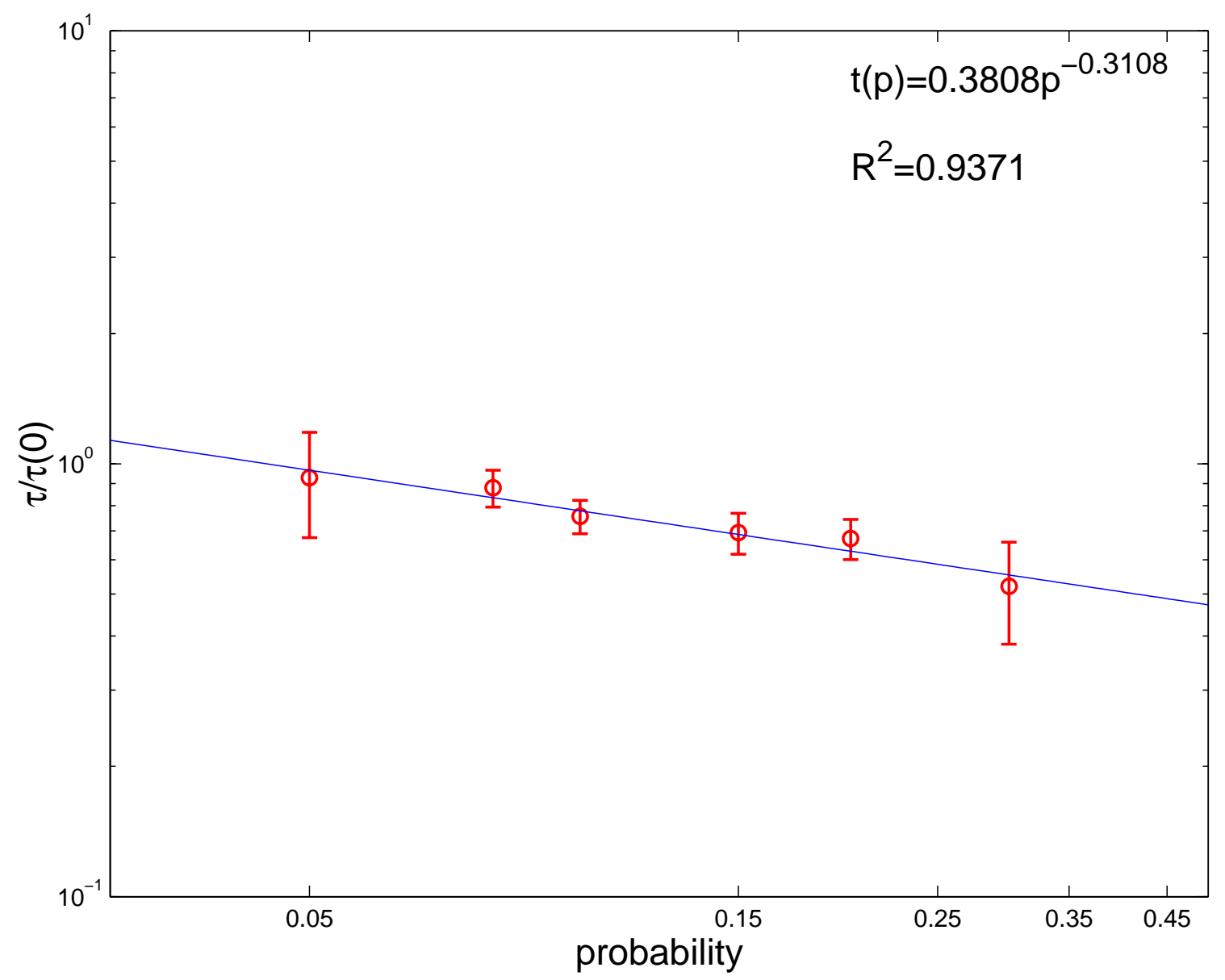

Figure 5.17. Experimental results of normalized coverage time, $\tau / \tau(0)$, as a function of probability, $p . p$ is the probability of randomly jumping for each infected cell. $\tau$ is the time for infecting each cell at least once. $\tau(0)$ is the time of one circular wave propagating from the center to cover the entire medium for $p$ set to 0 . The solid blue line is given by the power law $\tau / \tau(0)=0.3803 p^{-0.3108}$. The red circles show mean values from $5-6$ measurements and bars indicate standard deviations. 


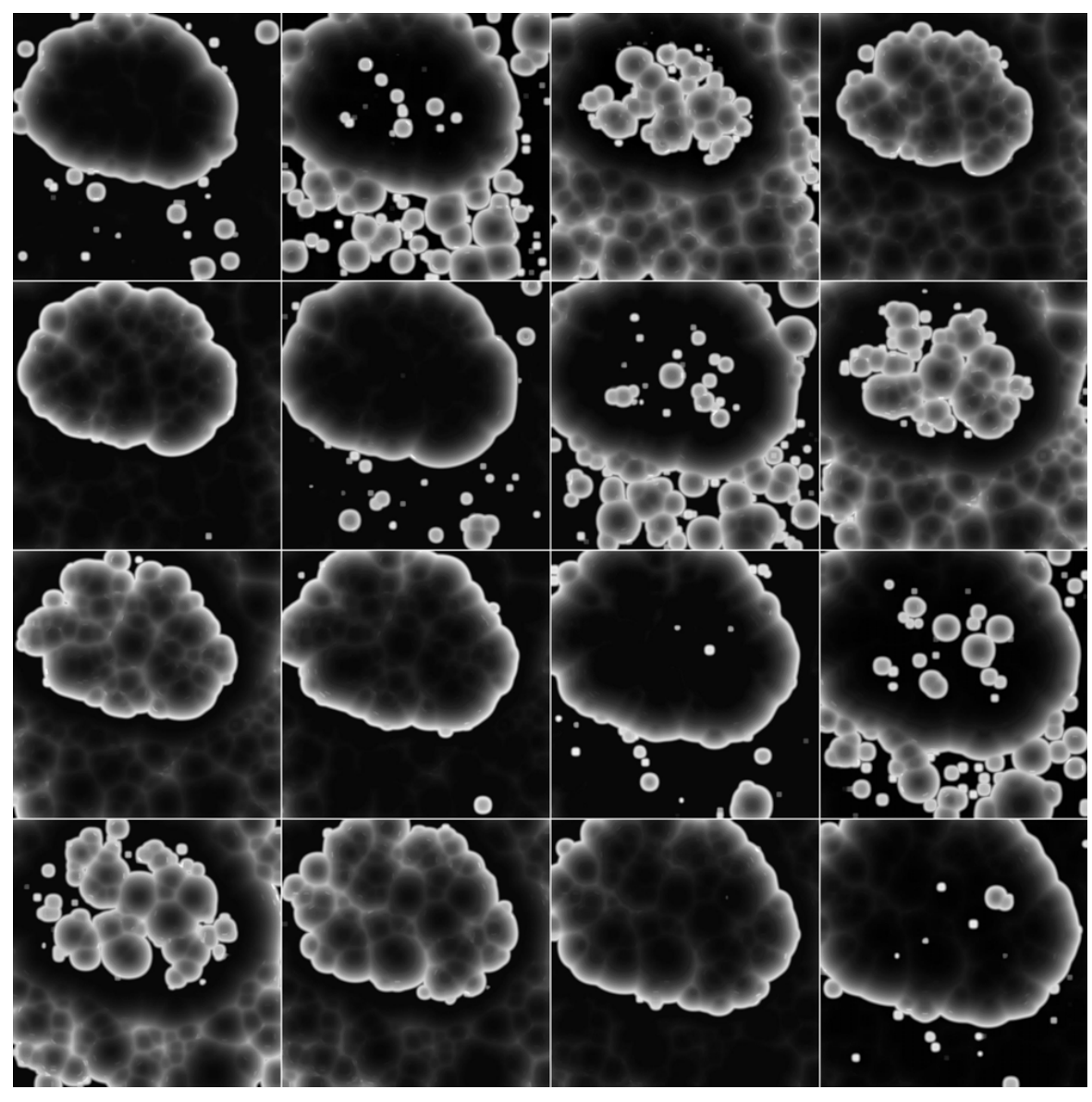

Figure 5.18. Images showing the time sequences of chemical wave propagation with random non-diffusive connections from the numerical integration of the modified Organetor. The time interval between each frame is 500 time steps. The gray level [given by $\left.\operatorname{int}\left(v / v_{\max } \times 255\right)\right]$ represents the concentration of the catalyst, $\mathrm{Ru}(\mathrm{bpy})_{3}^{3+}$, with white and black representing high and low values, respectively. Infected cells jump to a random position in the medium with the probability of 0.0004 . Other conditions are described in the text. 


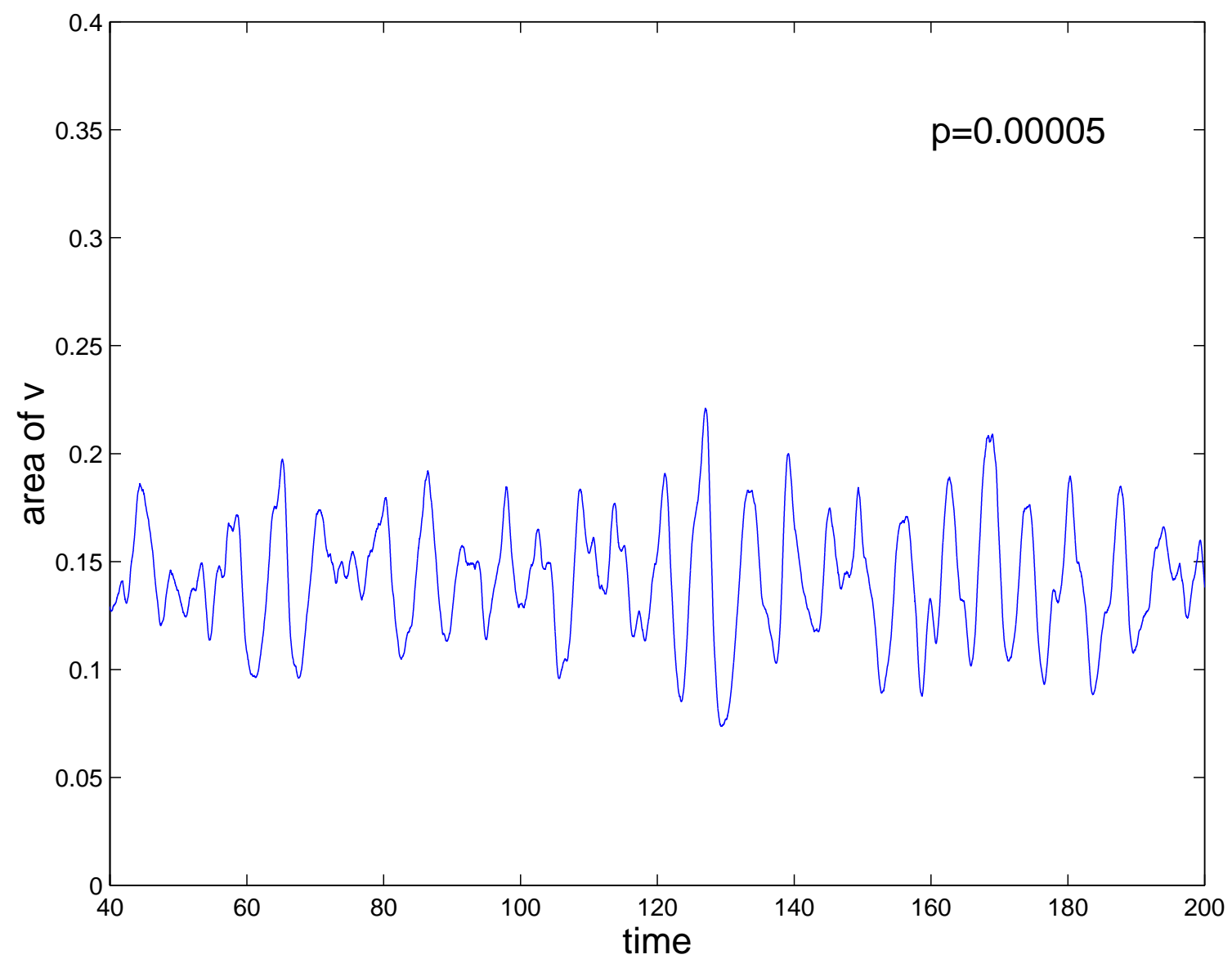

Figure 5.19(a). Numerical simulations of time-series of fraction of infected elements. Area of $v$, which represents the fraction of the area of the oxidized catalyst, $\mathrm{Ru}(\mathrm{bpy})_{3}^{3+}$, in the medium, represents the fraction of infected elements. The probability of jumping for each infected cell is 0.00005. Conditions and other parameters of calculation as in Fig. 5.18. 


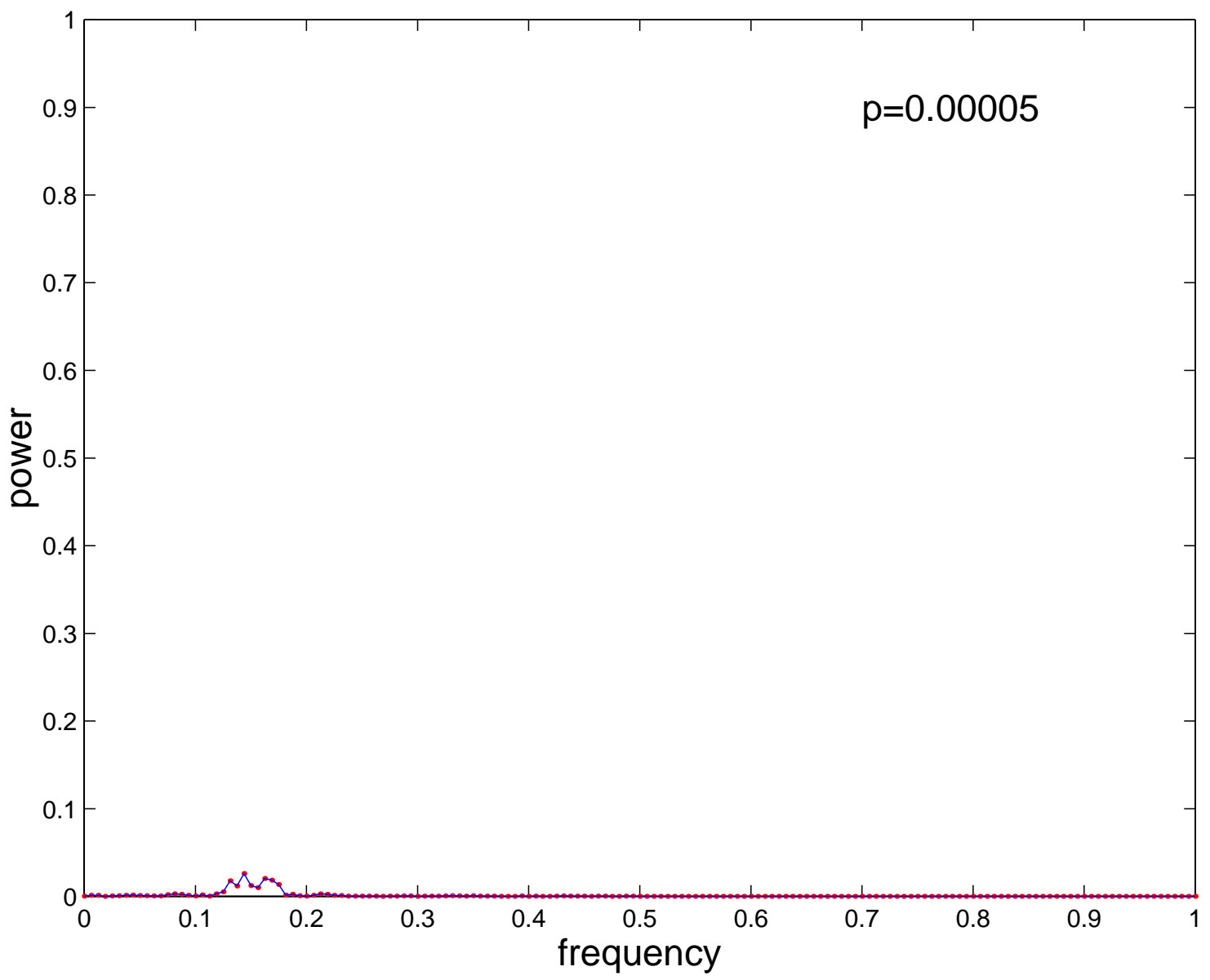

Figure 5.19(b). Power spectrum of time-series for $p=0.00005$. Conditions and other parameters of calculation as in Fig. 5.18. 


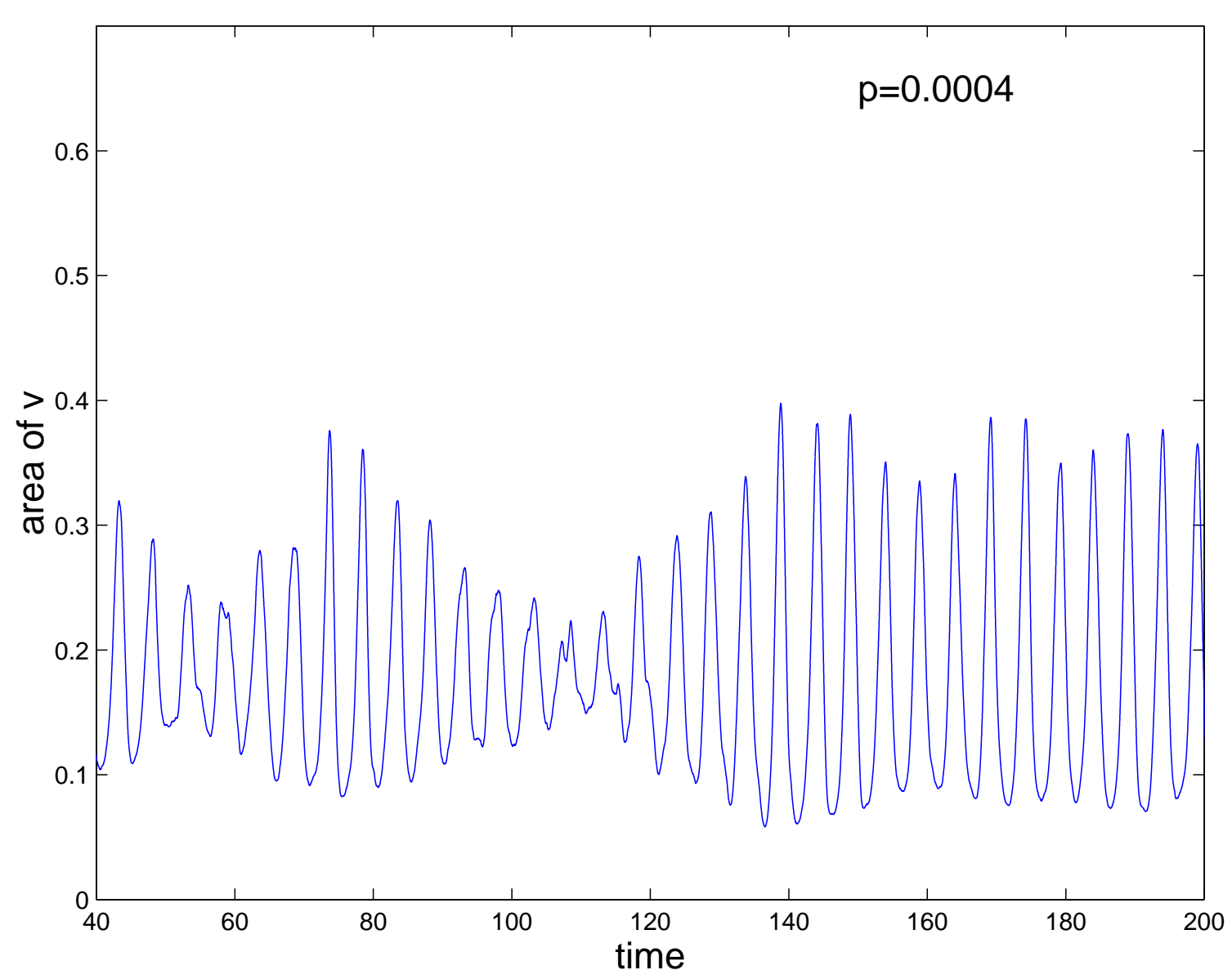

Figure 5.20(a). Numerical simulations of time-series of fraction of infected elements. Area $v$, which represents the fraction of the area of the oxidized catalyst, $\mathrm{Ru}(\mathrm{bpy})_{3}^{3+}$, in the whole medium, represents the fraction of infected elements. The probability of jumping for each infected cell is 0.0004. Conditions and other parameters of calculation as in Fig. 5.18. The wave evolution for 150-175 is shown in Fig. 5.18. 


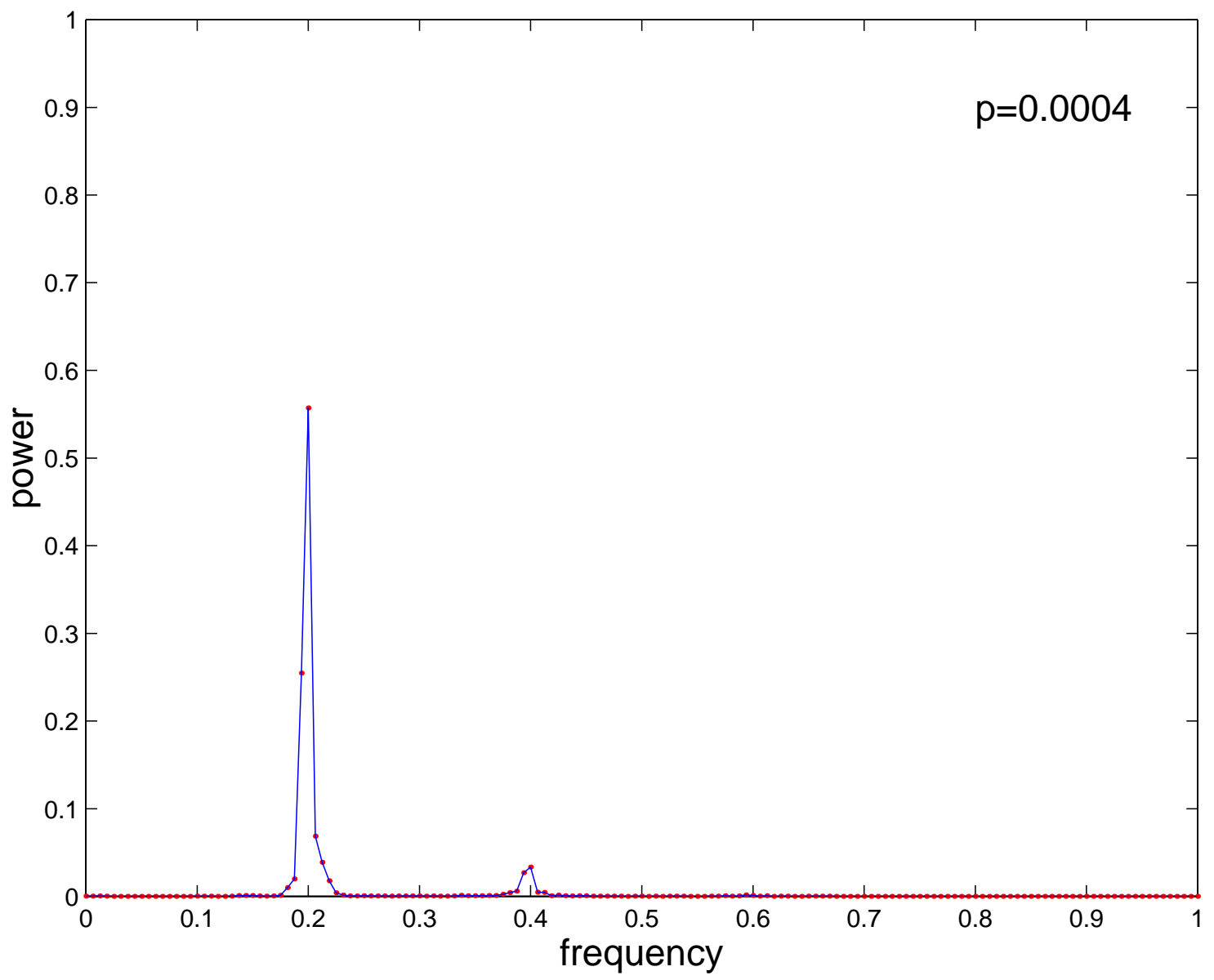

Figure 5.20(b). Power spectrum of time-series for $p=0.0004$. Conditions and other parameters of calculation as in Fig. 5.18. 


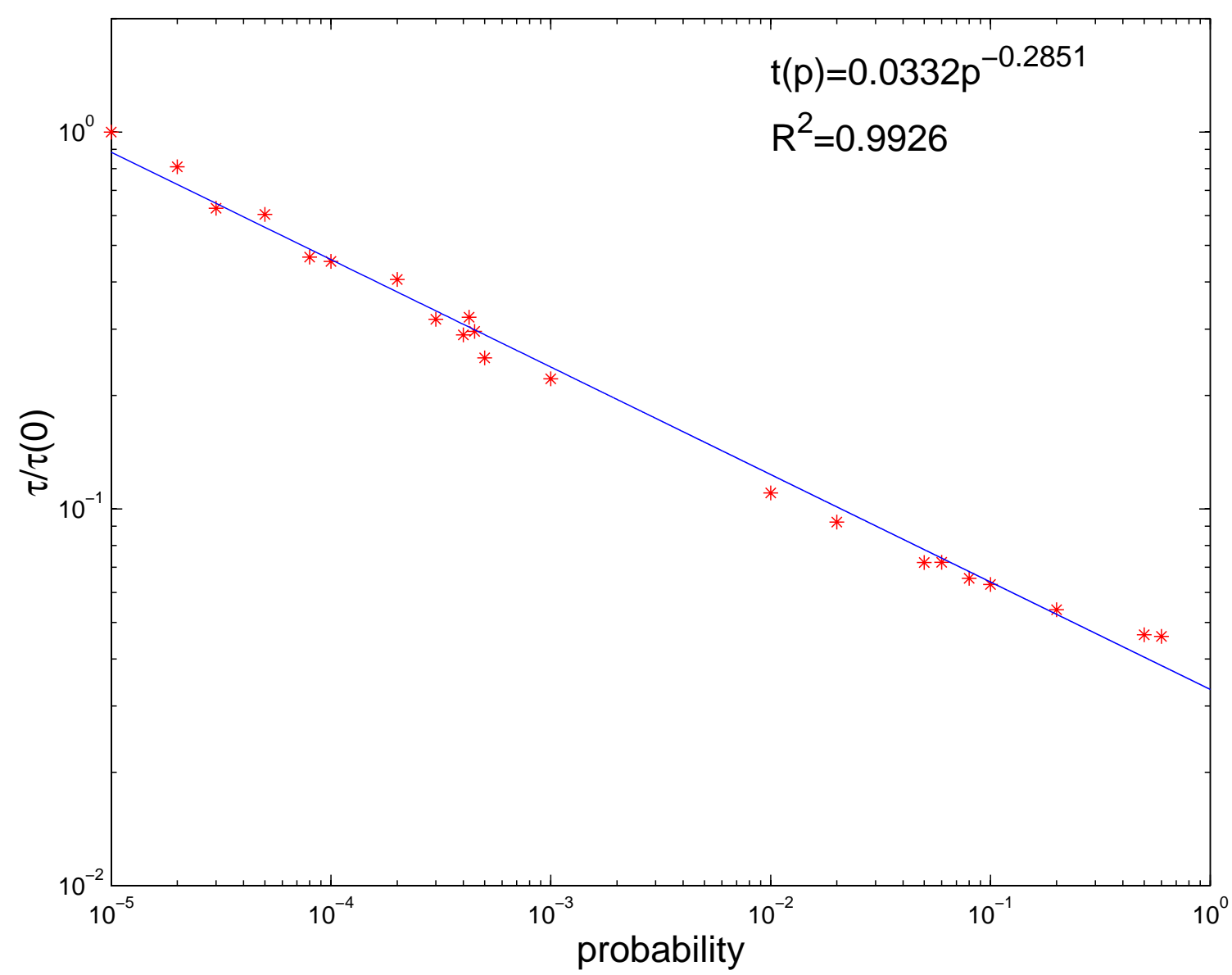

Figure 5.21. Simulation results of normalized coverage time, $\tau / \tau(0)$, as a function of probability, $p$. $p$ is the probability of random jumps for each infected cell. $\tau$ is the time for each cell to be infected at least once. $\tau(0)$ is the time of one circular wave propagating from the center to cover the entire medium with $p$ set to 0 . The solid blue line is given by the power law $\tau / \tau(0)=0.0332 p^{-0.2851}$. Conditions and other parameters of calculation as in Fig. 5.18. 
has the same excitability and is initially equally susceptible to the disease. We also studied inhomogeneous media in numerical simulations. The heterogeneity was realized by adjusting the light intensity $\phi$ in each cell according to $\phi=\phi_{0}\left[1+\frac{1}{2} G(x, \sigma)\right]$ with $|x| \leq 2 \sigma$, where $\phi_{0}$ is the reference intensity of 0.073 , and $G(x, \sigma)$ is a Gaussian distribution random number with a standard deviation of $\sigma$. The heterogeneity is controlled by $\sigma$. The same inhomogeneity was maintained throughout the simulation. When the heterogeneity was low $(0.1<\sigma<0.5)$, the behavior of the disease spreading was not affected. This was also found in bond percolation modeling of disease spreading [47], where a weak heterogeneity does not affect the behavior. However, when the heterogeneity is sufficiently high $(\sigma>0.6)$, chemical waves will be initiated at the low excitability regions, and the disease spreading in the network is not determined by the long-distance jumps but, rather, by the inhomogeneity itself. After a short time, the coverage of the excited elements is the same for different jump probabilities when $\sigma=0.9$. It is understandable if we consider the case of noise driven avalanche behavior in Ref. [74], where the chemical wave was induced by spatiotemporal noise. In our case, the noise was fixed and there is no doubt that the chemical wave will be generated by the heterogeneity itself.

\subsubsection{Domain model networks}

In this part of our study, we construct different domains that are separated from each other. The long-distance interactions occur between cells belonging to different domains. Within one domain, only local connections by diffusion can occur. Put

in the other way, the disease is always initiated by an infected element coming from outside the domain; it then spreads in the domain by local contacts or to other domains by long-distance contacts.

The relationship between the number of cells in domains (the population of cities) and the rank of the domains follows Zipf's law [75,76], which is named after the 
linguistic professor George Kinsley Zipf. In our network, there are 40 domains, whose populations and the number of domains are listed in Table 5.1. The line in Fig. 5.22 shows Zipf's law of the ranks of domains and their populations, both of which are in natural logarithm scale. The slope is close to -1 , which is the value found for the size of the large cities in the USA, India, and France [76].

Table 5.1. Population distribution in the domains

\begin{tabular}{|l|l||l|l||l|l|}
\hline $\begin{array}{l}\text { number } \\
\text { of domain }\end{array}$ & $\begin{array}{l}\text { number of cells } \\
\text { in domain }\end{array}$ & $\begin{array}{l}\text { population } \\
\text { of domain }\end{array}$ & $\begin{array}{l}\text { rank } \\
\text { of domain }\end{array}$ & $\ln$ (rank) & $\ln$ (size) \\
\hline 1 & 260 & 26000 & 1 & 0 & 10.1658 \\
2 & 190 & 19000 & 2 & 0.6931 & 9.8522 \\
7 & 100 & 1000 & 3 & 1.0986 & 9.2103 \\
30 & 50 & 5000 & 4 & 1.3863 & 8.5172 \\
\hline
\end{tabular}

The construction of the domains in the network is taken in the order of decreasing size. The domain with the largest population is set at the center of the network. All the centers of the other domains are located randomly and the domains do not overlap with each other. The gaps between cities, where the disease cannot spread, are maintained as non-excitable by high intensity of light. Figure 5.23 shows a particular distribution of the domains in our network with $1000 \times 1000$ elements. The darker areas represent domains and each dot represents a cell with $10 \times 10$ elements. The white areas represent the gaps between the domains. This network was used in the numerical simulations. As in the dynamic networks, each infected cell has a fixed probability to jump to another cell, which cannot be in the same domain.

The first wave was initiated at the center of the largest domain. Each infected cell has a fixed probability $p$ to jump to another cell chosen randomly in any of the other domains. In the simulations, the destination cell was maintained at zero light intensity for 100 time steps. The time period for detecting infected cells and updating the longdistance interaction cells was one time step. Examples of the spreading of an epidemic 


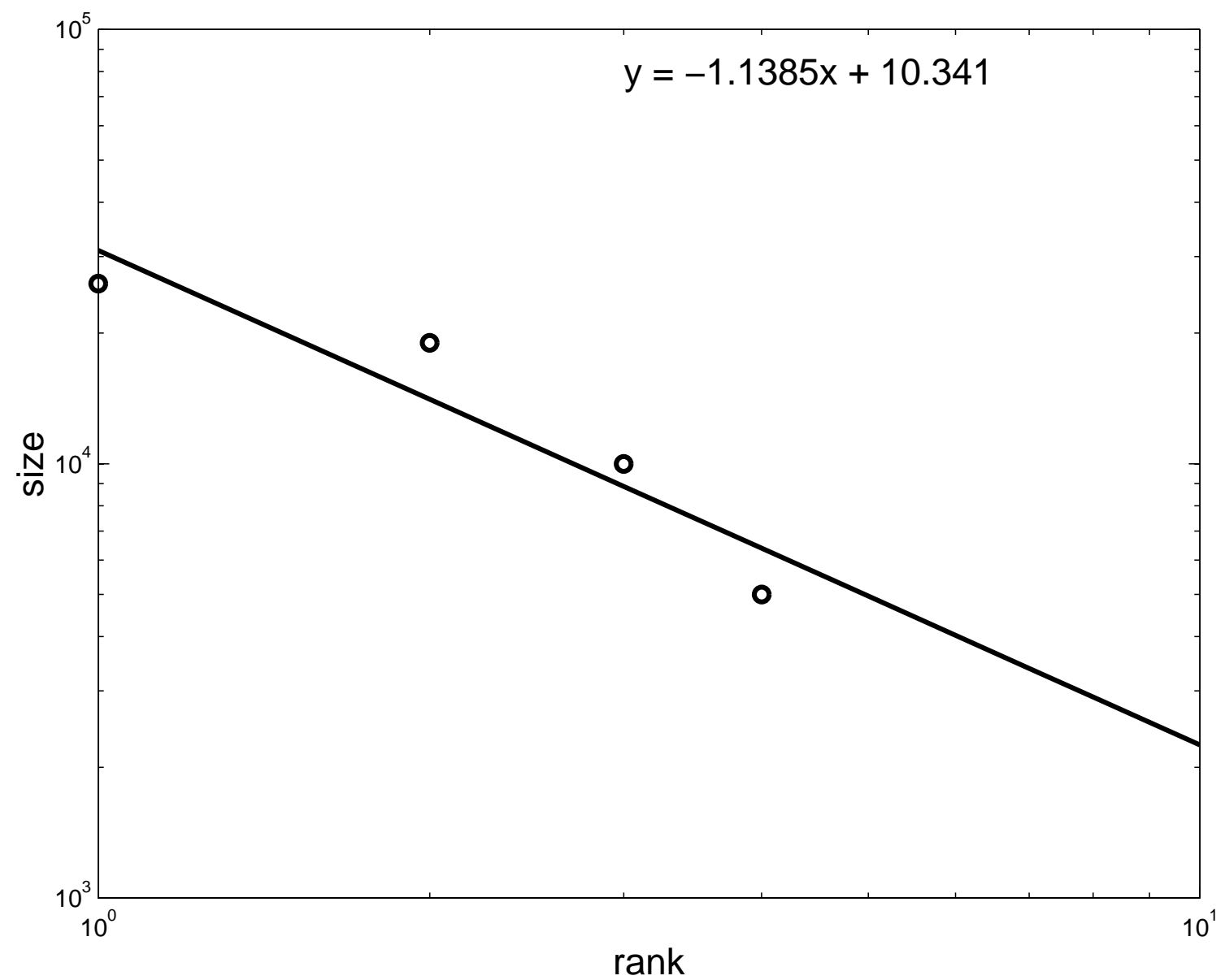

Figure 5.22. Zipf's law relationship between the size and the rank of the 4 different sizes of the domains. Size represents the population (the number of elements) of the domain and rank represents the order of the size, largest as 1 , then 2 and so on. 


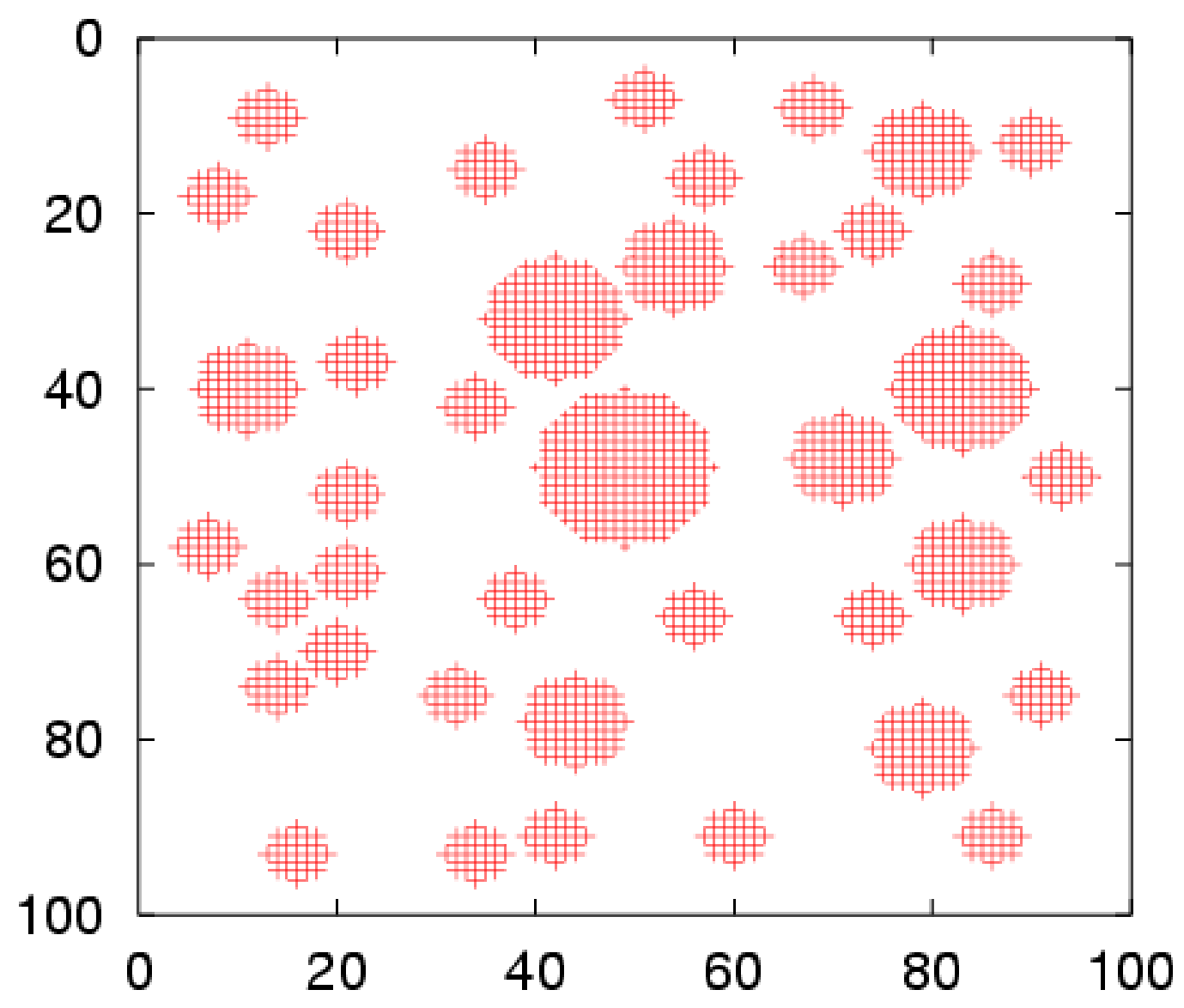

Figure 5.23. Domains distribution used in the numerical simulations of domain model. 

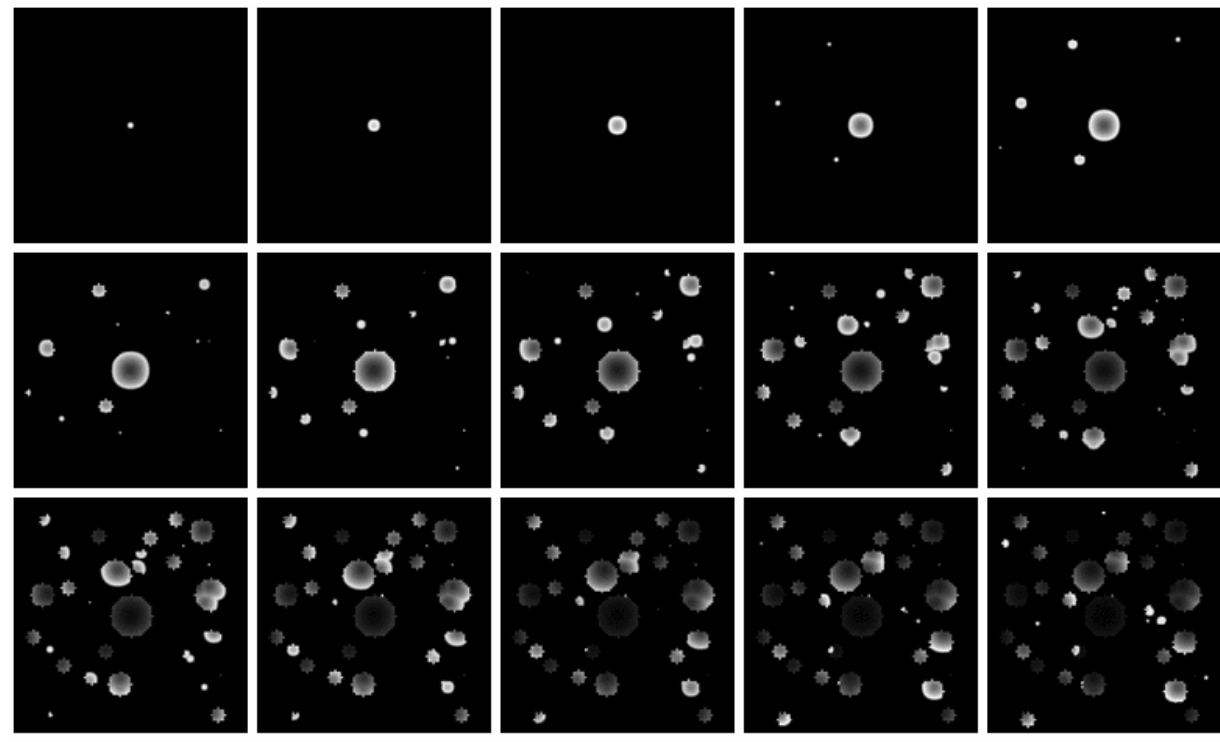

Figure 5.24. Images showing the time sequences of chemical wave propagation for with random non-diffusive connections in the domain model in Fig. 5.23 from the numerical integration of the modified Organetor. The time interval between each frame is 500 time steps. The gray level [given by $\operatorname{int}\left(v / v_{\max } \times 255\right)$ ] represents the concentration of the catalyst, $\mathrm{Ru}(\mathrm{bpy})_{3}^{3+}$, with white and black representing high and low values, respectively. Infected cells jump to randomly chosen cells in any other domain with the probability of 0.00008. Other conditions are described in the text. 
in a domain model with dynamic links are illustrated in Figs. $5.24(p=0.00008)$ and $5.25(p=0.0008)$. The time sequence of the fraction of excitation for different jump probabilities is shown in Fig. 5.26. The time series of the fraction of excitation is irregular but sustained for small probabilities, and the amplitude increased as the probability increased. However, the system collapses following a regular sustained oscillation of the coverage for $p=8.0 \times 10^{-4}$.

\subsubsection{Discussion}

We have investigated the spatiotemporal dynamics of disease spreading in three different complex networks, static, dynamic, and domain models. We use the excitable media of the photosensitive BZ system to mimic the SIRS model in experiments as well as in numerical simulations. In our networks, the local interactions are considered as well as the long-distance interactions. Our results have demonstrated the effects of the long-distance interactions on disease spreading. Variations in the numbers of static links or in the jump probability significantly influence the outcome of epidemic spreading. In addition, we find that with more long distance interactions in the network, the faster the disease spreading to the network.

Models of mobile individuals are interesting because they represent more realistic situations in social contacts. Infections spreading due to mobility is an important factor for the spreading of diseases, in contrast with models where diseases spread only between individuals. In the various models that take into account traveling [21-30], all of the individuals are allowed to move and the transmission occurs only if a susceptible individual contacts with an infected individual; otherwise, nothing happens. In our studies, we consider only travel of infected individuals, which is the most important travel compared to those of other individuals (susceptible and recovery) as claimed in [25]. We have considered both static and dynamic models, while in other models only non-random travel was considered [21-25]. 

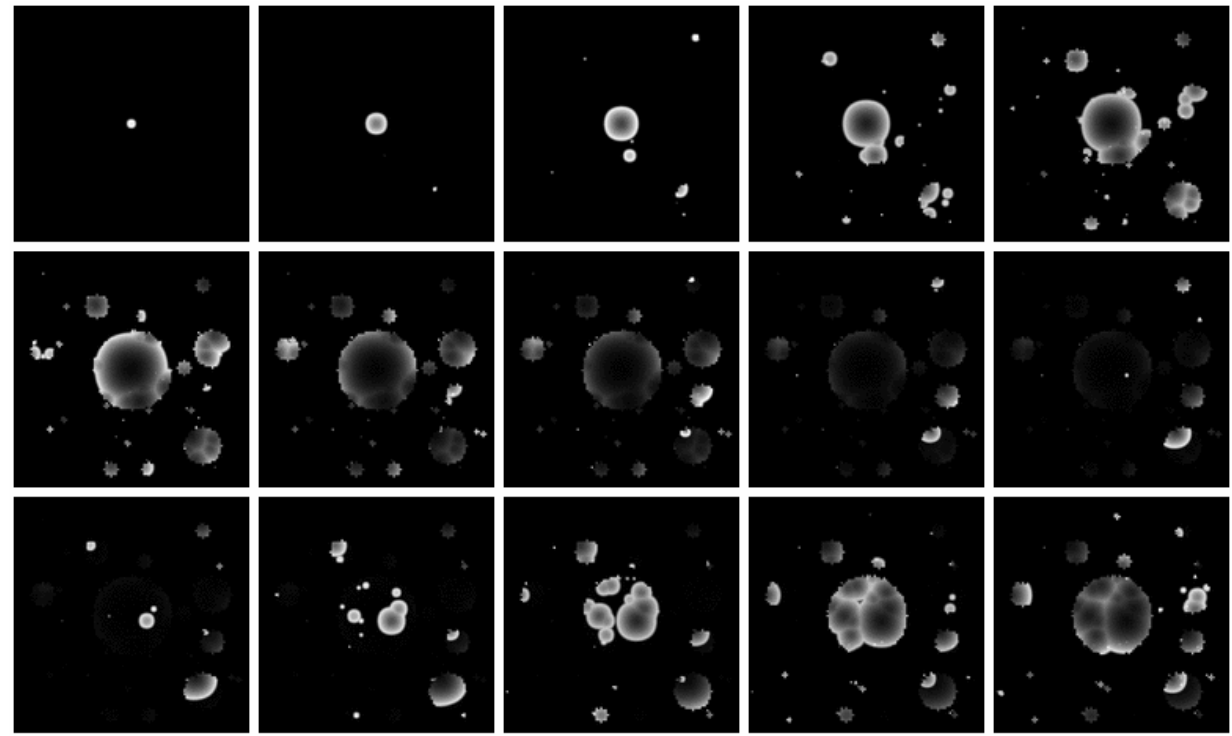

Figure 5.25. Images showing the time sequences of chemical wave propagation with random non-diffusive connections in the domain model in Fig. 5.23 from the numerical integration of the modified Organetor. Infected cells jump to randomly chosen cells in any other domains with the probability of 0.0008. Other conditions as in Fig. 5.24. 


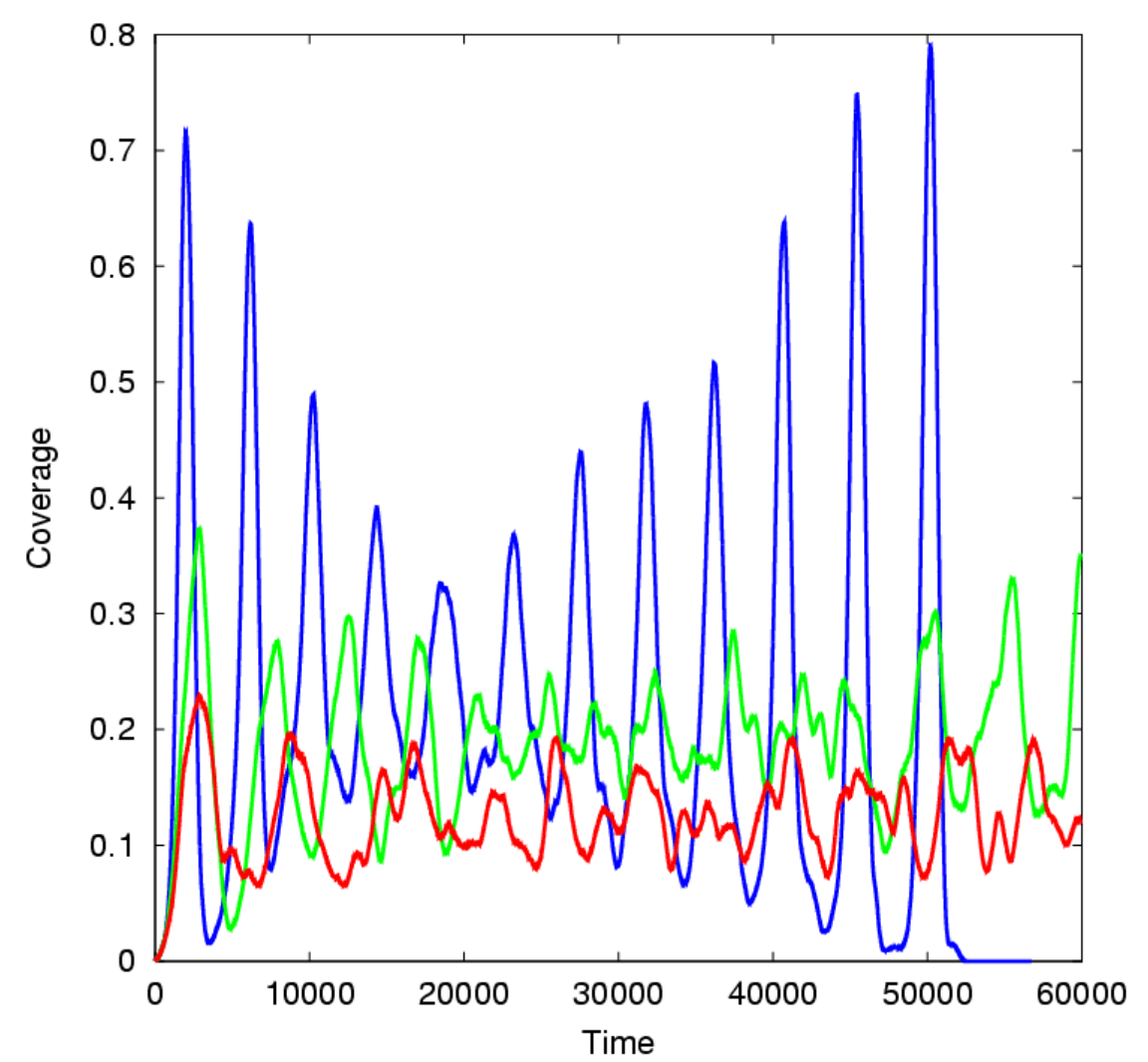

Figure 5.26. Numerical simulations of time-series of fraction of infected elements in domain models. Coverage represents area of $v$, which is the fraction of the area of the oxidized catalyst, $\mathrm{Ru}(\mathrm{bpy})_{3}^{3+}$, in the medium. The jump probabilities are 0.00008 (red), 0.0002 (green) and 0.0008 (blue). Other conditions as in Fig. 5.24. 
All of the studies of modeling disease spreading in networks are either theoretical or numerical simulations. These studies are important in controlling diseases and scientific experiments are needed to obtain information and to test hypotheses. There are examples of forecasting of flu epidemics, as in Ref. [21,22], which are fittings with the actual data and can not be thought as experiments. As stated in Ref. [1], "Experiments in epidemiology with controls are often difficult or impossible to design and even if it is possible to arrange an experiment there are serious ethical questions involved in withholding treatment from a control group." The photosensitive BZ reaction is an interesting alternative model system, as we show here with numerical as well as experimental results.

The non-random long-distance interaction studied in the static networks is suitable to mimic a short-term and daily mobility in relatively isolated populations. In Ref. [77], the authors found that "travel within the island of Dominica is clearly nonrandom" by analyzing the travel data obtained from interviewing 305 individuals from all parts of the island about their daily travel patterns, their travel off the island, and the travel of members of their immediate family. Those mobility data were linked with data on the patterns of measles transmission during a 1984 epidemic. We can add static links in a domain model network to mimic the non-random travel in geographically separated regions to compare with the real disease data.

In both dynamic and static networks, we find that the time series of the fraction of excited elements change from irregular fluctuations with small amplitude to regular self-sustained oscillations with large amplitude as the jump probability or the number of static links is increased. A pronounced global synchronization of the excitated elements, simulating the infected population, was induced as the jump probability increases in dynamic networks. Similar simulation results were reported in modeling disease spreading on one-dimensional small-world networks with the SIRS model [39]. Epidemiologically, this situation resembles periodic epidemic patterns [78]. For example, the Black Death reoccurred regularly in various parts of Europe for more 
than 300 years after it arrived from Asia during the fourteenth century [17]. Periodic epidemic infection is part of the reason of population changes throughout the world in the eighteenth century [1]. It has been reported that measles epidemics in UK cities were regular with high spatial synchronization as 2-year outbreak before the mass vaccination [79] and chooping cough synchronized at 3.5-year outbreaks across England and Wales after the vaccine era [79].

Domain model networks provide examples of geographic city distributions in the context of a large country (or even the entire earth) with some cities having different sizes and a good transportation system between them. The movements from one city to another are rapid, and the eventual propagation of an epidemic takes place only at destination locations. The situation is much like that of a directed network with different sizes nodes, such as the structure of the internet [32]. In this sense, it might also be suitable to mimic computer viruses spreading in the internet through emails. As we pointed out, the static links can also be used in domain models. We can define the "source domain" and "destination domain" as well as the "source cell" and "destination cell." An interesting comparison will be with the model in Ref. [23], with the population divided in seven districts, each of which was further divided into three age classes.

The results in these three different networks increase our understanding of the role that long-distance interactions play in infectious disease spreading. The insights that arise from such studies will aid in our ability to predict the course of future epidemics, which will allow for better strategies to combat the spread of epidemics in human and animal populations as well as the spread of computer virus in the Internet. 


\subsection{Appendix A: The Program in OPTIMAS Used for Dynamic Networks in the Experiments}

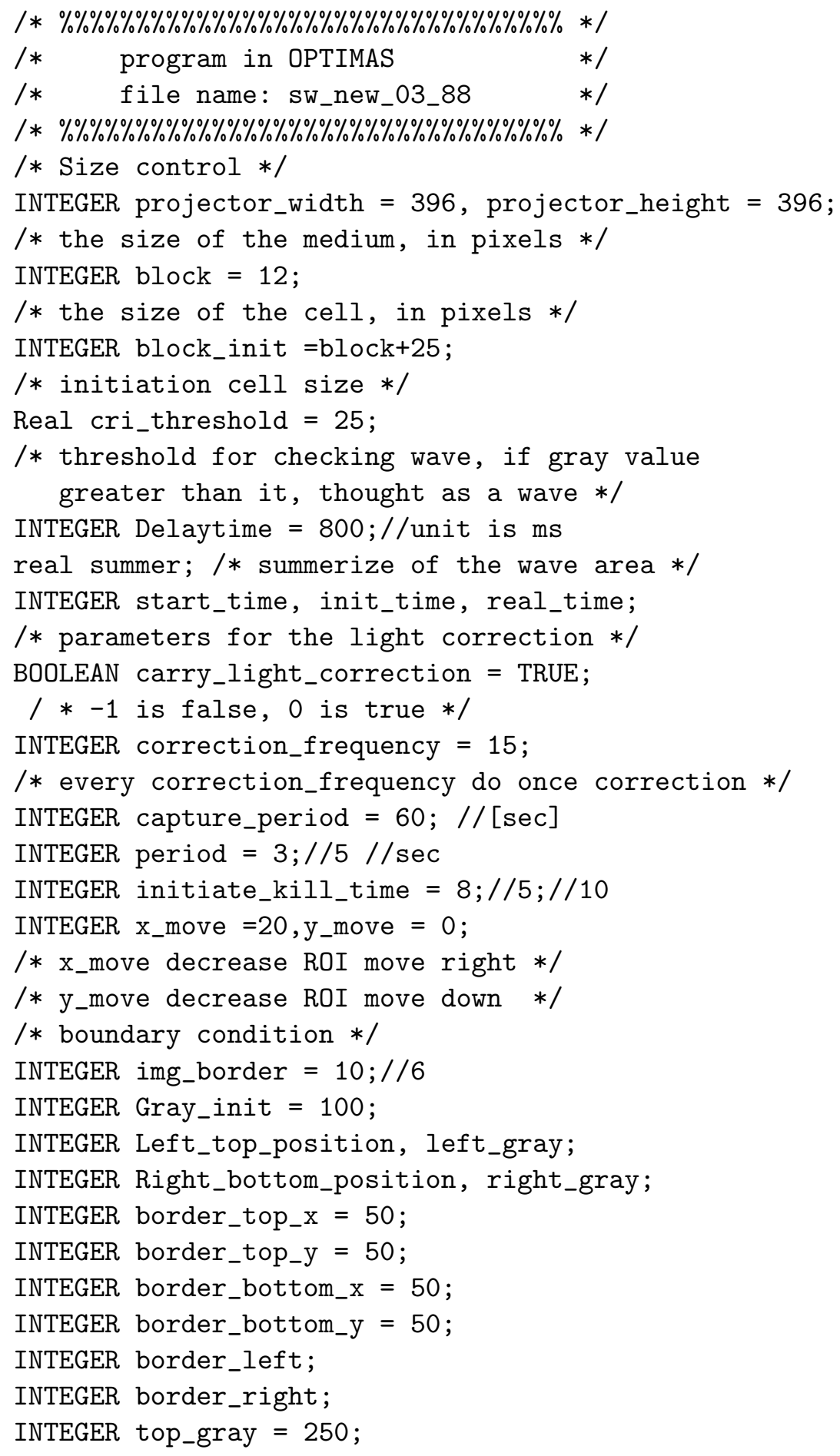




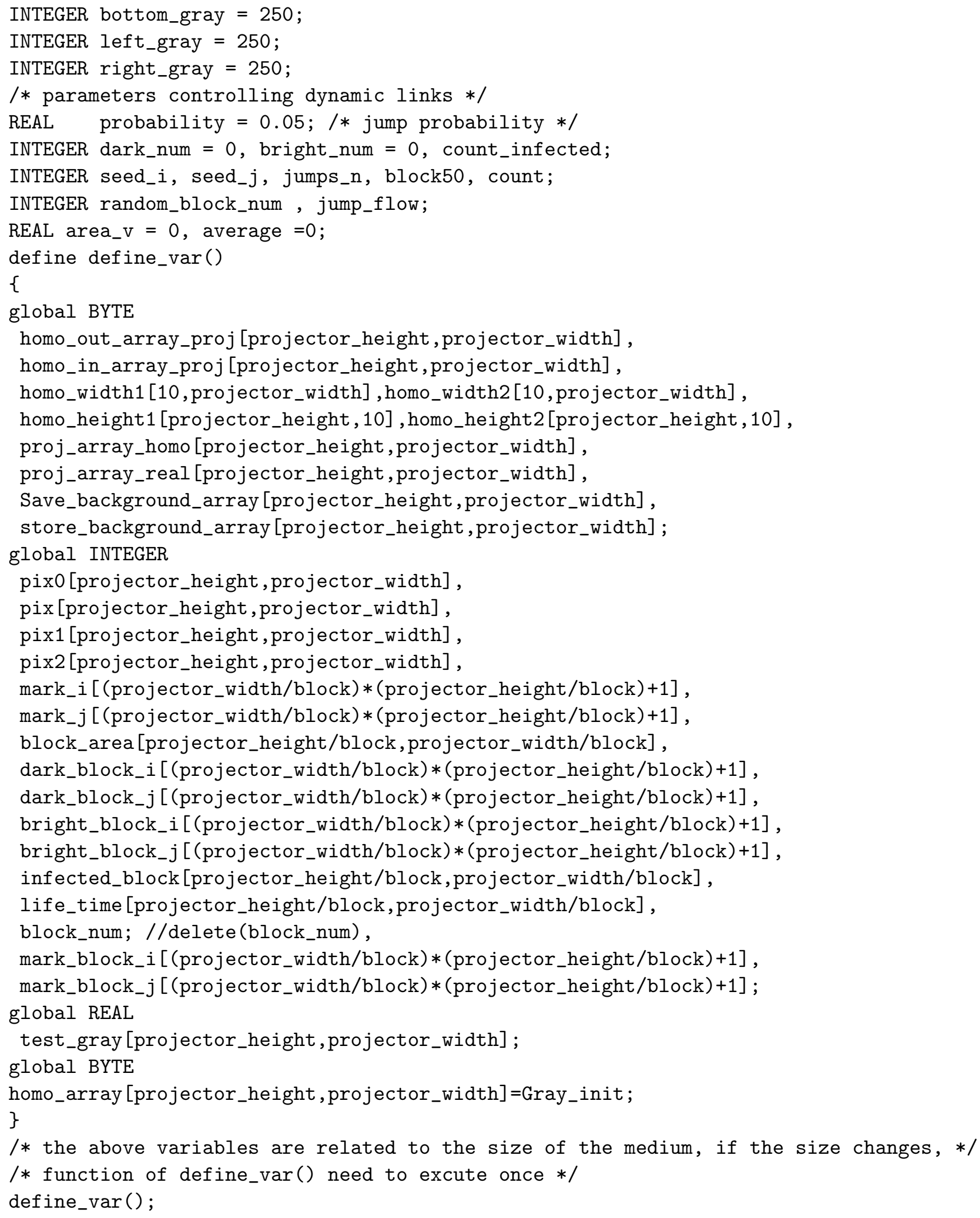




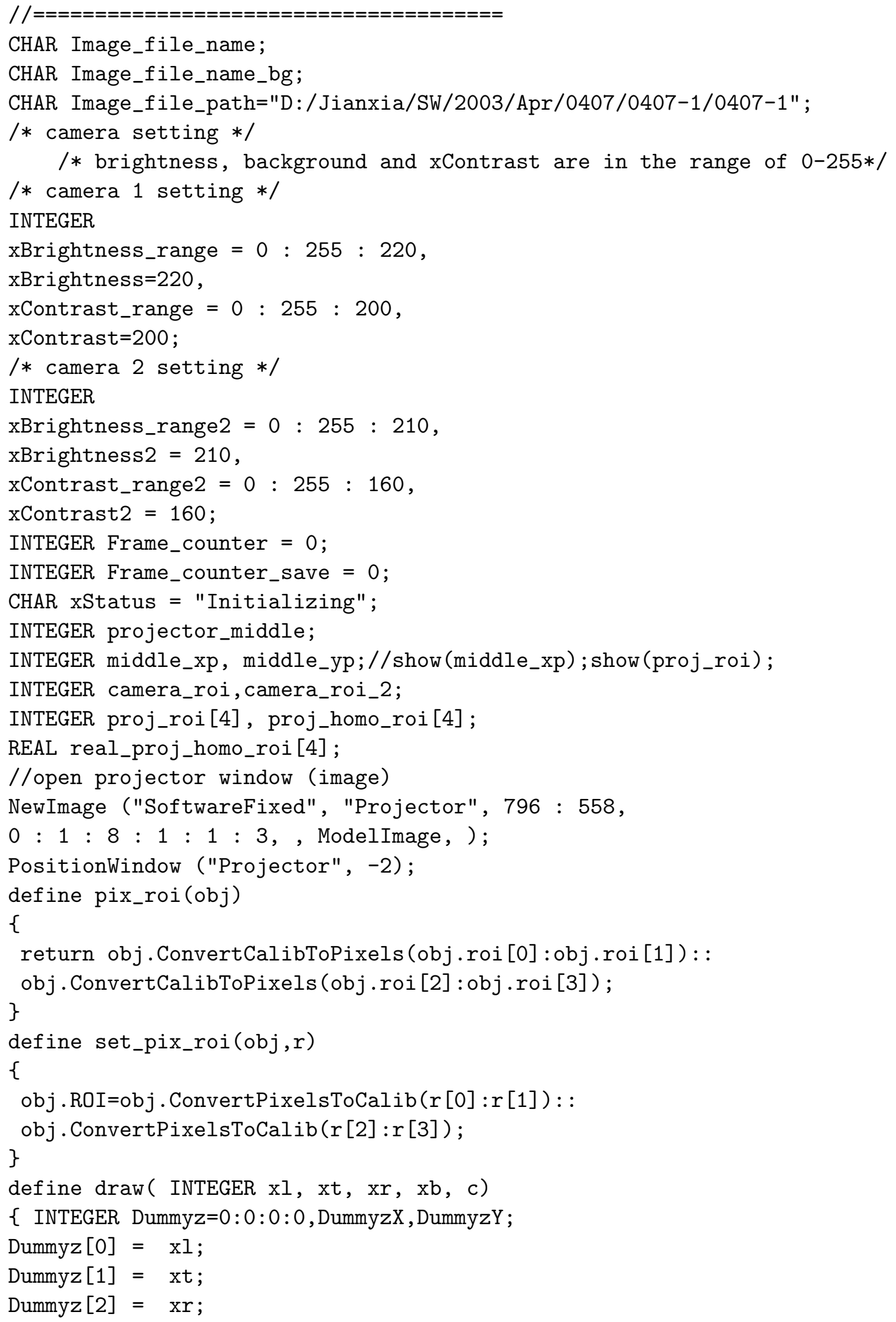




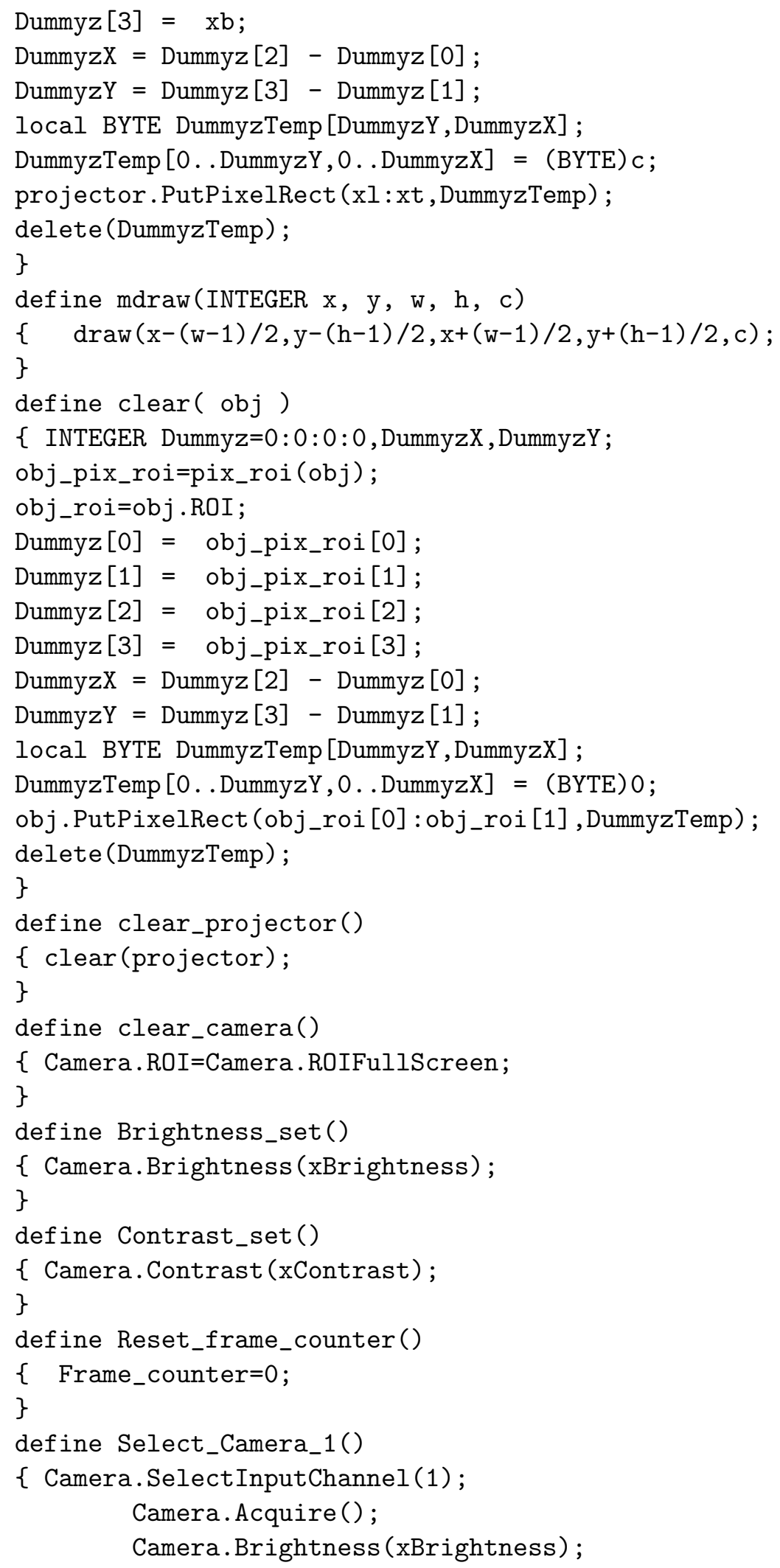




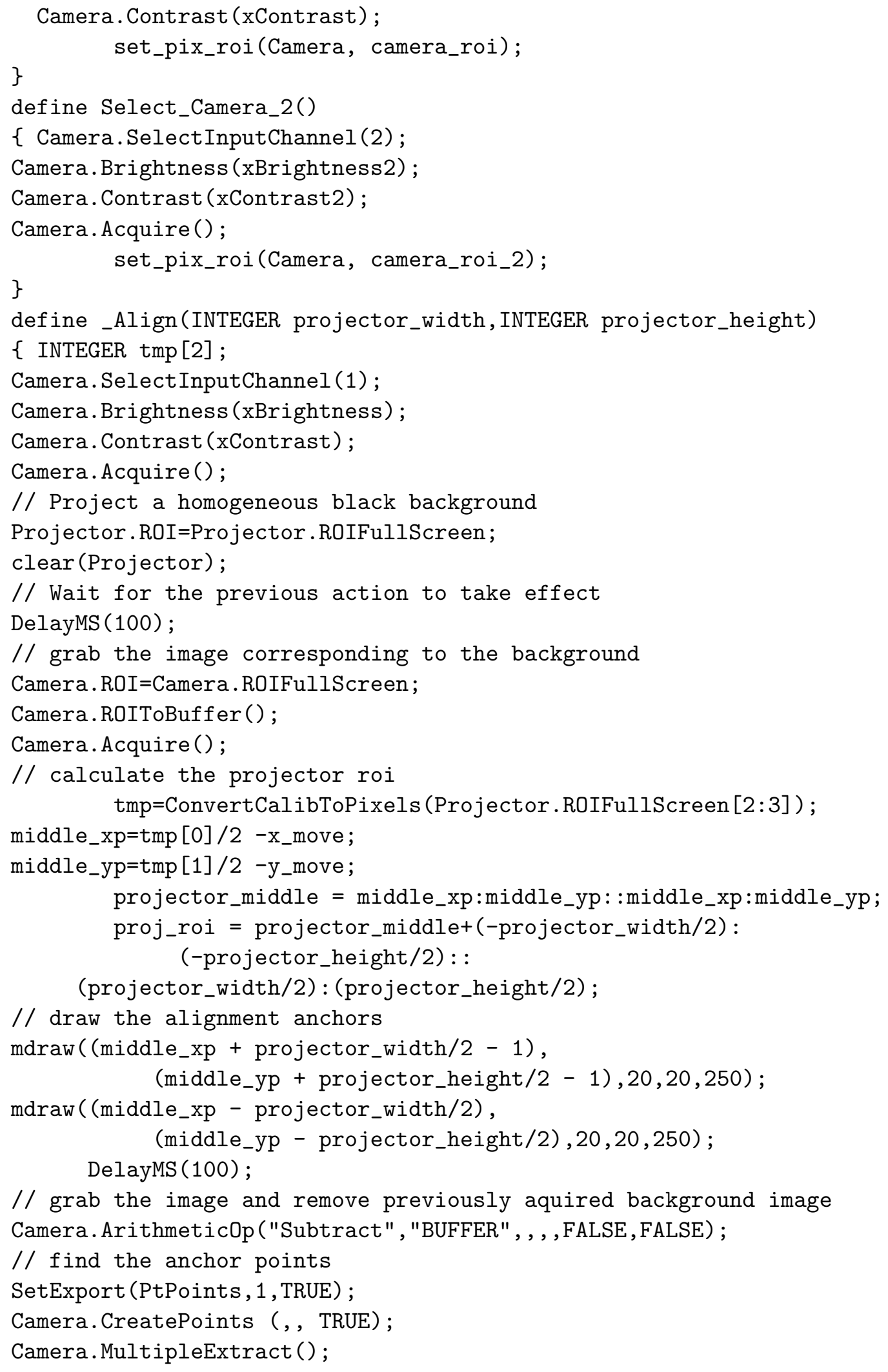




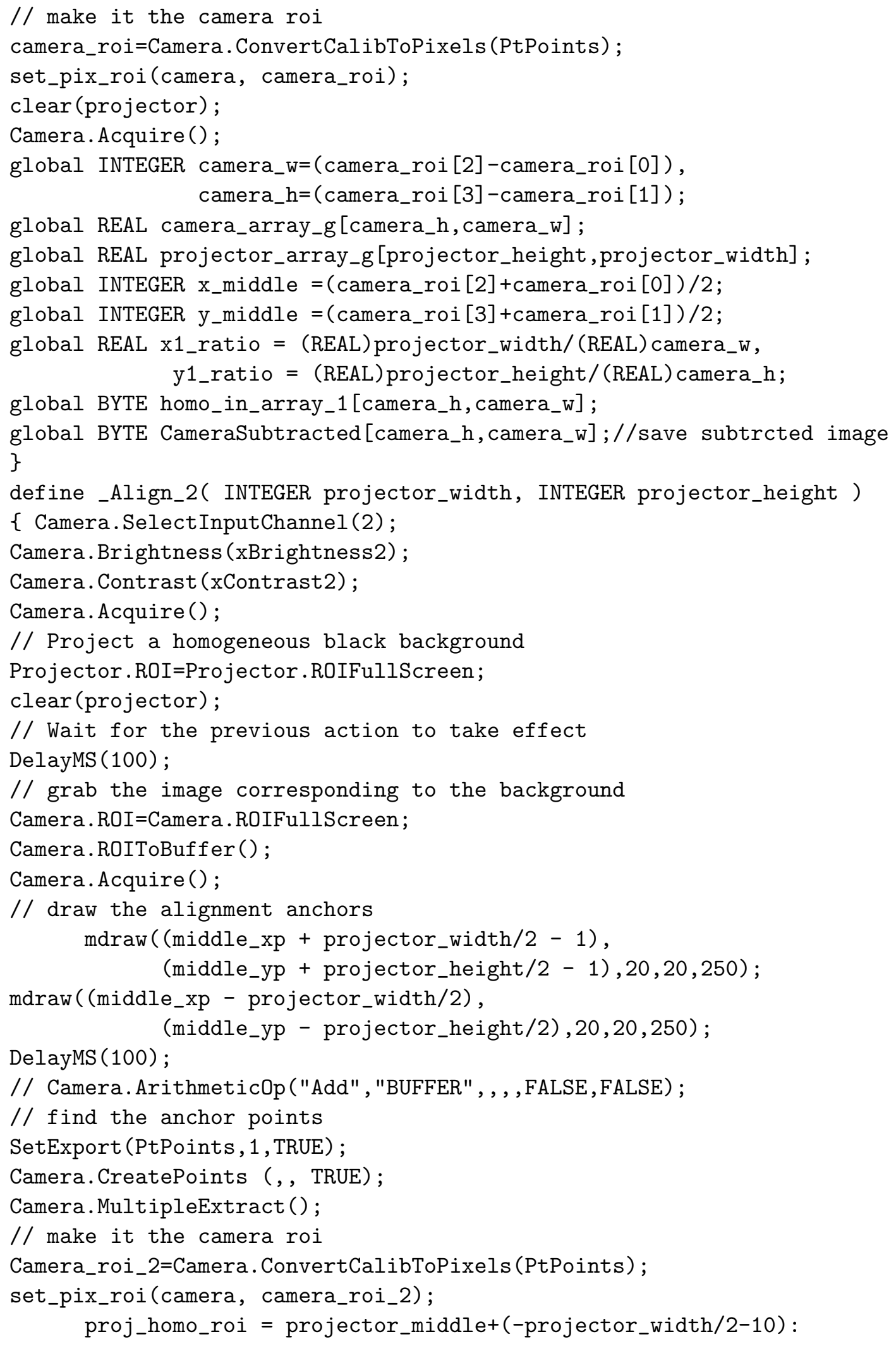




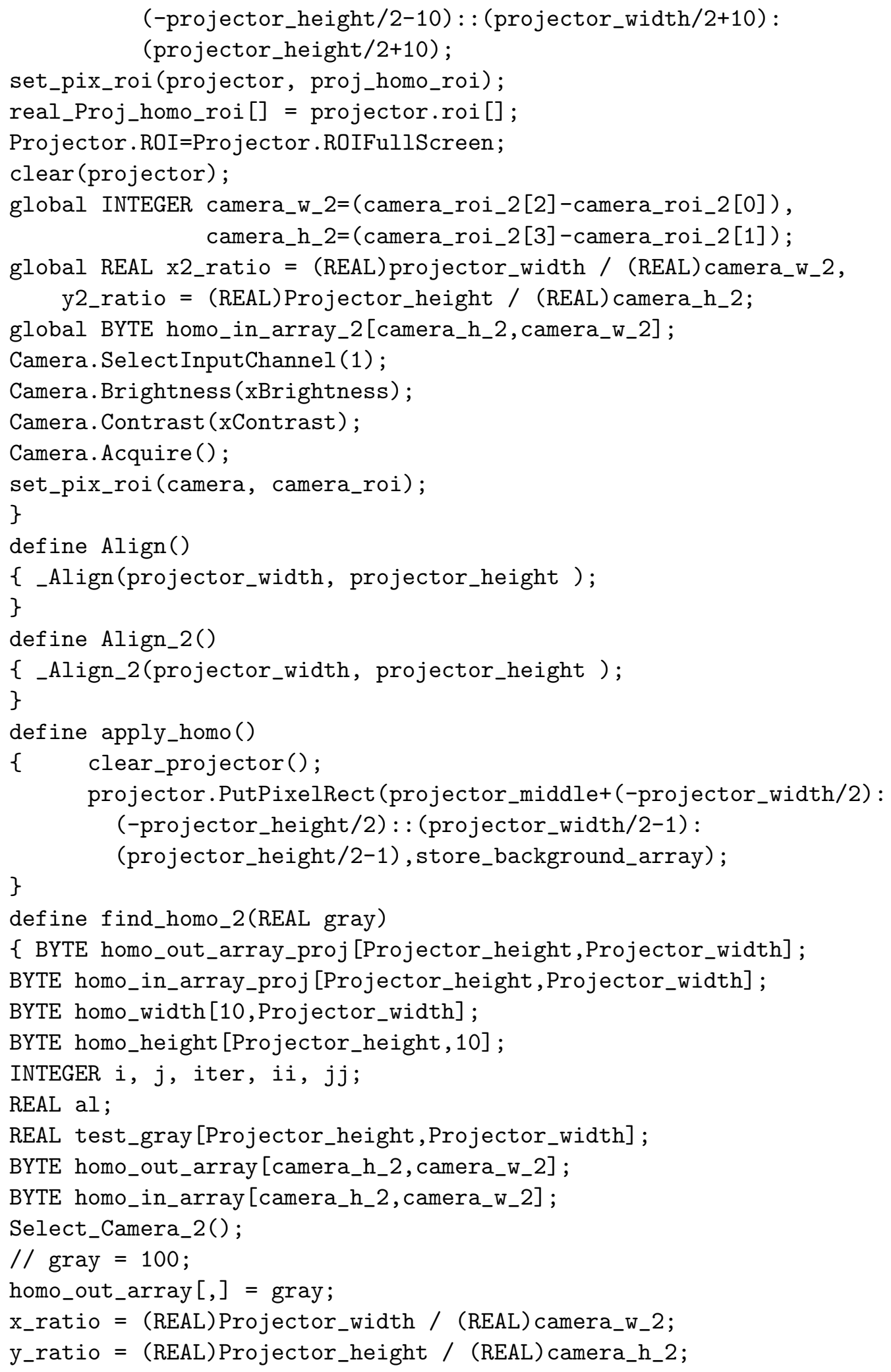




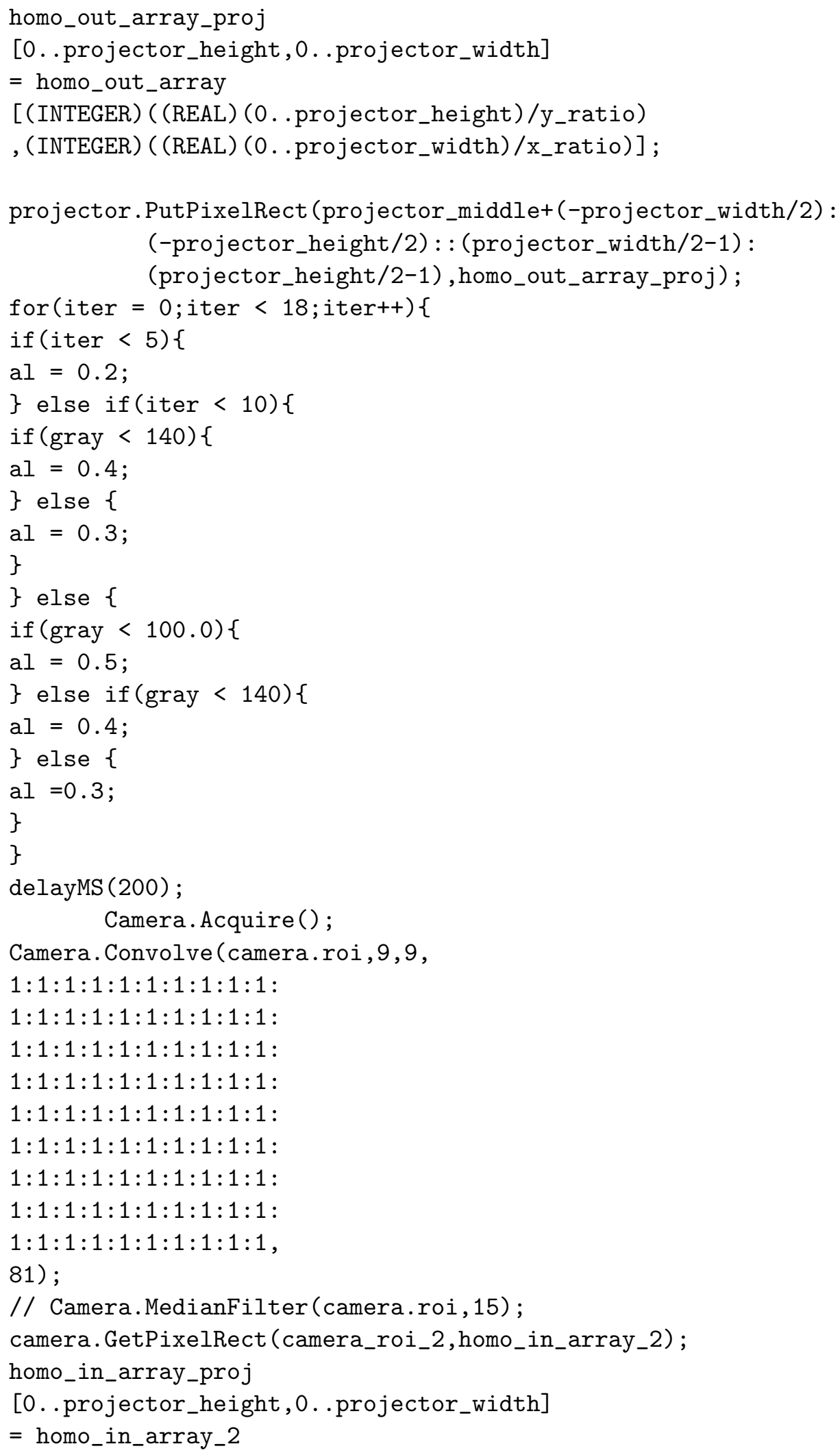




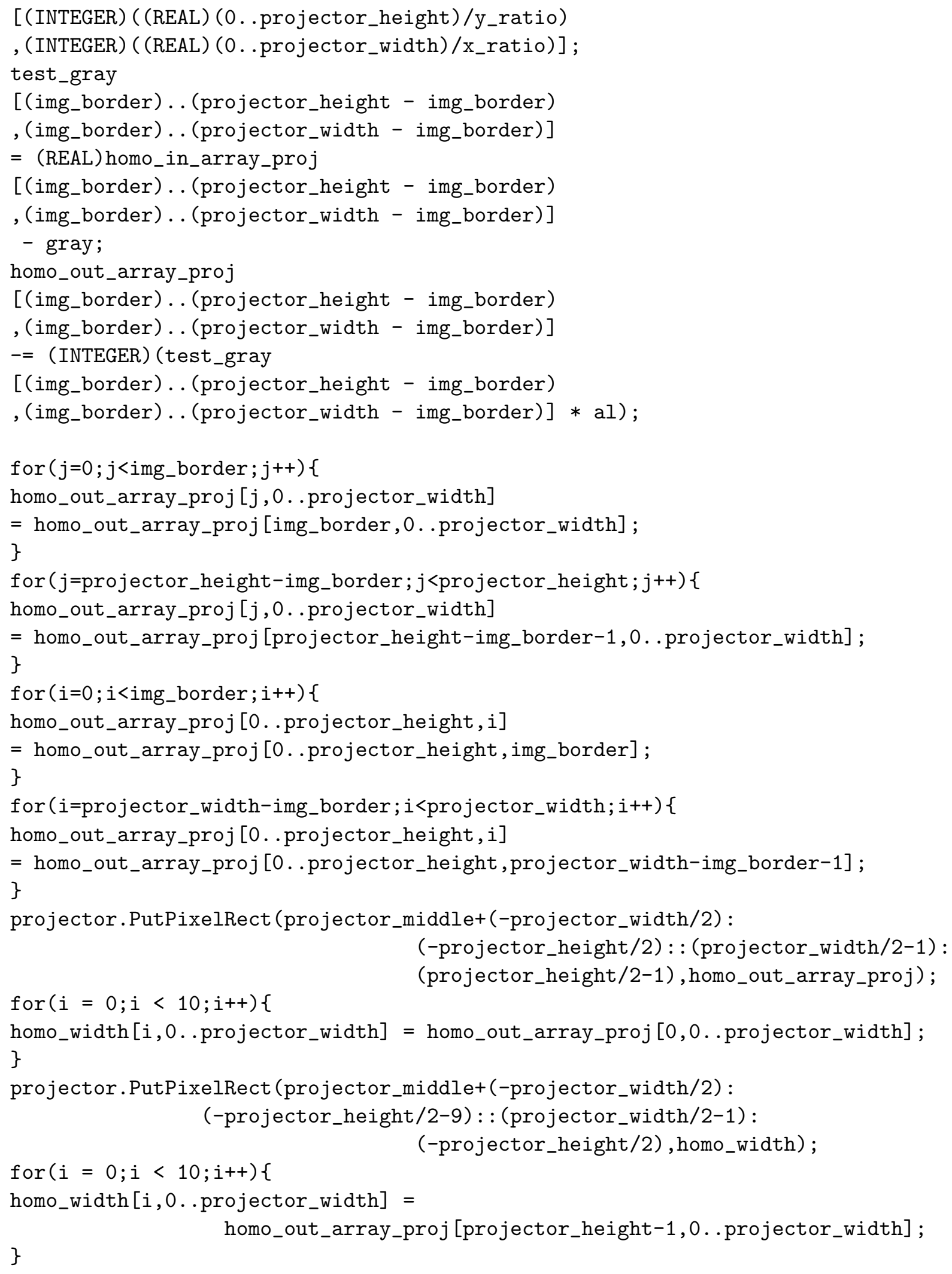




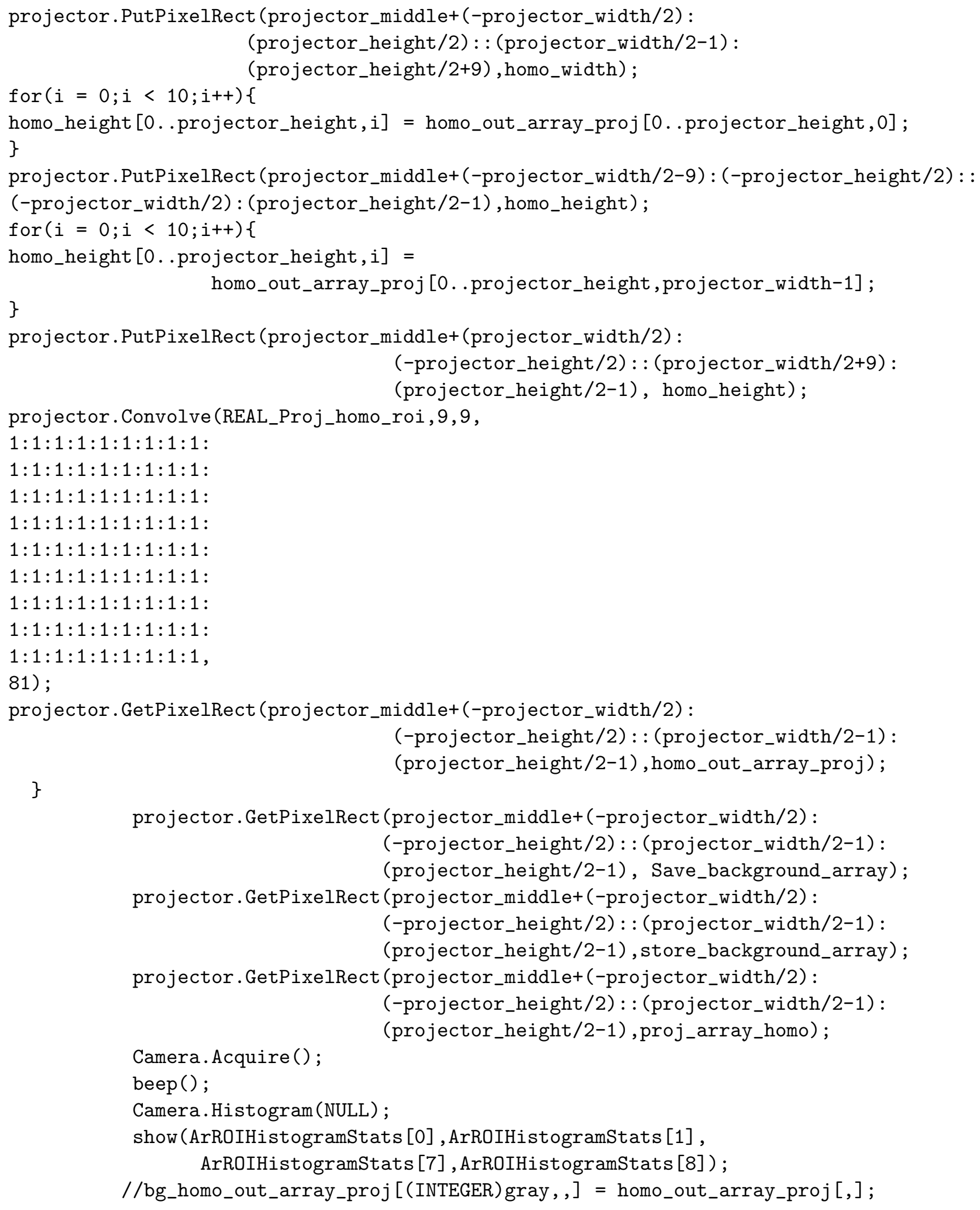




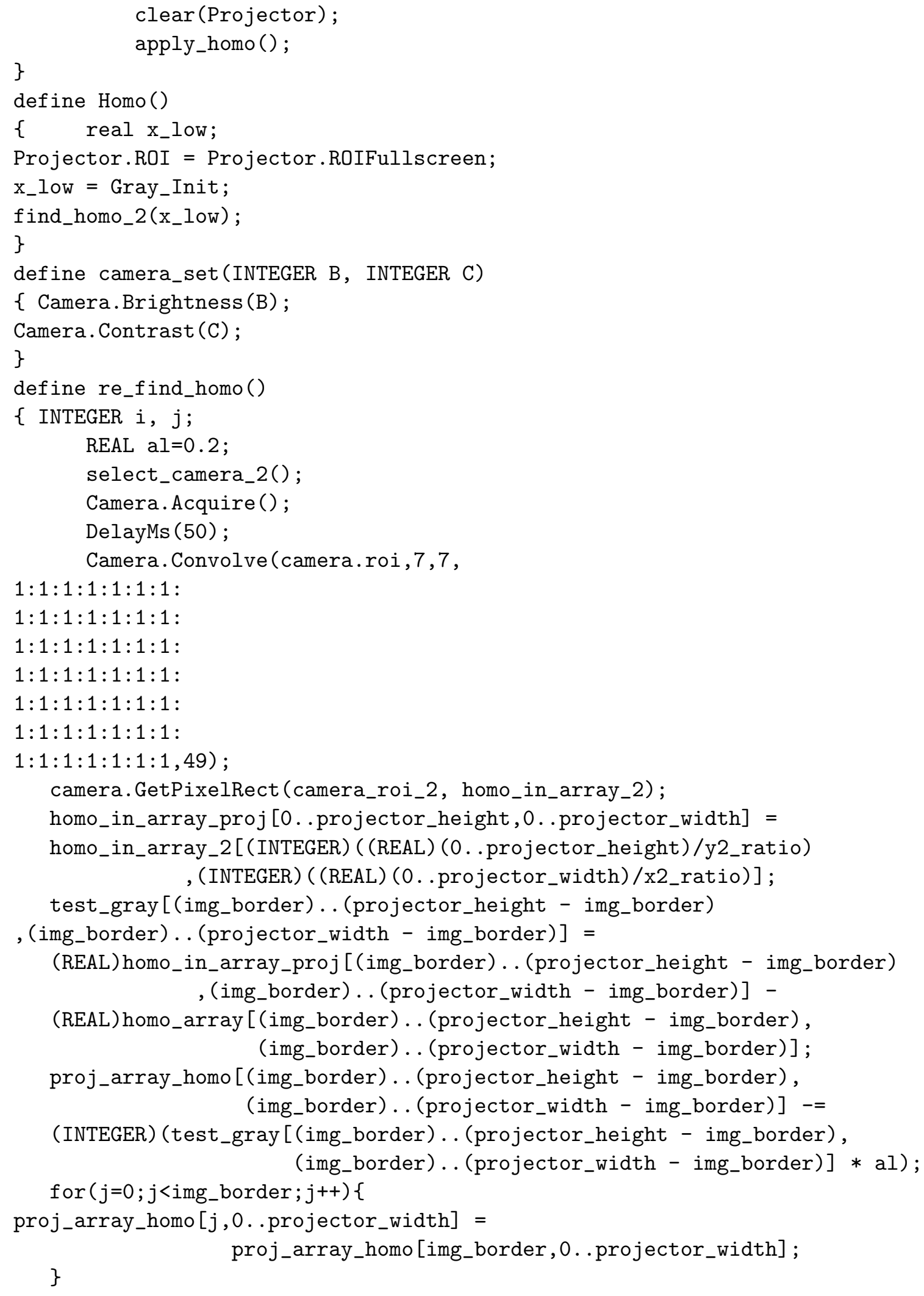




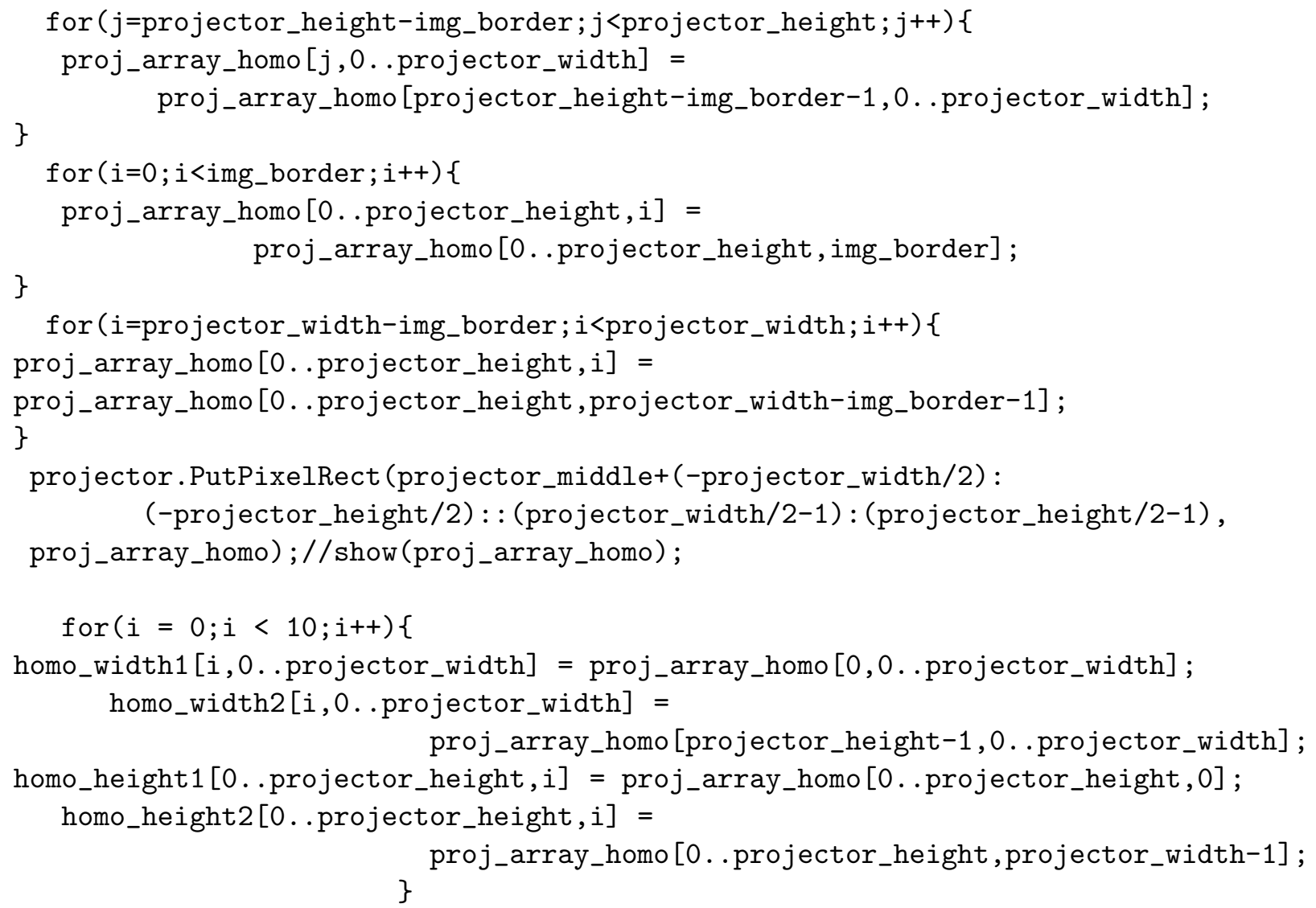




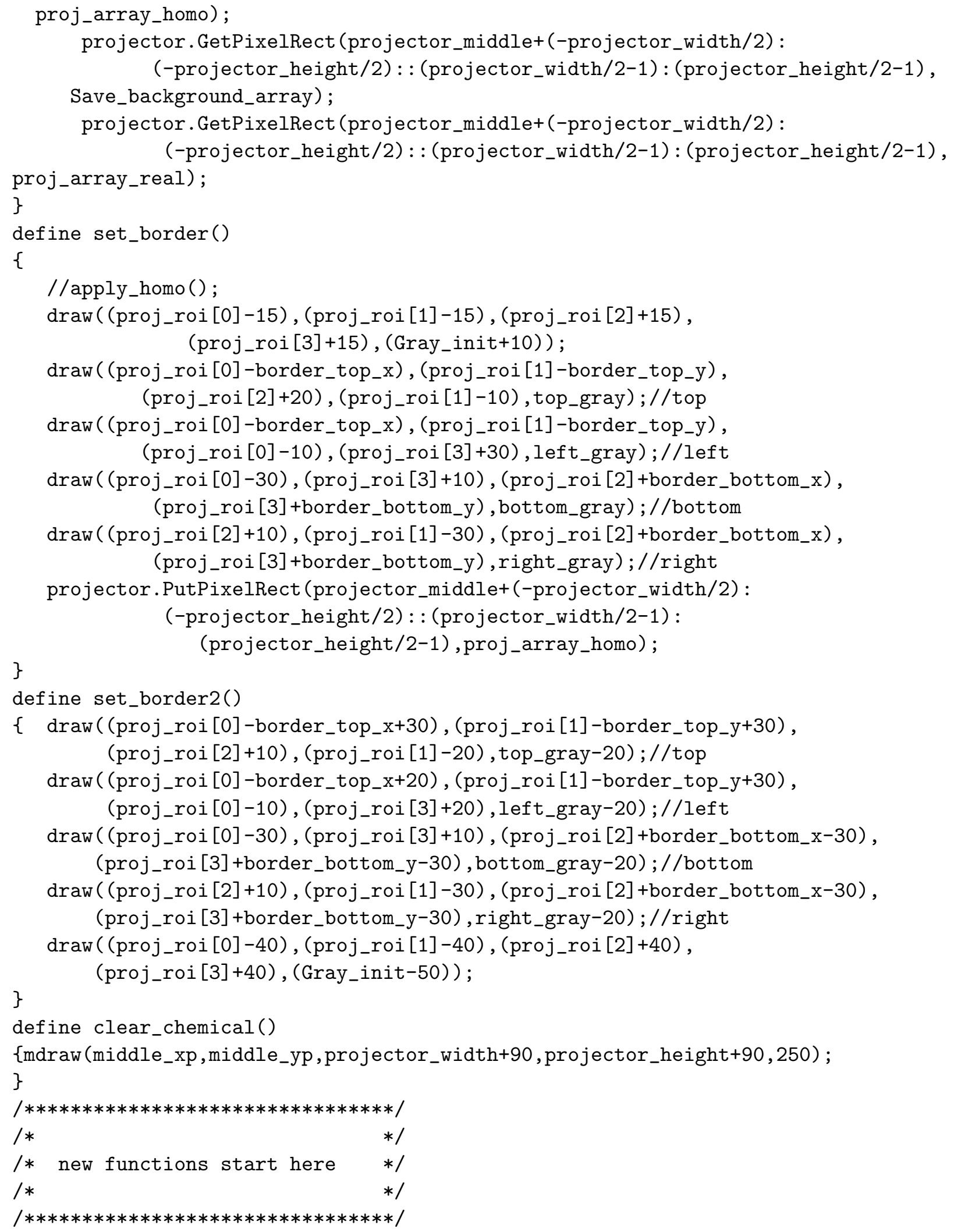




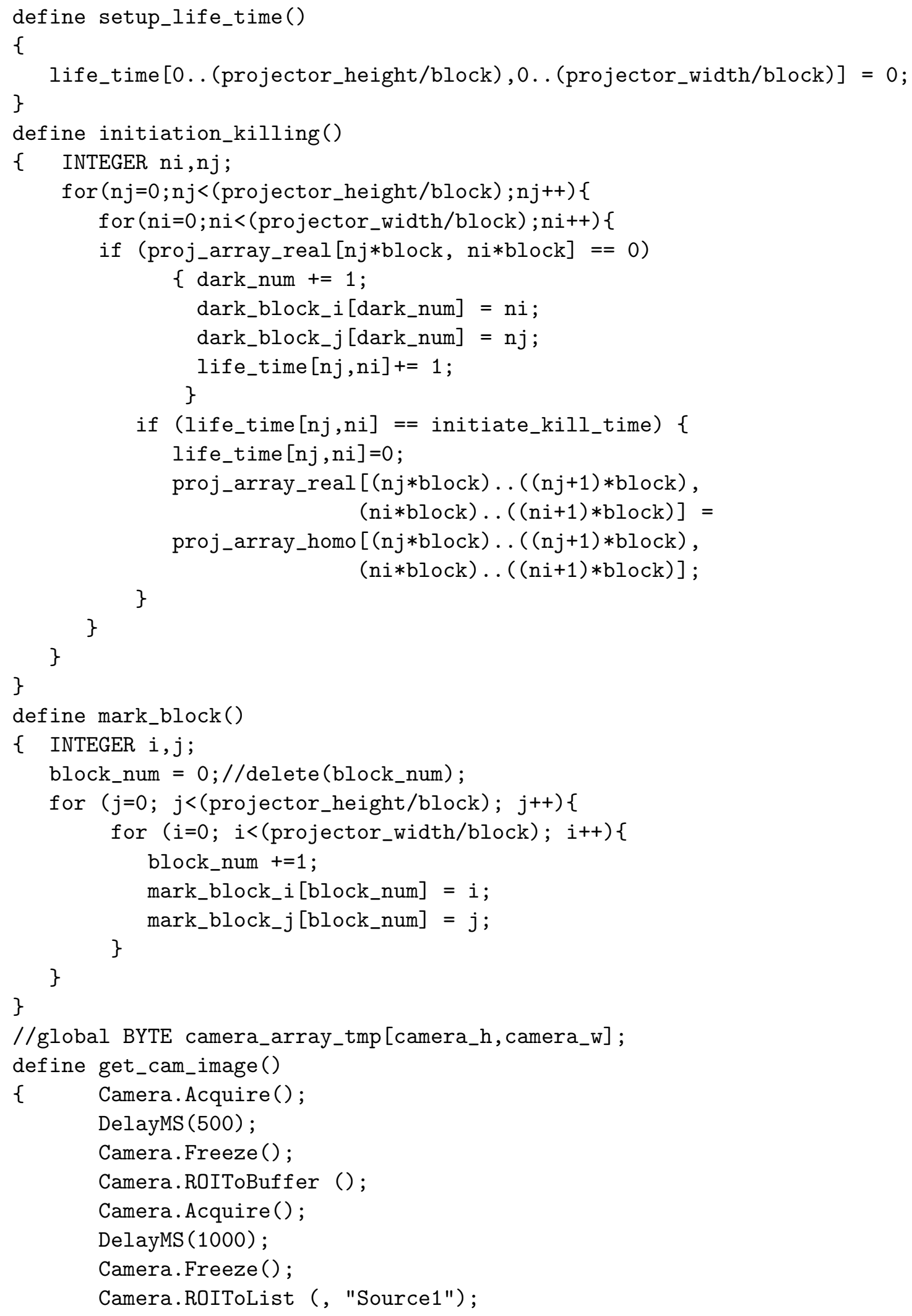




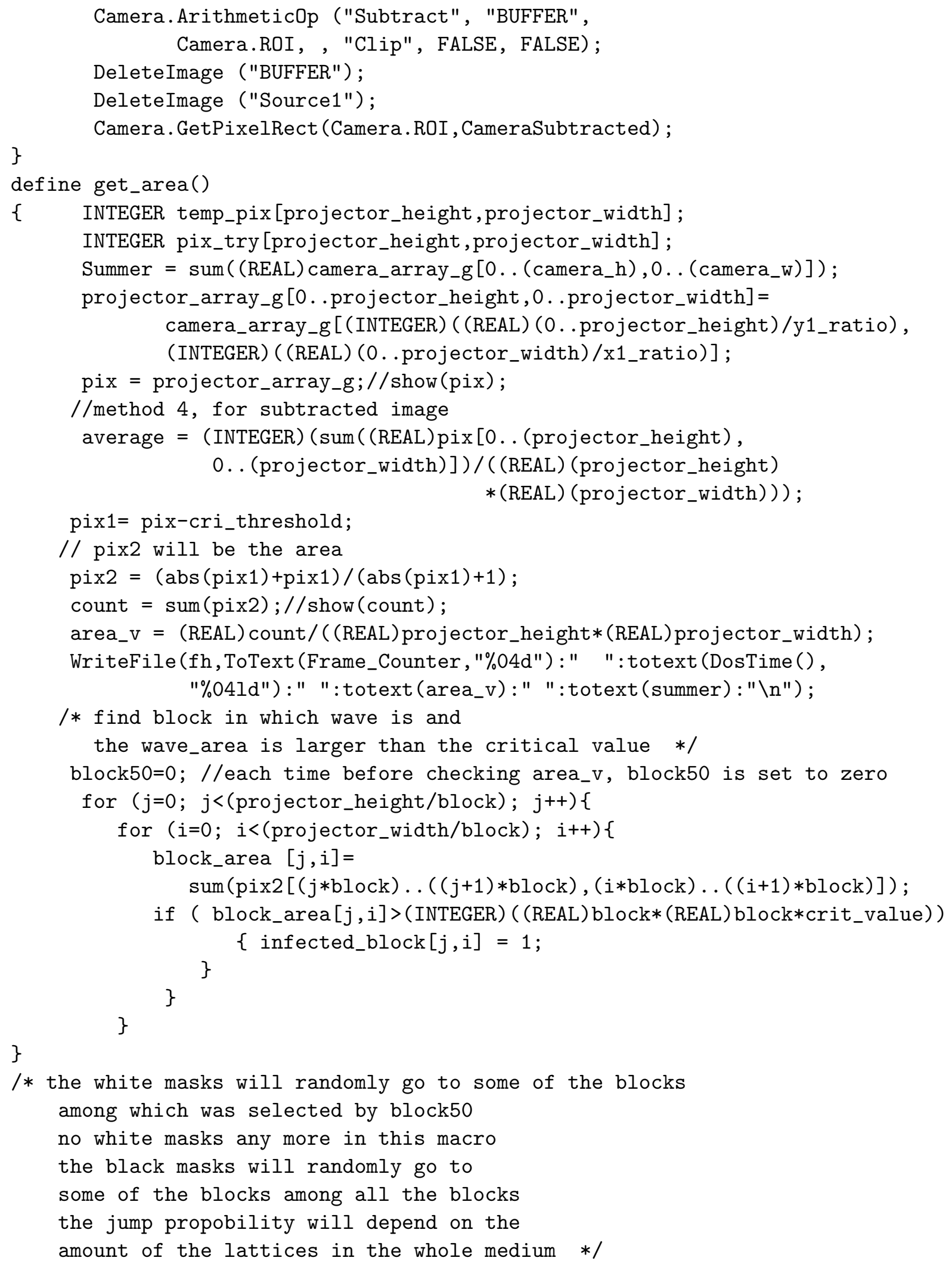




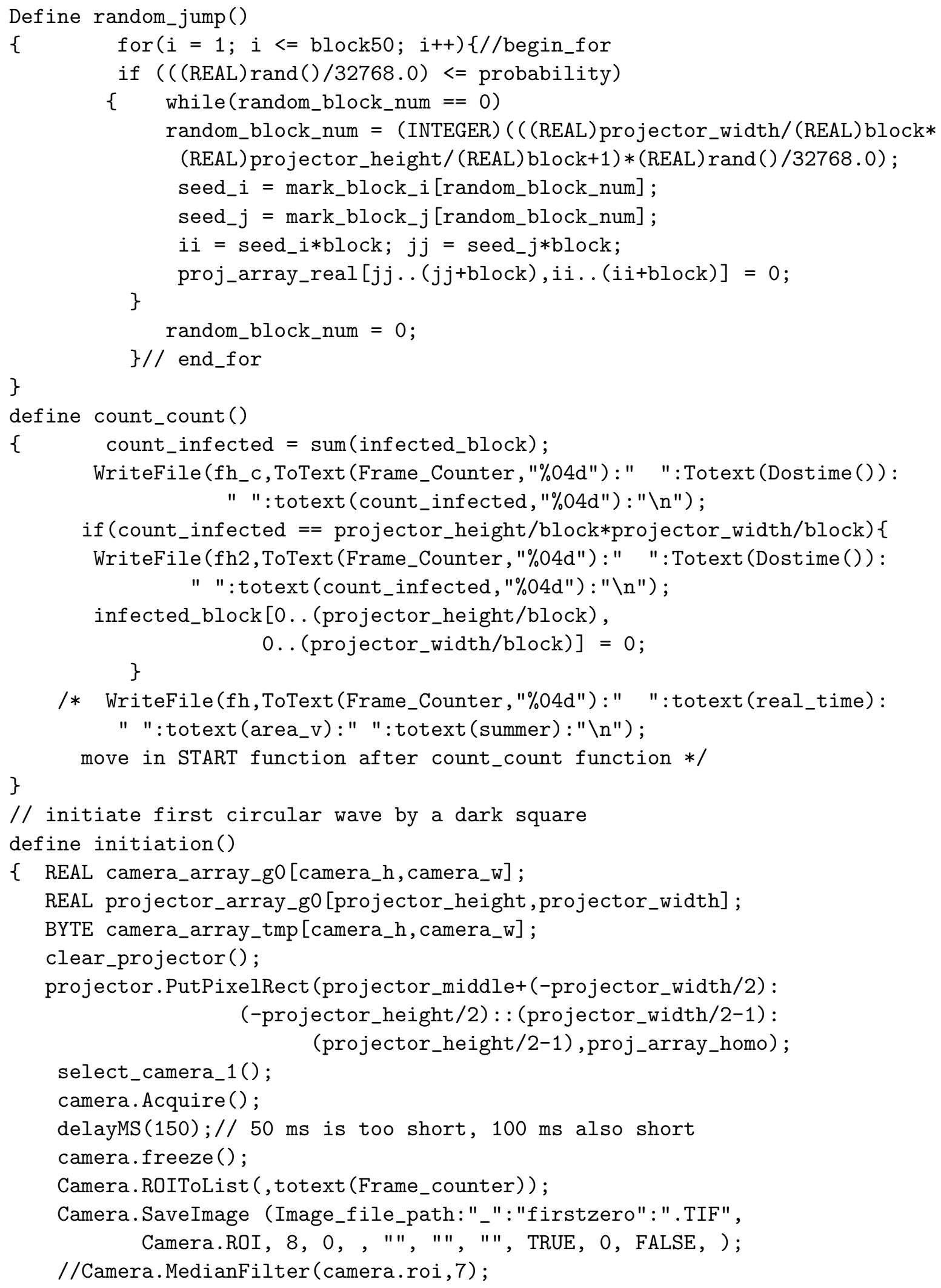




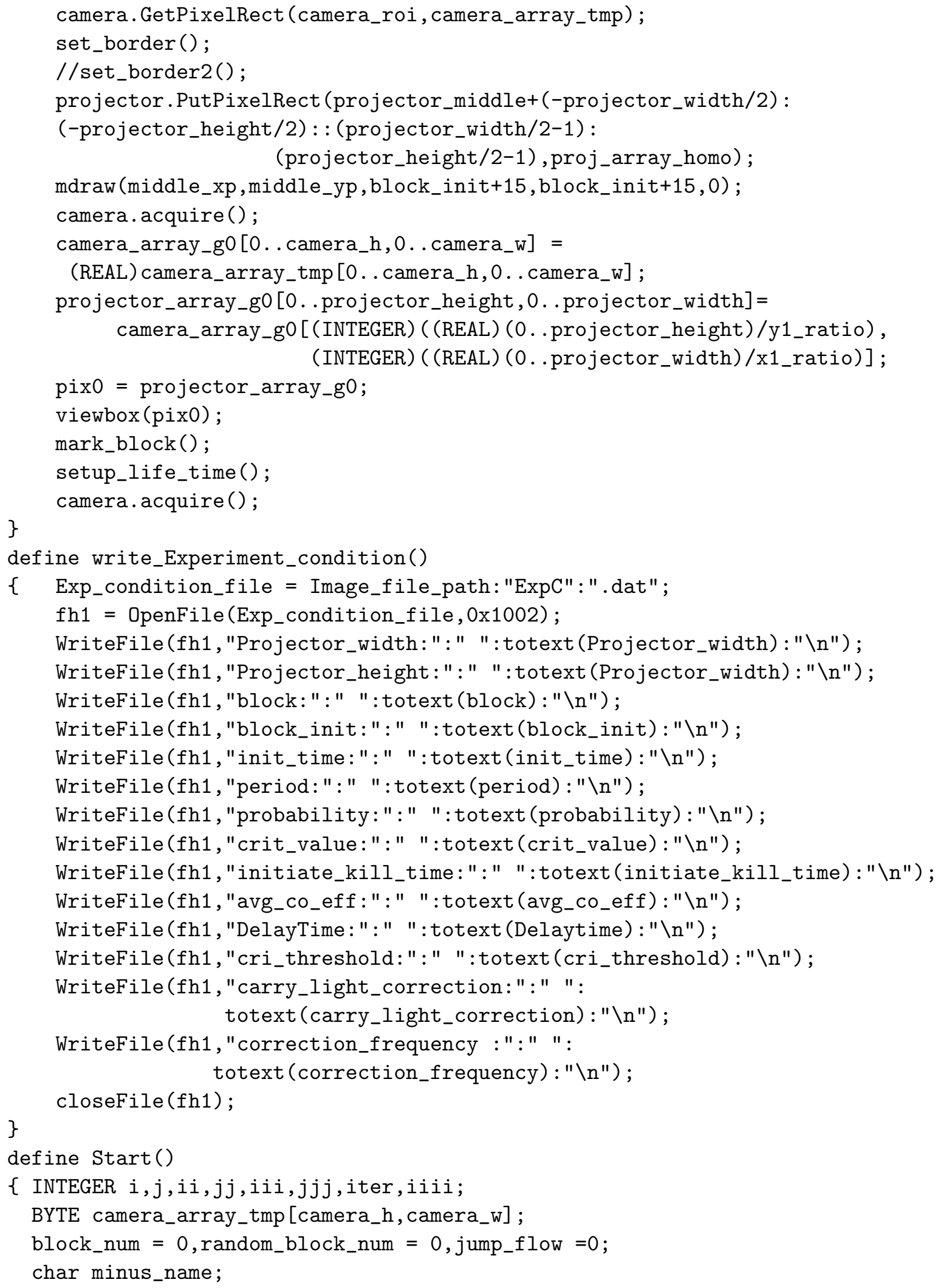




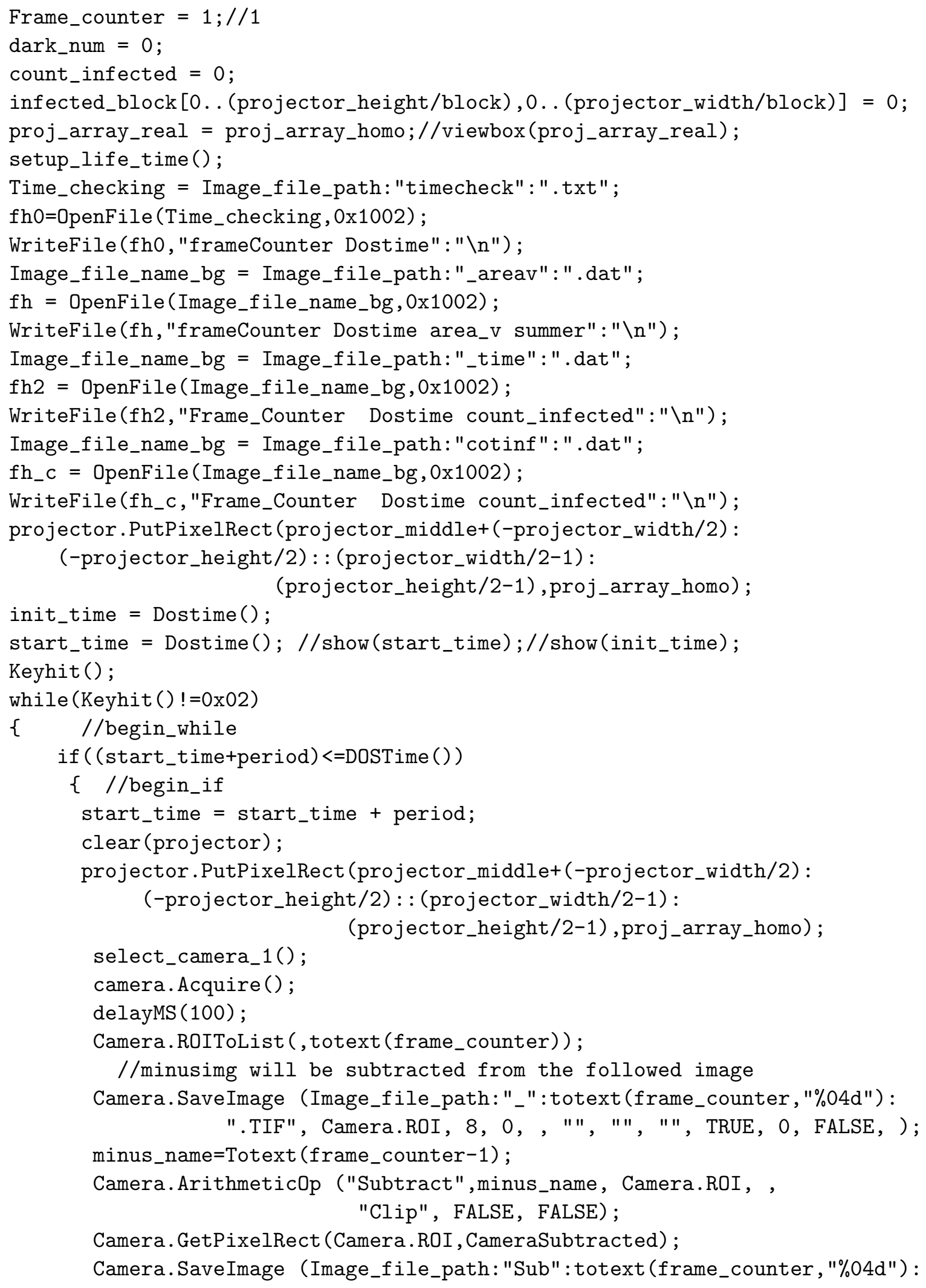




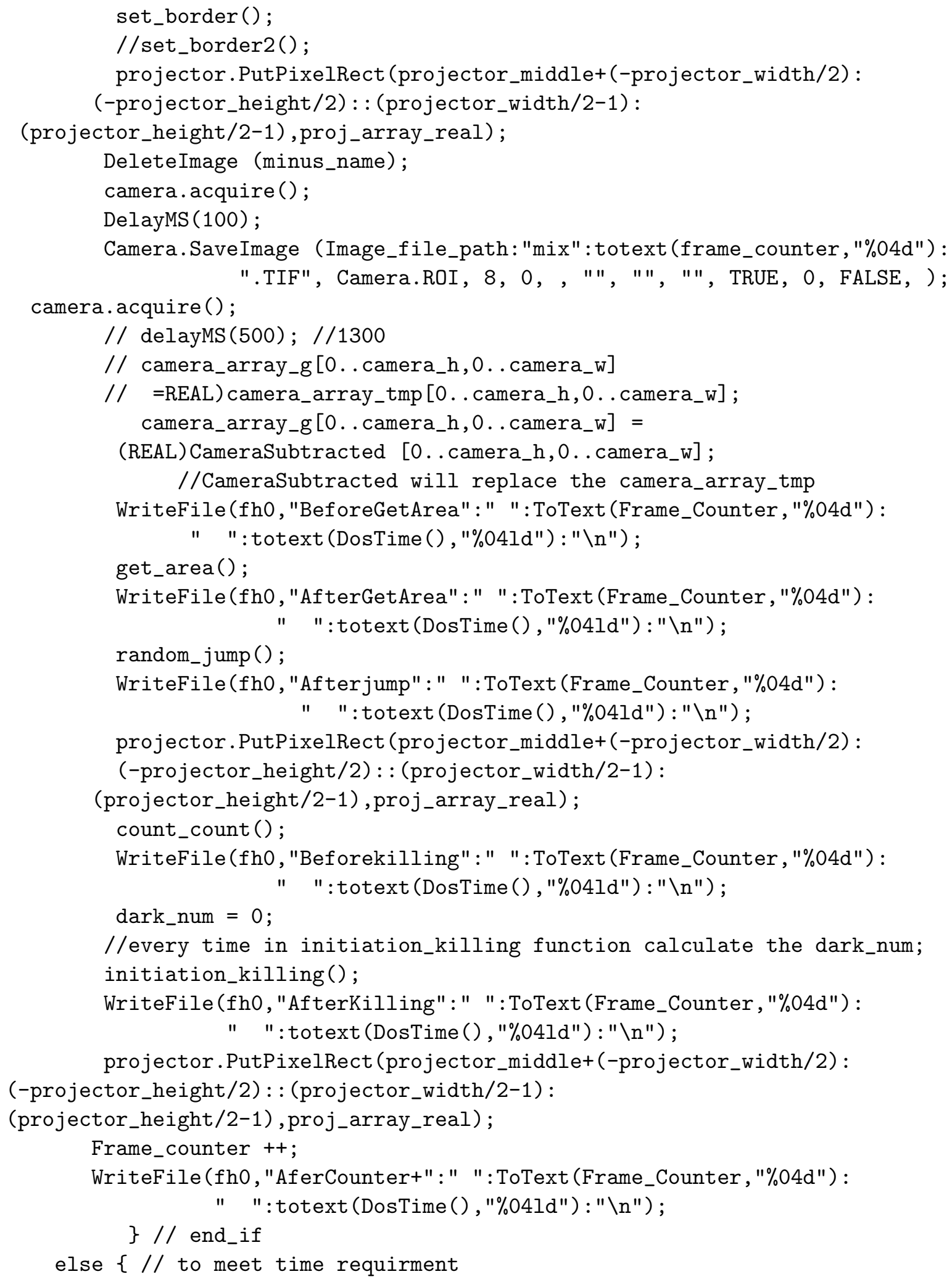




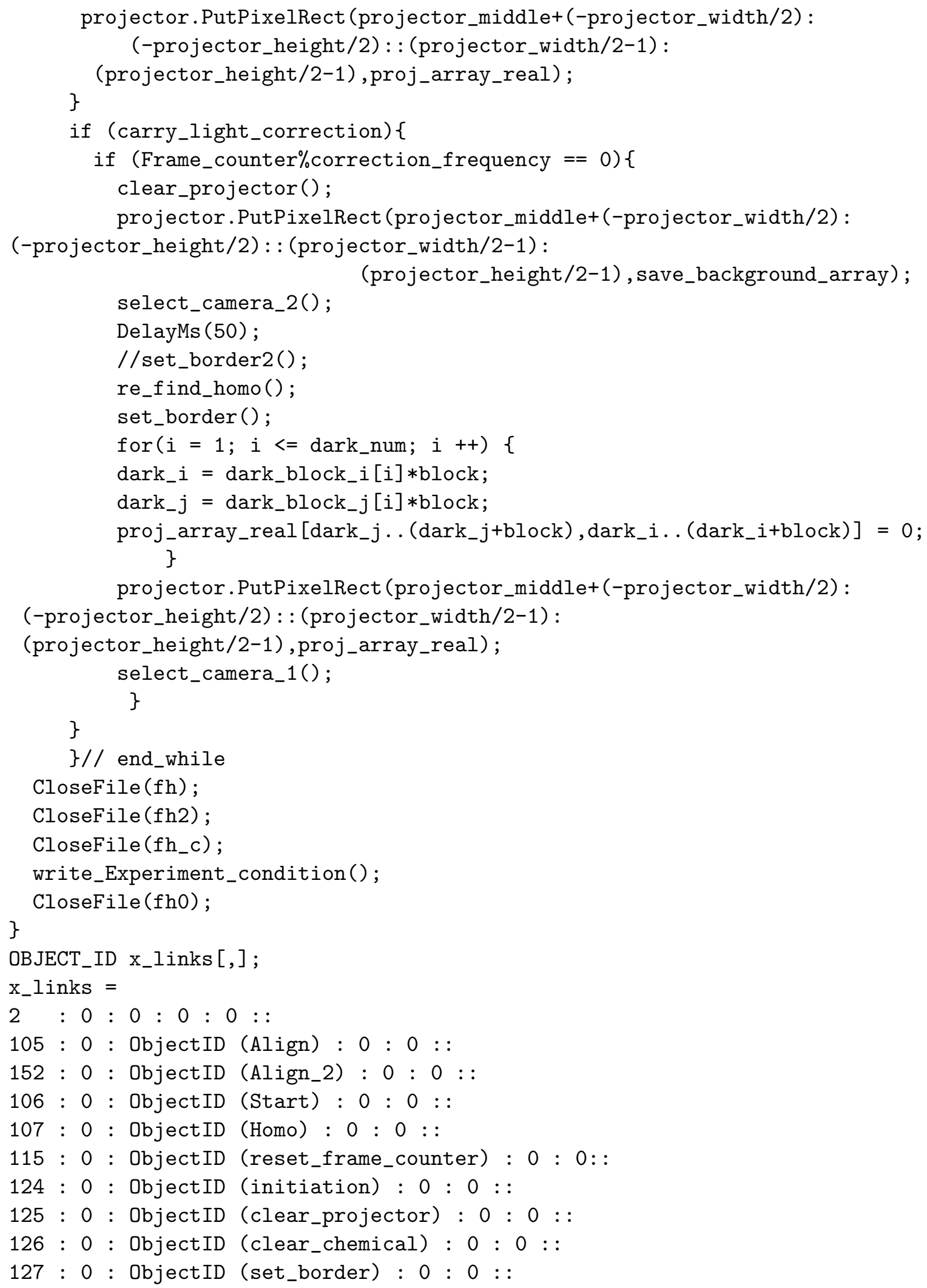




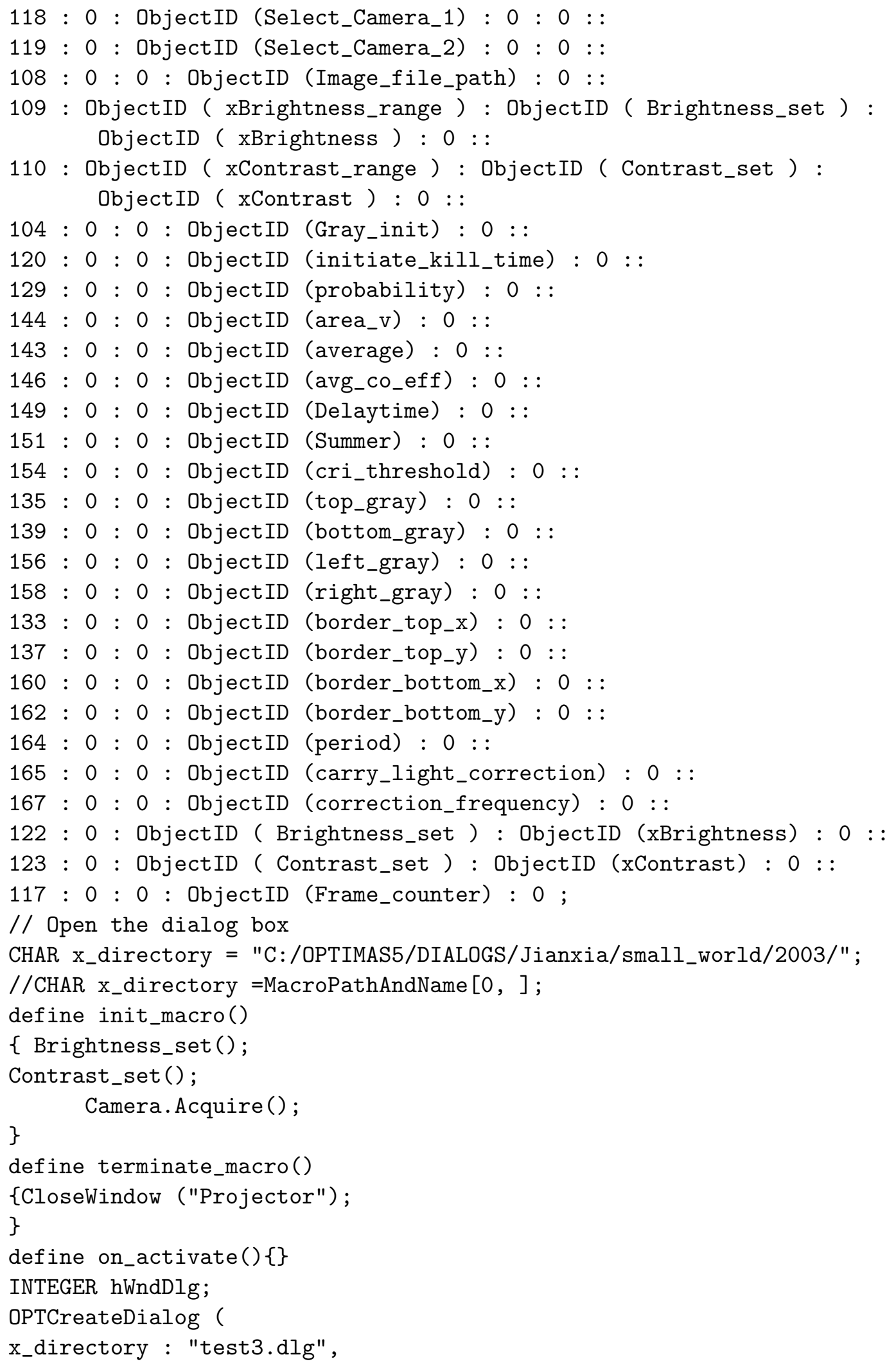




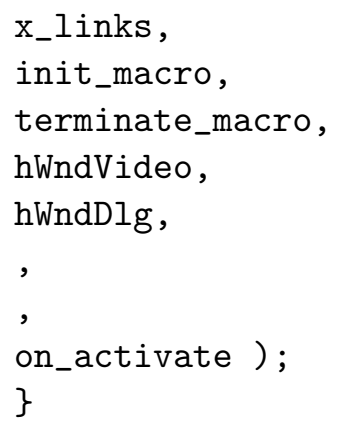

\subsection{Appendix B: The Program in OPTIMAS Used for Static Networks in the Experiments}

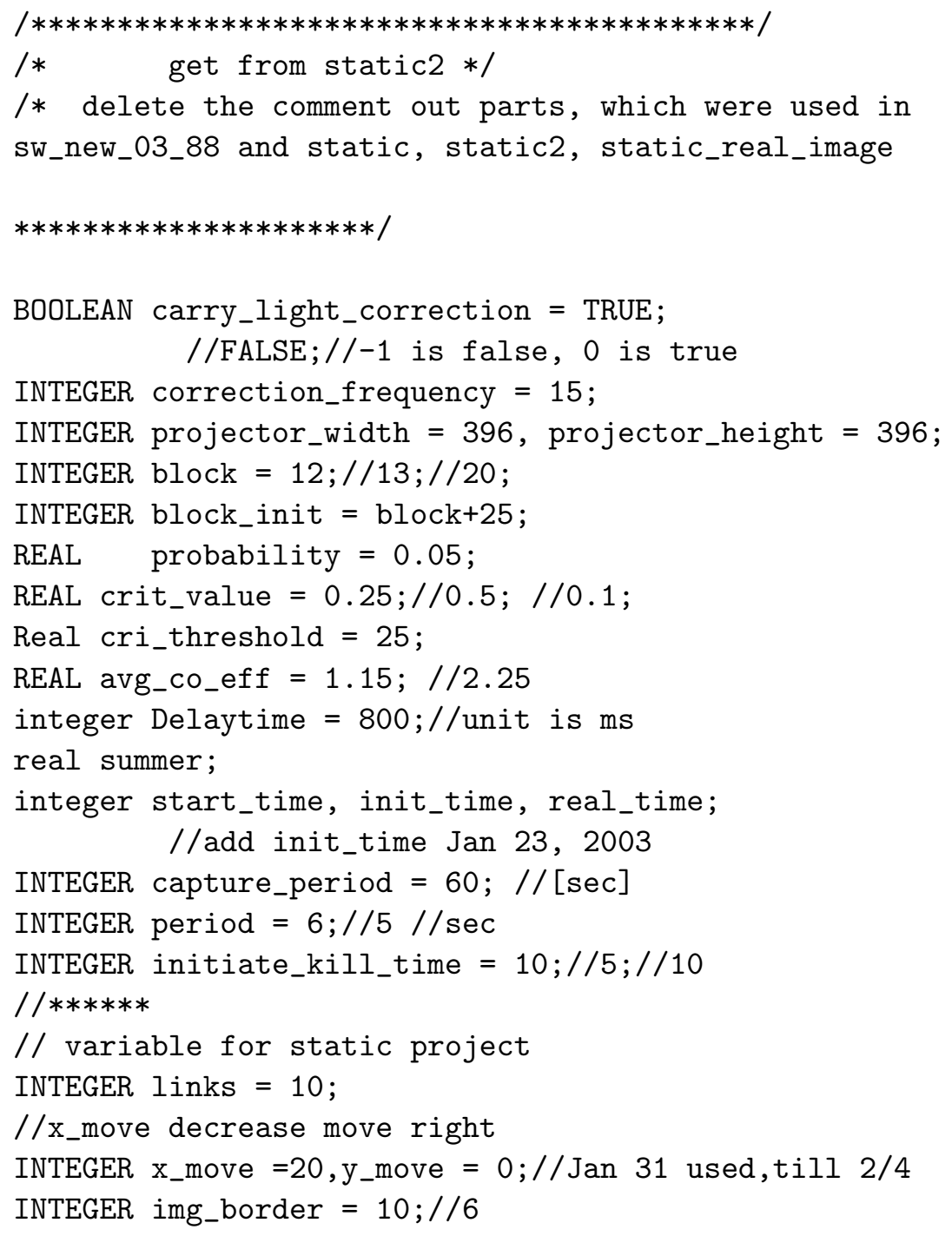




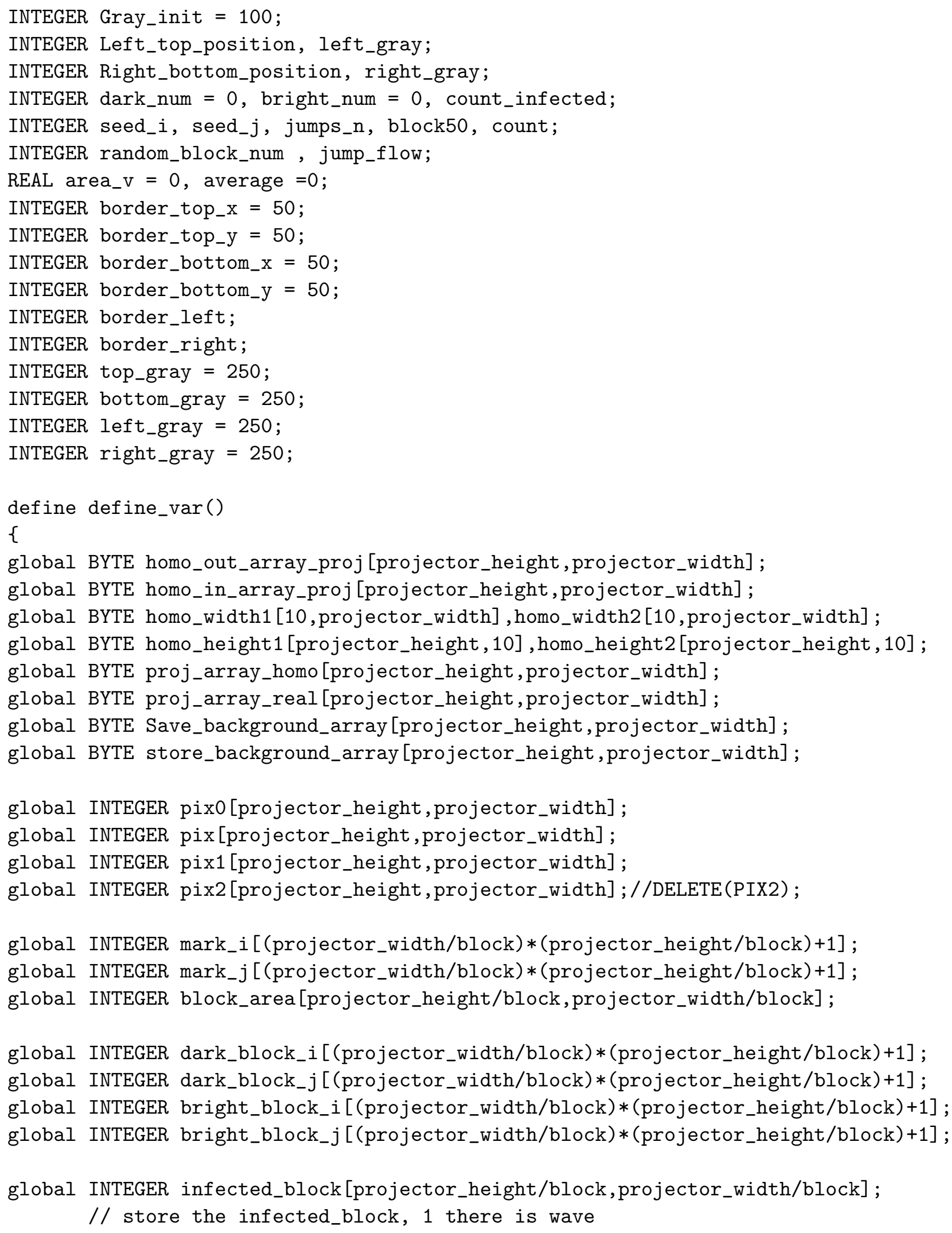


global INTEGER link_infected_block[projector_height/block,projector_width/block];

// check if there is a wave in the sources of links,

// it will be set to 0 after each checking in start function

global INTEGER life_time[projector_height/block,projector_width/block];

global INTEGER block_num; //delete(block_num);

global INTEGER mark_block_i[(projector_width/block)*(projector_height/block) +1$]$;

global INTEGER mark_block_j[(projector_width/block)*(projector_height/block)+1];

global INTEGER block_label[projector_height/block,projector_width/block,multiplicity]; global REAL test_gray[projector_height,projector_width];

global BYTE homo_array[projector_height,projector_width]=Gray_init;

global INTEGER src_x[LINKS], src_y[LINKS], dst_x[LINKS], dst_y[LINKS]; \}

define_var();

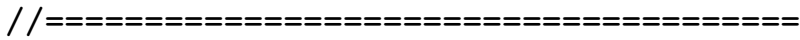

CHAR Image_file_name;

CHAR Image_file_name_bg;

CHAR Image_file_path="D:/Jianxia/SW/static/July/0722/0722-1/0722-1" ;

INTEGER

$\mathrm{xBrightness \_ range}=0: 255: 220$,

$\mathrm{xBrightness}=220$,

xContrast_range $=0: 255: 200, / / 170$

$\mathrm{xContrast}=200$;

INTEGER

xBrightness_range $2=0: 255: 210$,

$\mathrm{xBrightness} 2=210$,

xContrast_range $2=0: 255: 160$,

$\mathrm{xContrast2}=160$;

INTEGER Frame_counter $=0$;

INTEGER Frame_counter_save = 0 ;

CHAR xStatus = "Initializing";

INTEGER projector_middle;

INTEGER middle_xp, middle_yp;//show(middle_xp) ; show(proj_roi);

INTEGER camera_roi,camera_roi_2;

INTEGER proj_roi [4], proj_homo_roi [4];

REAL real_proj_homo_roi [4];

//open projector window (image)

NewImage ("SoftwareFixed", "Projector",

$796: 558$,

$0: 1: 8: 1: 1: 3$, Modellmage, ); 


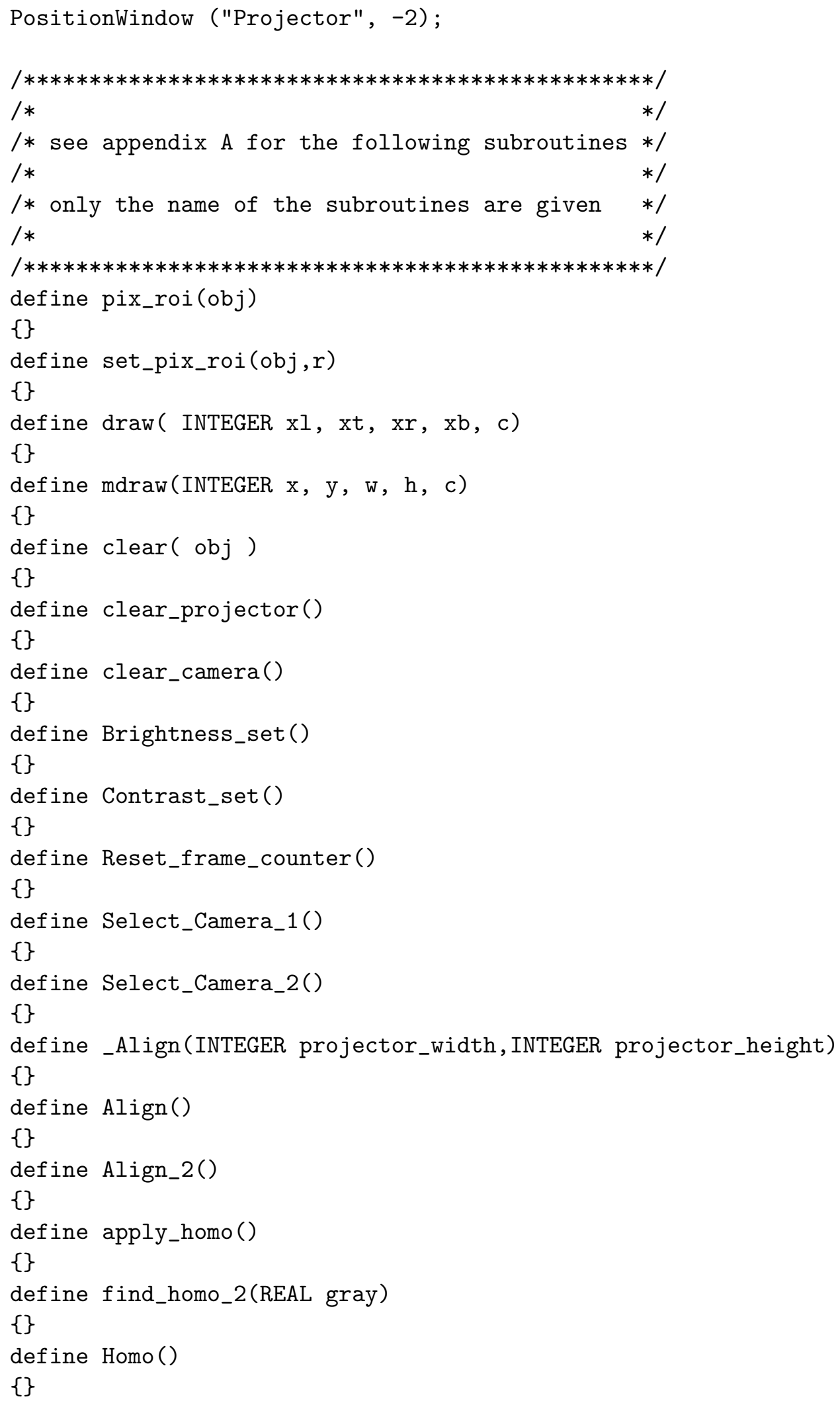




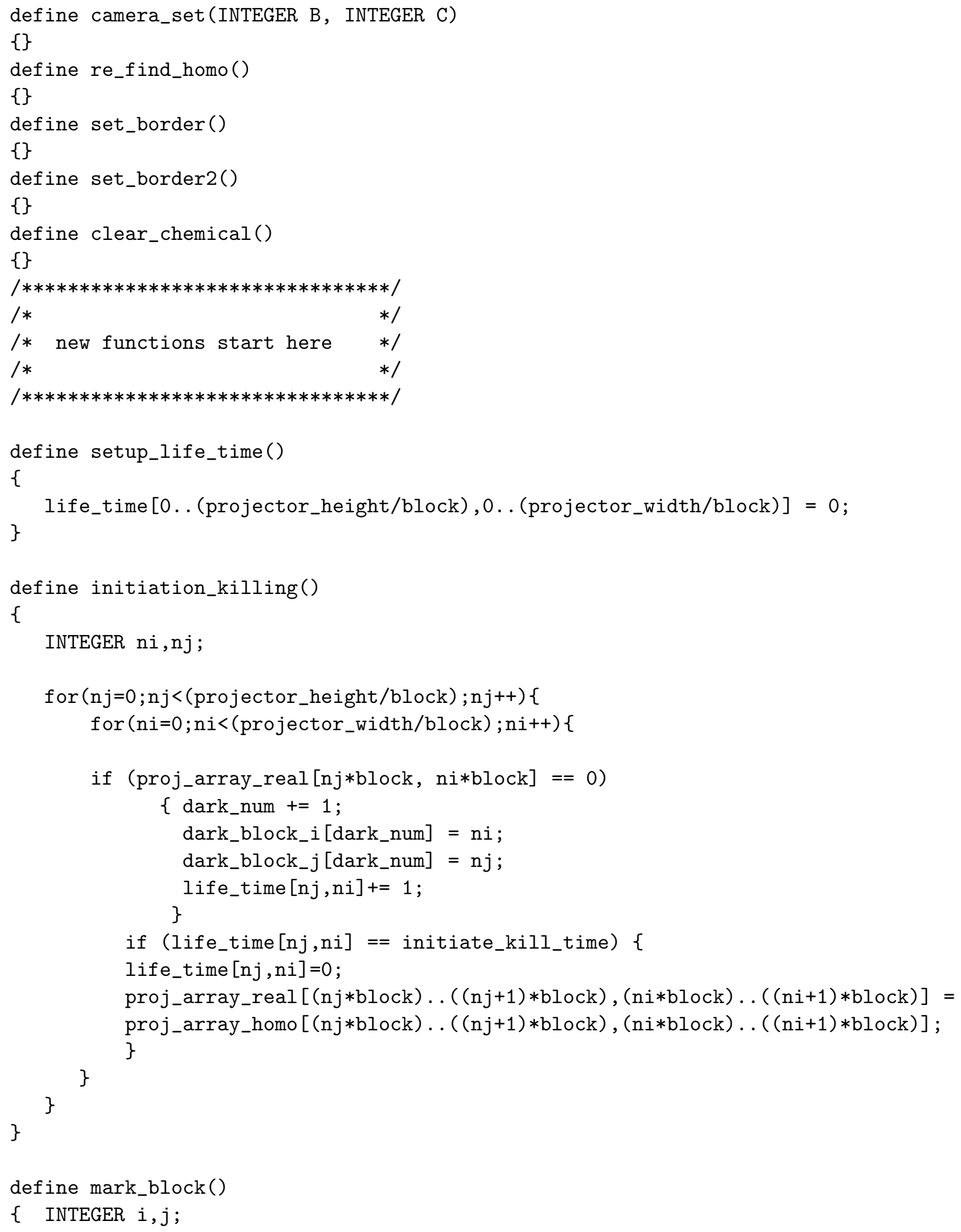




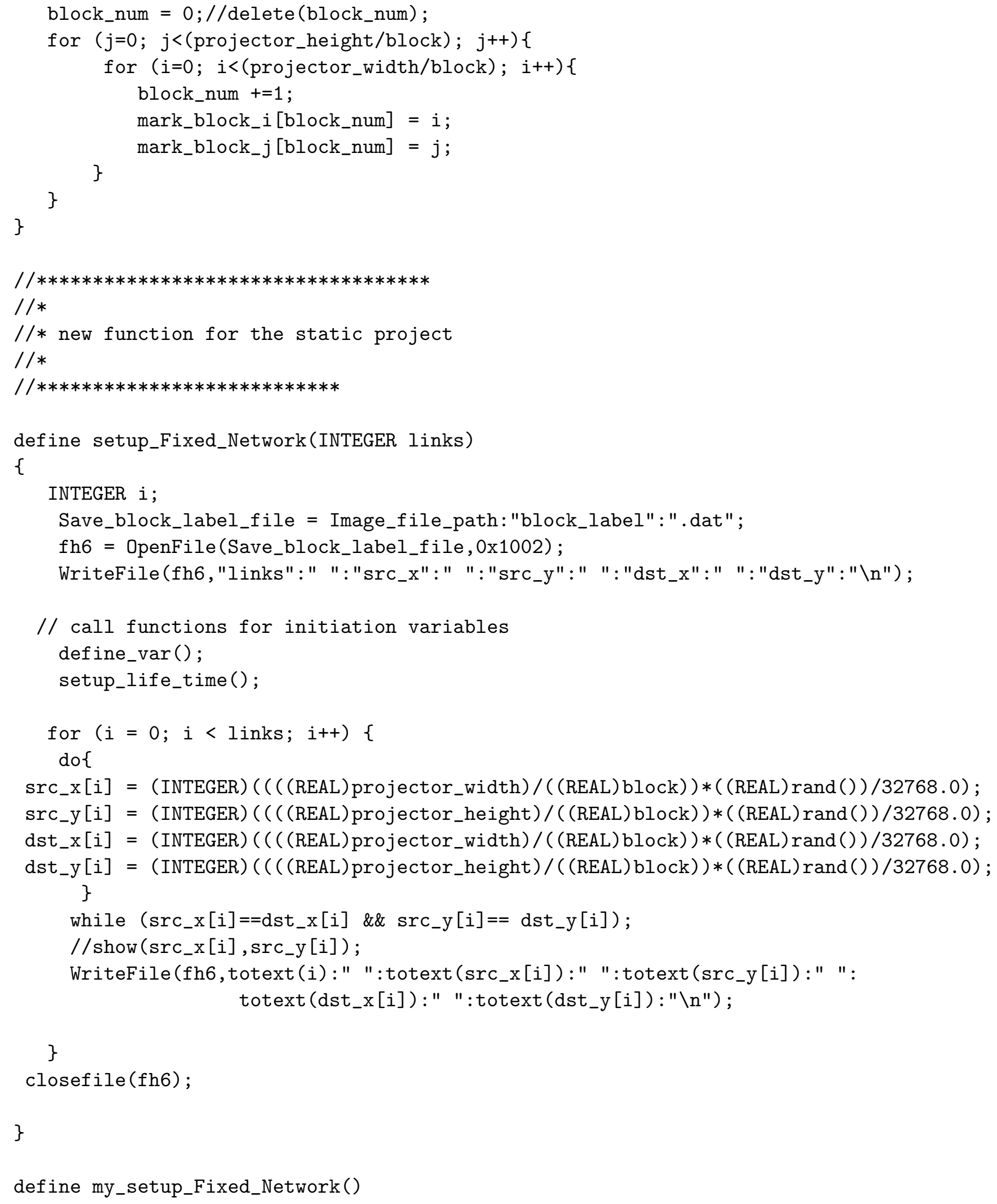




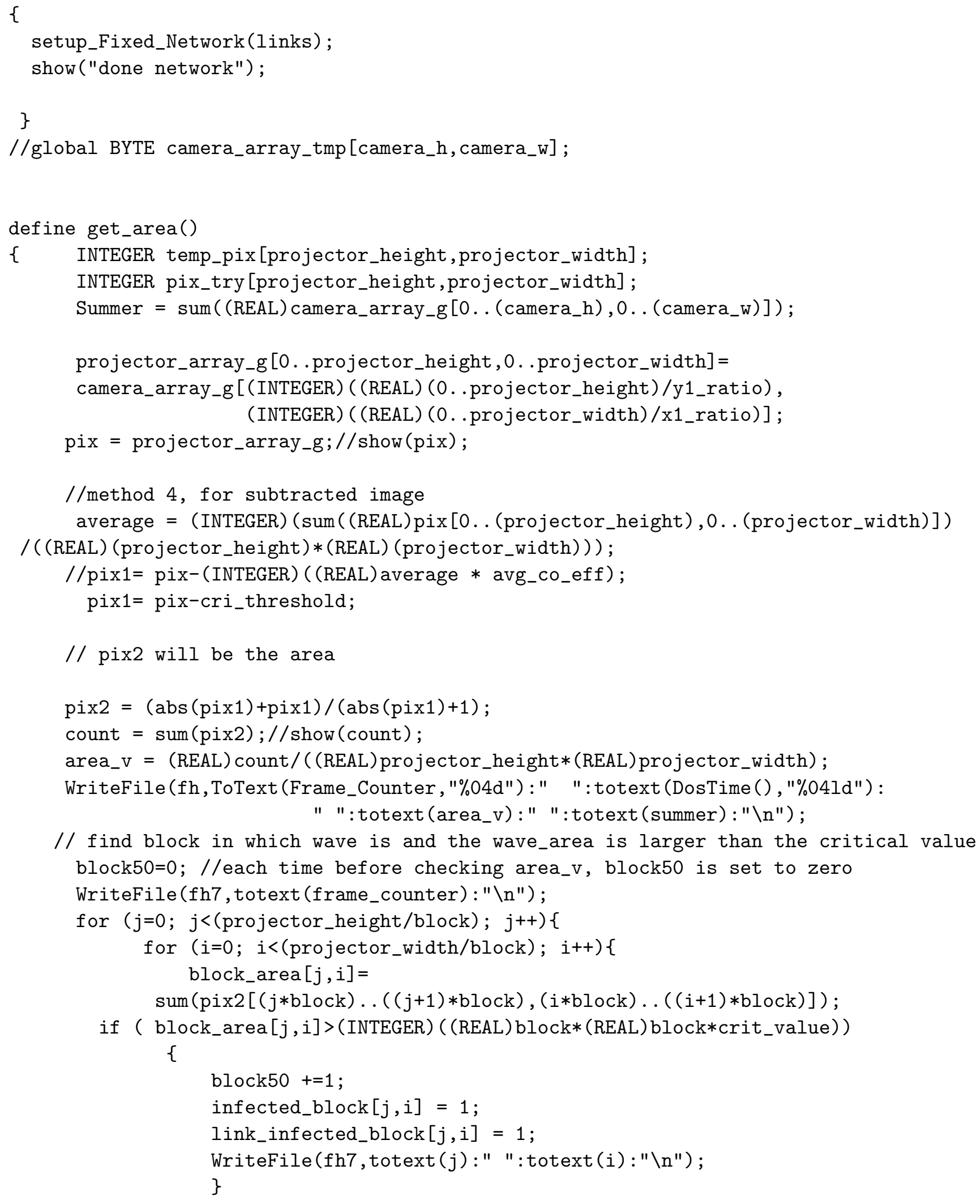




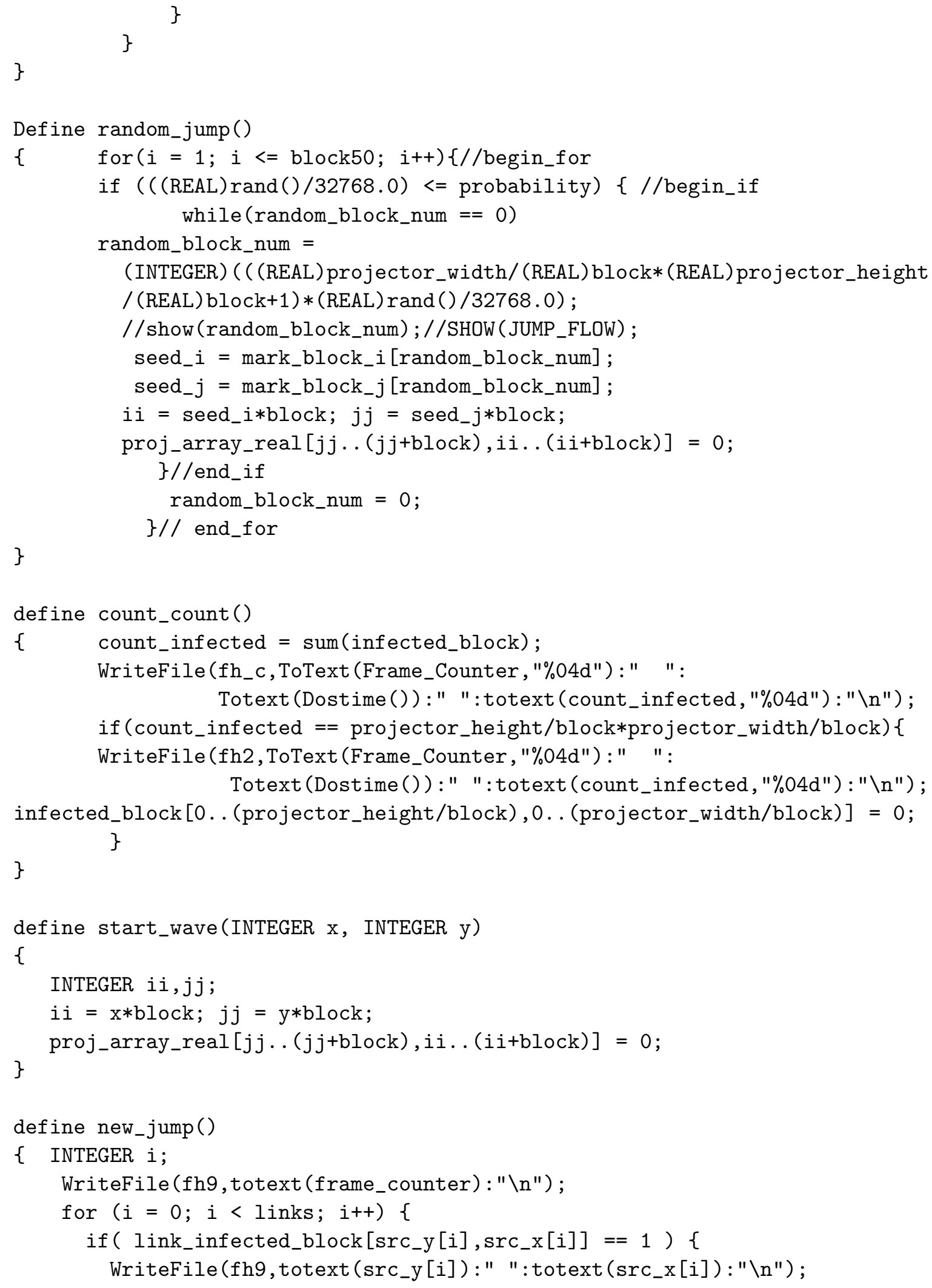




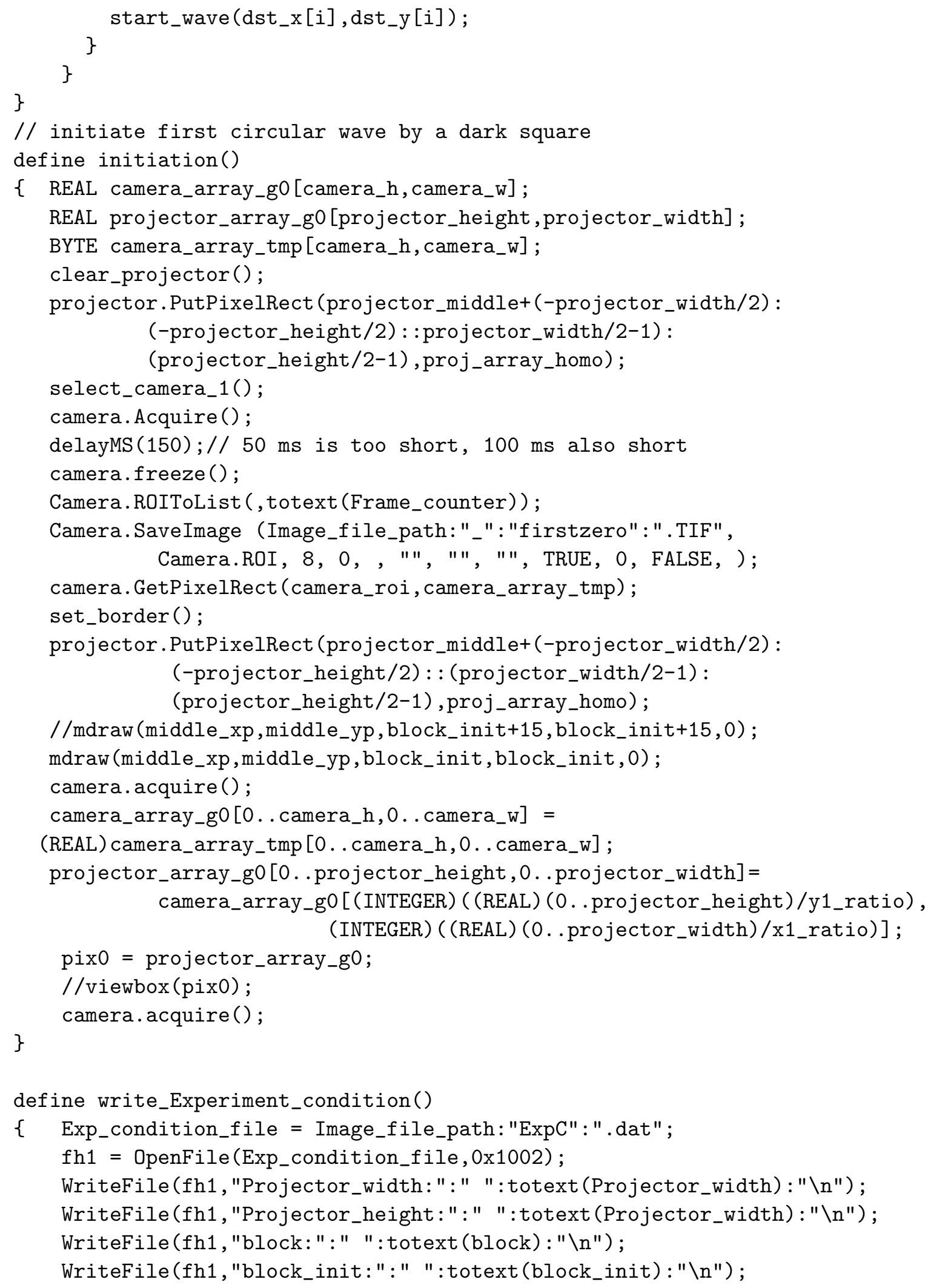




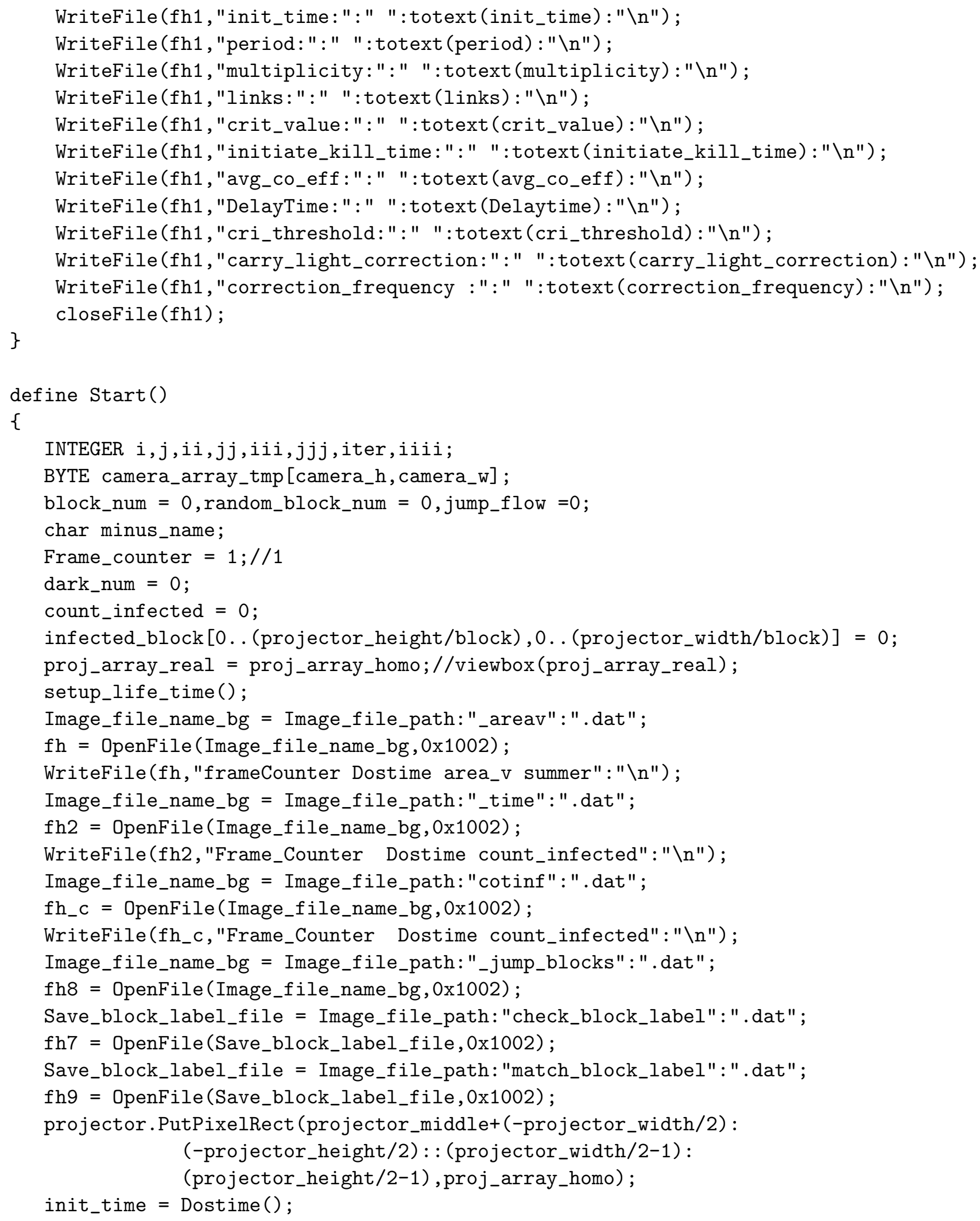




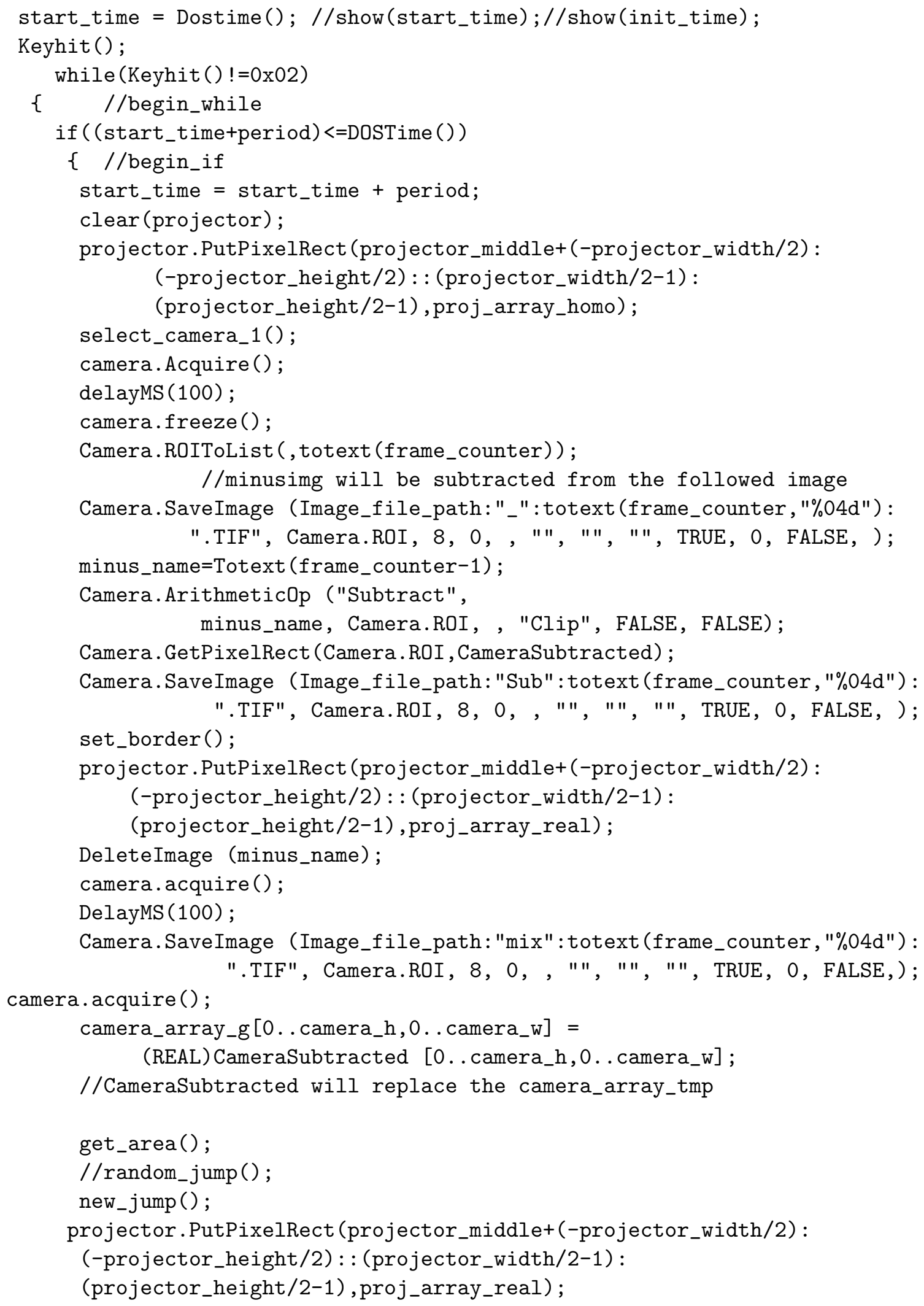




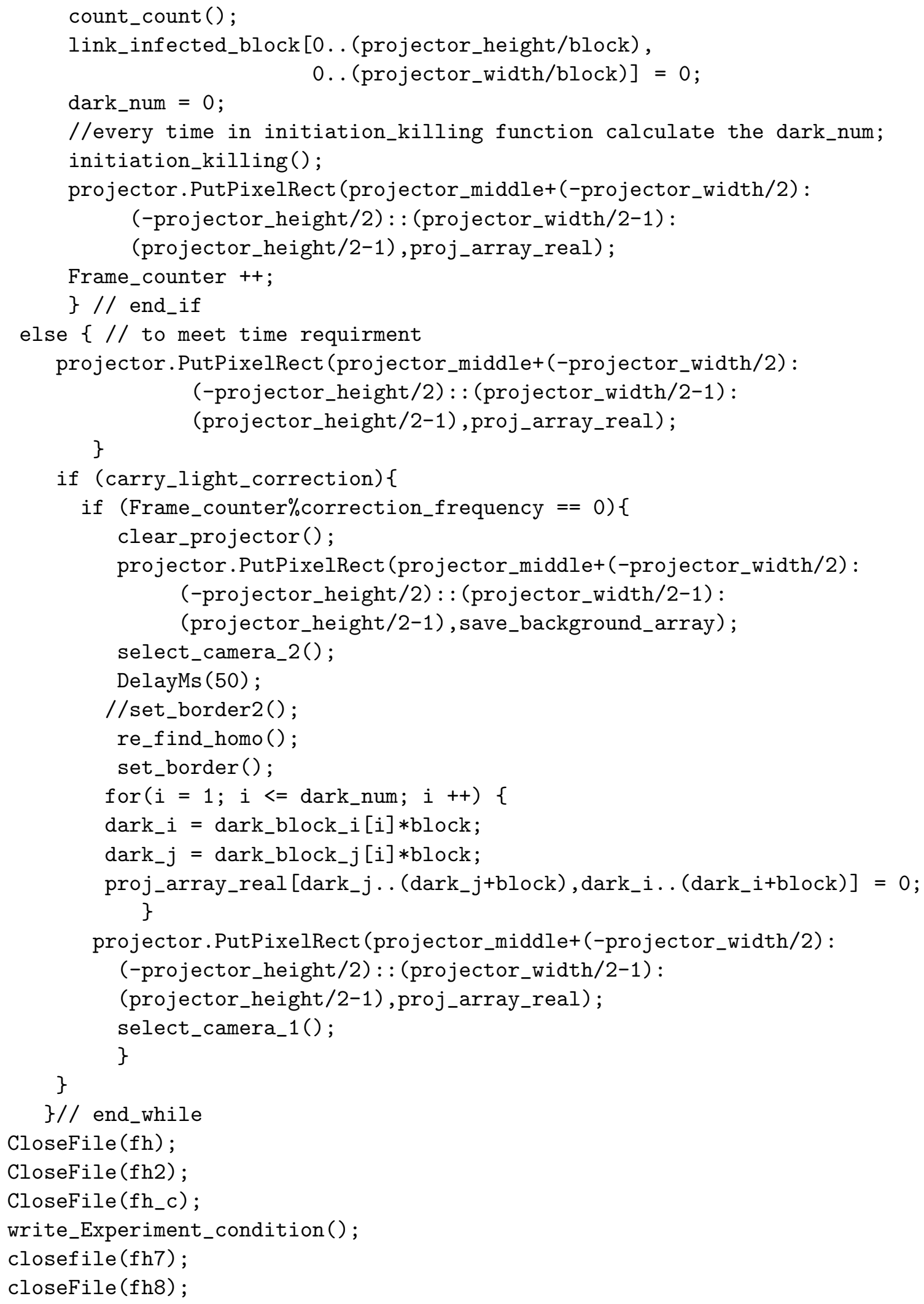




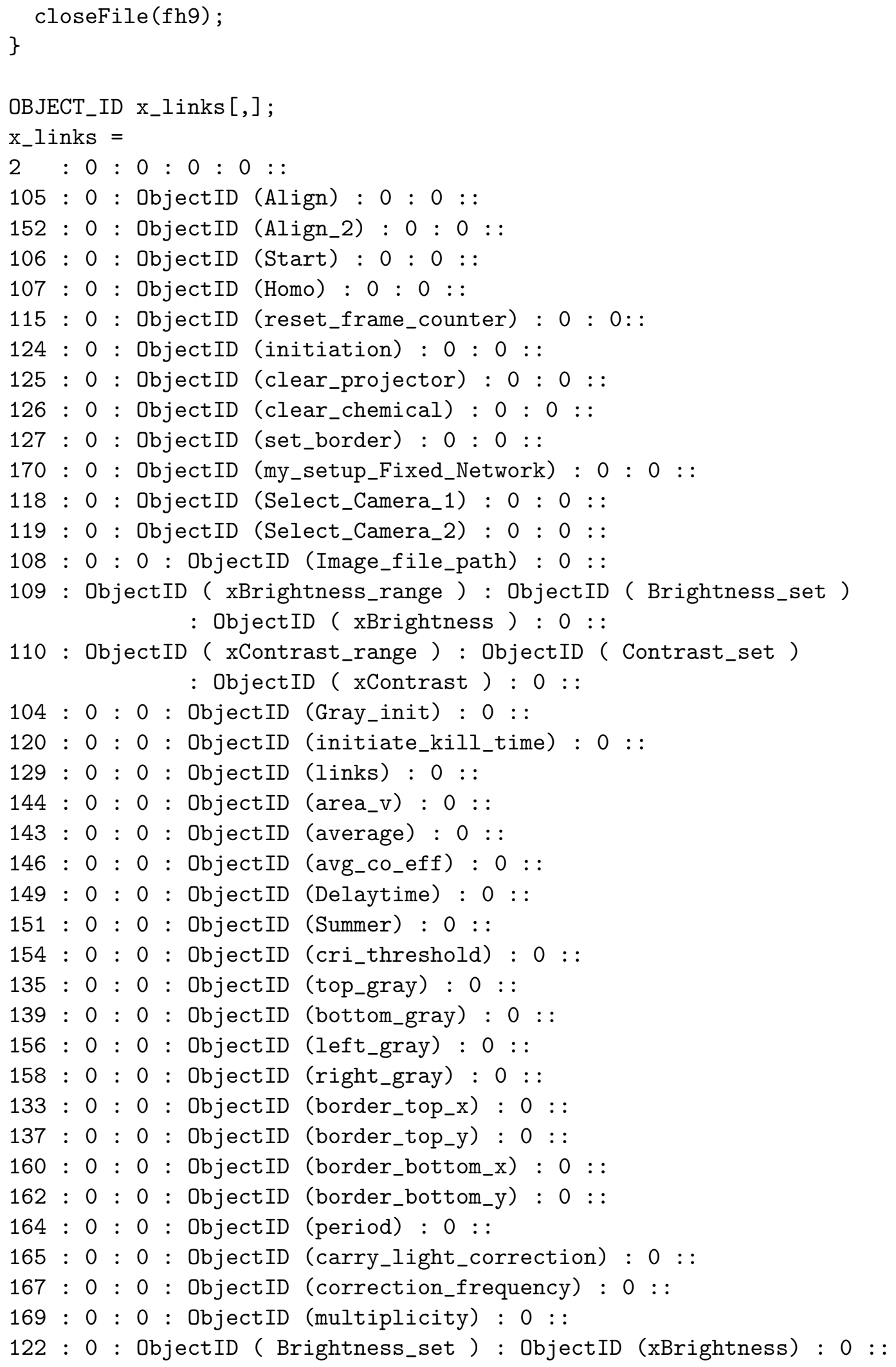




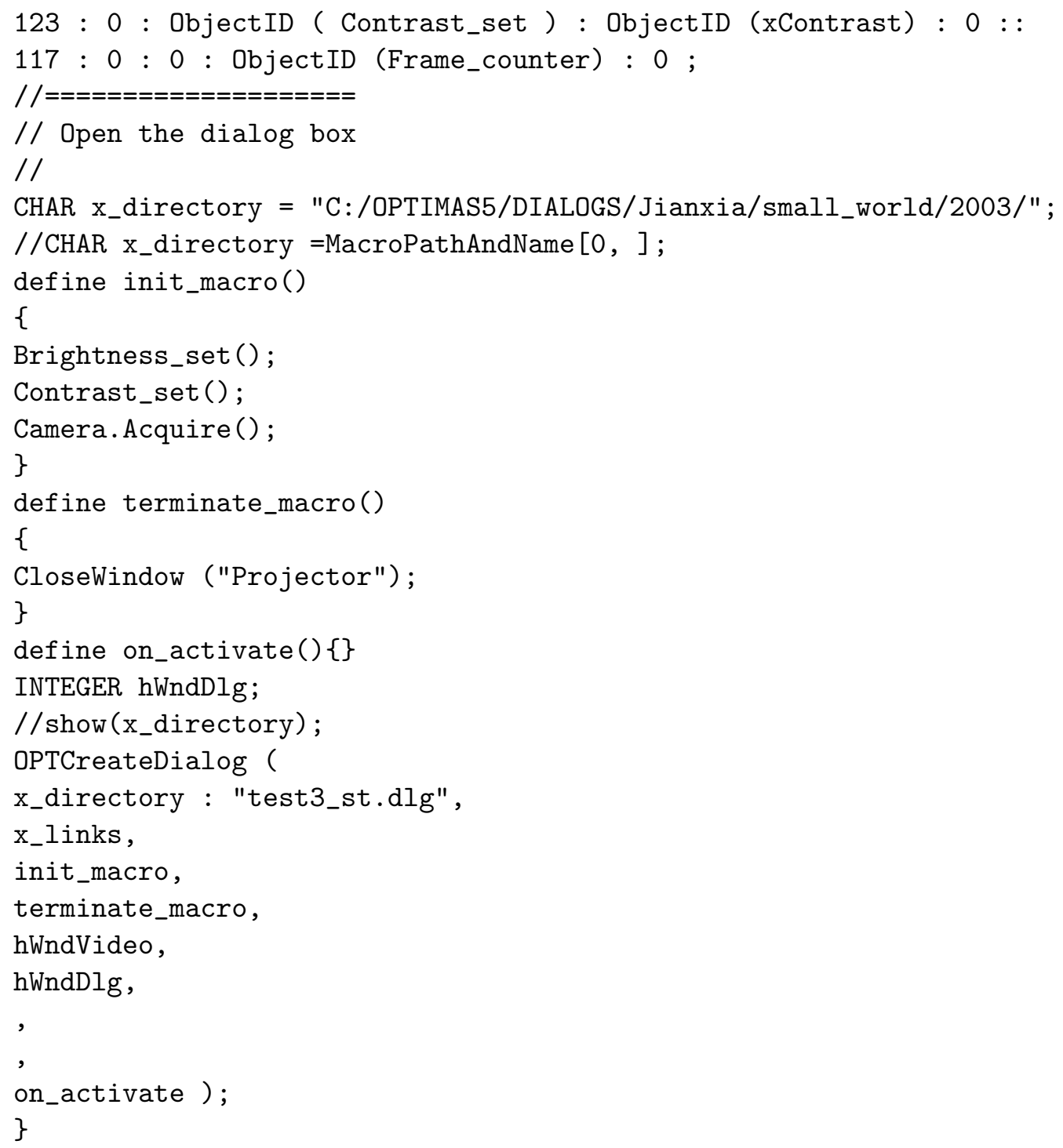




\subsection{References}

[1] Fred Brauer, "Basic Ideas of Mathematical Epidemiology," in Mathematical Approaches for Emerging and Reemerging Infectious Diseases: An Introduction (Springer-verlag, 2001).

[2] J. Graunt, "Natural and Political Observations Made Upon the Bills of Mortality," (John Martin, London, 1662).

[3] D. Bernouilli, "Essai D'une nouvelle analyse de la mortalitè casuèe par la petite vèrole et des avantages de l'inoculation pour la prèvenir," Mèm. Math. Phys. Acad. Roy. Sci., Pairs, 1-45 (1760).

[4] W. Farr, "Progress of Epidemics," In Second Annual Report of the RegistrarGeneral of Births, Deaths and Marriages in England, 91-98 (1840).

[5] D. J. Daley and J. Gani, Epidemic Modeling: An Introduction (Cambridge University Press, Cambridge, 1999).

[6] W. H. Hamer, "The Milroy Lectures on Epidemic Disease in England - The Evidence of Variability and Persistency of Type. Lecture III," Lancet 1, 733-739 (1906).

[7] R. Ross, "An Application of the Theory of Probabilities to the Study of A Priori Pathemetry I," Proc. Roy. Soc. Lond. A 92, 204-230 (1916).

[8] W. O. Kermack and A. G. McKendrick, "A Contribution to the Mathematical Theory of Epidemics," Proc. Royal Soc. London 115, 700-721 (1927).

[9] R. M. Anderson and R. M. May, Infectious Diseases in Humans: Dynamics and Control (Oxford University Press, Oxford, New York, 1991).

[10] N. T. J. Bailey, The Mathematical Theory of Infectious Diseases and its Applications (Hafner Press, New York, 1975). 
[11] C. J. Mode and C. K. Sleeman, Stochastic Processes in Epidemiology (World Scientific, Singapore, 2000).

[12] W. O. Kermack and A. G. McKendrick, "Contributions to the Mathematical Theory of Epidemics Part II," Proc. Royal Soc. London 138, 55-83 (1932).

[13] Fred Brauer, "Extensions of the Basic Models," in Mathematical Approaches for Emerging and Reemerging Infectious Diseases: An Introduction (Springer-Verlag, 2001).

[14] R. M. Anderson and R. M. May, "Population Biology of Infectious Diseases: Part I," Nature 280, 361-367 (1979).

[15] M Girvan, Duncan S. Callaway, M. E. J. Newman, and Steven H. Strogatz, "Simple Model of Epidemics with Pathogen Mutation," Phys. Rev. E 65, 0319151-9 (2002).

[16] http://www.who.int/csr/sars/en/

[17] W. H. McNeill, Plagues and Peoples (Anchor Press/Doubleday, Garden City, New York, 1976).

[18] J. D. Murray, Mathematical Biology (Springer, Berlin, 1993).

[19] N. T. J. Bailey, "Spatial Models in the Epidemiology of Infectious Diseases," in Biological Growth and Spread Vol. 38 of Lecture Notes in Biomathematics (H. Rost and P. Tautu ed.) (Springer-Verlag, 1980).

[20] E. B. Wilson and J. Worester, "The Spread of an Epidemic," Proc. Nat. Acad. Sci. 31, 327-332 (1945).

[21] L. A. Rvachev and I. M. Longini, "A Mathematical Model of the Global Spread of Influenza," Math. Biosci. 75, 3-22 (1985).

[22] I. M. Longini, "A Mathematical Model for Predicting the Geographic Spread of New Infectious Agents," Math. Biosci. 90, 367-383 (1988). 
[23] L. Sattenspiel and K. Dietz, "A Structured Epidemic Model Incorporating Geographic Mobility Among Regions," Math. Biosci. 128, 71-91 (1995).

[24] L. Sattenspiel and D. A. Herring, "Structured Epidemic Models and the Spread of Influenza in the Central Canadian Subarctic," Hum. Biol. 70, 91-115 (1998).

[25] L. Sattenspiel, A. Movarry, and D. A. Herring, "Modeling the Influence of Settlement Structure on the Spread of Influenza Among Communities," Am. J. Hum. Biol. 12, 736-748 (2000).

[26] J. Arino and P. van den Driessche, "A Multi-City Epidemic Model," Math. Pop. Studies, 10, 175-193 (2003).

[27] N. Boccara and K. Cheong, "Automata Network SIR Models for the Spread of Infectious Diseases in Populations of Moving Individuals," J. Phys. A 25, 24472462 (1992).

[28] N. Boccara and K. Cheong, "Critical Behaviour of a Probabilistic Automata Network SIS Model for the Spread of an Infectious Disease Population of Moving Individuals," J. Phys. A 26, 3707-3717 (1992).

[29] N. Boccara, K. Cheong, and M. Oram, "Probabilistic Automata Epidemic Model with Births and Deaths Exhibiting Cyclic Behavior," J. Phys. A 27, 1585-1597 (1994).

[30] O. Miramontes and B. Luque, "Dynamical Small-World Behavior in an Epidemical Model of Mobile Individuals," Physica D 168, 379-385 (2002).

[31] S. C. Manrubia, J. Delgado, and B. Luque, "Small-World Behaviour in a System of Mobile Elements," Europhys. Lett. 53, 693-700 (2001).

[32] S. N. Dorogovtsev and J. F. F. Mendes, "Evolution of Networks," Adv. Phys. 51, 1079-1187 (2002). 
[33] A.-L. Barabàsi and R. Albert, "Statistical Mechanics of Complex Networks," Rev. Mod. Phys. 74, 47-97 (2002).

[34] S. H. Strogatz, "Exploring Complex Networks," Nature 410, 268-277 (2001).

[35] R. Albert and A-L Barabàsi, "Statistical Mechanics of Complex Networks," Rev. Mod. Phys. 74, 47-97 (2002).

[36] D. J. Watts and S. H. Strogatz, "Collective Dynamics of 'Small-World' Networks," Nature 393, 440-442 (1998).

[37] D. J. Watts, "Small Worlds: the Dynamics of Networks Between Order and Randomness," (Princeton University Press, Princeton, New Jersey, 1999).

[38] R. Pastor-Satorras and A. Vespignani, "Epidemic Spreading in Scale-Free Networks," Phys. Rev. Lett. 86, 3200-3203 (2001).

[39] M. Kuperman and G. Abramson "Small World Effect in an Epidemiological Model," Phys. Rev. Lett. 86, 2909-2912 (2001).

[40] D. S. Callaway, M. E. J. Newman, S. H. Strogatz, and D. J. Watts, "Network Robustness and Fragility: Percolation on Random Graphs," Phys. Rev. Lett. 85, 5468-5472 (2000).

[41] M. E. Newman and D. J. Watts, "Renormalization Group Analysis of the SmallWorld Network Model," Phys. Lett. A 263, 341-347 (1999).

[42] C. Moore and M. E. J. Newman, "Epidemics and Percolation in Small-World Networks," Phys. Rev. E 61, 5678-5682 (2000).

[43] E. Ahmed, A. S. Hegazi, and A. S. Elgazzar, "An Epidemic Model on SmallWorld Networks and Ring Vaccination," Int. J. Mod. Phys. 13, 189-198 (2002).

[44] N. T. J. Bailey, "The Simulation of Stochastic Epidemics in Two Dimensions," Proc. Fifth Berkeley Symp. Math. Statist. \& Prob. 4, 237-257 (1967). 
[45] Y. Moreno, R. Pastor-Satorras, and A. Vespignani. "Epidemic Outbreaks in Complex Heterogeneous Networks," Eur. Phys. J. B 26, 521-529 (2002).

[46] C. P. Warren, L. M. Sander, and I. Sokolov, "Epidemics, Disorder, and Percolation," Physica A. 325, 1-8 (2003).

[47] L. M. Sander, C. P. Warren, I. M. Sokolov, C. Simon, and J. Koopman, "Percolation on Heterogeneous Networks as a Model for Epidemics," Math. Biosci. 180, 293-305 (2002).

[48] M. E. J. Newman, I. Jensen, and R. M. Ziff, "Percolation and Epidemics in a Two-Dimensional Small World," Phys. Rev. E 65, 021904-1-7 (2002).

[49] A. L. Lloyd and R. M. May, "How Viruses Spread Among Computers and People," Science 292, 1316-1317 (2001).

[50] M. E. J. Newman and D. J. Watts, "Scaling and Percolation in the Small-World Network Model," Phys. Rev. E 60, 7332-7342 (1999).

[51] D. Volchenkov, L. Volchenkova, and Ph. Blanchard, "Epidemic Spreading in a Variety of Scale Free Networks," Phys. Rev. E 66, 046137-1-9 (2001).

[52] R. Pastor-Satorras and A. Vespignani, "Epidemic Dynamics and Endemic States in Complex Networks," Phys. Rev. E 63, 066117-1-8 (2001).

[53] R. M. May and A. L. Lloyd, "Infection Dynamics on Scale-Free Networks," Phys. Rev. E 64, 066112-1-4 (2001).

[54] Y. Moreno and A. Vàzquez "Disease Spreading in Structured Scale-Free Networks," Eur. Phys. J. B 31, 265-271 (2003).

[55] M. Boguña, R. Pastor-Satorras, and A. Vespignani, "Absence of Epidemic Threshold in Scale-Free Networks with Degree Correlations," Phys. Rev. Lett. 90, 028701-1-4 (2003). 
[56] M. Boguña, R. Pastor-Satorras, and A. Vespignani, "Epidemic Spreading in Correlated Complex Networks," Phys. Rev. E 66, 047104-1-4 (2002).

[57] M. E. J. Newman, "Spread of Epidemic Disease on Networks," Phys. Rev. E 66, 016128-1-11 (2002).

[58] M. Boguña, R. Pastor-Satorras, and A. Vespignani, "Epidemic Spreading in Complex Networks with Degree Correlations," in Statistical Mechanics of Complex Networks (Springer, Berlin, New York, 2003).

[59] R. M. May and A. L. Lloyd, "Infection Dynamics on Scale-Free Networks," Phys. Rev. E 64, 0166112-1-4 (2001).

[60] S. Milgram, "The Small World Problem," Psychol. Today 2, 60-67 (1967).

[61] M. Kochen, ed. The Small World (Norwood, Ablex, 1989).

[62] J. Guare, Six Degrees of Separation: A Play (New York: Vintage, 1990).

[63] A.-L. Barabasi and R. Albert, "Emergence of Scaling in Random Networks," Science 286, 509-512 (1999).

[64] D. Volchenkov and Ph. Blanchard, "An Algorithm Generating Random Graphs with Power Law Degree Distributions," Physica A 315, 677-691 (2002).

[65] K. Klemm and V. M. Eguíluz, "Highly Clustered Scale-Free Networks," Phys. Rev. E 65, 036123-1-5 (2002).

[66] M. Faloutsos, P. Faloutsos, and C. Faloutsos, "On Power-Law Relationships of the Internet Topology," Comput. Commun. Rev. 29, 251-263 (1999).

[67] G. Caldarelli, R. Marchetti, and L. Pietronero, "The Fractal Properties of Internet," Europhys. Lett. 52, 386-392 (2000).

[68] R. Albert, H. Jeong, and A.-L. Barabasi, "Internet: Diameter of the World-Wide Web," Nature 401, 130-131 (1999). 
[69] F. Liljeros, C. R. Edling, L. A. N. Amaral, H. E. Stanley, and Y. Åberg, "The Web of Human Sexual Contacts," Nature (London) 411, 907-908 (2001).

[70] R. J. Field and R. M. Noyes, "Oscillations in Chemical Systems. IV. Limit Cycle Behavior in a Model of a Real Chemical Reaction," J. Chem. Phys. 60, 1877-1884 (1974).

[71] J. J. Tyson and P. C. Fife, "Target Patterns in a Realistic Model of the BelousovZhabotinskii Reaction," J. Chem. Phys. 73, 2224-2237 (1980).

[72] H. J. Krug, L. Pohlmann, and L. Kuhnert, "Analysis of the Modified Complete Oregonator Accounting for Oxygen Sensitivity and Photosensitivity of BelousovZhabotinsky Systems," J. Phys. Chem. 94, 4862-4866 (1990).

[73] S. Kádár, T. Amemiya, and K. Showalter, "Reaction Mechanism for Light Sensitivity of the $\mathrm{Ru}$ (bpy) ${ }_{3}{ }^{2+}$-Catalyzed Belousov-Zhabotinsky Reaction," J. Phys. Chem. A 101, 8200-8206 (1997).

[74] J. Wang, S. Kádár, P. Jung, and K. Showalter, "Noise Driven Avalanche Behavior in Subexcitable Media," Phys. Rev. Lett. 82, 855-858 (1999).

[75] http://linkage.rockefeller.edu/wli/zipf/

[76] http://www.few.eur.nl/few/people/vanmarrewijk/geography/zipf/

[77] L. Sattenspiel and C. Powell, "Geographic Spread of Measles on the Island of Dominica, West Indies," Hum. Biol. 65, 107-129 (1993).

[78] A. Cliff and P. Haggett, "Island Epidemics," Sci. Am. 250, 138-147 (1984).

[79] P. Rohani, D. J. D. Earn, and B. T. Grenfell, "Opposite Patterns of Synchrony in Sympatric Disease Metapopulations," Science 286, 968-971 (1999). 